

\section{EDITORIAL BOARD}

ANTONIO CARCATERRA ERIC A. CARLEN

FRANCESCO DELL'ISOLA

RAFFAELE ESPOSITO

ALBERT FANNJIANG

Gilles A. FrancFort

PiERANGElo MARCATI

JEAN-JACQUES MARIGO

PETER A. MARKOWICH MARTIN OSTOJA-STARZEWSKI

PIERRE SEPPECHER

DAVID J. STEIGMANN

PAUl STEINMANN

PierRe M. Suquet

\section{MANAGING EDITORS}

MICOL AMAR

CORRADO LATTANZIO

ANGELA MADEO

MARTIN OSTOJA-STARZEWSKI

\section{ADVISORY BOARD}

ADNAN AKAY

Holm AltenbaCH

MICOL AMAR

HARM ASKES

TEODOR ATANACKOVIĆ

VICTOR BERDICHEVSKY

GuY BouchitTÉ

ANDREA BRAIDES

ROBERTO CAMASSA

MAURO CARFORE

ERIC DARVE

FELIX DARVE

ANNA DE MASI

Gianpietro Del Piero

EMMANUELE Di BENEDETTO

BERNOLD FIEDLER

IRENE M. GAMBA

SERGEY GAVRILYUK

TIMOTHY J. HEALEY

DOMINIQUE JEULIN

ROGER E. KHAYAT

CORRADO LATTANZIO

ROBERT P. LIPTON

ANGELO LUONGO

ANGEla MadeO

JUAN J. MANFREDI

CARLO MARCHIORO

GÉrard A. MAUGin

ROBERTO NATALINI

PATRIZIO NEFF

ANDREY PIATNITSKI

ERrico Presutti

MARIO PUlVIRENTI

LuCiO RuSSO

Miguel A. F. SANJUAN

Patrick SElvadurai

ALEXANDER P. SEYRANIAN

MIROSLAV ŠILHAVÝ

GUIDO SWEERS

ANTOINETTE TORDESILLAS

LEV TRUSKINOVSKY

JUAN J. L. VELÁZQUEZ

VINCENZO VESPRI

ANGELO VULPIANI msp.org/memocs

Università di Roma “La Sapienza”, Italia

Rutgers University, USA

(CO-CHAIR) Università di Roma "La Sapienza", Italia

(TREASURER) Università dell'Aquila, Italia

University of California at Davis, USA

(CO-CHAIR) Université Paris-Nord, France

Università dell' Aquila, Italy

École Polytechnique, France

DAMTP Cambridge, UK, and University of Vienna, Austria

(CHAIR MANAGING EDITOR) Univ. of Illinois at Urbana-Champaign, USA

Université du Sud Toulon-Var, France

University of California at Berkeley, USA

Universität Erlangen-Nürnberg, Germany

LMA CNRS Marseille, France

Università di Roma “La Sapienza”, Italia

Università dell' Aquila, Italy

Université de Lyon-INSA (Institut National des Sciences Appliquées), France (CHAIR MANAGING EDITOR) Univ. of Illinois at Urbana-Champaign, USA

Carnegie Mellon University, USA, and Bilkent University, Turkey

Otto-von-Guericke-Universität Magdeburg, Germany

Università di Roma "La Sapienza”, Italia

University of Sheffield, UK

University of Novi Sad, Serbia

Wayne State University, USA

Université du Sud Toulon-Var, France

Università di Roma Tor Vergata, Italia

University of North Carolina at Chapel Hill, USA

Università di Pavia, Italia

Stanford University, USA

Institut Polytechnique de Grenoble, France

Università dell'Aquila, Italia

Università di Ferrara and International Research Center MEMOCS, Italia

Vanderbilt University, USA

Freie Universität Berlin, Germany

University of Texas at Austin, USA

Université Aix-Marseille, France

Cornell University, USA

École des Mines, France

University of Western Ontario, Canada

Università dell' Aquila, Italy

Louisiana State University, USA

Università dell' Aquila, Italia

Université de Lyon-INSA (Institut National des Sciences Appliquées), France

University of Pittsburgh, USA

Università di Roma "La Sapienza", Italia

Université Paris VI, France

Istituto per le Applicazioni del Calcolo "M. Picone", Italy

Universität Duisburg-Essen, Germany

Narvik University College, Norway, Russia

Università di Roma Tor Vergata, Italy

Università di Roma "La Sapienza", Italia

Università di Roma “Tor Vergata", Italia

Universidad Rey Juan Carlos, Madrid, Spain

McGill University, Canada

Moscow State Lomonosov University, Russia

Academy of Sciences of the Czech Republic

Universität zu Köln, Germany

University of Melbourne, Australia

École Polytechnique, France

Bonn University, Germany

Università di Firenze, Italia

Università di Roma La Sapienza, Italia

MEMOCS (ISSN 2325-3444 electronic, 2326-7186 printed) is a journal of the International Research Center for the Mathematics and Mechanics of Complex Systems at the Università dell'Aquila, Italy.

Cover image: "Tangle" by @ John Horigan; produced using the Context Free program (contextfreeart.org).

\section{PUBLISHED BY}

mathematical sciences publishers

nonprofit scientific publishing

http://msp.org/

(C) 2014 Mathematical Sciences Publishers 


\title{
A MIXED BOUNDARY VALUE PROBLEM IN POTENTIAL THEORY FOR A BIMATERIAL POROUS REGION: AN APPLICATION IN THE ENVIRONMENTAL GEOSCIENCES
}

\author{
A. P. S. SElvadurai
}

\begin{abstract}
This paper develops an exact closed-form solution to a mixed boundary value problem in potential theory for an elliptical opening located at an impervious interface separating two dissimilar, nondeformable porous media. The resulting solution provides a convenient result for estimating the leakage rate of an incompressible fluid retained in the system at a hydraulic potential difference. The result for the elliptical opening is also used to provide a Pólya-Szegö-type estimate for leakage rates from openings of arbitrary shape located at the impermeable interface. The extension of the study to include leakage into a transversely isotropic porous medium with the plane of isotropy inclined to the impervious boundary is also discussed.
\end{abstract}

\section{Introduction}

In environmental geosciences there are a number of instances where porous media are separated by relatively impervious barriers. Such situations can be found where periodic geologic deposition results in laminated regions where a fluid transmissivity contrast can be created by sedimentation of fine-grained material that will form a nearly impervious barrier. Examples of these include varved clays and other stratified geological media, where the scale of the impervious layers can range from a few millimeters to meters [Tschebotarioff 1951; Bear and Verruijt 1987; Phillips 1991; Selvadurai and Carnaffan 1997; Selvadurai et al. 2005]. An example that has direct relevance to the application discussed in this paper is the problem of geosynthetic liners, made of polymeric materials and used quite extensively as engineered barriers to prevent the migration of fluids containing hazardous and toxic chemicals from reaching potable groundwater regimes. Nearly all current-day waste management endeavors, ranging from sanitary landfills to storage reservoirs from resource extraction, use a variation of the concept of a geosynthetic liner to

\section{Communicated by Felix Darve.}

MSC2010: primary 31A10; secondary 31A25.

Keywords: potential theory, mixed boundary value problem, bimaterial region, Darcy flow, leakage from barrier, bounds for leakage rates, transverse isotropic permeability, mathematical geosciences. 
contain the hazardous components of toxic fluids. The implicit assumption in these endeavors is that the polymeric material will maintain its integrity as a barrier, in perpetuity. This is a naive expectation for any man-made material, particularly a geosynthetic material that is prone to degradation upon exposure to leachates and other chemicals and ultraviolet light. Recent experimental and theoretical investigations [Yu and Selvadurai 2005; 2007; Selvadurai and Yu 2006a; 2006b] indicate that the flexible polymeric material can be rendered brittle as a result of the loss of plasticizer, induced by the leaching action of relatively common chemicals such as acetone and ethanol. Since the plasticizer contributes to the flexibility of the geosynthetic material, the leaching process can lead to its embrittlement, which can serve as a location for the development of cracks through which the retained contaminant fluids can be released. The assessment of the leakage rate from the retained fluid is therefore of interest to estimate the environmental hazard associated with the long term use of geosynthetics.

This paper examines the problem of the leakage through an elliptical opening or defect that is located at the impermeable interface between two isotropic porous geological media (Figure 1). The formulation of the problem takes into consideration the Darcy flow properties of the porous media adjacent to the impervious barrier containing the elliptical defect. The paper examines the mixed boundary value in potential theory that can be applied to the fluid migration from an elliptical defect at an impervious boundary. It is shown that an exact solution to this problem can be obtained using the results originally presented by Lamb [1927] and recently applied by Selvadurai [2010], who developed a result for the intake shape factor for a circular fluid intake terminating at the interface between a hydraulically transversely isotropic porous medium and an impervious stratum with the principal planes of permeability inclined to the impervious interface. This analytical approach is used to develop an exact analytical result for the leakage from an elliptical defect in an otherwise impervious geosynthetic liner. The analytical procedure yields an exact closed-form solution, which takes into consideration the permeability characteristics of porous regions on either side of the impervious barrier. It is shown that the analytical result for the elliptical defect can also be used to develop a set of bounds for estimating the leakage rate from a defect of arbitrary shape. In the particular instance when the barrier with the elliptical defect is in contact with a single transversely isotropic porous medium with the plane of transverse isotropy inclined to the impervious interface, a closed-form analytical result can be developed for the leakage rate from the elliptical defect.

\section{The mixed boundary value problem}

We restrict attention to a bimaterial porous region that contains a plane impermeable barrier at the interface of the two porous media and fluid leakage occurs 


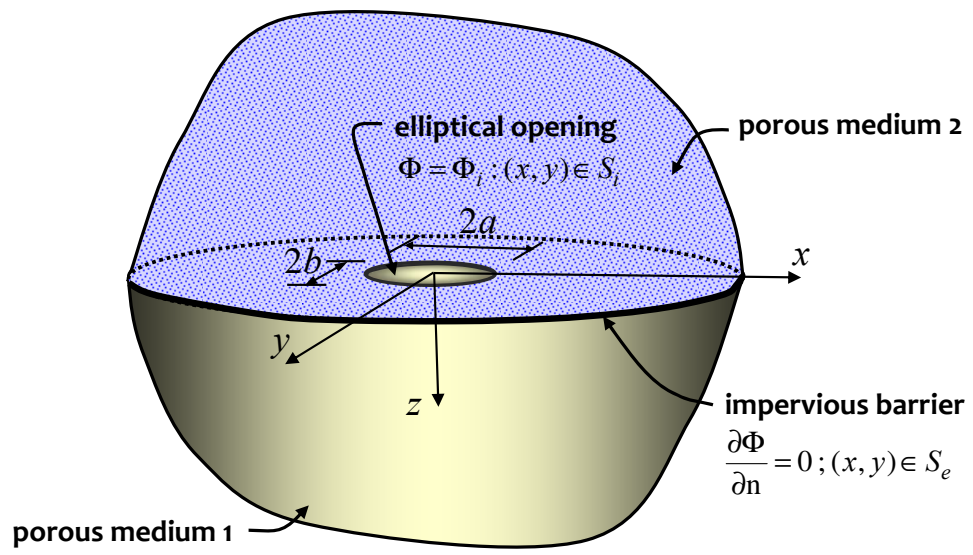

Figure 1. Elliptical opening at an impermeable interface separating two dissimilar porous media.

through an elliptical defect in the impervious barrier (Figure 1). The fluid flow characteristics are governed by Darcy's law and the permeabilities are defined by $K_{1}$ and $K_{2}$ for the respective regions. The reduced Bernoulli potential associated with Darcy flow is defined by $\Phi(\boldsymbol{x})$ and this neglects the velocity potential. The datum is taken as the plane of the interface and we assume that the regions 1 and 2 are subjected, respectively, to far-field reduced Bernoulli potentials $\Phi_{1}$ and $\Phi_{2}$, with $\Phi_{1}>\Phi_{2}$. For purposes of model development, we assume that the nondeformable dissimilar porous region shown in Figure 1 contains fluids with similar properties, although it should be noted that the viscosity and other properties of the contaminating leachates can be different from those of groundwater. In order to develop a convenient analytical result that can be used to estimate the steady fluid leakage through the crack, we shall adopt this assumption. It can be shown that for isochoric Darcy flow in an isotropic nondeformable porous medium the mass conservation law gives $\nabla \cdot \boldsymbol{v}=0$, where $\boldsymbol{v}(\boldsymbol{x})$ is the velocity vector and $\boldsymbol{x}$ is the spatial coordinates. For an isotropic porous medium, Darcy's law can be written as

$$
\boldsymbol{v}(\boldsymbol{x})=-\frac{K \gamma_{w}}{\mu} \nabla \Phi,
$$

where $K$ is the permeability, $\gamma_{w}$ is the unit weight of the fluid, and $\mu$ is its dynamic viscosity. Combining Darcy's law and the fluid mass conservation principle, we obtain the partial differential equation governing $\Phi(\boldsymbol{x})$ as

$$
\nabla^{2} \Phi(\boldsymbol{x})=0,
$$

where $\nabla^{2}$ is Laplace's operator. We consider the mixed boundary value problem in potential theory referred to a halfspace region, the boundary of which is subject 
to the relevant Dirichlet and Neumann boundary conditions applicable to an impermeable region with an elliptical opening. The planar region $S_{i}$ corresponding to the elliptical defect is defined by

$$
S_{i}:\left(\frac{x}{a}\right)^{2}+\left(\frac{y}{b}\right)^{2} \leq 1,
$$

while the region $S_{e}$ exterior to the defect is defined by

$$
S_{e}:\left(\frac{x}{a}\right)^{2}+\left(\frac{y}{b}\right)^{2}>1,
$$

where $a$ and $b$ are, respectively, the semimajor and the semiminor axes of the elliptical region. We consider the mixed boundary value problem in potential theory referred to the elliptical aperture such that

$$
(\Phi)_{z=0}=\Phi_{i}=\mathrm{constant}, \quad(x, y) \in S_{i},
$$

and

$$
\left(\frac{\partial \Phi}{\partial z}\right)_{z=0}=0, \quad(x, y) \in S_{e},
$$

where $\Phi_{i}$ is the constant Bernoulli potential over the elliptical interior region, which is dictated by the constant pressure potential over this region, and the datum is taken as the plane of the defect. Since the problem examined has a three-dimensional configuration, the regularity conditions applicable to a semiinfinite domain should also be satisfied. In this case the far-field potential in region 1 is $\Phi_{1}$. For the solution of the mixed boundary value problem posed by (4) and (5) we assume, however, that the potential $\Phi(\boldsymbol{x})$ decays uniformly to zero as $\boldsymbol{x} \rightarrow \infty$. Since the Bernoulli potential is indeterminate to an arbitrary constant, the far-field value can be added to satisfy the value of the constant potential regularity condition as $x \rightarrow \infty$. The solution to the mixed boundary value problem in potential theory, governed by the partial differential equation (2) and mixed boundary conditions (4) and (5), can be developed in a variety of ways, the most widely accepted being the formulation that employs a generalized ellipsoidal coordinate system and by developing the solution to the opening with an elliptical plan form as a limiting case of an ellipsoid. This approach was used by Lamb [1927] to develop a solution for the motion of a perfect fluid through an elliptical aperture. The result can also be developed using the formal developments in potential theory given by Morse and Feshbach [1953, Section 10.3]. Similar developments have been used in [Green and Sneddon 1950; Kassir and Sih 1968; Selvadurai 1982; Walpole 1991] in developing canonical results for elliptical cracks and elliptical inclusions embedded in isotropic and transversely isotropic elastic solids. The solution can be most conveniently formulated in relation to a set of ellipsoidal coordinates $(\xi, \eta, \zeta)$ of 
the point $(x, y, z)$, which are the roots of the cubic equation in $\theta$ defined by

$$
\frac{x^{2}}{\left(a^{2}+\theta\right)}+\frac{y^{2}}{\left(b^{2}+\theta\right)}+\frac{z^{2}}{\theta}-1=0 .
$$

The ellipsoidal coordinate system $(\xi, \eta, \zeta)$ chosen ensures that the interior Dirichlet region $S_{i}$ corresponds to the ellipse $\xi=0$ and the exterior Neumann region $S_{e}$ corresponds to a hyperboloid of one sheet $\eta=0$. The mixed boundary conditions (4) and (5) can be explicitly satisfied by the harmonic function

$$
\Phi(x, y, z)=\frac{a \Phi_{i}}{\mathrm{~K}(\sigma)} \int_{\xi}^{\infty} \frac{d s}{\sqrt{s\left(a^{2}+s\right)\left(b^{2}+s\right)}},
$$

where

$$
\xi=a^{2}\left(\mathrm{sn}^{-2} u-1\right)
$$

and sn $u$ represents the Jacobian elliptic function defined by

$$
\int_{0}^{\mathrm{sn}(u, \sigma)} \frac{d t}{\sqrt{\left(1-t^{2}\right)\left(1-\sigma^{2} t^{2}\right)}}=(u, \sigma) .
$$

In a numerical evaluation of $\operatorname{sn} u$, it is convenient to express the function in the series form:

$$
\begin{aligned}
\operatorname{sn}(u, \sigma)=u-\left(1+\sigma^{2}\right) \frac{u^{3}}{3 !}+(1+ & \left.14 \sigma^{2}+\sigma^{4}\right) \frac{u^{5}}{5 !} \\
& -\left(1+135 \sigma^{2}+135 \sigma^{4}+\sigma^{6}\right) \frac{u^{7}}{7 !}+\cdots .
\end{aligned}
$$

The complete elliptic integral of the first kind $\mathrm{K}(\sigma)$ is defined by

$$
\mathrm{K}(\sigma)=\int_{0}^{\pi / 2} \frac{d \varsigma}{\sqrt{1-\sigma^{2} \sin ^{2} \varsigma}}, \quad \sigma=\left(\frac{a^{2}-b^{2}}{a^{2}}\right)^{1 / 2} .
$$

We can generalize the result (7) to account for the effect of the far-field potential $\Phi_{1}\left(>\Phi_{i}\right)$. This involves simply changing the potential $\Phi_{i}$ in (7) to $\left(\Phi_{1}-\Phi_{i}\right)$. The fluid velocity at the elliptical aperture associated with region 1 is now given by

$$
\begin{aligned}
v_{z}^{(1)}(x, y, 0) & =-\frac{K_{1} \gamma_{w}}{\mu}\left(\frac{\partial \Phi}{\partial z}\right)_{z=0} \\
& =\frac{K_{1} \gamma_{w}\left(\Phi_{1}-\Phi_{i}\right)}{b \mu \mathrm{K}(\sigma)} \frac{1}{\sqrt{1-x^{2} / a^{2}-y^{2} / b^{2}}}, \quad(x, y) \in S_{i},
\end{aligned}
$$

where $K_{1}$ is the permeability of the porous region 1 . The flow rate out of the 
elliptical aperture is given by

$$
Q=\frac{K_{1} \gamma_{w}\left(\Phi_{1}-\Phi_{i}\right)}{\mu b \mathrm{~K}(\sigma)} \iint_{S_{i}} \frac{d x d y}{\sqrt{1-x^{2} / a^{2}-y^{2} / b^{2}}}=\frac{2 \pi a\left(\Phi_{1}-\Phi_{i}\right) \gamma_{w} K_{1}}{\mu \mathrm{K}(\sigma)} .
$$

A similar result can be developed for the potential flow problem where flow takes place from the porous halfspace region 2 at a far-field potential $\Phi_{2}\left(<\Phi_{i}\right)$, which gives the velocity field in the interface approached from region 2 as

$$
\begin{aligned}
v_{z}^{(2)}(x, y, 0) & =-\frac{K_{1} \gamma_{w}}{\mu}\left(\frac{\partial \Phi}{\partial z}\right)_{z=0} \\
& =\frac{K_{1} \gamma_{w}\left(\Phi_{i}-\Phi_{2}\right)}{b \mu \mathrm{K}(\sigma)} \frac{1}{\sqrt{1-x^{2} / a^{2}-y^{2} / b^{2}}}, \quad(x, y) \in S_{i},
\end{aligned}
$$

and the flow rate into region 2 is given by

$$
Q=\frac{2 \pi a\left(\Phi_{i}-\Phi_{2}\right) \gamma_{w} K_{2}}{\mu \mathrm{K}(\sigma)} .
$$

The value of the interface potential $\Phi_{i}$ can be obtained, ensuring continuity of the velocity field at the interface: that is,

$$
v_{z}^{(1)}(x, y, 0)=v_{z}^{(2)}(x, y, 0),
$$

which gives $\Phi_{i}=\left(K_{1} \Phi_{1}+K_{2} \Phi_{2}\right) /\left(K_{1}+K_{2}\right)$. The leakage rate through the elliptical aperture can now be obtained by eliminating $\Phi_{i}$ in either (13) or (15), which gives

$$
Q=\frac{2 \pi a\left(\Phi_{1}-\Phi_{2}\right) \gamma_{w} K_{1} K_{2}}{\mu\left(K_{1}+K_{2}\right) \mathrm{K}(\sigma)} .
$$

It is important to note that the result (17) is the exact closed-form solution for the steady leakage of an incompressible fluid through an elliptical cavity located at the impermeable interface separating isotropic nondeformable porous regions of dissimilar permeability. The potential problem that is solved satisfies the governing equations of potential flow, the mixed boundary conditions applicable to the potential problem and, continuity of both the potential and the flow velocity at the interface where Dirichlet conditions are prescribed. From the uniqueness theorem applicable to mixed boundary value problems in potential theory, this solution is unique [Zauderer 1989, Section 6.8; Selvadurai 2000a, Section 5.7; $2000 \mathrm{~b}$, Section 9.5]. It should be noted that the solution is identical even if a continuity of total flux boundary condition is imposed on the interface rather than a continuity of flow velocity. It is noted from (12) and (14) that although the velocity at the boundary of the elliptical entry region is singular, the volume flow rate to the elliptical cavity region is finite. In the special case when the permeability value of one region becomes large (for example, $K_{2} \rightarrow \infty$ ), (17) reduces to 


$$
Q=\frac{2 \pi a\left(\Phi_{1}-\Phi_{2}\right) \gamma_{w} K_{1}}{\mu \mathrm{K}(\sigma)} .
$$

For the special case when the elliptical opening has the shape of a circular region of radius $a, \mathrm{~K}(0) \rightarrow \pi / 2$ and (18) reduces to the classical result that can be obtained by solving the associated mixed boundary value problem in potential theory for the circular opening at an impervious interface, by appeal to the theory of dual integral equations [Sneddon 1966]. The solution presented for the elliptical defect is valid for all aspect ratios of the defect, which permits the evaluation of leakage rates from narrow cracks. Comparisons of the analytical estimates with computational results are given in [Selvadurai 2012] and the analytical solution provides a benchmark for calibration of computational modeling of the potential problem. It is also worth noting that in instances where the separate porous regions display spatial heterogeneity with a log normal variation in the permeability, which can be characterized by an effective permeability such as the geometric mean [Selvadurai and Selvadurai 2010], the result (17) can be used to estimate the leakage from the elliptical opening.

\section{Fluid leakage from a defect with an arbitrary plan form}

The result (17) can also be used to estimate or develop bounds for the leakage from a damaged region of arbitrary area $A_{D}$, where the bounds for the effective permeability $K_{D}$ are obtained by considering equivalent elliptical regions that either inscribe or circumscribe the region $A_{D}$ (Figure 2), that is,

$$
\bar{Q}_{I} \leq \frac{Q \mu}{2 \pi \gamma_{w}\left(\Phi_{1}-\Phi_{2}\right) \sqrt{A_{D} K_{1} K_{2}}} \leq \bar{Q}_{C}
$$

In (19), $\bar{Q}_{n}(n=I, C)$ denote the nondimensional flow rates, which refer to the elliptical regions that either inscribe $(I)$ or circumscribe $(C)$ the region $A_{D}$ and are given by

$$
\bar{Q}_{n}=\frac{a_{n} \sqrt{K_{1} K_{2}}}{\sqrt{A_{D}}\left(K_{1}+K_{2}\right) \mathrm{K}\left(\sigma_{n}\right)} \quad(n=I, C) .
$$

The nondimensionalization is accomplished by ensuring that the bounds correspond to nondimensional versions derived from (20). Here, $a_{n}$ and $b_{n}(n=I, C)$ are, respectively, the semimajor and semiminor axes of the elliptical region associated with the inscribed and circumscribed ellipses that contain the region of area $A_{D}$, and

$$
\mathrm{K}\left(\sigma_{n}\right)=\int_{0}^{\pi / 2} \frac{d \varsigma}{\sqrt{1-\sigma_{n}^{2} \sin ^{2} \varsigma}}, \quad \sigma_{n}=\left(\frac{a_{n}^{2}-b_{n}^{2}}{a_{n}^{2}}\right)^{1 / 2} \text {. }
$$




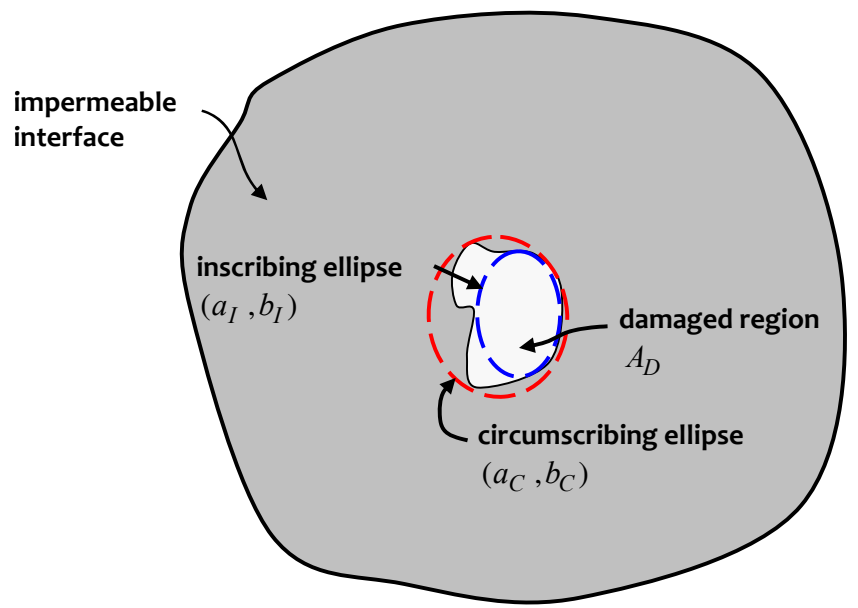

Figure 2. Defect with an arbitrary shape located at an impermeable interface between dissimilar nondeformable porous media.

This approach for bounding the result follows procedures that were proposed by Maxwell [1892] for problems in electrostatics and applied by Pólya and Szegö [1945; 1951] (see also [Protter and Weinberger 1984]) for a variety of problems in potential theory and elastostatics to obtain bounds to problems that are usually regarded as analytically intractable [Galin 1961; Selvadurai 1983].

\section{Fluid leakage from an elliptical defect into a hydraulically transversely isotropic porous medium}

We now consider the problem of a transversely isotropic or stratified porous medium of semiinfinite extent where the stratifications are inclined at an angle $\alpha$ to the surface of the halfspace (Figure 3). The surface of the halfspace is impervious except over an elliptical opening through which fluids can either enter or exit the transversely isotropic porous medium. The orientation of the elliptical opening is such that its minor axis is aligned with the $y$-axis of the spatial coordinate system $(x, y, z)$. To an extent, this is a simplification; otherwise the potential problem would only be amenable to a complicated ellipsoidal harmonic function formulation.

The permeability matrix for the hydraulically transversely isotropic material, $\left[\boldsymbol{K}^{\mathrm{P}}\right]$, referred to the principal directions aligned along the normal $(n)$ and tangential $(t)$ directions, is given by

$$
\left[\boldsymbol{K}^{\mathrm{P}}\right]=\left[\begin{array}{ccc}
K_{t} & 0 & 0 \\
0 & K_{t} & 0 \\
0 & 0 & K_{n}
\end{array}\right],
$$




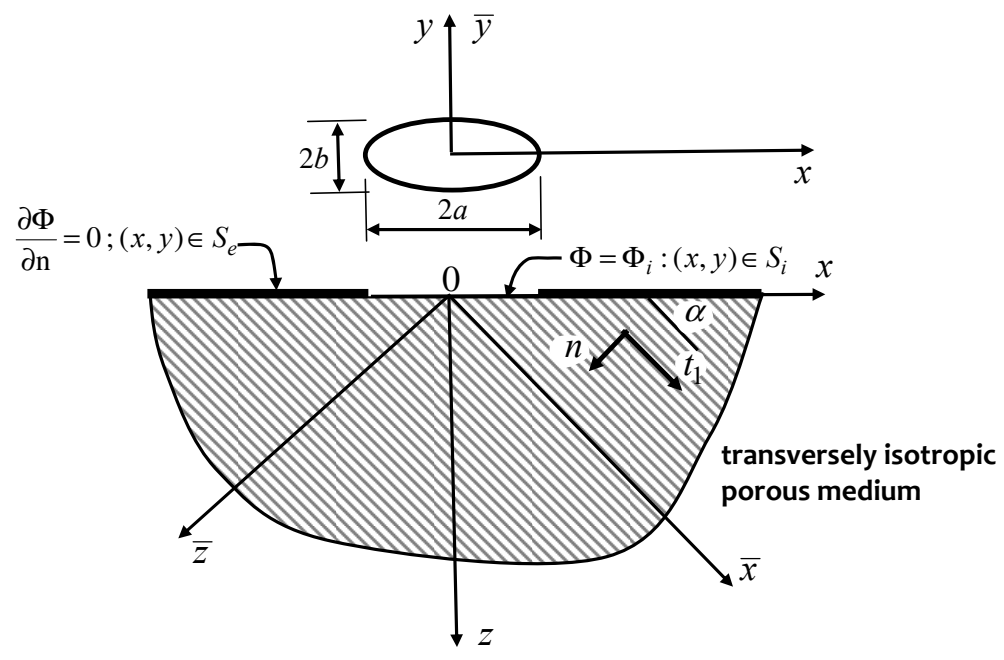

Figure 3. A mixed boundary value problem for flow from an elliptical opening located at the surface of a transversely isotropic porous halfspace.

and the permeability matrix $\left[\boldsymbol{K}^{\mathrm{RC}}\right]$ referred to the rectangular Cartesian coordinate system $(x, y, z)$ is given by

$$
\left[\boldsymbol{K}^{\mathrm{RC}}\right]=[\boldsymbol{H}]^{\mathrm{T}}\left[\boldsymbol{K}^{\mathrm{P}}\right][\boldsymbol{H}],
$$

where

$$
[\boldsymbol{H}]=\left[\begin{array}{ccc}
\cos \alpha & 0 & \sin \alpha \\
0 & 0 & 0 \\
-\sin \alpha & 0 & \cos \alpha
\end{array}\right]
$$

The mixed boundary value problem governing flow into the transversely isotropic elastic halfspace is of the type given by the boundary conditions (4) and (5) indicated previously. It can be shown that this mixed boundary value problem, if formulated in the conventional way, will give rise, even for a circular opening, to a series of dual integral equations of unmanageable complexity. The approach adopted here follows from the transformation technique proposed by Selvadurai [2010], which leads to a manageable problem. Referring to Figure 3, where the principal planes of hydraulic transverse isotropy are aligned with the coordinate system $(\bar{x}, \bar{y}, \bar{z})$, the fluid flow in the transversely isotropic porous medium is given by

$$
K_{t}\left(\frac{\partial^{2} \Phi}{\partial \bar{x}^{2}}+\frac{\partial^{2} \Phi}{\partial \bar{y}^{2}}\right)+K_{n} \frac{\partial^{2} \Phi}{\partial \bar{z}^{2}}=0
$$


At the elliptical region on which the Dirichlet condition is prescribed,

$$
\bar{x}=a \cos \alpha, \quad \bar{y}=b, \quad \bar{z}=a \sin \alpha .
$$

We now make the conventional transformation that converts (25) from a pseudoLaplacian to a Laplacian form, using the following transformations:

$$
\bar{x}=X, \quad \bar{y}=Y, \quad \bar{z}=Z \sqrt{\frac{K_{n}}{K_{t}}},
$$

which reduces (25) to the Laplacian form

$$
\frac{\partial^{2} \Phi}{\partial X^{2}}+\frac{\partial^{2} \Phi}{\partial Y^{2}}+\frac{\partial^{2} \Phi}{\partial Z^{2}}=0
$$

The coordinate directions $X, Y$, and $Z$ are aligned with the axes $\bar{x}, \bar{y}$, and $\bar{z}$, respectively; the new orientation of the halfspace region is defined in relation to the axes $(\tilde{x}, \tilde{y}, \tilde{z})$ and the inclination of the halfspace is defined by $\beta$ as shown in Figure 4. The transformations (27), however, transform the original elliptical cavity with semimajor axis $a$ and semiminor axis $b$ to an ellipse with semimajor axis $c$ and semiminor axis $b$, and the inclination $\beta$ of the plane of the elliptical Dirichlet region at the interface (Figure 4) gives

$$
X=c \cos \beta, \quad Y=b, \quad Z=c \sin \beta .
$$

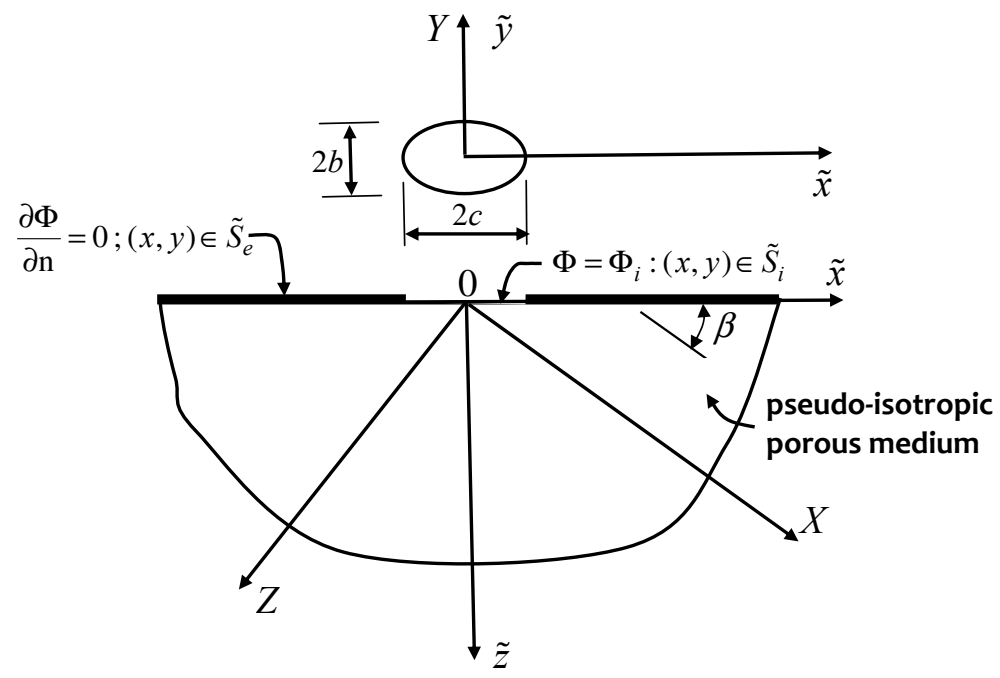

Figure 4. A mixed boundary value problem for flow from an elliptical opening located at the surface of a pseudoisotropic porous medium. 
Using (27) and (29), we obtain

$$
c^{2}=a^{2}\left\{\cos ^{2} \alpha+\frac{K_{t}}{K_{n}} \sin ^{2} \alpha\right\} .
$$

Since the representation of the potential problem in the $(X, Y, Z)$ coordinate system is isotropic, the choice of reference coordinate system to analyze the mixed boundary value problem referred to the Dirichlet boundary conditions prescribed on the transformed elliptical region is arbitrary and it is convenient to select the coordinate system $(\tilde{x}, \tilde{y}, \tilde{z})$ as shown in Figure 4. Referring to this coordinate system, the mixed boundary value problem deals with Dirichlet boundary conditions prescribed within the elliptical opening $\tilde{S}_{i}$ and null Neumann boundary conditions prescribed in the exterior region $\tilde{S}_{e}$, where

$$
\tilde{S}_{i}:\left(\frac{\tilde{x}}{c}\right)^{2}+\left(\frac{\tilde{y}}{b}\right)^{2} \leq 1, \quad \text { and } \quad \tilde{S}_{e}:\left(\frac{\tilde{x}}{c}\right)^{2}+\left(\frac{\tilde{y}}{b}\right)^{2}>1,
$$

where $c$ will be the major axis if $K_{t} / K_{n}>1$ and a minor axis if $K_{t} / K_{n}<1$. Following procedures similar to those outlined in Section 2 it can be shown that the relevant solution for satisfying the mixed boundary conditions

$$
\begin{aligned}
(\Phi)_{\tilde{z}=0} & =\Phi_{i}=\mathrm{constant}, & & (\tilde{x}, \tilde{y}) \in \tilde{S}_{i}, \\
\left(\frac{\partial \Phi}{\partial \tilde{z}}\right)_{\tilde{z}=0} & =0, & & (\tilde{x}, \tilde{y}) \in \tilde{S}_{e},
\end{aligned}
$$

is given by

$$
\Phi(\tilde{x}, \tilde{y}, z)=\frac{c \Phi_{i}}{\mathrm{~K}(\rho)} \int_{\xi}^{\infty} \frac{d s}{\sqrt{s\left(c^{2}+s\right)\left(b^{2}+s\right)}},
$$

where

$$
\rho=\sqrt{\frac{K_{n} \cos ^{2} \alpha+K_{t} \sin ^{2} \alpha-(b / a)^{2}}{K_{n} \cos ^{2} \alpha+K_{t} \sin ^{2} \alpha} .}
$$

The flow rate at the elliptical opening is given by

$$
Q=\frac{2 \pi a \gamma_{w} \Phi_{i} \sqrt{K_{n} K_{t}}}{\mu \mathrm{K}(\rho)} \sqrt{\cos ^{2} \alpha+\frac{K_{t}}{K_{n}} \sin ^{2} \alpha .}
$$

When $K_{t}=K_{n}=K_{0}$, (36) is independent of the orientation $\alpha$ and reduces to a result similar to that given by (18).

The mixed boundary value problem involving dissimilar transversely isotropic porous halfspace regions with planes of transverse isotropy that are arbitrary (see Figure 5) cannot be solved in an exact fashion similar to that outlined previously. This limitation arises from the fact that when both the hydraulic transverse isotropies of the two regions and the inclinations of the principal directions are arbitrary, in the 


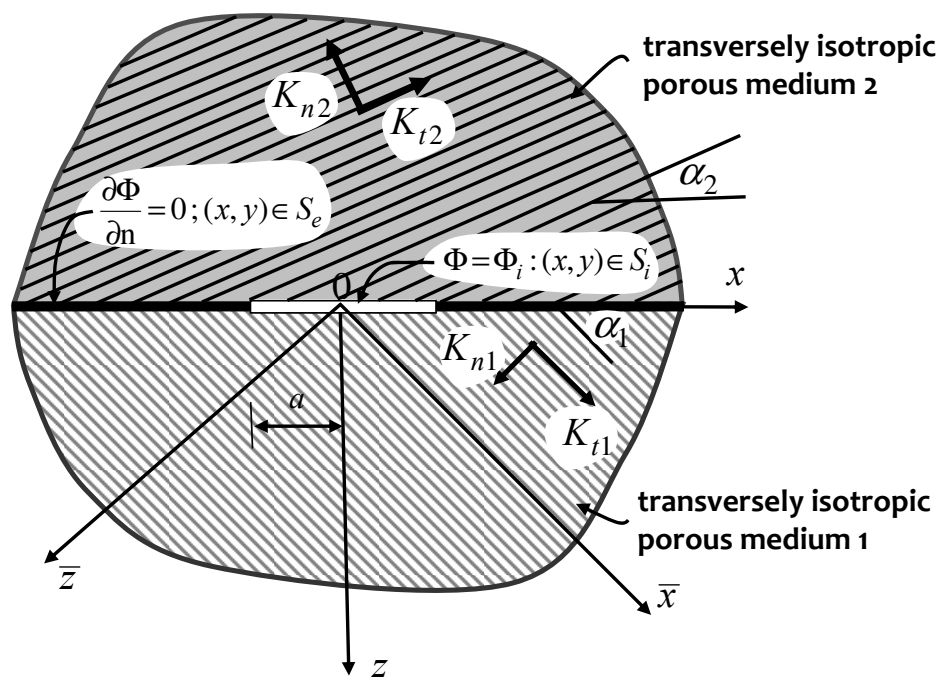

Figure 5. Elliptical defect at an impermeable interface between two dissimilar transversely isotropic porous media.

transformed configuration the dimensions of the ellipses will be different (by virtue of (30)) and will be unequal even if the orientations of the transverse isotropies are made to coincide along one principal direction. An approximate solution to the leakage through an elliptical opening separating dissimilar transversely isotropic regions under far-field potentials $\Phi_{1}$ and $\Phi_{2}$ (with $\Phi_{1}>\Phi_{2}$ ) can be evaluated by assuming that the total flow rate at the elliptical defect is the same for both transversely isotropic regions irrespective of the misalignment in their principal axes.

The flow rate can be estimated from the result

$$
Q \simeq \frac{2 \pi a \gamma_{w}\left(\Phi_{1}-\Phi_{2}\right)}{\mu \sum_{i=1,2} \frac{\mathrm{K}\left(\rho_{i}\right)}{\sqrt{K_{n i} K_{t i}\left\{\cos ^{2} \alpha_{i}+\left(K_{t i} / K_{n i}\right) \sin ^{2} \alpha_{i}\right\}}}}
$$

where

$$
\rho_{i}=\sqrt{\frac{K_{n i} \cos ^{2} \alpha_{i}+K_{t i} \sin ^{2} \alpha_{i}-(b / a)^{2}}{\left(K_{n i} \cos ^{2} \alpha_{i}+K_{t i} \sin ^{2} \alpha_{i}\right)}} .
$$

In the instance when $K_{n 1}=K_{t 1}=K_{1}$ and $K_{n 2}=K_{t 2}=K_{2}$, the solution will be independent of $\alpha_{i}(i=1,2)$ and $\rho_{i}=\sigma$, which is defined by (11). In this case the result (37) reduces to the exact solution given by (17). Similarly as $K_{n 2}=K_{t 2} \rightarrow \infty$, and $K_{n 1}=K_{n}, K_{t 1}=K_{t}$, (37) reduces to the result (36) for a single halfspace. 


\section{Concluding remarks}

The mixed boundary value problem in potential theory for a halfspace region, where Dirichlet boundary conditions are prescribed over an elliptical region and null Neumann boundary conditions are prescribed exterior to the elliptical domain, can be used to develop solutions to porous media flow problems of interest to environmental geosciences. The exact closed-form solutions developed for leakage through an elliptical defect located at an impermeable interface between nondeformable dissimilar porous media can be extended to develop exact closed-form results that can be used to benchmark the accuracy of computational schemes for Darcy flow where singular behavior in the velocity field at the boundary is rarely incorporated in the solution scheme.

\section{Acknowledgements}

The work described in this paper was supported by the 2003 Max Planck Research Award in the Engineering Sciences from the Max-Planck-Gesellschaft, Germany, and through a Discovery Grant awarded by the Natural Sciences and Engineering Research Council of Canada. The author is grateful to the reviewers for providing valuable comments that led to improvements in the paper.

\section{References}

[Bear and Verruijt 1987] J. Bear and A. Verruijt, Modeling groundwater flow and pollution, Reidel, Dordrecht, 1987. Corrected reprint, 1990.

[Galin 1961] L. A. Galin, "Contact problems in the theory of elasticity”, lecture notes, Raleigh, NC, 1961.

[Green and Sneddon 1950] A. E. Green and I. N. Sneddon, "The distribution of stress in the neighbourhood of a flat elliptical crack in an elastic solid", Proc. Cambridge Philos. Soc. 46 (1950), 159-163.

[Kassir and Sih 1968] M. K. Kassir and G. C. Sih, "Some three-dimensional inclusion problems in elasticity”, Int. J. Solids Struct. 4:2 (1968), 225-241.

[Lamb 1927] H. Lamb, Hydrodynamics, 2nd ed., Cambridge Mathematical Library, Cambridge University Press, 1927.

[Maxwell 1892] J. C. Maxwell, A treatise on elasticity and magnetism, Clarendon Press, Oxford, 1892.

[Morse and Feshbach 1953] P. M. Morse and H. Feshbach, Methods of theoretical physics, vol. 2, McGraw-Hill, New York, 1953.

[Phillips 1991] O. M. Phillips, Flow and reactions in permeable rocks, Cambridge University Press, Cambridge, 1991.

[Pólya and Szegö 1945] G. Pólya and G. Szegö, "Inequalities for the capacity of a condenser", Amer. J. Math. 67 (1945), 1-32.

[Pólya and Szegö 1951] G. Pólya and G. Szegö, Isoperimetric inequalities in mathematical physics, Annals of Mathematics Studies 27, Princeton University Press, 1951. 
[Protter and Weinberger 1984] M. H. Protter and H. F. Weinberger, Maximum principles in differential equations, Springer, New York, 1984. corrected reprint.

[Selvadurai 1982] A. P. S. Selvadurai, "Axial displacement of a rigid elliptical disc inclusion embedded in a transversely isotropic elastic solid", Mech. Res. Comm. 9:1 (1982), 39-45.

[Selvadurai 1983] A. P. S. Selvadurai, "Approximations for the low frequency response of a rigid plate embedded in an infinite space", Int. J. Soil Dyn. Earthq. Eng. 2:2 (1983), 78-82.

[Selvadurai 2000a] A. P. S. Selvadurai, Partial differential equations in mechanics, 1: Fundamentals, Laplace's equation, diffusion equation, wave equation, Springer, Berlin, 2000.

[Selvadurai 2000b] A. P. S. Selvadurai, Partial differential equations in mechanics, 2: The biharmonic equation, Poisson's equation, Springer, Berlin, 2000.

[Selvadurai 2010] A. P. S. Selvadurai, "On the hydraulic intake shape factor for a circular opening located at an impervious boundary: influence of inclined stratification", Int. J. Num. Anal. Meth. Geomech. 35:6 (2010), 639-651.

[Selvadurai 2012] A. P. S. Selvadurai, "Fluid leakage through fractures in an impervious caprock embedded between two geologic aquifers", Adv. Water Resour. 41:0 (2012), 76-83.

[Selvadurai and Carnaffan 1997] A. P. S. Selvadurai and P. Carnaffan, "A transient pressure pulse method for the measurement of permeability of a cement grout", Can. J. Civil. Eng. 24 (1997), 489-502.

[Selvadurai and Selvadurai 2010] A. P. S. Selvadurai and P. A. Selvadurai, "Surface permeability tests: experiments and modelling for estimating effective permeability", Proc. Roy. Soc. Ser. A, Math. Phys. Sci. 466:2122 (2010), 2819-2846.

[Selvadurai and Yu 2006a] A. P. S. Selvadurai and Q. Yu, "Constitutive modelling of a polymeric material subjected to chemical exposure”, Int. J. Plasticity 22:6 (2006), 1089-1122.

[Selvadurai and Yu 2006b] A. P. S. Selvadurai and Q. Yu, "On the indentation of a polymeric membrane”, Proc. Roy. Soc. Ser. A, Math. Phys. Sci. 462:2065 (2006), 189-209.

[Selvadurai et al. 2005] A. P. S. Selvadurai, M. J. Boulon, and T. S. Nguyen, "Permeability of an intact granite", Pure and Appl. Geophys. 162 (2005), 373-407.

[Sneddon 1966] I. N. Sneddon, Mixed boundary value problems in potential theory, North-Holland, Amsterdam, 1966.

[Tschebotarioff 1951] G. P. Tschebotarioff, Soil mechanics, foundations, and earth structures: an introduction to the theory and practice of design and construction, McGraw-Hill, New York, 1951.

[Walpole 1991] L. J. Walpole, "A translated rigid ellipsoidal inclusion in an elastic medium", Proc. Roy. Soc. London Ser. A 434:1892 (1991), 571-585.

[Yu and Selvadurai 2005] Q. Yu and A. P. S. Selvadurai, "Mechanical behaviour of a plasticized PVC subjected to ethanol exposure", Polym. Degr. and Stability 89:1 (2005), 109-124.

[Yu and Selvadurai 2007] Q. Yu and A. P. S. Selvadurai, "Mechanics of a rate-dependent polymer network", Phil. Mag. 87:24 (2007), 3519-3530.

[Zauderer 1989] E. Zauderer, Partial differential equations of applied mathematics, 2nd ed., Wiley, 1989.

Received 7 Aug 2012. Revised 13 Mar 2013. Accepted 20 Apr 2013.

A. P. S. SElVAdURAI: patrick.selvadurai@mcgill.ca

Department of Civil Engineering and Applied Mechanics, McGill University,

Macdonald Engineering Building, 817 Sherbrooke Street West, Montréal, QC H3A OC3, Canada 


\title{
GEOMETRIC DEGREE OF NONCONSERVATIVITY
}

\author{
Jean Lerbet, Marwa Aldowaji, Nö̈l Challamel, \\ Oleg N. Kirillov, François Nicot AND FÉlix Darve
}

This paper deals with nonconservative mechanical systems subjected to nonconservative positional forces leading to nonsymmetric tangential stiffness matrices. The geometric degree of nonconservativity of such systems is then defined as the minimal number $\ell$ of kinematic constraints necessary to convert the initial system into a conservative one. Finding this number and describing the set of corresponding kinematic constraints is reduced to a linear algebra problem. This index $\ell$ of nonconservativity is the half of the rank of the skew-symmetric part $K_{a}$ of the stiffness matrix $K$ that is always an even number. The set of constraints is extracted from the eigenspaces of the symmetric matrix $K_{a}^{2}$. Several examples including the well-known Ziegler column illustrate the results.

\section{Introduction}

This paper is concerned with the statics or dynamics of a discrete or discretized system $\Sigma_{\text {free}}$. We assume that, after having started with different possible nonlinear settings, convenient assumptions and approximations lead to a dynamic evolution governed by the following equation of motion of the free system $\Sigma_{\text {free }}$ :

$$
M \ddot{X}+K(p) X=0,
$$

where $K(p)=K_{s}(p)+K_{a}(p)$, with $K_{s}(p)=\frac{1}{2}\left(K(p)+K^{T}(p)\right)$ and $K_{a}(p)=$ $\frac{1}{2}\left(K(p)-K^{T}(p)\right)$; note that $K(p)$ is generally a nonsymmetric matrix $\left(K_{a}(p) \neq 0\right)$ because of the nonconservativity of $\Sigma_{\text {free, }}$, whereas $M$ is symmetric positive definite.

The paradoxical effects of mechanical systems linearly governed by (1) with a nonsymmetric stiffness matrix have been known for some time (see for example [Ziegler 1952; Bolotin 1961]) and have been investigated in depth, especially concerning the so-called destabilizing effect of adding friction in the system (see [Kirillov and Verhulst 2010] for recent developments). Bigoni and Noselli [2011] have illustrated through an experimental device calculations starting from dynamics with dry friction and coming to equations like (1). Circulatory forces seem to have

\section{Communicated by Francesco dell'Isola.}

MSC2010: 15A18, 70G99.

Keywords: linear algebra, nonconservative system. 
appeared first in the rotor dynamics works of early 1920s. It is generally agreed that E. L. Nikolai was the first to have found some curious paradoxes induced by the nonconservative aspect of the loading system. Here, we only investigate systems without rotating effects which means that circulatory forces are only nonconservative positional loading. More precisely, by decomposing in the linear case the stiffness matrix $K(p)$ into a symmetric part and a skew-symmetric part, one then decomposes nonpotential forces into a potential component and a circulatory component. Zhuravlev [2007] suggests an extension of this decomposition for the nonlinear case by using the trick of Poincaré in his theorem about exact and closed differential forms. This could be a good way to tackle the nonlinear extension of the present paper.

If $p$ is a loading parameter any norm $\left\|K_{a}(p)\right\|$ of the skew-symmetric part $K_{a}(p)$ of $K(p)$ is an elementary measure of the nonconservativity of the corresponding nonpotential forces by any norm on the space of matrices. However, this rough measure indicates the amplitude of the nonconservativity and masks another more intrinsic measure of this nonconservativity which is defined in this paper. This is here defined by a lower semicontinuous function with only finite integer values (for an increasing load). This function is then locally independent on the load parameter value, except perhaps for a finite number of singular values $\left\{p_{0}^{*}<p_{1}^{*}<\cdots<p_{r}^{*}\right\}$ of $p$. Obviously $p_{0}^{*}=0$ is such a value, because for $p=0$ the system is conservative and $K_{a}(0)=0$. In all the examples except the so-called Bigoni system, the only value is $p=0$. Because it is also linked to a dimension of a linear space, we then propose to call this number the geometric degree of nonconservativity of the system (or of the forces).

The genesis of the used approach lies in several papers [Challamel et al. 2009; 2010; Nicot et al. 2011; Lerbet et al. 2012] that investigated the deep rule of the second-order work criterion proposed in [Hill 1958] for solids in the framework of nonassociated plasticity, and independently also proposed for instabilities for systems subjected to nonpotential forces in [Absi and Lerbet 2004]. This criterion performs especially well for nonconservative systems because, contrary to the divergence criterion, it remains "stable" under the action of additional kinematics constraints: if this criterion holds for a free system and for a value $p$ of the load parameter, it still holds for the same value $p$ and for a system subjected to any family of additional kinematic constraints. This property, contrary to a similar well-known consequence of the Rayleigh theorems for conservative systems, is generally no more valid for nonconservative systems. This paradoxical behavior of the mechanical system, or more precisely of the stability of the investigated equilibrium configuration of the mechanical system when adding additional kinematic constraints, is actually a characteristic of nonconservative systems.

Thus, extending the above-mentioned works concerning the effects of additional 
constraints on such $n$-DOF (degree of freedom) nonconservative mechanical systems [Challamel et al. 2010; Lerbet et al. 2012; 2013], we focus on families of kinematic constraints that could convert $\Sigma_{\text {free }}$ into a conservative system. More precisely, we address both the problems of the existence of a minimal family (according to the number of constraints) of such constraints and that of building the set of such families. The minimal number of constraints required to convert the nonconservative system $\Sigma_{\text {free }}$ into a conservative system is then a measure of the nonconservativity of $\Sigma_{\text {free }}$ and will be called the geometric degree of nonconservativity of $\Sigma_{\text {free }}$.

The paper is organized as follows: in Section 1, the mechanical problem is reduced to a linear algebra problem. In Section 2, the solution is developed leading to the concept of the geometric degree of nonconservativity of a mechanical system. In Section 3, several examples illustrate the mathematical results.

\section{Modeling of the mechanical problem}

Let $\Sigma_{\text {free }}$ be a $n$-DOF discrete mechanical system and suppose, as above, that the dynamic evolution of $\Sigma_{\text {free }}$ is governed by (1). $X$ is the vector of kinematic unknowns $\left(X^{T}=\left(x_{1}, \ldots, x_{n}\right) \in M_{1 n}(\mathbb{R})\right), M$ is the mass matrix (symmetric definite positive), and $K=K(p)$ is the stiffness matrix. The latter is any square matrix because of the nonconservativity of $\Sigma_{\text {free }}$. Let $p$ be a (loading) parameter. Suppose that $m$ (independent) additional kinematic constraints $C^{1}, \ldots, C^{m}$ are set up on

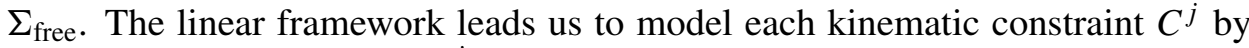
a linear relationship $\sum_{k=1}^{n} \alpha_{k}^{j} x_{k}=0$. Thus $C^{j}$ is represented by and identified with a vector $\alpha^{j}=\left(\alpha_{1}^{j}, \ldots, \alpha_{n}^{j}\right)$ of $\mathbb{R}^{n}$ (actually it is a linear form on $\mathbb{R}^{n}$ but by the canonical scalar product we may identify both spaces). The family of $m$ constraints $\left\{\alpha^{1}, \ldots, \alpha^{m}\right\}$ may be considered as an element of an $\mathrm{nm}$-dimensional vector space - for instance as an $n \times m$ matrix $A=\left(\begin{array}{llll}\alpha^{1} & \cdots & \alpha^{m}\end{array}\right)$ in $\mathcal{M}_{n m}(\mathbb{R})$, or more precisely in $\varphi_{n m}(\mathbb{R})$, the open subset of matrices of $\mathcal{M}_{n m}(\mathbb{R})$ with rank $m$, because of the independence of the constraints. If $m$ is fixed (it will have to be found in a first step), we then have to find the set $C_{m}\left(\Sigma_{\text {free }}\right) \subset \mathscr{G}_{n m}(\mathbb{R})$ such that if $A \in C_{m}\left(\Sigma_{\text {free }}\right)$ then the constrained mechanical system $\Sigma_{\text {cons }}=\Sigma_{\text {cons }}(A)$ becomes conservative. Thus

$$
A^{T}=\left(\begin{array}{c}
\alpha^{1 T} \\
\vdots \\
\alpha^{m T}
\end{array}\right)=\left(\begin{array}{ccc}
\alpha_{1}^{1} & \cdots & \alpha_{n}^{1} \\
\vdots & \ddots & \vdots \\
\alpha_{1}^{m} & \cdots & \alpha_{n}^{m}
\end{array}\right)
$$

(every vector $\alpha^{i T}=\left(\alpha_{1}^{i}, \ldots, \alpha_{n}^{i}\right)$ could be normalized $\alpha^{i T} \alpha^{i}=1$ ).

Let $\Lambda \in M_{m 1}(\mathbb{R})$, with $\Lambda^{T}=\left(\lambda_{1} \ldots \lambda_{m}\right)$, be the Lagrange multiplier attached to the constraints. The equation of motion of the constrained system $\Sigma_{\text {cons }}(A)$ is 


$$
\begin{aligned}
A^{T} X & =0, \\
M \ddot{X}+K(p) X+A \Lambda & =0,
\end{aligned}
$$

Let $T(A)=\operatorname{Vect}\left\{\alpha^{1}, \ldots, \alpha^{m}\right\}$ and let $H(A)=T(A)^{\perp}$ be the orthogonal to $T(A)$ in $\mathbb{R}^{n}$ identified with $\mu_{n 1}(\mathbb{R})$. Thus $\operatorname{dim} T(A)=m$ and $\operatorname{dim} H(A)=n-m$.

Let us choose an orthonormal basis of $T(A)$ (by Gram-Schmidt from $\left(\alpha^{1}, \ldots\right.$, $\left.\alpha^{m}\right)$, for example) $\left(t_{1}(A), \ldots, t_{m}(A)\right)$ and another $\left(h_{m+1}(A), \ldots, h_{n}(A)\right)$ of $H(A)$ such that $b(A)=\left(t_{1}(A), \ldots, t_{m}(A), h_{m+1}(A), \ldots, h_{n}(A)\right)$ is an orthonormal basis of $\mathbb{R}^{n}$ and let $P=P(A) \in O_{n}(\mathbb{R})$ be the orthogonal matrix passing from the canonical basis of $\mathbb{R}^{n}$ to $b(A)$ :

$$
P=P(A)=\operatorname{mat}\left(t_{1}(A), \ldots, t_{m}(A), h_{m+1}(A), \ldots, h_{n}(A)\right) .
$$

Let $Y$ be defined by $X=P(A) Y$. The previous system reads:

$$
\begin{aligned}
\left(P(A)^{T} A\right)^{T} Y & =0, \\
P^{T}(A) M P(A) \ddot{Y}+P^{T}(A) K(p) P(A) Y+P(A)^{T} A \Lambda & =0,
\end{aligned}
$$

Considering $M_{\text {cons }}(A)$ (resp. $K_{\text {cons }}(A, p)$ ) the square submatrix of $P^{T}(A) M P(A)$ (resp. $\left.P^{T}(A) K(p) P(A)\right)$ built by suppressing the first $m$ rows and the first $m$ columns of $P^{T}(A) M P(A)$ (resp. of $P^{T}(A) K(p) P(A)$ ), we get the following equations of the constrained system without the Lagrange multipliers:

$$
M_{\text {cons }}(A) \ddot{Y}_{\text {cons }}+K_{\text {cons }}(A, p) Y_{\text {cons }}=0,
$$

where $Y_{\text {cons }}^{T}=\left(y_{m+1}, \ldots, y_{n}\right) \in M_{1 n-m}(\mathbb{R})$.

We are then led to investigate when $K_{\text {cons }}(A, p)$, a $(n-m) \times(n-m)$ square submatrix of $P^{T}(A) K(p) P(A)$, is symmetric. Note that, in the standard case of structural mechanics, $K(p)=K_{\mathrm{el}}-p K_{\mathrm{ext}}$ with $K_{\mathrm{el}}$ the symmetric definite-positive stiffness matrix relative to elastic actions and $K_{\text {ext }}$ the nonsymmetric matrix relative to external actions (circulatory force). $K_{\text {cons }}(A, p)$ reads:

$$
K_{\text {cons }}(A, p)=\left(\begin{array}{ccc}
h_{m+1}^{T}(A) K(p) h_{m+1}(A) & \cdots & h_{m+1}^{T}(A) K(p) h_{n}(A) \\
\vdots & \ddots & \vdots \\
h_{n}^{T}(A) K(p) h_{m+1}(A) & \cdots & h_{n}^{T}(A) K(p) h_{n}(A)
\end{array}\right) .
$$

The condition for the constraints defined by $A$ to convert the free nonconservative system into a conservative one is then

$$
h_{i}^{T}(A) K(p) h_{j}(A)=h_{i}^{T}(A) K^{T}(p) h_{j}(A) \text { for all } i, j,
$$

or, in a more geometrical phrasing: for every pair $u$ and $v$ of two orthogonal vectors of $H(A)$ orthogonal to $T(A)=\operatorname{Vect}\left\{\alpha^{1}, \ldots, \alpha^{m}\right\}, u^{T} K(p) v=u^{T} K^{T}(p) v$. This 
is equivalent to $u^{T} K_{a}(p) v=0$ for every pair $u$ and $v$ of any two vectors of $H(A)$ with $K_{a}(p)$ the skew-symmetric part of $K(p)$. This is obviously right for $m=n-1$ because for any $n-1$ independent constraints the submatrix becomes a scalar, the skew-symmetric part of which is always nil!

Let $\phi_{a}(p)$ be the linear map whose matrix is in the canonical basis of $\mathbb{R}^{n}$ is $K_{a}(p)$. Geometrically, the condition means that for every vector $u$ of $H(A)$, $\phi_{a}(p)(u)$ is orthogonal to $H(A)$ or equivalently belongs to $T(A)$ :

$$
\phi_{a}(p)(u) \in T(A) \text { for all } u \in H(A) .
$$

The initial mechanical problem has then been modeled into the following original problem of linear algebra and more precisely of Euclidean spaces: Does there exist an $(n-m)$-dimensional subspace of $\mathbb{R}^{n}$ which is sent onto its orthogonal by $\phi_{a}(p)$ ? We denote by $(\cdot \mid \cdot)$ the scalar product of $\mathbb{R}^{n}\left((u \mid v)=u^{T} v\right.$ with the identification of $\mathbb{R}^{n}$ with $\left.\mu_{n 1}(\mathbb{R})\right)$. In the following section, this problem is solved.

\section{Solution of the mathematical modeling}

We forget the $p$-dependency of all the quantities. In the introduction, we already noted that the loading interval $I=\left[0,+\infty[\right.$ may be decomposed as $\left.\{0\} \cup] 0, p_{1}^{*}\right] \cup$ ]$\left.\left.p_{1}^{*}, p_{2}^{*}\right] \cup \cdots \cup\right] p_{r}^{*},+\infty\left[\right.$ with $p_{1}^{*}, \ldots, p_{r}^{*}$ nonzero singular values of loading. Forgetting the $p$-dependency means that $p \notin\left\{0, p_{1}^{*}, \ldots, p_{r}^{*}\right\}$. In the examples excepted for Bigoni's system, 0 is the only singular value. The singular problem is not investigated in this paper.

Let $F_{a}=\operatorname{Im}\left(\phi_{a}\right)$ and $G_{a}=\operatorname{Ker}\left(\phi_{a}\right)$. We know that (as every skew-symmetric linear map) $\phi_{a}$ has an even rank, $r=2 \ell$, and that its kernel and its image are orthogonal spaces. Let $G_{a}=\operatorname{Ker}\left(\phi_{a}\right)$. Thus $\mathbb{R}^{n}=F_{a} \oplus G_{a}$. We set the following:

Definition. The integer $\ell$ is called the geometric degree of nonconservativity of $\Sigma_{\text {free }}$.

As $\phi_{a}^{2}$ is a symmetric linear mapping it is diagonalizable in an orthonormal basis. Moreover $G_{a}=\operatorname{Ker}\left(\phi_{a}\right)=\operatorname{Ker}\left(\phi_{a}^{2}\right)$, the nonzero eigenvalues of $\phi_{a}^{2}$ are negative, and the associated eigenspaces are two-dimensional and mutually orthogonal. Note these values $-\mu_{1}^{2}, \ldots,-\mu_{\ell}^{2}$ and $E_{-\mu_{i}^{2}}$, the associated eigenspaces for $i=1, \ldots, \ell$. Each of these spaces are $\phi_{a}$-stable. Because of the $\phi_{a}$-stability of each of the spaces of the decomposition

$$
\mathbb{R}^{n}=G_{a} \stackrel{\perp}{\oplus} E_{-\mu_{1}^{2}} \stackrel{\perp}{\oplus} \ldots \stackrel{\perp}{\oplus} E_{-\mu_{\ell}^{2}}
$$

we deduce (by Cartan's theorem) the existence of an orthonormal basis $b^{\prime}$ of $\mathbb{R}^{n}$ 
such that the matrix of $\phi_{a}$ in $b^{\prime}$ is

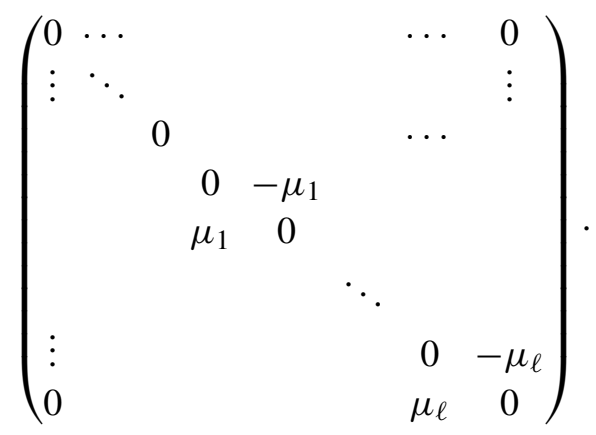

Thus

$$
\mathbb{R}^{n}=G_{a} \stackrel{\perp}{\oplus} E_{-\mu_{1}^{2}} \stackrel{\perp}{\oplus} \cdots \stackrel{\perp}{\oplus} E_{-\mu_{\ell}^{2}}=F_{a} \stackrel{\perp}{\oplus} G_{a}=H(A) \stackrel{\perp}{\oplus} T(A) .
$$

Proposition. Equation (7) holds if and only if $m=\ell$ and the family $A$ of constraints must be built by choosing $\alpha^{i} \in E_{-\mu_{i}^{2}}$ for $i=1, \ldots, \ell=m$, being, however, careful that the property fails if two constraints are chosen in the same eigenspace $E_{-\mu_{i}^{2}}$. Proof. Suppose first that $m=\ell=\frac{1}{2} \operatorname{rank}\left(\phi_{a}\right)$ and $A$ built as proposed in the proposition. Let $u \in H(A)$. Complete the basis $\left(\alpha^{1}, \ldots, \alpha^{m}\right)$ by $n-2 m$ vectors $\beta^{1}, \ldots, \beta^{n-2 m}$ of $G_{a}$ and the $m$ other vectors $\left(\phi_{a}\left(\alpha^{1}\right), \ldots, \phi_{a}\left(\alpha^{m}\right)\right)$ so that the family $\left(\beta^{1}, \ldots \beta^{n-2 m}, \phi_{a}\left(\alpha^{1}\right), \ldots, \phi_{a}\left(\alpha^{m}\right)\right)$ is an orthogonal basis of $T(A)$, as may be easily checked. By definition,

$$
u=\sum_{k=1}^{m} u_{k} \alpha^{k}
$$

and then

$$
\phi_{a}(p)(u)=\sum_{k=1}^{m} u_{k} \phi\left(\alpha^{k}\right) \in T(A),
$$

which is exactly (7).

Reciprocally, suppose now $m<l$ or $m=l$ but $A$ is not built as proposed in the proposition. Thus there is some $i \in\{1, \ldots, l\}$ such that any $\alpha^{j}$ belongs to $E_{-\mu_{i}^{2}}$ meaning geometrically that $T(A) \cap E_{-\mu_{i}^{2}}=\{0\}$. Choose now $u \neq 0$ in $H(A) \cap E_{-\mu_{i}^{2}}$. Thus $\left(u, \phi_{a}(u)\right)$ is an orthogonal basis of $E_{-\mu_{i}^{2}}$, meaning that $\phi_{a}(u)(\neq 0) \in E_{-\mu_{i}^{2}}$, implying $\phi_{a}(u) \notin T(A)$.

Thus, coming back to the mechanical problem, a free nonconservative mechanical system $\Sigma_{\text {free }}$ can be made conservative by means of $m$ constraints if and only if $K_{a}(p)$ has rank $2 m$ and the matrix $A$ is formed by by $m$ vectors $\alpha^{1}, \ldots, \alpha^{m}$, each $\alpha^{i}$ being chosen in the eigenspace $E_{-\mu_{i}^{2}}$ of $K_{a}(p)^{2}$; and $C_{m}(\Sigma)=\left\{A \in \mathcal{M}_{n m}(\mathbb{R}) \mid\right.$ $\left.\operatorname{col}_{i}(A) \in E_{-\mu_{i}^{2}} \backslash\{0\}\right\}$ (with obvious notations) is an open $2 m$-dimensional cone of $\mu_{n m}(\mathbb{R})$. 
As stated previously, any constrained conservative system is still a conservative system. Thus if there are $k \geq m$ constraints, and $m$ of the $k$ constraints are chosen as above, the constrained system is still conservative. If $\operatorname{rank}\left(K_{a}(p)\right)=2 m$, then $m$ is the minimum number of constraints needed to convert the system into a conservative one. The nonconservativity of the free system $\Sigma_{\text {free }}$ is then characterized by two measures of nonconservativity. The first is the norm of the skew-symmetric part $K_{a}(p)$, which indicates the amplitude of the nonconservativity, while the second is the rank $2 \ell$ of $K_{a}(p)$, which acts as a geometric measure of the nonconservativity or a sort of dimension $(\ell)$ of the nonconservativity. This is the reason for the above definition of the geometric degree of nonconservativity of $\Sigma_{\text {free. }}$. Moreover we may localize this nonconservativity because we may build families of $\ell$ vectors (or constraints) allowing us to convert the initial nonconservative system into a conservative one. Note also that the proof is constructive because it builds the kinematic constraints $A$ converting the system $\Sigma_{\text {free }}$ into a conservative one $\left(\Sigma_{\text {cons }}(A)\right)$. There are $2^{m}$ different independent systems $A$ of constraints converting the nonconservative $\Sigma_{\text {free }}$ into a conservative $\Sigma_{\text {cons }}(A)$. This result may be considered as a sort of dual to the result about the destabilizing effect of adding kinematic constraints in nonconservative systems (see again [Challamel et al. 2009; 2010; Nicot et al. 2011; Lerbet et al. 2012]): by adding a suitable constraint in a suitable eigenspace of $K_{s}$, one can destabilize a stable nonconservative system. Here, by choosing appropriate constraints in suitable eigenspaces of $K_{a}^{2}$, one can convert a nonconservative system into a conservative one. In the following section, several examples issued from different mechanical systems illustrate these results.

\section{Examples}

In this section, we propose a collection of examples consisting in variations on the paradigmatic Ziegler column. The degree of freedom (parameter $n$ ) and the nature of the follower force (partial or complete follower force parameter $\gamma$ ) may change. In the most general case, the system $\Sigma$ consists of $n$ bars $O A_{1}, A_{1} A_{2}, \ldots, A_{n-1} A_{n}$ with $O A_{1}=A_{1} A_{2}=\cdots=A_{n-1} A_{n}=h$ linked with $n$ elastic springs with the same stiffness $k . \vec{P}$ is the follower nonconservative load acting on $A_{n}$. Adopting a dimensionless format, we use $p=\|\vec{P}\| h / k$ as a loading parameter. To investigate how the algebraic method is performing, we conduct the complete calculation only for the three-DOF Ziegler column.

In Section 3.1, we investigate the pure Ziegler system and we notice that the geometric degree of nonconservativity is one for any number of rigid bars, meaning for any degree of freedom. Increasing the number of bars or the degree of freedom does not change its geometric degree of nonconservativity: from the geometric point of view, the Ziegler system is weakly nonconservative. In Section 3.2, we 
investigate what we call the multiple-DOF Bigoni system, because it involves device like that of [Bigoni and Noselli 2011] at each joint. This system appears as a generalization of the $n$-DOF Ziegler column where the load parameter is itself distributed on the system and may vary on each joint. It also may be considered as a discretized Leipholz column [Leipholz 1987]. In this case, the geometric degree of nonconservativity increases with the number of bars and the degree of freedom. Calculations are made only for $n=2$ and $n=4$. From a geometric point of view, the Bigoni system is essentially more strongly nonconservative than the Ziegler system.

\subsection{Ziegler systems.}

3.1.1. Two-DOF Ziegler column with complete follower force. The geometric stiffness matrix is

$$
K_{\mathrm{ext}}=\left(\begin{array}{rr}
1 & -1 \\
0 & 0
\end{array}\right)
$$

Its skew-symmetric part is

$$
K_{a, \text { ext }}=\left(\begin{array}{rr}
0 & -\frac{1}{2} \\
\frac{1}{2} & 0
\end{array}\right) .
$$

The square of $K_{a \text {,ext }}$ is

$$
K_{a, \mathrm{ext}}^{2}=-\frac{1}{4}\left(\begin{array}{ll}
1 & 0 \\
0 & 1
\end{array}\right),
$$

where $\mu_{1}^{2}=-\frac{1}{4}, K_{a, \text { ext }}^{2}$ is spheric, and $E_{\mu_{1}}\left(\phi_{a, \text { ext }}^{2}\right)=\mathbb{R}^{2}$. Then $\alpha$ is any vector in $\mathbb{R}^{2}$. Obviously any constraint converts the free system into a conservative one as a one-DOF (elastic) system is always conservative because any continuous function has a primitive.

3.1.2. Two-DOF Ziegler column with partial follower force. The geometric stiffness matrix is

$$
K_{\mathrm{ext}}=\left(\begin{array}{cc}
1 & -\gamma \\
0 & 1-\gamma
\end{array}\right)
$$

Its skew-symmetric part is

$$
K_{a, \mathrm{ext}}=\left(\begin{array}{rr}
0 & -\frac{\gamma}{2} \\
\frac{\gamma}{2} & 0
\end{array}\right) .
$$

The square of $K_{a \text {,ext }}$ is

$$
K_{a, \mathrm{ext}}^{2}=-\frac{\gamma^{2}}{4}\left(\begin{array}{ll}
1 & 0 \\
0 & 1
\end{array}\right) .
$$

Our conclusions are similar to those above. 
3.1.3. Three-DOF Ziegler column with complete follower force. The geometric stiffness matrix is

$$
K_{\mathrm{ext}}=\left(\begin{array}{rrr}
1 & 0 & -1 \\
0 & 1 & -1 \\
0 & 0 & 0
\end{array}\right)
$$

Its skew-symmetric part is

$$
K_{a, \mathrm{ext}}=\frac{1}{2}\left(\begin{array}{rrr}
0 & 0 & -1 \\
0 & 0 & -1 \\
1 & 1 & 0
\end{array}\right) .
$$

Obviously $\operatorname{rank}\left(K_{a, \text { ext }}\right)=2$. The square of $K_{a, \text { ext }}$ is:

$$
K_{a, \mathrm{ext}}^{2}=-\frac{1}{4}\left(\begin{array}{lll}
1 & 1 & 0 \\
1 & 1 & 0 \\
0 & 0 & 2
\end{array}\right) \text {. }
$$

Calculations give $-\mu_{1}^{2}=-\frac{1}{2}$ and

$$
E_{-\frac{1}{2}}\left(K_{a, \text { ext }}^{2}\right)=\operatorname{Vec}\left\{\alpha=\left(\begin{array}{l}
0 \\
0 \\
1
\end{array}\right), K_{a, \text { ext }} \alpha=\frac{1}{2}\left(\begin{array}{r}
-1 \\
-1 \\
0
\end{array}\right)\right\},
$$

leading to two generic constraints converting the system into a conservative one: $\theta_{3}=0$ and $\theta_{1}+\theta_{2}=0$. In practice, any linear combination of these two constraints lies in the corresponding plane and may be chosen as a possible constraint converting the system into a conservative one. We now propose to check for this case the results coming from our algebraic method with respect to the direct approach to the problem.

The virtual power of the follower force reads:

$$
\mathscr{P}^{*}(P)=Q_{1} \theta_{1}^{*}+Q_{2} \theta_{2}^{*}+Q_{3} \theta_{3}^{*}=P h\left(\sin \left(\theta_{3}-\theta_{1}\right) \theta_{1}^{*}+\sin \left(\theta_{3}-\theta_{2}\right) \theta_{2}^{*}\right) .
$$

The complete nonlinear condition in order to have a conservative system is that there is a function $\theta=\left(\theta_{1}, \theta_{2}, \theta_{3}\right) \mapsto U\left(\theta_{1}, \theta_{2}, \theta_{3}\right)=U(\theta)$ such that $Q_{k}=-\partial U / \partial \theta_{k}$, which is here obviously impossible without an additional constraint: that the free system is nonconservative!

Suppose now the system is subjected to a kinematic constraint $\phi(\theta)=0$, which leads to the following condition on the virtual parameters:

$$
\frac{\partial \phi}{\partial \theta_{1}} \theta_{1}^{*}+\frac{\partial \phi}{\partial \theta_{2}} \theta_{2}^{*}+\frac{\partial \phi}{\partial \theta_{3}} \theta_{3}^{*}=0
$$

Supposing the problem is resolvable with respect to the variable $\theta_{3}$, meaning that $\partial \phi / \partial \theta_{3} \neq 0$, we deduce from the implicit functions theorem that (locally in the 
neighborhood of $\theta=0), \theta_{3}=\theta_{3}\left(\theta_{1}, \theta_{2}\right)$ meaning that, to first order,

$$
\theta_{3}=\left.\theta_{3}\left(\theta_{1}, \theta_{2}\right) \approx \frac{\partial \theta_{3}}{\partial \theta_{1}}\right|_{\theta=0} \theta_{1}+\left.\frac{\partial \theta_{3}}{\partial \theta_{2}}\right|_{\theta=0} \theta_{2}=c_{1} \theta_{1}+c_{2} \theta_{2}
$$

Thus, to first order, the expansion reads: $Q_{1}=Q_{1}\left(\theta_{1}, \theta_{2}\right) \approx P h\left(\left(c_{1}-1\right) \theta_{1}+c_{2} \theta_{2}\right)$ and $Q_{2}=Q_{2}\left(\theta_{1}, \theta_{2}\right) \approx \operatorname{Ph}\left(\left(c_{1} \theta_{1}+\left(c_{2}-1\right) \theta_{2}\right)\right.$. The condition of conservativity of the loading then reads $c_{1}=c_{2}=c$. The kinematic relation is $\theta_{3}=c\left(\theta_{1}+\theta_{2}\right)$ and the quadratic potential is

$$
U(\theta) \approx-P h\left(\frac{c-1}{2}\left(\theta_{1}^{2}+\theta_{2}^{2}\right)+c \theta_{1} \theta_{2}\right) .
$$

For $c=0$, we find the first generic kinematic constraint $\theta_{3}=0$, and for $c \neq 0$ it is, as expected, a linear combination of both generic constraints.

Suppose now that the problem is not resolvable with respect to the variable $\theta_{3}$, meaning that $\partial \phi / \partial \theta_{3}=0$. We then deduce that, linearly, the relation only concerns $\theta_{1}$ and $\theta_{2}$ and reads linearly as

$$
\left.\frac{\partial \phi}{\partial \theta_{1}}\right|_{\theta=0} \theta_{1}+\left.\frac{\partial \phi}{\partial \theta_{2}}\right|_{\theta=0} \theta_{2}=a_{1} \theta_{1}+a_{2} \theta_{2} \approx 0,
$$

and that $a_{1} \theta_{1}^{*}+a_{2} \theta_{2}^{*}=0$. Resolving these relations, for example, with respect to the variable $\theta_{1}\left(\theta_{1}=-b \theta_{2}=-\left(a_{2} / a_{1}\right) \theta_{2}\right.$ and reporting this relation in (8) shows that $\mathscr{P}^{*}(P)=Q_{2} \theta_{2}^{*}$ with $Q_{2}=Q_{2}\left(\theta_{2}, \theta_{3}\right) \approx P h\left(-b\left(\theta_{3}-b \theta_{2}\right)+\left(\theta_{3}-\theta_{2}\right)\right) \approx$ $P h\left(\left(b^{2}-1\right) \theta_{2}+(1-b) \theta_{3}\right)$. The condition of integrability then reads $b=1$, the kinematic relation is $\theta_{1}+\theta_{2}=0$, and the potential is nil up to order two. Then we will come back precisely to the second generic kinematic constraint. To sum up, the direct calculations lead to both generic constraints obtained from our algebraic method.

3.1.4. Three-DOF Ziegler column with partial follower force. The geometric stiffness matrix is

$$
K_{\mathrm{ext}}=\left(\begin{array}{ccc}
1 & 0 & -\gamma \\
0 & 1 & -\gamma \\
0 & 0 & 1-\gamma
\end{array}\right)
$$

Its skew-symmetric part is

$$
K_{a, \mathrm{ext}}=\frac{1}{2} \gamma\left(\begin{array}{rrr}
0 & 0 & -1 \\
0 & 0 & -1 \\
1 & 1 & 0
\end{array}\right)
$$


Obviously $\operatorname{rank}\left(K_{a, \text { ext }}\right)=2$. The square of $K_{a \text {,ext }}$ is

$$
K_{a, \text { ext }}^{2}=-\frac{1}{4} \gamma^{2}\left(\begin{array}{lll}
1 & 1 & 0 \\
1 & 1 & 0 \\
0 & 0 & 2
\end{array}\right)
$$

Calculations give $-\mu_{1}^{2}=-\gamma^{2} / 2$ and

$$
E_{-\gamma^{2} / 2}\left(K_{a, \text { ext }}^{2}\right)=\operatorname{Vec}\left\{\alpha=\left(\begin{array}{l}
0 \\
0 \\
1
\end{array}\right), K_{a, \text { ext }} \alpha=\frac{\gamma}{2}\left(\begin{array}{r}
-1 \\
-1 \\
0
\end{array}\right)\right\}
$$

leading to the same two generic constraints as previously which convert the system into a conservative one: $\theta_{3}=0$ and $\theta_{1}+\theta_{2}=0$.

3.1.5. An $n$-DOF Ziegler column with complete follower force $(\gamma=1)$. The stiffness matrix is

$$
K(p)=\left(\begin{array}{ccccccc}
2-p & -1 & 0 & 0 & \cdots & 0 & p \\
-1 & 2-p & -1 & 0 & \cdots & 0 & p \\
0 & -1 & 2-p & -1 & \cdots & 0 & p \\
\vdots & \vdots & \vdots & \vdots & \ddots & \vdots & \vdots \\
0 & 0 & 0 & 0 & \cdots & 2-p & -1+p \\
0 & 0 & 0 & 0 & \cdots & -1 & 1
\end{array}\right) .
$$

Its skew-symmetric part is

$$
K_{a}(p)=\frac{p}{2}\left(\begin{array}{ccccccc}
0 & 0 & 0 & 0 & \cdots & 0 & 1 \\
0 & 0 & 0 & 0 & \cdots & 0 & 1 \\
0 & 0 & 0 & 0 & \cdots & 0 & 1 \\
\vdots & \vdots & \vdots & \vdots & \ddots & \vdots & \vdots \\
0 & 0 & 0 & 0 & \cdots & 0 & 1 \\
-1 & -1 & -1 & -1 & \cdots & -1 & 0
\end{array}\right)
$$

Obviously $\operatorname{rank}\left(K_{a}(p)\right)=2$. The square of $K_{a}(p)$ is

$$
K_{a}^{2}(p)=-\frac{p^{2}}{4}\left(\begin{array}{ccccccc}
1 & 1 & 1 & 1 & \cdots & 1 & 0 \\
1 & 1 & 1 & 1 & \cdots & 1 & 0 \\
1 & 1 & 1 & 1 & \cdots & 1 & 0 \\
\vdots & \vdots & \vdots & \vdots & \ddots & \vdots & \vdots \\
1 & 1 & 1 & 1 & \cdots & 1 & 0 \\
0 & 0 & 0 & 0 & \cdots & 0 & n-1
\end{array}\right)=p^{2} \widetilde{K}_{a}^{2} .
$$



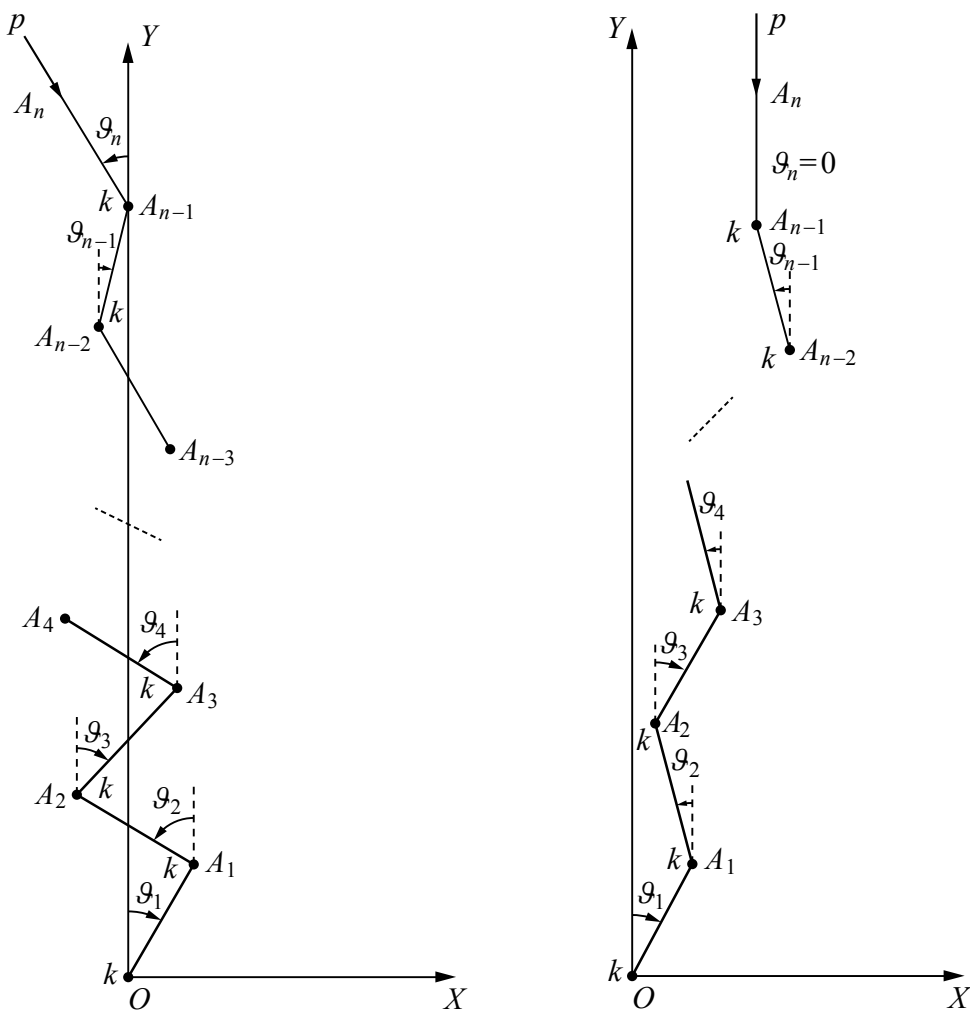

Figure 1. An $n$-DOF Ziegler column with complete follower force: the case $\theta_{1}+\cdots+\theta_{n-1}=0$ (left) and the case $\theta_{n}=0$ (right).

Calculations give $-\mu_{1}^{2}=-(n-1) / 4$ and

$$
E_{-(n-1) / 4}\left(\widetilde{K}_{a}^{2}\right)=\operatorname{Vec}\left\{\alpha=\left(\begin{array}{c}
0 \\
0 \\
0 \\
\vdots \\
0 \\
1
\end{array}\right), \widetilde{K}_{a}^{2} \alpha=\frac{1}{2}\left(\begin{array}{c}
-1 \\
-1 \\
-1 \\
\vdots \\
-1 \\
0
\end{array}\right)\right\}
$$

leading to two generic constraints converting the system into a conservative one: $\theta_{1}+\cdots+\theta_{n-1}=0$, meaning that the motion of $A_{n-1}$ is constrained to remain on the axis $O Y$ (Figure 1, left), and $\theta_{n}=0$ (Figure 1, right).

3.2. The Bigoni system or discretized Leipholz column. We now turn to the $n$ DOF Bigoni system [Bigoni and Noselli 2011], which can also be regarded as an $n$-DOF Leipholz column [1987]. The system $\Sigma$ consists of $n$ bars $O A_{1}, A_{1} A_{2}$, 
$\ldots, A_{n-1} A_{n}$, with $O A_{1}=A_{1} A_{2}=\cdots=A_{n-1} A_{n}=h$ linked with $n$ elastic springs with the same stiffness $k$. Adopting the same device at the end of each bar of $\Sigma$ leads to a family of follower forces $\vec{P}_{1}, \ldots, \vec{P}_{n}$ (see Figure 2 , left). The pure follower forces $\vec{P}_{1}, \vec{P}_{2}, \ldots, \vec{P}_{n}$ are applied at the ends of $O A_{1}, A_{1} A_{2}, \ldots, A_{n-1} A_{n}$, respectively. Adopting a dimensionless format, we use $p_{i}=\left\|\vec{P}_{i}\right\| h / k$, for $i=$ $1, \ldots, n$, as loading parameters. The stiffness matrix is $K(p)=K\left(p_{1}, p_{2}, \ldots, p_{n}\right)$ :

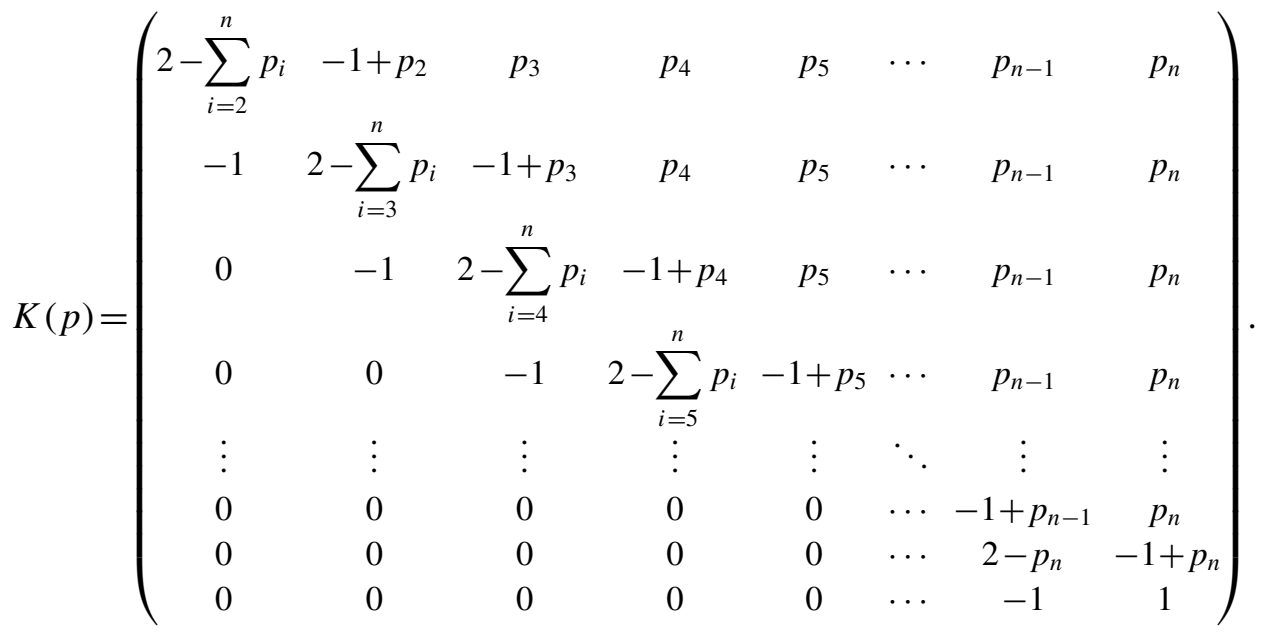

Its skew-symmetric part is

$$
K_{a}(p)=\frac{1}{2}\left(\begin{array}{cccccccc}
0 & p_{2} & p_{3} & p_{4} & p_{5} & \cdots & p_{n-1} & p_{n} \\
-p_{2} & 0 & p_{3} & p_{4} & p_{5} & \cdots & p_{n-1} & p_{n} \\
-p_{3} & -p_{3} & 0 & p_{4} & p_{5} & \cdots & p_{n-1} & p_{n} \\
-p_{4} & -p_{4} & -p_{4} & 0 & p_{5} & \cdots & p_{n-1} & p_{n} \\
\vdots & \vdots & \vdots & \vdots & \vdots & \ddots & \vdots & \vdots \\
-p_{n-1} & -p_{n-1} & -p_{n-1} & -p_{n-1} & -p_{n-1} & \cdots & 0 & p_{n} \\
-p_{n} & -p_{n} & -p_{n} & -p_{n} & -p_{n} & \cdots & -p_{n} & 0
\end{array}\right)
$$

and

$$
\operatorname{rank}\left(K_{a}(p)\right)= \begin{cases}n & \text { if } n \text { even } \\ n-1 & \text { if } n \text { odd }\end{cases}
$$

thus

$$
\ell= \begin{cases}n / 2 & \text { if } n \text { even, } \\ (n-1) / 2 & \text { if } n \text { odd }\end{cases}
$$

For $n=2$, calculations give

$$
K_{a}^{2}=-\frac{1}{4} p_{2}^{2}\left(\begin{array}{ll}
1 & 0 \\
0 & 1
\end{array}\right), \quad-\mu_{1}^{2}=-\frac{1}{4} p_{2}^{2} .
$$



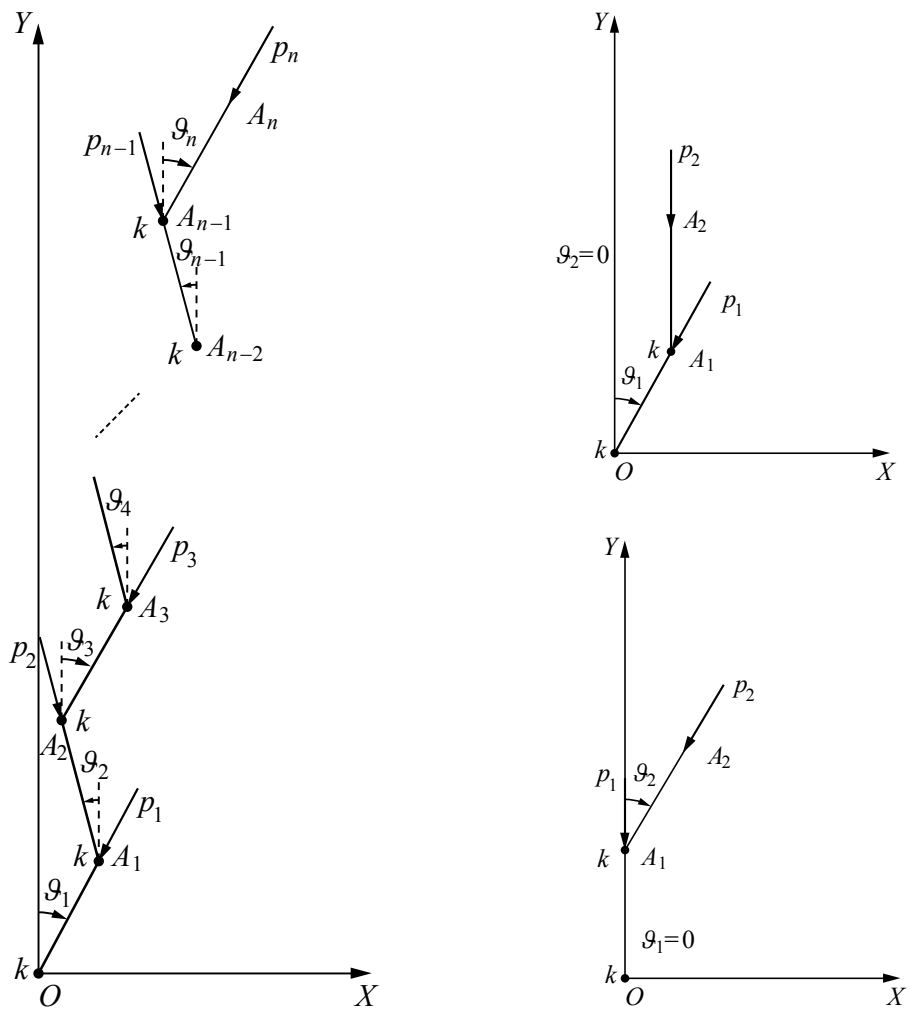

Figure 2. Bigoni systems with $n$ DOF (left) and two DOF (right).

$K_{a}^{2}$ is spherical, $E_{-\mu_{1}^{2}}\left(K_{a}^{2}\right)=\mathbb{R}^{2}$, and $\alpha$ is then any vector in $\mathbb{R}^{2}$. The geometric degree of nonconservativity is equal to 1 and the constraint is a linear combination of the two generic constraints $\theta_{1}=0$ and $\theta_{2}=0$ : this is any linear constraint! (See Figure 2, right.)

For $n=4$, calculations give

$$
-\mu_{1}^{2}=-\frac{3}{8} p_{4}^{2}-\frac{1}{4} p_{3}^{2}-\frac{1}{8} p_{2}^{2}+\frac{1}{8} a, \quad-\mu_{2}^{2}=-\frac{3}{8} p_{4}^{2}-\frac{1}{4} p_{3}^{2}-\frac{1}{8} p_{2}^{2}-\frac{1}{8} a,
$$

where $a=\sqrt{9 p_{4}^{4}+12 p_{3}^{2} p_{4}^{2}+2 p_{4}^{2} p_{2}^{2}+4 p_{3}^{4}+4 p_{3}^{2} p_{2}^{2}+p_{2}^{4}}$, and

$$
\begin{gathered}
E_{-\mu_{1}^{2}}\left(K_{a}^{2}\right)=\operatorname{Vec}\left\{\alpha_{1}=\left[\left[2 \left(-p_{3}^{2} p_{4}^{2} a+2 p_{3} p_{2} p_{4}^{2} a-p_{4}^{2} p_{2}^{2} a+p_{3} p_{2}^{3} a\right.\right.\right.\right. \\
+p_{3}^{3} p_{2} a-p_{4}^{2} p_{2}^{4}+3 p_{3} p_{2}^{3} p_{4}^{2}+5 p_{3}^{3} p_{2} p_{4}^{2}+2 p_{3} p_{2} p_{4}^{4}+p_{3}^{2} p_{2}^{2} p_{4}^{2}+p_{4}^{4} p_{2}^{2} \\
\left.\left.+7 p_{4}^{4} p_{3}^{2}+6 p_{4}^{6}+2 p_{4}^{2} p_{3}^{4}-2 p_{4}^{4} a+2 p_{3}^{5} p_{2}+3 p_{3}^{3} p_{2}^{3}+p_{3} p_{2}^{5}\right)\right], \\
{\left[-\left(2 p_{3}^{3} p_{2}+5 p_{3} p_{2} p_{4}^{2}+p_{3} p_{2}^{3}+3 p_{4}^{2} p_{2}^{2}+p_{3} p_{2} a+3 p_{4}^{4}\right.\right.} \\
\left.\left.+2 p_{3}^{2} p_{4}^{2}-p_{4}^{2} a\right)\left(-p_{4}^{2}+p_{2}^{2}+a\right)\right],\left[\left(-3 p_{4}^{4}-2 p_{3}^{2} p_{4}^{2}+2 p_{4}^{2} p_{2}^{2}\right.\right. \\
\left.\left.\left.\left.+p_{4}^{2} a+2 p_{3}^{2} p_{2}^{2}+p_{2}^{4}+p_{2}^{2} a\right)\left(-p_{4}^{2}+p_{2}^{2}+a\right)\right],[0]\right]\right\},
\end{gathered}
$$




$$
\begin{gathered}
E_{-\mu_{2}^{2}}\left(K_{a}^{2}\right)=\operatorname{Vec}\left\{\alpha_{2}=\left[\left[2 p _ { 4 } \left(p_{3}^{3} a+p_{2}^{3} a+p_{3}^{2} p_{2} a+p_{3} p_{2}^{2} a\right.\right.\right.\right. \\
+2 p_{4}^{2} p_{2} a+2 p_{4}^{2} p_{3} a+p_{3} p_{2}^{4}+2 p_{4}^{4} p_{2}+3 p_{4}^{2} p_{2}^{3}+3 p_{3}^{2} p_{2}^{3}-7 p_{3}^{3} p_{4}^{2} \\
\left.\left.-6 p_{3} p_{4}^{4}-2 p_{3}^{5}-p_{4}^{2} p_{2}^{2} p_{3}+5 p_{4}^{2} p_{3}^{2} p_{2}-p_{2}^{2} p_{3}^{3}+2 p_{3}^{4} p_{2}+p_{2}^{5}\right)\right], \\
{\left[-p_{4}\left(2 p_{3}^{2} p_{2}+5 p_{4}^{2} p_{2}+p_{2}^{3}-3 p_{3} p_{2}^{2}+p_{2} a-3 p_{4}^{2} p_{3}-2 p_{3}^{3}\right.\right.} \\
\left.\left.+p_{3} a\right)\left(-p_{4}^{2}+p_{2}^{2}+a\right)\right],[0],\left[\left(-3 p_{4}^{4}-2 p_{3}^{2} p_{4}^{2}\right.\right. \\
\left.\left.\left.\left.+2 p_{4}^{2} p_{2}^{2}+p_{4}^{2} a+2 p_{3}^{2} p_{2}^{2}+p_{2}^{4}+p_{2}^{2} a\right)\left(-p_{4}^{2}+p_{2}^{2}+a\right)\right]\right]\right\} .
\end{gathered}
$$

For $p_{i}=\frac{c}{i h}$, we have

$$
\begin{aligned}
-\mu_{1}^{2} & =\frac{c^{2}}{1152 h^{2}}(-95+\sqrt{7729}), \\
-\mu_{2}^{2} & =\frac{c^{2}}{1152 h^{2}}(-95-\sqrt{7729}), \\
a & =\frac{1}{144} \frac{\sqrt{7729} c^{2}}{h^{2}},
\end{aligned}
$$

so

$$
\begin{aligned}
& E_{-\mu_{1}^{2}}\left(K_{a}^{2}\right)=\operatorname{Vec}\left\{\alpha_{1}=\frac{c^{6}}{h^{6}}\left(\begin{array}{c}
\frac{35}{6} \frac{\sqrt{7729}+137}{27+\sqrt{7729}} \\
-\frac{1}{24}(281-\sqrt{7729}) \\
\frac{1}{8}(57+\sqrt{7729}) \\
0
\end{array}\right), K_{a} \alpha_{1}\right\}, \\
& E_{-\mu_{2}^{2}}\left(K_{a}^{2}\right)=\operatorname{Vec}\left\{\alpha_{2}=\frac{c^{6}}{h^{6}}\left(\begin{array}{c}
\frac{35}{3} \frac{\sqrt{7729}+37}{27+\sqrt{7729}} \\
-\frac{1}{12}(1-\sqrt{7729}) \\
0 \\
\frac{1}{8}(57+\sqrt{7729})
\end{array}\right), K_{a} \alpha_{2}\right\} .
\end{aligned}
$$

In this example, the geometric degree of nonconservativity is equal to 2: two additional kinematic constraints $\phi_{1}\left(\theta_{1}, \ldots, \theta_{4}\right)=0$ and $\phi_{2}\left(\theta_{1}, \ldots, \theta_{4}\right)=0$ are then necessary to convert the system into a conservative one, each constraint $\phi_{i}$ being chosen in $E_{-\mu_{i}^{2}}\left(K_{a}^{2}\right)$ for $i=1,2$. For example,

$$
\begin{aligned}
& \phi_{1}\left(\theta_{1}, \ldots, \theta_{4}\right)=\frac{35}{6} \frac{\sqrt{7729}+137}{27+\sqrt{7729}} \theta_{1}-\frac{1}{24}(281-\sqrt{7729}) \theta_{2}+\frac{1}{8}(57+\sqrt{7729}) \theta_{3}, \\
& \phi_{2}\left(\theta_{1}, \ldots, \theta_{4}\right)=\frac{35}{6} \frac{\sqrt{7729}+137}{27+\sqrt{7729}} \theta_{1}-\frac{1}{12}(1-\sqrt{7729}) \theta_{2}+\frac{1}{8}(57+\sqrt{7729}) \theta_{4} .
\end{aligned}
$$

\section{Conclusion}

In this paper, we investigate nonconservative systems, meaning here elastic systems with a nonsymmetric stiffness matrix. We associate with each mechanical system 
a minimal number $\ell$ of additional kinematic constraints allowing this system to be converted into a conservative one. As this integer measure of the nonconservativity of the mechanical system is linked with the dimension of a vector space, it is called the geometric degree of nonconservativity of the system. Computations of this integer and of the corresponding additional kinematic constraints are constructive and several examples illustrate the results. The extension to the nonlinear case will be developed in a forthcoming paper.

\section{References}

[Absi and Lerbet 2004] E. Absi and J. Lerbet, "Instability of elastic bodies", Mech. Res. Commun. 31 (2004), 39-44.

[Bigoni and Noselli 2011] D. Bigoni and G. Noselli, "Experimental evidence of flutter and divergence instabilities induced by dry friction”, J. Mech. Phys. Solids 59 (2011), 2208-2226.

[Bolotin 1961] V. V. Bolotin, Неконсервативные задачи теории упругой устойчивости, Fizmatlit, Moscow, 1961. Translated as Nonconservative problems of the theory of elastic stability, Macmillan, New York, 1963.

[Challamel et al. 2009] N. Challamel, F. Nicot, J. Lerbet, and F. Darve, "On the stability of nonconservative elastic systems under mixed perturbations”, Eur. J. Environ. Civ. Eng. 13:3 (2009), $347-367$.

[Challamel et al. 2010] N. Challamel, F. Nicot, J. Lerbet, and F. Darve, "Stability of non-conservative elastic structures under additional kinematic constraints", Eng. Struct. 32:10 (2010), 3086-3092.

[Hill 1958] R. Hill, "A general theory of uniqueness and stability in elastic-plastic solids", J. Mech. Phys. Solids 6:3 (1958), 236-249.

[Kirillov and Verhulst 2010] O. N. Kirillov and F. Verhulst, "Paradoxes of dissipation-induced destabilization or who opened Whitney's umbrella?", Z. Angew. Math. Mech. 90:6 (2010), 462-488.

[Leipholz 1987] H. Leipholz, Stability theory: an introduction to the stability of dynamic systems and rigid bodies, 2nd ed., Wiley, New York, 1987.

[Lerbet et al. 2012] J. Lerbet, M. Aldowaji, N. Challamel, F. Nicot, F. Prunier, and F. Darve, “Ppositive definite matrices and stability of nonconservative systems", Z. Angew. Math. Mech. 92:5 (2012), 409-422.

[Lerbet et al. 2013] J. Lerbet, O. Kirillov, M. Aldowaji, N. Challamel, F. Nicot, and F. Darve, "Additional constraints may soften a nonconservative structural system: buckling and vibration analysis", Int. J. Solids Struct. 50:2 (2013), 363-370.

[Nicot et al. 2011] F. Nicot, N. Challamel, J. Lerbet, F. Prunier, and F. Darve, "Bifurcation and generalized mixed loading conditions in geomaterials", Int. J. Numer. Anal. Methods Geomech. 35:13 (2011), 1409-1431.

[Zhuravlëv 2007] V. F. Zhuravlëv, “О разложении нелинейных обобщенных сил на потенциальную и циркулярную компоненты”, Dokl. Phys. 414:5 (2007), 622-624. Translated as "Decomposition of nonlinear generalized forces into potential and circulatory components" in Doklady Physics 52:6 (2007), 339-341.

[Ziegler 1952] H. Ziegler, "Die Stabilitätskriterien der Elastomechanik", Ing. Arch. 20 (1952), 4956.

Received 9 Nov 2012. Revised 15 Apr 2013. Accepted 25 May 2013. 
JEAN LERBET: jean.lerbet@ibisc.univ-evry.fr

Informatique Biologie Intégrative Systèmes Complexes, Université d'Evry-Val-d'Essonne, UFR Sciences \& Technologies, 40, Rue du Pelvoux, CE 1455 Courcouronnes, 91020 Evry cedex, France

MARWA ALDOWAJI: marwadoh@yahoo.com

Informatique Biologie Intégrative Systèmes Complexes, Université d'Evry-Val-d'Essonne, UFR Sciences \& Technologies, 40, Rue du Pelvoux, CE 1455 Courcouronnes, 91020 Evry cedex, France

NoËL CHALlamel: noel. challamel@univ-ubs.fr

Laboratoire d'Ingénierie des Matériaux de Bretagne, Université de Bretagne Sud, Rue de saint Maudé, BP 92116, 56321 Lorient cedex, France

OLEG N. KIRILLOV: o.kirillov@hzdr.de

Department of Magnetohydrodynamics, Institute of Fluid Dynamics, Helmholtz-Zentrum Dresden-Rossendorf, Bautzner Landstraße 400, P.O. Box 510119, 01314 Dresden, Germany

FRANÇOIS NICOT: Francois.Nicot@irstea.fr

Geomechanics Group, Irstea, Domaine Universitaire BP 76, 38402 Saint Martin d'Hères cedex, France

FÉLIX DARVE: felix.darve@3sr-grenoble.fr

Laboratoire Sols Solides Structures, UJF-INPG-CNRS, BP 53, 38041 Grenoble cedex 9, France 



\title{
ASYMPTOTIC ANALYSIS OF SMALL DEFECTS NEAR A SINGULAR POINT IN ANTIPLANE ELASTICITY, WITH AN APPLICATION TO THE NUCLEATION OF A CRACK AT A NOTCH
}

\author{
Thi BACH Tuyet DAng, LAURence Halpern And JeAn-JACQUes MARigo
}

\begin{abstract}
We use matching asymptotic expansions to treat the antiplane elastic problem associated with a small defect located at the tip of a notch. In a first part, we develop the asymptotic method for any type of defect and present the sequential procedure which allows us to calculate the different terms of the inner and outer expansions at any order. This requires in particular separating in each term its singular part from its regular part. In a second part, the asymptotic method is applied to the case of a crack of variable length located at the tip of a given notch. We show that the first two nontrivial terms of the expansion of the energy release rate are sufficient to well approximate the dependence of the energy release rate on the crack length in the range of values of the length which are sufficient to treat the problem of nucleation. This problem is considered in the last part where we compare the nucleation and the propagation of a crack predicted by two different models: the classical Griffith law and the Francfort-Marigo law based on an energy minimization principle. Several numerical results illustrate the interest of the method.
\end{abstract}

\section{Introduction}

A major issue in fracture mechanics is how to model the initiation of a crack in a sound material; see [Bourdin et al. 2008]. There are two difficulties: the first one is to propose a law able to predict that nucleation; the second is a purely numerical issue. Indeed, it is difficult to compute with good accuracy the energy release rate associated with a crack of small length which appears at the tip of a notch; see [Marigo 2010]. The classical finite element method (FEM) leads to inaccurate

\section{Communicated by Francesco dell'Isola.}

This work was partially supported by the French Agence Nationale de la Recherche (ANR), under grant epsilon (BLAN08-2_312370) "Domain decomposition and multi-scale computations of singularities in mechanical structures".

MSC2000: 35A15, 35B40, 35C20, 74A45, 74G70, 74R10.

Keywords: brittle fracture, variational methods, asymptotic methods, singularities. 
results because of the overlap of two singularities which cannot be correctly captured by this method: one is due to the tip of the notch; the other is due to the tip of the crack. A specific method of approximation based on asymptotic expansions is preferable as it is developed in analogous situations with localized defects; see for instance [Abdelmoula and Marigo 2000; Abdelmoula et al. 2010; Bilteryst and Marigo 2003; Bonnaillie-Noël et al. 2010; 2011; David et al. 2012; Geymonat et al. 2011; Leguillon 1989; Marigo and Pideri 2011; Vidrascu et al. 2012]. The first part of the present paper is devoted to the presentation of this matched asymptotic method (shortly, the MAM) in the case of a defect (which includes the case of a crack) located at the tip of a notch in the simplified context of antiplane linear elasticity. Therefore, our approach can be considered as a particular case of the previous works which have been devoted to the study of elliptic problems in corner domains, like [Dauge 1988; Dauge et al. 2010; Grisvard 1985; 1986]. However, a major difference is that we want to use these asymptotic methods to predict the nucleation or the propagation of defects (like cracks) near those singular points. The second and third parts of our paper will be devoted to this task. This requires, of course, to introduce a criterion of nucleation. This delicate issue has not received a definitive answer at the present time and it was considered for a long time as a problem which could not be solved in the framework of Griffith's theory of fracture [Bui 1978; Cherepanov 1979; Lawn 1993; Leblond 2003]. The main invoked reason is that the release of energy due to a small crack tends to zero when the length of the crack tends to zero; see [Chambolle et al. 2008; Marigo 2010]. Therefore, according to the Griffith criterion which states that the crack can propagate only when the energy release rate reaches a critical value characteristic of the material, no nucleation is possible because the energy release rate vanishes when there is no preexisting crack. This limitation of Griffith's theory was one of the motivations which led Francfort and Marigo [1998] to replace the Griffith criterion by a principle of least energy, in the spirit of the original idea of [Griffith 1921]. It turns out that the principle of least energy is really able to predict the nucleation of cracks in a sound body. However, as it was generically proved in [Chambolle et al. 2008; Francfort and Marigo 1998], the nucleation is necessarily brutal in the sense that a crack of finite length suddenly appears at a critical loading. Accordingly, we propose to revisit the problem of nucleation of a crack at the tip of a notch by comparing the two criteria. One of our goals is to use the MAM to obtain semianalytical expressions for the critical loading at which a crack appears and the length of the nucleated crack.

Specifically, the paper is organized as follows. Section 2 is devoted to the description of the MAM on a generic antiplane linear elastic problem where the body contains a defect near the tip of a notch. We first decompose the solution into two expansions: the outer expansion is valid far enough from the tip of the notch while the inner expansion is valid in a neighborhood of the tip of the notch. These 
expansions contain a sequence of inner and outer terms which are solutions of inner and outer problems and are connected by the matching conditions. Moreover each term contains a regular and a singular part. We explain how all the terms and the coefficients entering in their singular and regular parts are sequentially determined. The section finishes with an example where the exact solution is obtained in closed form and hence where we can verify the relevance of the MAM.

In Section 3, the MAM is applied to the case where the defect is a crack. Its main goal is to compute with good accuracy the energy release rate associated with a crack of small length near the tip of the notch. Indeed, it is a real issue in the case of a genuine notch (as opposed to a crack) because the energy release rate starts from 0 when the length of the nucleated crack is 0 , then is rapidly increasing with the length of the crack before reaching a maximum and is finally decreasing. Accordingly, after the setting of the problem, the computation of the energy release rate by the FEM is described, and the reason why the numerical results are less accurate when the crack length is small is given. Then, the MAM is used to compute the energy release rate for small values of the crack length. As expected, the computation shows that, the smaller the size of the defect, the more accurate is the approximation by the MAM at a certain order. It even appears that very accurate results can be obtained by computing a small number of terms in the matched asymptotic expansions. We discuss also the influence of the angle of the notch on the accuracy of the results, this angle playing an important role in the process of nucleation (because, in particular, the length $l_{\mathrm{m}}$ at which the maximum of the energy release rate is reached depends on the angle of the notch). It turns out that when the notch is sufficiently sharp, i.e., sufficiently close to a crack, the first two nontrivial terms of the expansion of the energy release rate are sufficient to capture with very good accuracy the dependence of the energy release rate on the crack length.

In Section 4, we study the problem of crack nucleation at the tip of a notch. We first introduce the two competing evolution laws, i.e., the G-law and the FM-law: the first one is the usual Griffith's law based on the criterion of critical energy release rate; the second is that introduced in [Francfort and Marigo 1998], which is based on the concept of energy minimization. We recall some general results previously established in [Marigo 2010] and extend them to the present case of a notch-shaped body in an antiplane setting. By virtue of the good approximation given by the MAM, we are able to solve the evolution problem in a quasiclosed form, the solution depending only on two coefficients that must be computed by the FEM. This permits a qualitative and quantitative comparison of the two laws. 


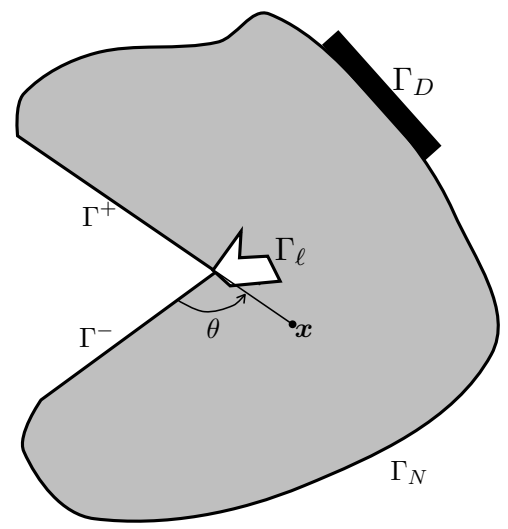

Figure 1. The domain $\Omega_{l}$ for the real problem.
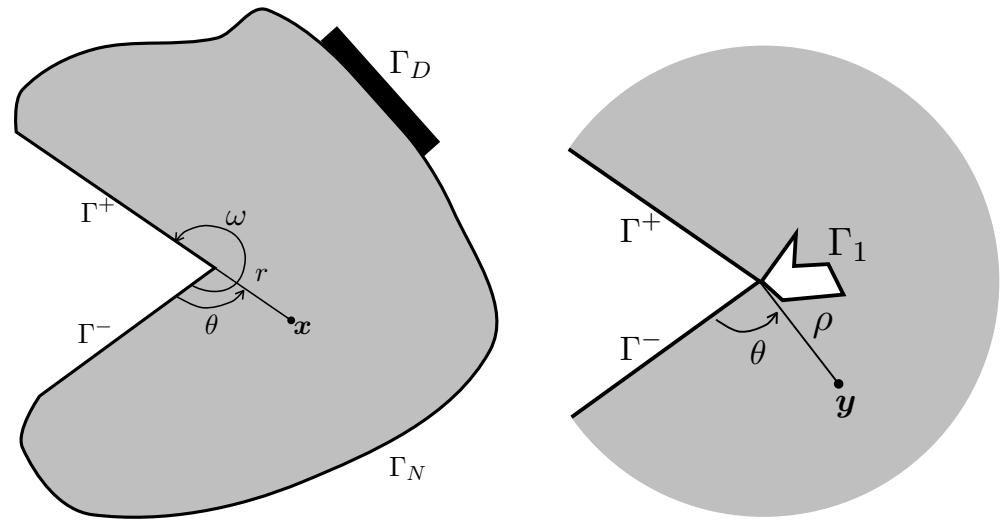

Figure 2. The domains $\Omega_{0}$ and $\Omega^{\infty}$ for, respectively, the outer (left) and the inner (right) problems.

\section{The real problem and its expansion by the matched asymptotic method}

2.1. The real problem. Here, we consider a small geometrical defect of size $l$ (like a crack or a void) located near the corner of a notch; see Figure 1. The geometry of the notch is characterized by its angle $\omega$; see Figure 2. The tip of the notch is taken as the origin of the space. We will introduce two scales of coordinates: the "macroscopic" coordinates $\boldsymbol{x}=\left(x_{1}, x_{2}\right)$ used in the outer domain, and the "microscopic" coordinates $\boldsymbol{y}=\boldsymbol{x} / l=\left(y_{1}, y_{2}\right)$ used in the neighborhood of the tip of the notch where the defect is located; see Figure 2. In the case of a crack, the axis $x_{1}$ is chosen in such a way that the crack corresponds to the line segment $(0, l) \times\{0\}$. The unit vector orthogonal to the $\left(x_{1}, x_{2}\right)$ plane is denoted by $\boldsymbol{e}_{3}$. 
The natural reference configuration of the sound two-dimensional body is $\Omega_{0}$, while the associated body which contains a defect of size $l$ is $\Omega_{l}$. The part of the boundary of $\Omega_{l}$ which is due to the defect is denoted by $\Gamma_{l}$; i.e.,

$$
\Gamma_{l}=\partial \Omega_{l} \backslash \partial \Omega_{0},
$$

and $\Gamma_{l}$ is contained in the disk of center $(0,0)$ and radius $l$. In the case of a crack, $\Gamma_{l}$ is the crack itself; i.e., $\Gamma_{l}=(0, l) \times\{0\}$. The two edges of the notch are denoted by $\Gamma^{+}$and $\Gamma^{-}$. To simplify the presentation, it is assumed that they are not modified by the introduction of the defect; see Figure 1 . When using polar coordinates $(r, \theta)$, the pole is the tip of the notch and the origin of the polar angle is the edge $\Gamma^{-}$. Accordingly, we have

$$
r=|x|, \quad \Gamma^{-}=\left\{(r, \theta): 0<r<r^{*}, \theta=0\right\}, \quad \Gamma^{+}=\left\{(r, \theta): 0<r<r^{*}, \theta=\omega\right\} .
$$

This body is made of an elastic isotropic material whose shear modulus is $\mu>0$. It is submitted to a loading such that the displacement field at equilibrium $\boldsymbol{u}_{l}$ be antiplane; i.e.,

$$
\boldsymbol{u}_{l}(\boldsymbol{x})=u_{l}\left(x_{1}, x_{2}\right) \boldsymbol{e}_{3},
$$

where the subscript letter $l$ is used as a reminder that the real displacement depends on the size of the defect. We assume that the body forces are zero and then $u_{l}$ must be an harmonic function in order to satisfy the equilibrium equations in the bulk:

$$
\Delta u_{l}=0 \quad \text { in } \Omega_{l} .
$$

The edges of the notch are free while $\Gamma_{l}$ is submitted to a density of (antiplane) surface forces. Accordingly, the boundary conditions on $\Gamma_{l}$ and $\Gamma^{ \pm}$are

$$
\frac{\partial u_{l}}{\partial v}=0 \quad \text { on } \Gamma^{ \pm}, \quad \frac{\partial u_{l}}{\partial v}(\boldsymbol{x})=\frac{g(\boldsymbol{y})}{l} \quad \text { on } \Gamma_{l} .
$$

In (4), $v$ denotes the unit outer normal vector to $\Omega_{l}$, and we assume that the density of (antiplane) surface forces depends on the microscopic variable $\boldsymbol{y}$ and has a magnitude of the order of $1 / l$.

The remaining part of the boundary of $\Omega_{l}$ is divided into two parts: $\Gamma_{D}$ where the displacement is prescribed and $\Gamma_{N}$ where (antiplane) surface forces are prescribed. Specifically, we have

$$
u_{l}=f \quad \text { on } \Gamma_{D}, \quad \frac{\partial u_{l}}{\partial v}=h \quad \text { on } \Gamma_{N} .
$$

The following proposition is a characterization of those functions which are harmonic in an angular sector and whose normal derivatives vanish on the edges of the sector. It is of constant use throughout the paper. 
Proposition 1. Let $r_{1}$ and $r_{2}$ be such that $0 \leq r_{1}<r_{2} \leq+\infty$ and let $\mathscr{D}_{r_{1}}^{r_{2}}$ be the angular sector

$$
\mathscr{D}_{r_{1}}^{r_{2}}=\left\{(r, \theta): r \in\left(r_{1}, r_{2}\right), \theta \in(0, \omega)\right\} .
$$

Then any function $u$ which is harmonic in $\mathscr{D}_{r_{1}}^{r_{2}}$ and which satisfies the Neumann condition $\partial u / \partial \theta=0$ on the sides $\theta=0$ and $\theta=\omega$ can be expanded as

$$
u(r, \theta)=\mathrm{a}_{0} \ln (r)+\mathrm{d}_{0}+\sum_{n \in \mathbb{N}^{*}}\left(\mathrm{a}_{n} r^{-n \lambda}+\mathrm{d}_{n} r^{n \lambda}\right) \cos (n \lambda \theta), \quad \lambda=\frac{\pi}{\omega},
$$

where the $\mathrm{a}_{n}$ and the $\mathrm{d}_{n}$ constitute two sequences of real numbers which are characteristic of $u$.

Proof. Since the normal derivative vanishes at $\theta=0$ and $\theta=\omega, u(r, \theta)$ can be expanded in a Fourier series as

$$
u(r, \theta)=\sum_{n \in \mathbb{N}} f_{n}(r) \cos (n \lambda \theta) .
$$

In order that $u$ be harmonic, the functions $f_{n}$ must satisfy $r^{2} f_{n}^{\prime \prime}+r f_{n}^{\prime}-n^{2} \lambda^{2} f_{n}=0$ for each $n$. We easily deduce that $f_{0}(r)=\mathrm{a}_{0} \ln (r)+\mathrm{d}_{0}$ and $f_{n}(r)=\mathrm{a}_{n} r^{-n \lambda}+\mathrm{d}_{n} r^{n \lambda}$ for $n \geq 1$.

2.2. The matching asymptotic method (MAM). We will write two asymptotic expansions of $u_{l}$ in terms of the small parameter $l$. The inner expansion is valid in the neighborhood of the tip of the notch, while the outer expansion is valid far from this tip. These two expansions will be matched in an intermediate zone.

2.2.1. The outer expansion. Far from the tip of the notch, i.e., for $r \gg l, u_{l}$ does not see the notch, and we assume that it can be expanded as

$$
u_{l}(\boldsymbol{x})=\sum_{i \in \mathbb{N}} l^{i \lambda} u^{i}(\boldsymbol{x})
$$

In (7), even if this expansion is valid far enough from $r=0$ only, $u^{i}$ must be defined in the whole outer domain $\Omega_{0}$ which corresponds to the sound body; see Figure 2 (left). Inserting this expansion into (3), (4), and (5) yields the sequence of problems for the $u^{i}$ :

The first outer problem, $i=0$ :

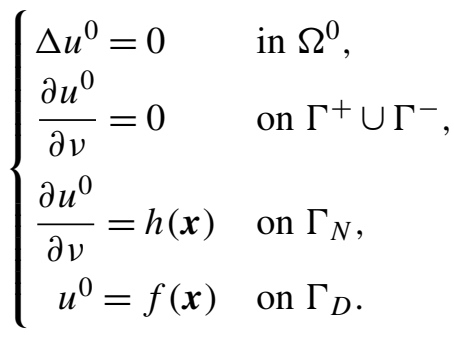


The other outer problems, $i \geq 1$ :

$$
\begin{cases}\Delta u^{i}=0 & \text { in } \Omega^{0}, \\ \frac{\partial u^{i}}{\partial v}=0 & \text { on } \Gamma^{+} \cup \Gamma^{-}, \\ \frac{\partial u^{i}}{\partial v}=0 & \text { on } \Gamma_{N}, \\ u^{i}=0 & \text { on } \Gamma_{D} .\end{cases}
$$

Moreover, the behavior of $u^{i}$ in the neighborhood of $r=0$ is singular and the singularity will be given by the matching conditions.

2.2.2. The inner expansion. Near the tip of the notch, i.e., for $r \ll 1$, we assume that the displacement field $u_{l}$ can be expanded as

$$
u_{l}(\boldsymbol{x})=\ln (l) \sum_{i \in \mathbb{N}} l^{i \lambda} w^{i}(\boldsymbol{y})+\sum_{i \in \mathbb{N}} l^{i \lambda} v^{i}(\boldsymbol{y}), \quad \boldsymbol{y}=\frac{\boldsymbol{x}}{l} .
$$

In (10), even if this expansion is valid only in the neighborhood of $r=0$, the fields $v^{i}$ and $w^{i}$ must be defined in the infinite inner domain $\Omega^{\infty}$. The domain $\Omega^{\infty}$ is the infinite angular sector $\mathscr{D}_{0}^{\infty}$ of the $\left(y_{1}, y_{2}\right)$ plane, from which the rescaled defect of size 1 is removed; see Figure 2 (right). Accordingly, the rescaled boundary $\Gamma_{1}$ of the defect is

$$
\Gamma_{1}=\partial \Omega^{\infty} \backslash \partial \mathscr{D}_{0}^{\infty} .
$$

(In the case of a crack, $\Gamma_{1}=(0,1) \times\{0\}$.) Inserting this expansion into the set of equations constituting the real problem yields the sequence of problems for the $v^{i}$ :

The first inner problem, $i=0$ :

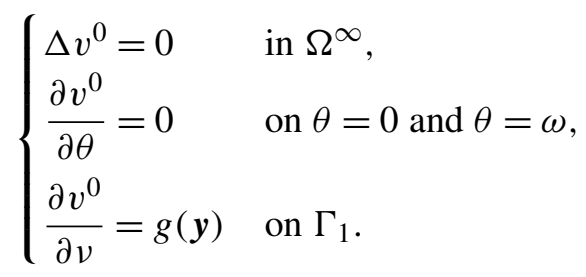

The other inner problems, $i \geq 1$ :

$$
\begin{cases}\Delta v^{i}=0 & \text { in } \Omega^{\infty}, \\ \frac{\partial v^{i}}{\partial \theta}=0 & \text { on } \theta=0 \text { and } \theta=\omega, \\ \frac{\partial v^{i}}{\partial v}=0 & \text { on } \Gamma_{1} .\end{cases}
$$


The $w^{i}$ must satisfy, for every $i \geq 0$, the same equations as the $v^{i}$ for $i \geq 1$. To complement the set of equations, the behavior at infinity of the $v^{i}$ and the $w^{i}$ must be included. It is obtained by the matching conditions from the outer problems.

2.2.3. Matching conditions. In any sector $\mathscr{D}_{0}^{r_{2}}$ with $l \ll r_{2} \ll 1$, the displacement fields $u^{i}$ in the outer expansion are harmonic and satisfy homogeneous Neumann boundary conditions on the edges. Therefore Proposition 1 applies, and

$$
u^{i}(\boldsymbol{x})=\mathrm{a}_{0}^{i} \ln (r)+\mathrm{d}_{0}^{i}+\sum_{n \in \mathbb{N}^{*}}\left(\mathrm{a}_{n}^{i} r^{-n \lambda}+\mathrm{d}_{n}^{i} r^{n \lambda}\right) \cos (n \lambda \theta) .
$$

As for the inner expansion, the displacement fields $v^{i}$ and $w^{i}$ are harmonic in the sector $\mathscr{D}_{1}^{\infty}$ of the $\boldsymbol{y}$ plane and satisfy homogeneous Neumann boundary conditions on the edges. Therefore Proposition 1 applies, with the microscopic coordinates $y$ and $\rho=|\boldsymbol{y}|=r / l$ replacing the macroscopic coordinates $\boldsymbol{x}$ and $r$ :

$$
\begin{aligned}
& v^{i}(\boldsymbol{y})=\mathrm{c}_{0}^{i} \ln (\rho)+\mathrm{b}_{0}^{i}+\sum_{n \in \mathbb{N}^{*}}\left(\mathrm{c}_{n}^{i} \rho^{-n \lambda}+\mathrm{b}_{n}^{i} \rho^{n \lambda}\right) \cos (n \lambda \theta), \\
& w^{i}(\boldsymbol{y})=\mathrm{e}_{0}^{i} \ln (\rho)+\mathrm{f}_{0}^{i}+\sum_{n \in \mathbb{N}^{*}}\left(\mathrm{e}_{n}^{i} \rho^{-n \lambda}+\mathrm{f}_{n}^{i} \rho^{n \lambda}\right) \cos (n \lambda \theta) .
\end{aligned}
$$

The outer expansion and the inner expansion are both valid in any intermediate zone $\mathscr{D}_{r_{1}}^{r_{2}}$ such that $l \ll r_{1}<r_{2} \ll 1$. Inserting (14) into the outer expansion (7) with $r=l \rho$ leads to

$$
\begin{aligned}
u_{l}(\boldsymbol{x})= & \sum_{i \in \mathbb{N}} \ln (l) l^{i \lambda} \mathrm{a}_{0}^{i} \\
& +\sum_{i \in \mathbb{N}} l^{i \lambda}\left(\mathrm{a}_{0}^{i} \ln (\rho)+\mathrm{d}_{0}^{i}+\sum_{n \in \mathbb{N}^{*}}\left(\mathrm{a}_{n}^{i+n} \rho^{-n \lambda}+\mathrm{d}_{n}^{i-n} \rho^{n \lambda}\right) \cos (n \lambda \theta)\right),
\end{aligned}
$$

with the convention that $\mathrm{d}_{n}^{i-n}=0$ when $n>i$. Inserting (15) and (16) into the inner expansion (10) leads to

$$
\begin{aligned}
u_{l}(\boldsymbol{x})=\sum_{i \in \mathbb{N}} \ln (l) l^{i \lambda}\left(\mathrm{e}_{0}^{i} \ln (\rho)+\mathrm{f}_{0}^{i}+\sum_{n \in \mathbb{N}^{*}}\left(\mathrm{e}_{n}^{i} \rho^{-n \lambda}+\mathrm{f}_{n}^{i} \rho^{n \lambda}\right) \cos (n \lambda \theta)\right) \\
+\sum_{i \in \mathbb{N}} l^{i \lambda}\left(\mathrm{c}_{0}^{i} \ln (\rho)+\mathrm{b}_{0}^{i}+\sum_{n \in \mathbb{N}^{*}}\left(\mathrm{c}_{n}^{i} \rho^{-n \lambda}+\mathrm{b}_{n}^{i} \rho^{n \lambda}\right) \cos (n \lambda \theta)\right) .
\end{aligned}
$$

Both expansions (17) and (18) are valid provided that $1 \ll \rho \ll 1 / l$. Identification of these expansions provides the connections between the coefficients of the inner and outer expansions described in Table 1.

Remark 1. From Table 1 can be deduced that the fields $w^{i}$ are constant in the whole inner domain:

$$
w^{i}(\boldsymbol{y})=\mathrm{a}_{0}^{i} \quad \text { for all } \boldsymbol{y} \in \Omega^{\infty} \text { and all } i \geq 0 .
$$




$$
\begin{array}{|lc}
\mathrm{e}_{n}^{i}=0 & i \geq 0, n \geq 0 \\
\mathrm{f}_{0}^{i}=\mathrm{a}_{0}^{i} & i \geq 0 \\
\mathrm{f}_{n}^{i}=0 & i \geq 0, n \geq 1 \\
\mathrm{a}_{n}^{i}=0 & n>i \geq 0 \\
\mathrm{c}_{n}^{i}=\mathrm{a}_{n}^{i+n} & i \geq 0, n \geq 0 \\
\mathrm{~b}_{n}^{i}=0 & n>i \geq 0 \\
\mathrm{~d}_{n}^{i}=\mathrm{b}_{n}^{i+n} & i \geq 0, n \geq 0
\end{array}
$$

Table 1. The relations between the coefficients of the inner and outer expansions given by the matching conditions.

Therefore, these fields will be determined once the constants $a_{0}^{i}$ are known.

2.2.4. The singular behavior of the $u^{i}$ and the $v^{i}$. From the matching conditions can be read the behavior of $u^{i}$ in the neighborhood of $r=0$ and the behavior of $v^{i}$ at infinity. In particular, the form of their singularities is visible, according to the following definition.

Definition 1. A field $u$ defined in $\Omega_{0}$ is regular in $\Omega_{0}$ if $u \in H^{1}\left(\Omega_{0}\right)$; i.e., $u \in$ $L^{2}\left(\Omega_{0}\right)$ and $\nabla u \in L^{2}\left(\Omega_{0}\right)^{2}$. It is singular otherwise.

A field $u$ defined in the unbounded domain $\Omega^{\infty}$ is regular in $\Omega^{\infty}$ if $\nabla u \in$ $\left(L^{2}\left(\Omega^{\infty}\right)\right)^{2}$ and $\lim _{\rho \rightarrow \infty} u(\rho, \theta)=0$. It is singular otherwise.

Remark 2. In other words, a field is regular if the associated elastic energy is finite. It is singular otherwise. In the case of the unbounded domain $\Omega^{\infty}$, a constant field has finite energy, but the condition at infinity is added in order to fix the constant and obtain the uniqueness in the forthcoming boundary value problems.

According to the analysis in the previous subsection, the field $u^{0}$ can be expanded in a neighborhood of the tip of the notch as

$$
u^{0}(\boldsymbol{x})=\mathrm{a}_{0}^{0} \ln (r)+\sum_{n \in \mathbb{N}} \mathrm{b}_{n}^{n} r^{n \lambda} \cos (n \lambda \theta)
$$

In the domain $\Omega_{0}, \ln (r)$ is singular, whereas $r^{n \lambda} \cos (n \lambda \theta)$ is regular for $n \geq 0$, in the sense of Definition 1. Accordingly, $u^{0}$ is split into its singular and regular parts as follows:

$$
\begin{gathered}
u^{0}(\boldsymbol{x})=u_{S}^{0}(\boldsymbol{x})+\bar{u}^{0}(\boldsymbol{x}), \\
u_{S}^{0}(\boldsymbol{x})=\mathrm{a}_{0}^{0} \ln (r), \quad \bar{u}^{0} \in H^{1}\left(\Omega_{0}\right) .
\end{gathered}
$$


In the same way, for $i \geq 1$, the field $u^{i}$ can be expanded in a neighborhood of the tip of the notch as

$$
u^{i}(\boldsymbol{x})=\mathrm{a}_{0}^{i} \ln (r)+\sum_{n=1}^{i} \mathrm{a}_{n}^{i} r^{-n \lambda} \cos (n \lambda \theta)+\sum_{n \in \mathbb{N}} \mathrm{b}_{n}^{i+n} r^{n \lambda} \cos (n \lambda \theta)
$$

Since $r^{-n \lambda} \cos (n \lambda \theta)$ is singular (for $n \geq 0$ ) in the sense of Definition $1, u^{i}$ is split into its singular and regular parts as follows:

$$
\begin{gathered}
u^{i}(\boldsymbol{x})=u_{S}^{i}(\boldsymbol{x})+\bar{u}^{i}(\boldsymbol{x}), \\
u_{S}^{i}(\boldsymbol{x})=\mathrm{a}_{0}^{i} \ln (r)+\sum_{n=1}^{i} \mathrm{a}_{n}^{i} r^{-n \lambda} \cos (n \lambda \theta), \quad \bar{u}^{i} \in H^{1}\left(\Omega_{0}\right) .
\end{gathered}
$$

For the fields $v^{i}$ of the inner expansion, the behavior at infinity comes into play. By virtue of the analysis in the previous subsection, the field $v^{i}$ for $i \geq 0$ can be expanded for large $\rho$ as

$$
v^{i}(\boldsymbol{y})=\mathrm{a}_{0}^{i} \ln (\rho)+\sum_{n=0}^{i} \mathrm{~b}_{n}^{i} \rho^{n \lambda} \cos (n \lambda \theta)+\sum_{n \in \mathbb{N}^{*}} \mathrm{a}_{n}^{i+n} \rho^{-n \lambda} \cos (n \lambda \theta) .
$$

The field $\ln (\rho)$ as well as the fields $\rho^{n \lambda} \cos (n \lambda \theta)$, for $n \geq 0$, are singular in $\Omega^{\infty}$ in the sense of Definition 1 (even the constant field 1 corresponding to $n=0$ is singular). Since the fields $\rho^{-n \lambda} \cos (n \lambda \theta)$ are regular when $n \geq 1, v^{i}$ is split into its singular and regular parts as follows:

$$
\begin{gathered}
v^{i}(\boldsymbol{y})=v_{S}^{i}(\boldsymbol{y})+\bar{v}^{i}(\boldsymbol{y}), \\
v_{S}^{i}(\boldsymbol{y})=\mathrm{a}_{0}^{i} \ln (\rho)+\sum_{n=0}^{i} \mathrm{~b}_{n}^{i} \rho^{n \lambda} \cos (n \lambda \theta), \quad \nabla \bar{v}^{i} \in L^{2}\left(\Omega^{\infty}\right), \quad \lim _{|\boldsymbol{y}| \rightarrow \infty} \bar{v}^{i}(\boldsymbol{y})=0 .
\end{gathered}
$$

Remark 3. This analysis of the singularities shows that the singular parts of the fields $u^{i}$ and $v^{i}$ will be known once the coefficients $\mathrm{a}_{n}^{i}$ and $\mathrm{b}_{n}^{i}$ are determined for $0 \leq n \leq i$.

2.2.5. The problems defining the regular parts $\bar{u}^{i}$ and $\bar{v}^{i}$. The singular parts $\left(u_{S}^{i}, v_{S}^{i}\right)$ are harmonic and satisfy the homogeneous Neumann boundary conditions on the edges of the notch. Therefore the regular parts are harmonic too, with data expressed in terms of the singular fields. 
The first outer problem, $i=0$ : Find $\bar{u}^{0}$ regular in $\Omega_{0}$ such that

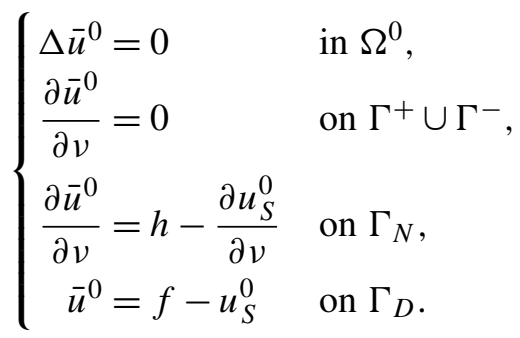

The other outer problems, $i \geq 1$ : Find $\bar{u}^{i}$ regular in $\Omega_{0}$ such that

$$
\left\{\begin{aligned}
\Delta \bar{u}^{i} & =0 & & \text { in } \Omega^{0}, \\
\frac{\partial \bar{u}^{i}}{\partial v} & =0 & & \text { on } \Gamma^{+} \cup \Gamma^{-}, \\
\frac{\partial \bar{u}^{i}}{\partial v} & =-\frac{\partial u_{S}^{i}}{\partial v} & & \text { on } \Gamma_{N}, \\
\bar{u}^{i} & =-u_{S}^{i} & & \text { on } \Gamma_{D} .
\end{aligned}\right.
$$

The first inner problem, $i=0$ : Find $\bar{v}^{0}$ regular in $\Omega^{\infty}$ such that

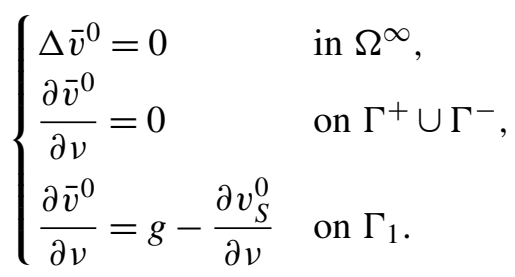

The other inner problems, $i \geq 1$ : Find $\bar{v}^{i}$ regular in $\Omega^{\infty}$ such that

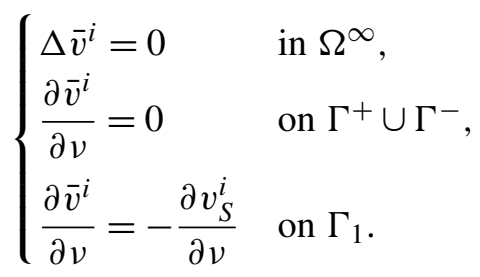

Consider first the outer problems. The well-posedness is a direct consequence of classical results for the Laplace equation:

Proposition 2. Let $i \geq 0$. For a given singular part $u_{S}^{i}$, i.e., if the coefficients $a_{n}^{i}$ are known for all $n$ such that $0 \leq n \leq i$, then there exists a unique solution $\bar{u}^{i}$ of (30) (or of (29) when $i=0)$. Consequently, the coefficients $\mathrm{b}_{n}^{i+n}$ are then determined for all $n \geq 0$. 
As for the inner problems, since they are Neumann problems (except for the condition at infinity), defined in an infinite domain, more care must be taken. The well-posedness is ensured by a compatibility condition, as stated in Proposition 3.

Proposition 3. Let $i \geq 0$. For given $\mathrm{b}_{n}^{i}$ with $0 \leq n \leq i$, there exists a regular solution $\bar{v}^{i}$ for the $i$-th inner problem if and only if the coefficient $\mathrm{a}_{0}^{i}$ is such that

$$
\mathrm{a}_{0}^{0}=-\frac{1}{\omega} \int_{\Gamma_{1}} g(s) d s, \quad \mathrm{a}_{0}^{i}=0 \quad \text { for } i \geq 1 .
$$

Moreover, if this condition is satisfied, then the solution is unique and therefore the coefficients $\mathrm{a}_{n}^{i+n}$ are determined for all $n \geq 0$.

Proof. The inner problems are pure Neumann problems in which no Dirichlet boundary conditions are imposed on the $v^{i}$ except for the condition at infinity. Consequently, they admit a solution (if and) only if the Neumann data satisfy a global compatibility condition. Let us reestablish that condition. Let $\Omega^{R}$ be the part of $\Omega^{\infty}$ included in the ball of radius $R>1$; i.e., $\Omega^{R}=\Omega^{\infty} \cap\{\boldsymbol{y}:|\boldsymbol{y}|<R\}$. Consider first the case $i=0$. Integrating the equation $\Delta v^{0}=0$ over $\Omega^{R}$ and using the boundary conditions leads to

$$
0=\int_{\partial \Omega^{R}} \frac{\partial v^{0}}{\partial v} d s=\int_{0}^{\omega} \frac{\partial v^{0}}{\partial \rho}(R, \theta) R d \theta+\int_{\Gamma_{1}} g(s) d s .
$$

Using (26) yields

$$
R \frac{\partial v^{0}}{\partial \rho}(R, \theta)=\mathrm{a}_{0}^{0}+\sum_{n \in \mathbb{N}^{*}} n \lambda\left(-\mathrm{c}_{n}^{0} R^{-n \lambda}+\mathrm{b}_{n}^{0} R^{n \lambda}\right) \cos (n \lambda \theta) .
$$

Since $\int_{0}^{\omega} \cos (n \lambda \theta) d \theta=0$ for all $n \geq 1$, after inserting in (34), the desired condition for $\mathrm{a}_{0}^{0}$ appears. For $i \geq 1$, the same process is applied, and the integral over $\Gamma_{1}$ vanishes, yielding the desired condition.

If the compatibility condition (33) is satisfied, then the existence of a regular solution for $\bar{v}^{i}$ is obtained by standard arguments. Note however that, since $\nabla \bar{v}^{i}$ belongs to $L^{2}\left(\Omega^{\infty}\right), \bar{v}^{i}$ tends to a constant at infinity and this constant is fixed to 0 by the additional regularity condition. As far as the uniqueness is concerned, the solution of this pure Neumann problem is unique up to a constant and the constant is fixed by the condition that $\bar{v}^{i}$ vanishes at infinity.

Once $v^{i}$ is determined, the coefficients $\mathrm{a}_{n}^{i+n}$ are obtained by virtue of Proposition 1 and (26).

Remark 4. If the forces applied to the boundary of the defect are equilibrated, i.e., if $\int_{\Gamma_{1}} g(s) d s=0$, then all the coefficients $a_{0}^{i}$ vanish and hence the terms in $\ln (l)$ disappear in the inner expansion. There are no more logarithmic singularities in the $u^{i}$ and the $v^{i}$. 
2.2.6. The construction of the outer and inner expansions. Recall the relationship between the coefficients $\left(\mathrm{a}_{n}^{j}, \mathrm{~b}_{n}^{j}\right)$ and the singular and regular parts of the $u_{j}$ and $v_{j}$ :

$$
\begin{array}{lll}
u^{j}=u_{S}^{j}+\bar{u}^{j}, & u_{S}^{j} \longleftrightarrow\left(\mathrm{a}_{n}^{j}\right)_{n=0}^{j}, & \bar{u}^{j} \longleftrightarrow\left(\mathrm{b}_{n}^{j+n}\right)_{n \geq 0}, \\
v^{j}=v_{S}^{j}+\bar{v}^{j}, & v_{S}^{j} \longleftrightarrow\left(\mathrm{a}_{0}^{j},\left(\mathrm{~b}_{n}^{j}\right)_{n=0}^{j}\right), & \bar{v}^{j} \longleftrightarrow\left(\mathrm{a}_{n}^{j+n}\right)_{n \geq 0} .
\end{array}
$$

All the coefficients $a_{0}^{j}$ vanish, except for $a_{0}^{0}$, which is given by (33).

The scheme of the algorithm is the following. Suppose $i \geq 1$, and $u^{j}$ and $v^{j}$ are known for $1 \leq j \leq i-1$. The order of operations at step $i$ is the following:

(1) $u_{S}^{i}$ is determined by $\left(\bar{v}^{i-n}\right)_{1 \leq n \leq i}$,

(2) $\bar{u}^{i}$ is determined by $u_{S}^{i}$,

(3) $v_{S}^{i}$ is determined by $\left(\bar{u}^{i-n}\right)_{0 \leq n \leq i}$,

(4) $\bar{v}^{i}$ is determined by $v_{S}^{i}$.

Details are given below.

Initialization:

(S1) Define $a_{0}^{0}$ by (33), and hence $u_{S}^{0}$ by (22).

(S2) From $u_{S}^{0}$, define $\bar{u}^{0}$ by (29), and hence $u^{0}=u_{S}^{0}+\bar{u}^{0}$ is determined.

(S3) Define $\mathrm{b}_{n}^{n}$ for $n \geq 0$ from (20) as the coefficients of $\bar{u}^{0}$; see the next subsection for the practical method. Hence, $v_{S}^{0}=\mathrm{a}_{0}^{0}+\mathrm{b}_{0}^{0} \ln (\rho)$ is determined from (28).

(S4) From $v_{S}^{0}, \bar{v}^{0}$ is computed by (31), and hence $v^{0}=v_{S}^{0}+\bar{v}^{0}$ is determined.

(S5) Define $\mathrm{a}_{n}^{n}$ for $n \geq 1$ from (26) as the coefficients of $\bar{v}^{0}$; see the next subsection for the practical method.

For $i \geq 1$, suppose that $u^{j}$ and $v^{j}$ have been determined, together with the coefficients in (35), for $0 \leq j \leq i-1$.

(R1) Since $\mathrm{a}_{0}^{i}=0$, and writing, for $1 \leq n \leq i, \mathrm{a}_{n}^{i}=\mathrm{a}_{n}^{(i-n)+n}, u_{S}^{i}$ is given by (25), where the coefficients are determined by those of the $\bar{v}^{j}$ for $1 \leq j \leq i-1$.

(R2) $\bar{u}^{i}$ is obtained by solving (30).

(R3) The coefficients $\mathrm{b}_{n}^{i+n}$ for $n \geq 0$ are extracted from $\bar{u}^{i}$ in (23) and (24); see the next subsection for the practical method.

(R4) Since $\mathrm{a}_{0}^{i}=0$, and using $\mathrm{b}_{n}^{i}=\mathrm{b}_{n}^{j+n}$ with $j=i-n, v_{S}^{i}$ is determined from (28).

(R5) $\bar{v}^{i}$ is obtained by solving (32).

(R6) $u^{i}$ and $v^{i}$ are obtained by summing the singular and regular parts.

This iterative method is summarized in Table 2. 


\begin{tabular}{|c|ccccc|}
\hline $\mathrm{a}_{n}^{i} / \mathrm{b}_{n}^{i}$ & $i=0$ & $i=1$ & $i=2$ & $i=3$ & $i=4$ \\
\hline$n=0$ & $(33)$ /Outer 0 & 0/Outer 1 & 0/Outer 2 & 0/Outer 3 & 0/Outer 4 \\
$n=1$ & 0 & Inner 0/Outer 0 & Inner 1/Outer 1 & Inner 2/Outer 2 & Inner 3/Outer 3 \\
$n=2$ & 0 & 0 & Inner 0/Outer 0 & Inner 1/ Outer 1 & Inner 2/Outer 2 \\
$n=3$ & 0 & 0 & 0 & Inner 0/Outer 0 & Inner 1/Outer 1 \\
$n=4$ & 0 & 0 & 0 & 0 & Inner 0/Outer 0 \\
\hline
\end{tabular}

Table 2. Summary of the inductive method to obtain the coefficients $\mathrm{a}_{n}^{i}$ and $\mathrm{b}_{n}^{i}$ : in the corresponding cell is indicated the problem which must be solved.

2.2.7. The practical method for determining the coefficients $\mathrm{a}_{n}^{i}$ and $\mathrm{b}_{n}^{i}$ for $0 \leq n \leq i$. Throughout this section, $\mathscr{b}_{r}$ denotes the arc of the circle of radius $r$ starting on $\Gamma^{-}$ and ending on $\Gamma^{+}$:

$$
\mathscr{C}_{r}=\{(r, \theta): 0 \leq \theta \leq \omega\} .
$$

The coefficients $\mathrm{a}_{n}^{i}$ and $\mathrm{b}_{n}^{i}$ can be obtained by path integrals (which are path independent) as asserted in the following proposition.

Proposition 4. Let $i \geq 0$. Assume that $\bar{v}^{i}$ and $\bar{u}^{i}$ are known. Then:

(1) For $n \geq 1, a_{n}^{i+n}$ is given by the following path integral over $\mathfrak{b}_{\rho}$, which is independent of $\rho$ provided that $\rho>1$ :

$$
a_{n}^{i+n}=\frac{2 \rho^{n \lambda}}{\omega} \int_{0}^{\omega} \bar{v}^{i}(\rho, \theta) \cos (n \lambda \theta) d \theta .
$$

(2) For $n \geq 0, b_{n}^{i+n}$ is given by the following path integral over $\mathscr{C}_{r}$, which is independent of $r$ provided that $0<r<r^{*}$ :

$$
\mathrm{b}_{0}^{i}=\frac{1}{\omega} \int_{0}^{\omega} \bar{u}^{i}(r, \theta) d \theta, \quad \mathrm{b}_{n}^{i+n}=\frac{2 r^{-n \lambda}}{\omega} \int_{0}^{\omega} \bar{u}^{i}(r, \theta) \cos (n \lambda \theta) d \theta \quad \text { for } n \geq 1 .
$$

Proof. The proofs are identical for the two families of coefficients and only that concerning $\mathrm{b}_{n}^{i+n}$ will be given. By (23), $\bar{u}^{i}$ is given for $0<r<r^{*}$ by

$$
\bar{u}^{i}(r, \theta)=\sum_{p \in \mathbb{N}} \mathrm{b}_{p}^{i+p} r^{p \lambda} \cos (p \lambda \theta),
$$

which is for fixed $r$ the Fourier series of $\bar{u}^{i}(r, \cdot)$. Formulas (37) follow.

2.3. Verification in the case of a small cavity. This subsection is devoted to the verification of the construction of the matched asymptotic expansion (MAE) presented in the previous subsections on an example where the exact solution is obtained in a closed form and hence can be directly expanded. Specifically, we consider a Laplace problem posed in a domain which consists of an angular sector 


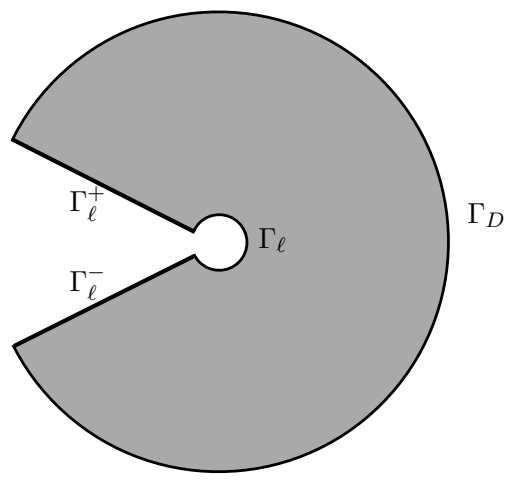

Figure 3. The domain $\Omega_{l}$ in the case of a cavity.

delimited by two arc of circles. The radius of the outer circle is equal to 1 while the radius of the inner circle is $l$; see Figure 3. Thus,

$$
\Omega_{l}=\left\{\boldsymbol{x}=r \cos \theta \boldsymbol{e}_{1}+r \sin \theta \boldsymbol{e}_{2}: r \in(l, 1), \theta \in(0, \omega)\right\} .
$$

The sides of the notch and the inner circle are free and hence the boundary conditions on those parts of the boundary are

$$
\frac{\partial u_{l}}{\partial v}=0 \quad \text { on } \Gamma_{l}^{+} \cup \Gamma_{l}^{-} \cup \Gamma_{l},
$$

where $\Gamma_{l}^{ \pm}=\{(r, \theta): l<r<1, \theta=0$ or $\omega\}, \Gamma_{l}=\{(r, \theta): r=l, 0 \leq \theta \leq \omega\}$. (Note that $\Gamma_{l}^{ \pm}$depend on $l$, contrary to the assumption made in the remaining part of the paper. But that has no influence on the results.) The displacement is prescribed on the outer boundary $\Gamma_{D}$ so that

$$
u_{l}(\boldsymbol{x})=\cos \lambda \theta \quad \text { on } \Gamma_{D}, \quad \lambda=\frac{\pi}{\omega} .
$$

Note that $\Gamma_{N}$ is empty. Assuming that there is no body force, the exact solution of this antiplane elastic problem is given by

$$
u_{l}(\boldsymbol{x})=\left(\frac{l^{2 \lambda}}{1+l^{2 \lambda}} r^{-\lambda}+\frac{1}{1+l^{2 \lambda}} r^{\lambda}\right) \cos \lambda \theta
$$

Inserting the Taylor series of $1 /\left(1+l^{2 \lambda}\right)=\sum_{i \in \mathbb{N}}(-1)^{i}\left(l^{2 \lambda}\right)^{i}$ for $l<1$, the expansion of $u_{l}$ at a given $\boldsymbol{x}$ takes the form

$$
u_{l}(\boldsymbol{x})=r^{\lambda} \cos \lambda \theta+\sum_{n \in \mathbb{N}^{*}} l^{2 n \lambda}\left(r^{-\lambda}-r^{\lambda}\right) \cos \lambda \theta .
$$


Thus (41) corresponds to the outer expansion where the odd terms vanish and the even terms are given by

$$
u^{0}(\boldsymbol{x})=r^{\lambda} \cos \lambda \theta, \quad u^{2 n}(\boldsymbol{x})=(-1)^{n}\left(r^{\lambda}-r^{-\lambda}\right) \cos \lambda \theta \quad \text { for all } n \geq 1 .
$$

To obtain the inner expansion, replace $r$ by $l \rho$ in (40), to get

$$
u_{l}(l \boldsymbol{y})=\frac{l^{\lambda}}{1+l^{2 \lambda}}\left(\rho^{-\lambda}+\rho^{\lambda}\right) \cos \lambda \theta .
$$

Inserting the Taylor series as before, the expansion of $u_{l}(l \boldsymbol{y})$ is given by

$$
u_{l}(l \boldsymbol{y})=\sum_{n \in \mathbb{N}}(-1)^{n} l^{(2 n+1) \lambda}\left(\rho^{-\lambda}+\rho^{\lambda}\right) \cos \lambda \theta,
$$

which corresponds to the inner expansion where the even terms vanish and the odd terms are given by

$$
v^{2 n+1}(\boldsymbol{y})=(-1)^{n}\left(\rho^{-\lambda}+\rho^{\lambda}\right) \cos \lambda \theta \text { for all } n \geq 0 .
$$

It remains to be checked that the procedure described in the previous subsections yields the same coefficients. Since $g=0, a_{0}^{i}=0$ for all $i \geq 0$ and there is no logarithmic singularity; see Remark 4. The details for the first steps of the procedure are given below.

(S1) By (33), $a_{0}^{0}=0$ and hence $u_{S}^{0}=0$.

(S2) Hence (29) becomes: $\Delta u^{0}=0$ in $\Omega_{0}, \partial u^{0} / \partial \theta=0$ on $\theta \in\{0, \omega\}, u^{0}=\cos \lambda \theta$ on $r=1$. The unique solution in $H^{1}\left(\Omega_{0}\right)$ is $u^{0}$ given by (42).

(S3) By (37), $\mathrm{b}_{1}^{1}=1$ and $\mathrm{b}_{n}^{n}=0$ for $n \neq 1$. Hence $v_{S}^{0}=0$.

(S4) Since $v_{S}^{0}=0$ and $g=0$, (31) gives $\bar{v}^{0}=0$ and hence $v^{0}=0$.

(S5) By (36), $\mathrm{a}_{n}^{n}=0$ for $n \geq 1$.

(S6) By (25), $u_{S}^{1}=0$.

(S7) By (30), $\bar{u}^{1}=0$ and hence $u^{1}=0$.

(S8) By (37), $\mathrm{b}_{n}^{n+1}=0$ for all $n$. Hence $v_{S}^{1}=\rho^{\lambda} \cos \lambda \theta$.

(S9) Hence (32) for $i=1$ becomes: $\Delta \bar{v}^{1}=0$ in $\Omega^{\infty}, \partial \bar{v}^{1} / \partial \theta=0$ on $\theta \in\{0, \omega\}$, $\partial \bar{v}^{1} / \partial \rho=-\lambda \cos \lambda \theta$ on $\rho=1$. The unique regular solution is $\bar{v}^{1}=\rho^{\lambda} \cos \lambda \theta$ and hence $v^{1}$ is given by (45).

(S10) By (36), $\mathrm{a}_{1}^{2}=1$ and $\mathrm{a}_{n}^{n+1}=0$ for $n \neq 1$.

(S11) By (25), $u_{S}^{2}=r^{-\lambda} \cos \lambda \theta$.

(S12) Hence (30) for $i=2$ becomes: $\Delta \bar{u}^{2}=0$ in $\Omega_{0}, \partial \bar{u}^{2} / \partial \theta=0$ on $\theta \in\{0, \omega\}$, $\bar{u}^{2}=-\cos \lambda \theta$ on $r=1$. The unique solution in $H^{1}\left(\Omega_{0}\right)$ is $\bar{u}^{2}=-r^{\lambda} \cos \lambda \theta$ and hence $u^{2}$ is given by (42). 


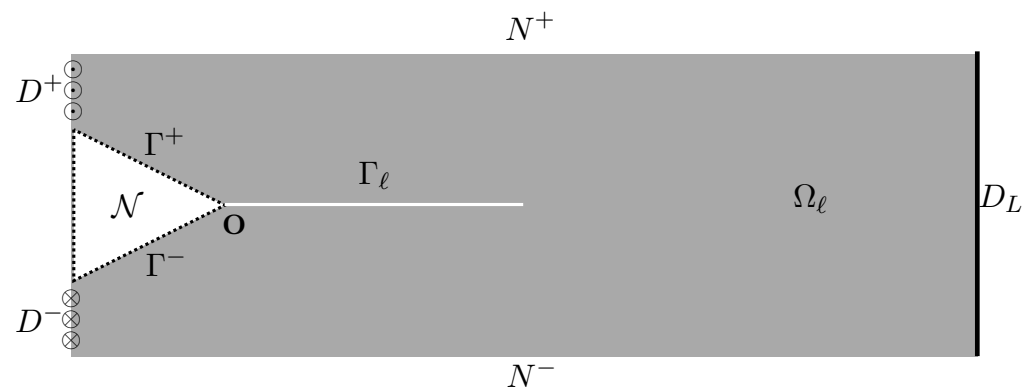

Figure 4. Definition of the cracked notch-shaped body $\Omega_{l}$ with the various parts of the boundary.

Proceeding by induction, the expected expansions are finally recovered. The end of the verification is left to the reader.

\section{Application to the case of a crack}

3.1. Setting the problem. In this section, the method is applied to a defect which is a noncohesive crack. Specifically, let $\Omega$ be the rectangle $(-H, L) \times(-H,+H)$. Let $\epsilon$ be a given parameter in $\left.(0,1), \mathcal{N}=\left\{\boldsymbol{x}=\left(x_{1}, x_{2}\right):-H<x_{1} \leq 0,\left|x_{2}\right| \leq \epsilon\left|x_{1}\right|\right)\right\}$. The notch-shaped body is $\Omega_{0}=\Omega \backslash \mathcal{N}$. Finally the cracked body $\Omega_{l}$ is obtained by removing from $\Omega_{0}$ the line segment $\Gamma_{l}=(0, l) \times\{0\}$; see Figure 4 .

The boundary $\Gamma_{D}$ where the displacement is prescribed corresponds to the sides $D^{ \pm}$and $D_{L}$, with boundary conditions

$$
u_{l}(\boldsymbol{x})=\left\{\begin{array}{cl}
+H & \text { on } D^{+}=\{-H\} \times[\epsilon H, H], \\
-H & \text { on } D^{-}=\{-H\} \times[-H,-\epsilon H], \\
0 & \text { on } D_{L}=\{L\} \times[-H, H] .
\end{array}\right.
$$

The remaining parts of the boundary (including the lips of the crack) are free; that is,

and

$$
\frac{\partial u_{l}}{\partial x_{2}}= \begin{cases}0 & \text { on } \Gamma_{l}=(0, l) \times\{0\} \\ 0 & \text { on } N^{ \pm}=(-H, L) \times\{ \pm H\}\end{cases}
$$

$$
\frac{\partial u_{l}}{\partial n}=0 \quad \text { on } \Gamma^{ \pm}=\left\{\left(x_{1}, x_{2}\right):-H<x_{1}<0, x_{2}= \pm \epsilon x_{1}\right\} .
$$

Remark 5. The amplitude of the prescribed displacement is normalized to $H$ so that $u_{l}$ has the dimension of a length. The fact that the amplitude is equal to the height $H$ has no importance in the present context of linearized elasticity. We will introduce a time-dependent amplitude of the prescribed displacement when we study the propagation of the crack. Then the prescribed displacement will take "reasonable" values, controlled by the toughness of the material. 
Remark 6. The case $\epsilon=0$ corresponds to a body with an initial crack of length $H$ and this limiting case is also considered in this paper. The case $\epsilon=1$ corresponds to a corner with an angle $\pi / 2$, the sides $D^{ \pm}$being reduced to the points $(-H, \pm H)$. This limiting case will not be considered here.

Remark 7. We only consider the case where the crack path is the line segment $(0, L) \times\{0\}$. It is a rather natural assumption by virtue of the symmetry of the geometry and the loading. An interesting extension should be to consider nonsymmetric geometry or loading and hence to take the direction of the crack as a parameter. This extension is reserved for future works.

We are in the case where $g=0$ on $\Gamma_{l}$. Therefore, by virtue of Proposition 3, all the coefficients $a_{0}^{i}$ vanish and there are no logarithmic singularities. Accordingly, the solution can be expanded as follows:

Outer expansion: $\quad u_{l}(\boldsymbol{x})=u^{0}(\boldsymbol{x})+l^{\lambda} u^{1}(\boldsymbol{x})+l^{2 \lambda} u^{2}(\boldsymbol{x})+l^{3 \lambda} u^{3}(\boldsymbol{x})+\cdots$,

Inner expansion: $\quad u_{l}(\boldsymbol{x})=v^{0}(\boldsymbol{y})+l^{\lambda} v^{1}(\boldsymbol{y})+l^{2 \lambda} v^{2}(\boldsymbol{y})+l^{3 \lambda} v^{3}(\boldsymbol{y})+\cdots$, with

$$
\lambda=\frac{\pi}{\omega} \quad \text { and } \quad \omega=2 \pi-2 \arctan (\epsilon) .
$$

By symmetry of the geometry and the loading, the real field $u_{l}$ is an odd function of $x_{2}$; i.e.,

$$
u_{l}\left(x_{1},-x_{2}\right)=-u_{l}\left(x_{1}, x_{2}\right), \quad u_{l}(r, \omega-\theta)=-u_{l}(r, \theta) .
$$

Therefore, all the fields $u^{i}, \bar{u}^{i}, v^{i}, \bar{v}^{i}$ admit the same symmetry. Therefore, by Proposition 4, all coefficients $b_{2 n}^{i+2 n}$ and $a_{2 n}^{i+2 n}$ vanish. Consequently, the odd terms of the outer expansion and the even terms of the inner expansions vanish; i.e., $u^{2 i+1}=0$ and $v^{2 i}=0$ for all $i \in \mathbb{N}$. Finally, the solution admits the following expansions:

Outer expansion: $u_{l}(\boldsymbol{x})=\sum_{i \in \mathbb{N}} l^{2 i \lambda} u^{2 i}(\boldsymbol{x})$,

Inner expansion: $\quad u_{l}(\boldsymbol{x})=\sum_{i \in \mathbb{N}} l^{(2 i+1) \lambda} v^{2 i+1}(\boldsymbol{y})$.

By symmetry, the following coefficients vanish:

$\mathrm{a}_{n}^{i}=0 \quad$ when $n$ or $i-n$ are even, $\quad \mathrm{b}_{n}^{i}=0 \quad$ when $n$ is even or $i-n$ is odd.

Examine now the singularities of $\nabla u_{l}$ (in the sense that $\nabla u_{l}$ is not bounded) according to whether or not $l=0$, and according to whether or not $\epsilon=0$. 
(1) When $\epsilon>0$ and $l=0$. Then $\nabla u_{0}$ is infinite at the tip of the notch and in its neighborhood has the form

$$
\nabla u_{0}(\boldsymbol{x})=\frac{\lambda \mathrm{b}_{1}^{1}}{r^{1-\lambda}}\left(\cos (\lambda \theta) \boldsymbol{e}_{r}-\sin (\lambda \theta) \boldsymbol{e}_{\theta}\right)+\text { regular terms }
$$

(2) When $\epsilon>0$ and $l>0$. Then $\nabla u_{l}$ is no longer infinite at the tip of the notch but becomes infinite at the tip of the crack, with the usual singularity in $1 / \sqrt{r}$; see [Bui 1978]. Specifically, $\nabla u_{l}$ has the form

$$
\nabla u_{l}(\boldsymbol{x})=\frac{K_{l}}{\mu \sqrt{2 \pi r^{\prime}}}\left(\sin \left(\frac{\theta^{\prime}}{2}\right) \boldsymbol{e}_{r}+\cos \left(\frac{\theta^{\prime}}{2}\right) \boldsymbol{e}_{\theta}\right)+\text { regular terms } .
$$

In (50), $\left(r^{\prime}, \theta^{\prime}\right)$ denotes the polar coordinate system with $\boldsymbol{x}=\left(l+r^{\prime} \cos \theta^{\prime}\right) \boldsymbol{e}_{1}+$ $r \sin \theta^{\prime} \boldsymbol{e}_{2}$ and the angular function of $\theta^{\prime}$ is normalized so that $K_{l}$ be the usual stress intensity factor. $K_{l}$ depends on $l$ and is "strongly" influenced by the presence of the notch when $l$ is small. (In fact, $K_{l}$ goes to 0 when $l$ goes to 0 as we will see below.) So, even if the stresses are only singular at the tip of the crack, there is a kind of overlapping of the previous singularity at the tip of the notch. This phenomenon renders the computations by the finite element method less accurate when $l$ is small.

(3) When $\epsilon=0$. Then the notch is already a crack and it is unnecessary to treat separately $l=0$ and $l>0$. In any case $\nabla u_{l}$ has the classical singularity in $1 \sqrt{r}$ as in (50) and there is no more overlapping of two singularities. The computations by the finite element method are accurate in the full range of values of $l$.

3.2. The issue of the computation of the energy release rate. The main goal of this section is to obtain accurate values for the elastic energy $\mathscr{P}_{l}$ stored in the cracked body and for its derivative with respect to $l$, the so-called energy release rate $\varphi_{l}$, when $l$ is small. By definition, the elastic energy is given by

$$
\mathscr{P}_{l}=\frac{1}{2} \int_{\Omega_{l}} \mu \nabla u_{l} \cdot \nabla u_{l} d x .
$$

By virtue of Clapeyron's formula, the elastic energy stored in the body when the body is at equilibrium is equal to one half the work done by the external loads over the prescribed displacement on $D^{ \pm}$. Therefore, using the symmetry of $u_{l}$, the elastic energy can also be written as an integral over $D^{+}$:

$$
\mathscr{P}_{l}=-\int_{\epsilon H}^{H} \mu H \frac{\partial u_{l}}{\partial x_{1}}\left(-H, x_{2}\right) d x_{2},
$$

which involves only the displacement field far from the tip of the notch.

By definition (see [Bourdin et al. 2008; Leblond 2003]), the energy release rate $\mathscr{G}_{l}$ is the opposite of the derivative of the elastic energy with respect to the 


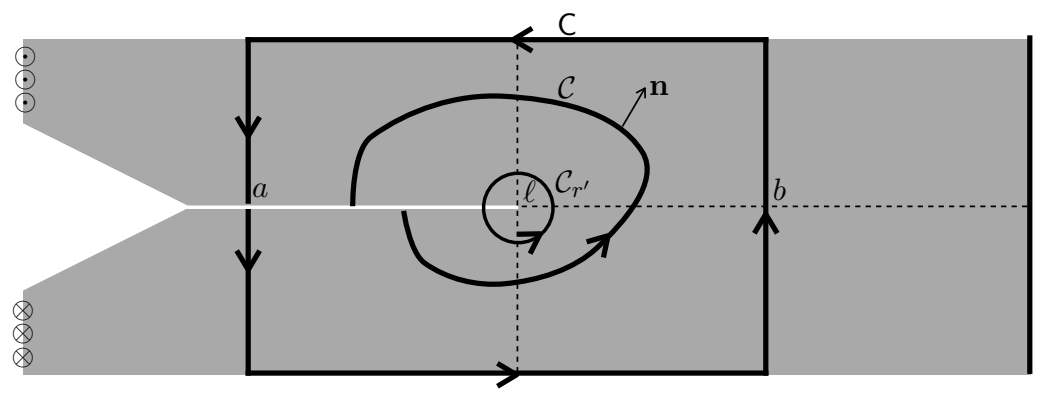

Figure 5. Examples of paths for which $\mathscr{g}_{\mathscr{C}}$ is equal to $\mathscr{G}_{l}$.

length of the crack:

$$
\varphi_{l}=-\frac{d \mathscr{P}_{l}}{d l} .
$$

Even though $\mathscr{P}_{l}$ involves the $l$-dependent displacement field $u_{l}$, its derivative does not involve the derivative $d u_{l} / d l$ but can be expressed in terms of $u_{l}$ only. This property is a consequence of the fact that $u_{l}$ satisfies the equilibrium equations. Specifically, $\mathscr{G}_{l}$ can be computed either with the help of path integrals like the $\mathscr{g}$ integral of [Rice 1968] or by using the so-called $G-\theta$ method developed in [Destuynder and Djaoua 1981]. We recall below the main ingredients of both methods when $0<l<L$. The cases $l=0$ and $l=L$ are treated separately.

In the former method, the integral $\mathscr{E}_{\mathscr{C}}$ over the path $\mathscr{C}$ is defined by

$$
\mathscr{\Phi}_{\mathscr{C}}=\int_{\mathscr{C}}\left(\frac{\mu}{2} \nabla u_{l} \cdot \nabla u_{l} n_{1}-\mu \frac{\partial u_{l}}{\partial n} \frac{\partial u_{l}}{\partial x_{1}}\right) d s,
$$

where $\boldsymbol{n}$ denotes the outer normal of the path. This integral is (theoretically) pathindependent and equal to $\mathscr{G}_{l}$ provided that the path $\mathscr{b}$ starts from the lip of the crack, circumvents the tip of the crack and finishes on the lip of the crack like in Figure 5; see [Bui 1978]. This path independence is used to obtain Irwin's formula [Irwin 1958; Leblond 2003]. Indeed, taking for path the circle $\mathscr{C}_{r^{\prime}}$ centered at the tip of the crack with radius $r^{\prime}$, using (50) and passing to the limit when $r^{\prime} \rightarrow 0$, the following link between the energy release rate and the stress intensity factor $K_{l}$ introduced in (50) is obtained:

$$
\mathscr{G}_{l}=\lim _{r^{\prime} \rightarrow 0} \mathscr{\mathscr { C }}_{\mathscr{C}_{r^{\prime}}}=\frac{K_{l}^{2}}{2 \mu} .
$$

For the computations, the particularities of the geometry and of the loading can be exploited, to choose a path made of line segments parallel to the axes like the path $\mathrm{C}$ in Figure 5:

$\mathrm{C}=\{a\} \times(-H, 0) \cup[a, b] \times\{-H\} \cup\{b\} \times(-H, H) \cup[a, b] \times\{+H\} \cup\{a\} \times(0, H)$ 
with $0<a<l<b<L$. Then $\mathscr{g}_{C}=\varphi_{l}$. Therefore, since $n_{1}=0$ and $\partial u_{l} / \partial n=0$ on the sides $x_{2}= \pm H$ and by virtue of the symmetry of $u_{l}, \mathscr{G}_{l}$ takes the form

$$
\begin{aligned}
\mathscr{G}_{l}=\mu \int_{\{b\} \times(0, H)}\left(\left(\frac{\partial u_{l}}{\partial x_{2}}\right)^{2}-\left(\frac{\partial u_{l}}{\partial x_{1}}\right)^{2}\right) d x_{2} & \\
& \quad-\mu \int_{\{a\} \times(0, H)}\left(\left(\frac{\partial u_{l}}{\partial x_{2}}\right)^{2}-\left(\frac{\partial u_{l}}{\partial x_{1}}\right)^{2}\right) d x_{2} .
\end{aligned}
$$

From a theoretical point of view, $a$ and $b$ can be chosen arbitrarily, provided that they satisfy the constraints above. Indeed, the integral over the line segment $x_{1}=a$ (respectively, $x_{1}=b$ ) does not depend on $a$ (respectively, on $b$ ) because $u_{l}$ is harmonic and satisfies homogeneous Neumann boundary conditions on $N^{ \pm}$and $\Gamma_{l}$. (This verification is left to the reader; see [Marigo 2010, Proposition 8] for a proof.) However, from a numerical point of view, this is no longer true because the computed displacement field does not satisfy exactly the equilibrium equations. Consequently, the computed values of $\varphi_{l}$ depend on the choice of $a$ and $b$. Moreover, since the integral over the line $a$ involves the gradient of the displacement, this integral can be badly approximated when $l$ is small because of the singularity.

The $G-\theta$ method is based on a change of variables which sends the $l$-dependent domain $\Omega_{l}$ onto a fixed domain. In essence, it is the basic method to prove that $l \mapsto \mathscr{P}_{l}$ is differentiable; see [Destuynder and Djaoua 1981] for the genesis of this method and [Chambolle et al. 2010] for a discussion on a generalization of the concept of energy release rate. In turn the $G-\theta$ approach gives a practical method to compute the energy release rate; see the previous two references. Specifically, for a given $l>0$, we associate to a Lipschitz continuous vector field $\boldsymbol{\theta}$ defined on $\Omega_{l}$ the volume integral

$$
\mathrm{G}_{\boldsymbol{\theta}}=\int_{\Omega_{l}}\left(\sum_{i, j=1}^{2} \mu \frac{\partial \theta_{i}}{\partial x_{j}} \frac{\partial u_{l}}{\partial x_{i}} \frac{\partial u_{l}}{\partial x_{j}}-\frac{\mu}{2} \nabla u_{l} \cdot \nabla u_{l} \operatorname{div} \boldsymbol{\theta}\right) d x
$$

It can shown that, if $\boldsymbol{\theta}$ is such that $\boldsymbol{\theta}(l, 0)=\boldsymbol{e}_{1}$ and $\boldsymbol{\theta} \cdot \boldsymbol{n}=0$ on $\partial \Omega_{l}$, then $\mathrm{G}_{\boldsymbol{\theta}}$ is independent of $\boldsymbol{\theta}$ and equal to $\mathscr{G}_{l}$. Of course, this result of independence holds only when $u_{l}$ is the true displacement field. If it is numerically approximated, then $\mathrm{G}_{\theta}$ becomes $\boldsymbol{\theta}$ dependent. In our case, owing to the simplicity of the geometry, we can use a very simple vector field $\boldsymbol{\theta}$ which renders the computations easier. Specifically, let $\boldsymbol{\theta}$ be given by

$$
\boldsymbol{\theta}(\boldsymbol{x})=\left\{\begin{array}{cl}
\boldsymbol{\Gamma} & \text { if } x_{1}<0, \\
\frac{x_{1}}{l} \boldsymbol{e}_{1} & \text { if } 0 \leq x_{1} \leq l, \\
\frac{L-x_{1}}{L-l} \boldsymbol{e}_{1} & \text { if } l \leq x_{1}<L .
\end{array}\right.
$$


It satisfies the required conditions and hence $\mathrm{G}_{\theta}=\mathscr{G}_{l}$. Accordingly, owing to the symmetry, $\mathscr{G}_{l}$ takes the form

$$
\begin{aligned}
\mathscr{\varphi}_{l}=\frac{\mu}{L-l} \int_{l}^{L} \int_{0}^{H}\left(\left(\frac{\partial u_{l}}{\partial x_{2}}\right)^{2}\right. & \left.-\left(\frac{\partial u_{l}}{\partial x_{1}}\right)^{2}\right) d x_{2} d x_{1} \\
& -\frac{\mu}{l} \int_{0}^{l} \int_{0}^{H}\left(\left(\frac{\partial u_{l}}{\partial x_{2}}\right)^{2}-\left(\frac{\partial u_{l}}{\partial x_{1}}\right)^{2}\right) d x_{2} d x_{1} .
\end{aligned}
$$

Comparing (56) with (54), (56) can be seen as an average of all the line integrals appearing in (54) when $a$ and $b$ vary, respectively, from 0 to $l$ and from $l$ to $L$. Accordingly, it can be expected that (56) gives more accurate computations than (54) when $l$ is small.

3.3. Numerical results obtained for $\mathscr{G}_{l}$ by the FEM. All the computations based on the finite element method are implemented in the industrial code COMSOL. They are performed after introducing dimensionless quantities. Specifically, in all the computations, the dimensions of the body are $H=1$ and $L=5$, the shear modulus $\mu=1$. That does not restrict the generality of the study because the scale dependencies are known in advance. Indeed, the true physical quantities are related to the normalized quantities (denoted with a tilde) by

$$
l=H \tilde{l}, \quad u_{l}=H \tilde{u}_{l}, \quad \mathscr{P}_{l}=\mu H^{2} \tilde{\mathscr{P}}_{l}, \quad \varphi_{l}=\mu H \tilde{\mathscr{G}}_{l} .
$$

For a given $\tilde{l} \in(0,5)$ and a given $\epsilon \in(0,1)$, we use the symmetry of the body and of the load to mesh only its upper half and prescribe $\tilde{u}_{l}=0$ on the segment $\tilde{l} \leq \tilde{x}_{1} \leq 5$, $\tilde{x}_{2}=0$. We use 6-node triangular elements, i.e., quadratic Lagrange interpolations. The mesh is refined near the singular corners and a typical mesh contains 25000 elements and 50000 degrees of freedom. We compute the discretized solution (still denoted) $\tilde{u}_{l}$ by solving the linear system. Then, the energy $\tilde{\mathscr{P}}_{l}$ and the energy release rate $\tilde{\mathscr{G}}_{l}$ are obtained by postprocessing. The energy is obtained by a direct integration of the elastic energy density over the body. The derivative of the energy is obtained by using formula (56), which needs to integrate the different parts of the elastic energy density over the two rectangles $(0, \tilde{l}) \times(0,1)$ and $(\tilde{l}, 5) \times(0,1)$. For a given $\epsilon$, we compute $\tilde{\mathscr{P}}_{l}$ and $\tilde{\mathscr{G}}_{l}$ for $\tilde{l}$ varying from 0.001 to 5 , first by steps of 0.001 in the interval $(0,0.05)$, then by steps of 0.002 in the interval $(0.05,0.2)$, finally by steps of 0.01 in the interval $(0.2,5)$. The computations can be considered sufficiently accurate for $\tilde{l} \geq 0.002$, even if this lower bound depends on $\epsilon$, the computations being less accurate for small (but nonzero) values of $\epsilon$. Below this value, if we try to refine the mesh near the corner of the notch, the results become mesh-sensitive, and the linear system becomes ill-conditioned. Since only the part of the graph of $\tilde{\mathscr{G}}_{l}$ close to $\tilde{l}=0$ is interesting when $\epsilon$ is small, we cannot obtain 


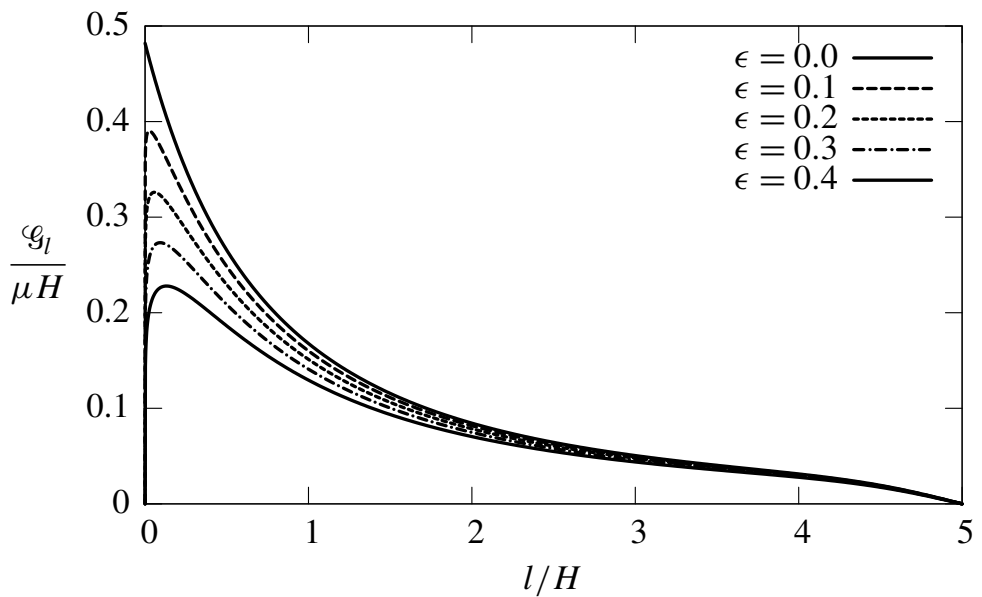

Figure 6. Computation by the Finite Element Method of the energy release rate $\varphi_{l}$ as a function of the crack length $l$ for five values of the notch angle.

accurate results when $\epsilon$ is too small. (Of course, this remark does not apply when $\epsilon=0$, because $\tilde{l}=0$ is not a "singular" case.)

The cases $\tilde{l}=0$ and $\tilde{l}=5$ with $\epsilon \neq 0$ are treated with specific meshes. We have only to compute $\tilde{u}_{0}, \tilde{\mathscr{P}}_{0}, \tilde{u}_{L}$ and $\tilde{\mathscr{P}}_{L}$, since $\tilde{\varphi}_{0}=\tilde{\mathscr{G}}_{L}=0$.

The case $\epsilon=0$ is treated separately by adapting the previous methods. In particular, to calculate $\tilde{\mathscr{G}}_{l}$, the second integral in (56) is replaced by an integral over the rectangle $(-1,0) \times(0,1)$, and this integral is divided by $1+\tilde{l}$ instead of $\tilde{l}$. Moreover, the mesh is refined only near the tip of the crack; $\tilde{l}=0$ is no longer a particular case and the computations of $\tilde{\varphi}_{l}$ are accurate in the full range of $\tilde{l}$.

Let us highlight the main features of the numerical results plotted in Figure 6. These properties will be the basic assumptions from which we study the crack propagation at the end of the present section.

(P1) For $\epsilon=0, \mathscr{G}_{l} / \mu H$ is monotonically decreasing from 0.4820 to 0 when $l / H$ grows from 0 to 5 .

(P2) For $\epsilon>0, \mathscr{G}_{l} / \mu H$ starts from 0 at $l / H=0$, then is rapidly increasing. This growth is of such magnitude (for instance, $\mathscr{G}_{l} / \mu H=0.1443$ when $l / H=$ 0.002 for $\epsilon=0.4$ ) that it cannot be correctly captured by the FEM.

(P3) Still for $\epsilon>0, \mathscr{G}_{l}$ is monotonically increasing as long as $l \leq l_{\mathrm{m}}$. At $l=l_{\mathrm{m}}, \mathscr{G}$ takes its maximal value $G_{m}$. Those values which depend on $\epsilon$ are given in the table below. It turns out that $l_{\mathrm{m}} / H$ is rather small. 


\begin{tabular}{|c|ccccc|}
\hline$\epsilon$ & 0 & 0.1 & \multicolumn{1}{c}{0.2} & \multicolumn{1}{c}{0.3} & 0.4 \\
\hline$l_{\mathrm{m}} / H$ & 0 & 0.024 & 0.058 & 0.092 & 0.130 \\
$\mathrm{G}_{\mathrm{m}} / \mu H$ & 0.4820 & 0.3900 & 0.3260 & 0.2733 & 0.2279 \\
\hline
\end{tabular}

(P4) For $\epsilon>0$ again, $\mathscr{G}_{l}$ is monotonically decreasing from $\mathrm{G}_{\mathrm{m}}$ to 0 when $l$ grows from $l_{\mathrm{m}}$ to $5 H$.

3.4. Evaluation of the energy release rate by the MAM. By virtue of (52), $\mathscr{P}_{l}$ can be expanded by using the outer expansion of $u_{l}$. Using (47) leads to

$$
\mathscr{P}_{l}=\sum_{i \in \mathbb{N}} P_{2 i}\left(\frac{l}{H}\right)^{2 i \lambda} \mu H^{2},
$$

where the coefficients $P_{2 i}$ of the expansions are dimensionless. The expansion of the energy release rate can be immediately deduced from that of the energy:

$$
\mathscr{G}_{l}=-\sum_{i \in \mathbb{N}^{*}} 2 i \lambda P_{2 i}\left(\frac{l}{H}\right)^{2 i \lambda-1} \mu H,
$$

and it is not necessary to use the path integrals $\mathscr{F}_{\mathscr{C}}$ or the $G-\theta$ method. Let us remark that

$$
\mathscr{G}_{0}=\left\{\begin{array}{cc}
0 & \text { if } \epsilon \neq 0, \\
-P_{2} \mu H=K_{0}^{2} / 2>0 & \text { if } \epsilon=0,
\end{array}\right.
$$

because $\lambda>1 / 2$ in the former case while $\lambda=1 / 2$ in the latter.

To obtain the $i$-th term of the expansion of $\mathscr{P}_{l}$ and $\mathscr{G}_{l}$, both the singular part $u_{S}^{i}$ and the regular part $\bar{u}^{i}$ of $u^{i}$ must be recovered. The singular part involves the coefficients $\mathrm{a}_{n}^{i}$ for $1 \leq n \leq i$ which are obtained as the regular parts of the $v^{j}$ for $j \leq i$; see Section 2.2.6. Therefore, the inner problems must be solved to determine the coefficients $\mathrm{b}_{n}^{i}$ for $0 \leq n \leq i$. In practice, these coefficients are obtained by using Proposition 4 after the inner and the outer problems have been solved with a finite element method. The advantage is that those problems do not contain a small defect and the accuracy is guaranteed. The drawback is that more and more problems have to be solved, in order to obtain accurate values of $\varphi_{l}$ when $l / H$ is not small.

In Tables 3 and 4 are given the computed values of the first coefficients of the inner and outer expansions (still with $H=1, L=5, \mu=1$ ). These tables contain all the terms which are necessary to compute the expansions of the energy up to the sixth order, i.e., $P_{2 i}$ for $i \in\{0,1,2,3\}$. (Note that $P_{0}$ does not appear in the expansion of $\varphi_{l}$.) The graphs of $l \mapsto \mathscr{G}_{l}$ obtained from these expansions are plotted in Figure 7 in the cases $\epsilon=0.2$ and $\epsilon=0.4$. They are compared with the values obtained directly by the finite element code COMSOL. From these comparisons, the following conclusions can be drawn: 


\begin{tabular}{cccccccccc}
$\epsilon$ & $\mathrm{a}_{1}^{2}$ & $P_{2}$ & $\mathrm{a}_{1}^{4}$ & $\mathrm{a}_{3}^{4}$ & $P_{4}$ & $\mathrm{a}_{1}^{6}$ & $\mathrm{a}_{3}^{6}$ & $\mathrm{a}_{5}^{6}$ & $P_{6}$ \\
\hline 0 & -0.3930 & -0.4820 & 0.1888 & 0.0987 & 0.3282 & -0.1365 & -0.0537 & -0.0494 & -0.2013 \\
0.1 & -0.3756 & -0.4413 & 0.1766 & 0.0943 & 0.3001 & -0.1279 & -0.0507 & -0.0472 & -0.1931 \\
0.2 & -0.3559 & -0.3957 & 0.1619 & 0.0893 & 0.2673 & -0.1165 & -0.0470 & -0.0446 & -0.1787 \\
0.3 & -0.3342 & -0.3486 & 0.1453 & 0.0838 & 0.2320 & -0.1029 & -0.0427 & -0.0418 & -0.1603 \\
0.4 & -0.3106 & -0.3005 & 0.1273 & 0.0778 & 0.1952 & -0.0880 & -0.0380 & -0.0389 & -0.1385 \\
\hline
\end{tabular}

Table 3. The computed values of the (nonzero) coefficients $a_{n}^{i}$ for $1 \leq n \leq i \leq 6$ and of the leading terms $P_{2}, P_{4}$ and $P_{6}$ of the expansion of the potential energy for several values of the angle of the notch.

\begin{tabular}{ccccccc}
$\epsilon$ & $\mathrm{b}_{1}^{1}$ & $\mathrm{~b}_{1}^{3}$ & $\mathrm{~b}_{3}^{3}$ & $\mathrm{~b}_{1}^{5}$ & $\mathrm{~b}_{3}^{5}$ & $\mathrm{~b}_{5}^{5}$ \\
\hline 0 & -0.7834 & 0.2384 & -0.2059 & -0.1943 & 0.1058 & -0.0172 \\
0.1 & -0.7482 & 0.2091 & -0.2085 & -0.1730 & 0.0992 & -0.0283 \\
0.2 & -0.7089 & 0.1777 & -0.2081 & -0.1489 & 0.0905 & -0.0379 \\
0.3 & -0.6657 & 0.1451 & -0.2045 & -0.1232 & 0.0800 & -0.0454 \\
0.4 & -0.6187 & 0.1125 & -0.1977 & -0.0974 & 0.0683 & -0.0508 \\
\hline
\end{tabular}

Table 4. The computed values of the (nonzero) coefficients $\mathrm{b}_{n}^{i}$ for $1 \leq n \leq i \leq 5$ for several values of the angle of the notch.

(C1) For very small values of $l$, the first nontrivial term (corresponding to $i=1$ in (59)) of the matched asymptotic expansion (denoted by MAM 2 in Figure 7) is sufficient to well approximate $\varphi_{l}$ while the FEM is unable to deliver accurate values.

(C2) For values of $l$ of the order of $l_{\mathrm{m}}$, at least the first two nontrivial terms (corresponding to $i=1$ and 2 in (59)) of the MAE (denoted by MAM 4 in Figure 7) are necessary to capture the change of monotonicity of $\mathscr{G}_{l}$. Indeed, the first term, being monotonically increasing, is unable, alone, to capture that change of behavior.

(C3) Still for values of $l$ of the order of $l_{\mathrm{m}}$, the first two terms are really sufficient to well approximate $\varphi_{l}$ provided that $l_{\mathrm{m}} / H$ is sufficiently small. Specifically, the first two terms are sufficient as long as $l / H<0.2$.

(C4) Accordingly, the approximation of $\varphi_{l}$ by the first two nontrivial terms of the MAE can be used, in the range $\left[0,2 l_{\mathrm{m}}\right]$ of $l$ when $\epsilon \in(0,0.4)$.

(C5) As $l / H$ grows beyond 0.2, more and more terms of the MAE must be added, in order to get a good approximation of $\mathscr{G}_{l}$. Consequently, in the range of 
"large" values of $l / H$, the direct FEM is more accurate and hence is better to use.

\section{Application to the determination of the nucleation of the crack}

The theoretical and numerical results obtained in the previous sections are used here to study the delicate issue of the nucleation of a crack in a sound body or the most classical question of the onset of a preexisting crack. Specifically, we consider the notched body $\Omega_{0}$ which either contains a preexisting crack $l_{0}>0$ or is sound; i.e., $l_{0}=0$. We have also to distinguish different cases according to whether $\epsilon=0$ or $\epsilon>0$. The nucleation or the onset of cracking is governed by either the so-called G-law or the so-called FM-law and one goal of this section is to compare those laws. The interested reader can also refer to [Bourdin et al. 2008; Francfort and Marigo 1998; Negri 2010; Negri and Ortner 2008; Marigo 2010] where other comparisons between the G-law and the FM-law are proposed.

The notched body is submitted to a time-dependent loading process which consists of a monotonically increasing amplitude of the displacement prescribed on the sides $D^{ \pm}$. Specifically, consider the new boundary conditions

$$
u= \pm t H \quad \text { on } D^{ \pm}, \quad t \geq 0 .
$$

The others remain unchanged. (Note that the "time" parameter $t$ is dimensionless.) The evolution problem consists of finding the time evolution of the length of the crack, i.e., $t \mapsto l(t)$ for $t \geq 0$, under the initial condition $l(0)=l_{0} \in[0, L)$. For that, we first remark that, for a given time $t \geq 0$ and a given crack length $l \in[0, L]$, the displacement field which equilibrates the body is

$$
u(t, l)=t u_{l},
$$

where $u_{l}$ is the displacement field introduced in Section 3.1. Accordingly, the potential energy and the energy release rate at time $t$ with a crack length $l$ can be expressed as

$$
\mathscr{P}(t, l)=t^{2} \mathscr{P}_{l}, \quad \mathscr{G}(t, l)=t^{2} \mathscr{G}_{l}
$$

where $\mathscr{P}_{l}$ and $\varphi_{l}$ are given by (51) and (53).

The two evolution laws are based on Griffith's crucial assumption [1921] concerning the surface energy associated with a crack. Specifically, assume that there exists a material constant $G_{c}>0$ such that the surface energy of the body with a crack of length $l$ is

$$
\mathscr{S}(l)=\mathrm{G}_{\mathrm{c}} l .
$$



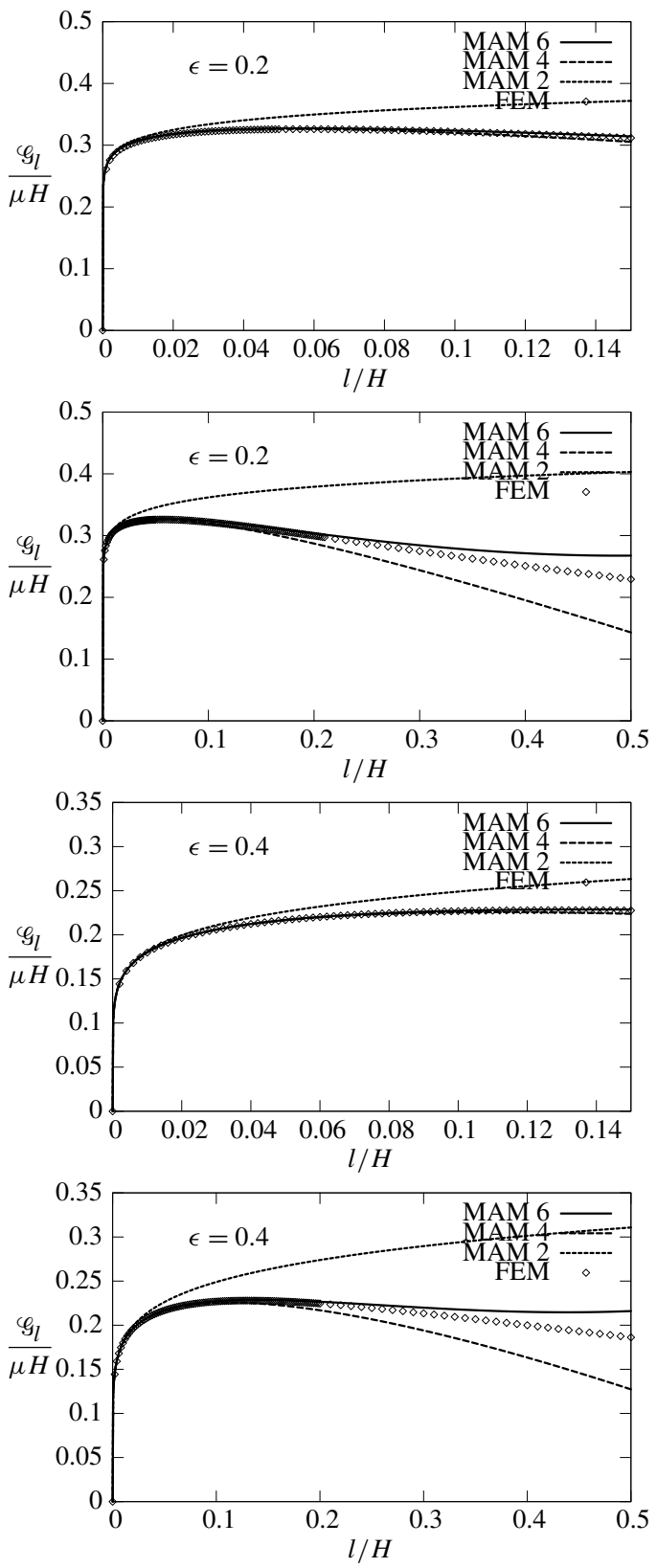

Figure 7. Comparison of the graphs of $\varphi_{l}$ obtained by the MAM and by COMSOL for $\epsilon=0.2$ (top two plots) and $\epsilon=0.4$ (bottom two). The curve labeled FEM indicates points obtained by COMSOL, while the curves labeled MAM $2 i, i \in\{1,2,3\}$, indicate that the first $i$ nontrivial terms in the expansion of $\mathscr{G}_{l}$ were considered. 
Accordingly, the total energy of the body at equilibrium at time $t$ with a crack of length $l$ becomes

$$
\mathscr{E}(t, l):=\mathscr{P}(t, l)+\mathscr{S}(l)=t^{2} \mathscr{P}_{l}+\mathrm{G}_{c} l .
$$

Throughout this section we assume that $l \mapsto \mathscr{P}_{l}$ is continuously differentiable and monotonically decreasing. Moreover, some monotonic properties of $l \mapsto \varphi_{l}$ will be added when necessary according to the analysis made in the previous sections.

4.1. The two evolution laws. Let us briefly introduce the two evolution laws; the reader interested in the details should refer to [Marigo 2010]. The first one, called the G-law, is the usual Griffith law based on the critical potential energy release rate criterion; see [Bui 1978; Leblond 2003; Nguyen 2000]. In essence, this law only investigates smooth (i.e., at least continuous) evolutions of the crack length with the loading. It consists of the three following items:

Definition $2\left(G\right.$-law). Let $l_{0} \in[0, L]$. A continuous function $t \mapsto l(t)$ is said to satisfy (or to be a solution of) the $G$-law in the interval $\left[t_{0}, t_{1}\right]$ with the initial condition $l\left(t_{0}\right)=l_{0}$, if the three following properties hold:

(1) Irreversibility: $t \mapsto l(t)$ is not decreasing;

(2) Energy release rate criterion: $\mathscr{G}(t, l(t)) \leq \mathrm{G}_{\mathrm{c}}$ for all $t \in\left[t_{0}, t_{1}\right]$;

(3) Energy balance: $l(t)$ is increasing only if $\mathscr{G}(t, l(t))=\mathrm{G}_{\mathrm{c}}$; i.e., if $\mathscr{G}(t, l(t))<$ $\mathrm{G}_{\mathrm{c}}$ at some $t$, then $l\left(t^{\prime}\right)=l(t)$ for every $t^{\prime}$ in a certain neighborhood $[t, t+h)$ of $t$.

The third item implies that the release of potential energy is equal to the created surface energy when the crack propagates, which justifies its name "energy balance". Consequently, if $t \mapsto l(t)$ is absolutely continuous, then the third item is equivalent to

$$
\frac{\partial \mathscr{E}}{\partial l}(t, l(t)) \dot{l}(t)=0
$$

for almost all $t$, and the following equality holds for almost all $t$ :

$$
\frac{d}{d t} \mathscr{E}(t, l(t))=\frac{\partial \mathscr{E}}{\partial t}(t, l(t)) .
$$

A major drawback of the G-law is the inability to take into account discontinuous crack evolutions, which renders it useless in many situations as we will see in the next subsection. It must be replaced by another law which admits discontinuous solutions. Another motivation of changing the G-law is to reinforce the second item by introducing a full stability criterion; see [Francfort and Marigo 1998; Nguyen 2000; Bourdin et al. 2008]. Specifically, let us consider the local stability condition

$$
\forall t \geq 0, \exists h(t)>0: \quad \mathscr{E}(t, l(t)) \leq \mathscr{E}(t, l) \quad \forall l \in[l(t), l(t)+h(t)],
$$


which requires that the total energy at $t$ is a "unilateral" local minimum. (The qualifier unilateral is added because the irreversibility condition leads to comparing the energy at $t$ with only that corresponding to greater crack length; see [Bourdin et al. 2008].) Taking $l=l(t)+h$ with $h>0$ in (67), dividing by $h$ and passing to the limit when $h \rightarrow 0$, we recover the critical energy release rate criterion. Thus, the second item can be seen as a first-order stability condition, weaker than (67). A stronger requirement is obtained by replacing local minimality by global minimality. It was the condition introduced in [Francfort and Marigo 1998] in the spirit of the original Griffith idea [1921], and we will adopt it here.

Definition 3 (FM-law). A function $t \mapsto l(t)$ (defined for $t \geq 0$ and with values in $[0, L]$ ) is said to satisfy (or to be a solution of) the FM-law if the three following properties hold:

(1) Irreversibility: $t \mapsto l(t)$ is not decreasing;

(2) Global stability: $\mathscr{E}(t, l(t)) \leq \mathscr{E}(t, l)$ for all $t \geq 0$ and all $l \in[l(t), L]$;

(3) Energy balance: $\mathscr{E}(t, l(t))=\mathscr{E}\left(0, l_{0}\right)+\int_{0}^{t} \partial \mathscr{E} / \partial t^{\prime}\left(t^{\prime}, l\left(t^{\prime}\right)\right) d t^{\prime}$ for all $t \geq 0$.

Let us note that the irreversibility condition is unchanged, while the energy balance condition is now written as the integrated form of (66), which does not require that $t \mapsto l(t)$ be continuous. Note also that the energy balance implies $l(0)=l_{0}$ because $0=\mathscr{E}(0, l(0))-\mathscr{E}\left(0, l_{0}\right)=\mathrm{G}_{\mathrm{c}}\left(l(0)-l_{0}\right)$, and that the second item is automatically satisfied at $t=0$ because $\mathscr{E}(0, l)=\mathrm{G}_{c} l$.

4.2. The main properties of the G-law and the FM-law. We recall or establish in this subsection some results for the two evolution laws under the assumptions of monotonicity of $l \mapsto \varphi_{l}$ resulting from the numerical computations; see (P1)(P4) in Section 3.3. Some of those results have a general character and have been previously established in [Bourdin et al. 2008; Francfort and Marigo 1998; Marigo 2010], while the other ones are specific to the present problem. In the case of properties which have already been obtained, we simply recall them without proofs.

Let us first consider the case when the notch is in fact a crack. Then, the two laws are equivalent by virtue of:

Proposition 5. In the case $\epsilon=0$, since $l \mapsto \mathscr{G}_{l}$ is decreasing from $\mathscr{G}_{0}>0$ to 0 when $l$ goes from 0 to $L$ (see property $(\mathbf{P 1})$ ), the G-law and the FM-law admit the same unique solution. Specifically, the preexisting crack begins to propagate at time $\mathrm{t}_{\mathrm{i}}$ such that $\mathrm{t}_{\mathrm{i}}{ }^{2} \varphi_{l_{0}}=\mathrm{G}_{\mathrm{c}}$. Then the crack propagates continuously and $l(t)$ is such that $t^{2} \varphi_{l(t)}=G_{c}$. Since $\varphi_{L}=0$, the crack will not reach the end $L$ in a finite time.

Proof. See [Marigo 2010, Proposition 18].

In the case of a genuine notch, as far as the nucleation and the propagation of a crack with the G-law are concerned, we have: 
Proposition 6. In the case $\epsilon>0$, according to $l_{0}=0$ or $l_{0} \in\left(0, l_{\mathrm{m}}\right)$ or $l_{0} \in\left[l_{\mathrm{m}}, L\right)$, the crack evolution predicted by the G-law is as follows:

(1) If $l_{0}=0$, since $\mathscr{G}_{0}=0$, the unique solution to the G-law is $l(t)=0$ for all $t$; i.e., there is no crack nucleation.

(2) If $l_{0} \in\left(0, l_{\mathrm{m}}\right)$, then the preexisting crack begins to propagate at time $\mathrm{t}_{\mathrm{i}}$ such that $\mathrm{t}_{\mathrm{i}}^{2} \varphi_{l_{0}}=\mathrm{G}_{\mathrm{c}}$. But at $\mathrm{t}_{\mathrm{i}}$ the propagation is necessarily discontinuous and hence there is no continuous solution to the G-law for $t \geq \mathrm{t}_{\mathrm{i}}$.

(3) If $l_{0} \in\left[l_{\mathrm{m}}, L\right)$, since $l \mapsto \mathscr{G}_{l}$ is monotonically decreasing in the interval $\left(l_{\mathrm{m}}, L\right)$, the situation is the same as in Proposition 5. There exists a unique solution for the G-law: the crack begins to propagate at $\mathrm{t}_{\mathrm{i}}$ (still given by $\mathrm{t}_{\mathrm{i}}^{2} \varphi_{l_{0}}=\mathrm{G}_{\mathrm{c}}$ ) and then propagates continuously until L, which is reached asymptotically.

Proof. Let us give the sketch of the proof for the first two items.

(1) Since $l_{0}=0$ and $\mathscr{G}_{0}=0$, then for all $t \geq 0$ one gets $0=\mathscr{G}(t, 0)<\mathrm{G}_{\mathrm{c}}$ and hence $l(t)=0$ is a solution. The uniqueness follows from the initial condition and the energy balance.

(2) Since $0<l_{0}<l_{\mathrm{m}}$, then $\mathscr{G}_{l_{0}}>0$ and hence $t^{2} \mathscr{G}_{l_{0}}=\mathscr{G}\left(t, l_{0}\right) \leq \mathrm{G}_{\mathrm{c}}$ if and only if $t \in\left[0, \mathrm{t}_{\mathrm{i}}\right]$. Since the inequality is strict when $t \in\left[0, \mathrm{t}_{\mathrm{i}}\right)$, then $l(t)=0$ is the unique solution in this interval because of the initial condition and the energy balance. By continuity, it is also the unique solution in the closed interval $\left[0, \mathrm{t}_{\mathrm{i}}\right]$. On the other hand, since $\mathscr{G}\left(t, l_{0}\right)>\mathrm{G}_{\mathrm{c}}$ when $t>\mathrm{t}_{\mathrm{i}}$, the crack must begin to propagate at $t_{i}$.

Let us show that no (continuous) evolution can satisfy the G-law for $t>\mathrm{t}_{\mathrm{i}}$. Indeed, by construction $\mathscr{G}\left(\mathrm{t}_{\mathrm{i}}, l\left(\mathrm{t}_{\mathrm{i}}\right)\right)=\mathrm{t}_{\mathrm{i}}^{2} \mathscr{G}_{l_{0}}=\mathrm{G}_{\mathrm{c}}$. But since $l(t) \geq l_{\mathrm{i}}$ for $t>\mathrm{t}_{\mathrm{i}}$ and since $l \mapsto \mathscr{G}_{l}$ is monotonically increasing in the neighborhood of $l_{0}<l_{\mathrm{m}}$, we have for $t \in\left(\mathrm{t}_{\mathrm{i}}, \mathrm{t}_{\mathrm{i}}+h\right)$ and a sufficiently small $h>0$ :

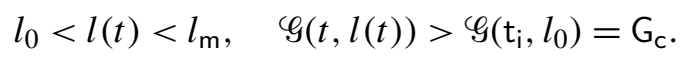

Therefore the energy release rate criterion cannot be satisfied by a continuous evolution in a neighborhood of $t_{i}$. The unique possibility is that the length of the crack jumps from $l_{0}$ to some $l_{\mathrm{i}}>l_{\mathrm{m}}$ at time $\mathrm{t}_{\mathrm{i}}$. But that requires reformulating the G-law.

The proof of the third item is the same as in the previous proposition and hence we refer to [Marigo 2010, Proposition 18].

Remark 8. This property of no nucleation of a crack at a notch or of brutal propagation of a short crack is due to the fact that a notch with Neumann boundary conditions induces a weak singularity only; i.e., $\lambda>1 / 2$. If one changes the boundary conditions by imposing the displacement on one edge of the notch and the stress on the other edge, then the singularity becomes strong for $\omega$ large enough 
and in such a case all the properties of nucleation are changed; see [Francfort and Marigo 1998, Proposition 4.19].

Consider now the FM-law. It is proved in [Marigo 2010, Proposition 3] that, in the case of a monotonically increasing loading, the FM-law is equivalent to a minimization problem of the total energy at each time, as precisely stated in the following lemma:

Lemma 7. Let $l_{0} \in[0, L)$ be the initial length of the crack. A function $t \mapsto l(t)$ satisfies the FM-law if and only if, at each $t, l(t)$ is a minimizer of $l \mapsto \mathscr{E}(t, l)$ over $\left[l_{0}, L\right]$. Therefore, the FM-law admits at least one solution and each solution grows from $l_{0}$ to $L$.

This property holds true for any $\epsilon \geq 0$. In the case $\epsilon>0$ we can deduce precise results:

Proposition 8. In the case $\epsilon>0$, according to $l_{0} \in\left[0, l_{\mathrm{m}}\right)$ or $l_{0} \in\left[l_{\mathrm{m}}, L\right)$, the crack evolution predicted by the FM-law is as follows:

(1) If $l_{0} \in\left[0, l_{\mathrm{m}}\right.$ ), then the nucleation (if $l_{0}=0$ ) or the propagation of the preexisting crack (if $\left.l_{0} \neq 0\right)$ starts at time $\mathrm{t}_{\mathrm{i}}>0$ and at this time the crack length jumps instantaneously from $l_{0}$ to $l_{\mathrm{i}}$. The length $l_{\mathrm{i}}$ is the unique length in $\left(l_{\mathrm{m}}, L\right)$ such that

$\int_{l_{0}}^{l_{\mathrm{i}}} \mathscr{G}_{l} d l=\left(l_{\mathrm{i}}-l_{0}\right) \mathscr{G}_{l_{\mathrm{i}}}, \quad$ or, equivalently, $\quad \mathscr{P}_{l_{0}}-\mathscr{P}_{l_{\mathrm{i}}}=\left(l_{\mathrm{i}}-l_{0}\right) \mathscr{G}_{l_{\mathrm{i}}}$,

while the time $\mathrm{t}_{\mathrm{i}}$ is given by

$$
\mathrm{t}_{\mathrm{i}}^{2} \varphi_{l_{\mathrm{i}}}=\mathrm{G}_{\mathrm{c}} .
$$

After this jump, the crack propagates continuously from $l_{\mathrm{i}}$ to $L$, the evolution satisfying then the G-law; i.e.,

$$
t^{2} \varphi_{l(t)}=\mathrm{G}_{\mathrm{c}} \text { for all } t>\mathrm{t}_{\mathrm{i}} .
$$

(2) If $l_{0} \in\left[l_{\mathrm{m}}, L\right)$, since $l \mapsto \mathscr{G}_{l}$ is monotonically decreasing in the interval $\left(l_{\mathrm{m}}, L\right)$, the situation is the same as in Proposition 5. There exists a unique solution for the FM-law which is the same as for the G-law: the crack begins to propagate at $\mathrm{t}_{\mathrm{i}}$ such that $\mathrm{t}_{\mathrm{i}}^{2} \varphi_{l_{0}}=\mathrm{G}_{\mathrm{c}}$ and then propagates continuously until L, which is reached asymptotically.

Remark 9. Before the proof of this proposition, let us comment and interpret (68) giving the jump of the crack at $t_{i}$. 


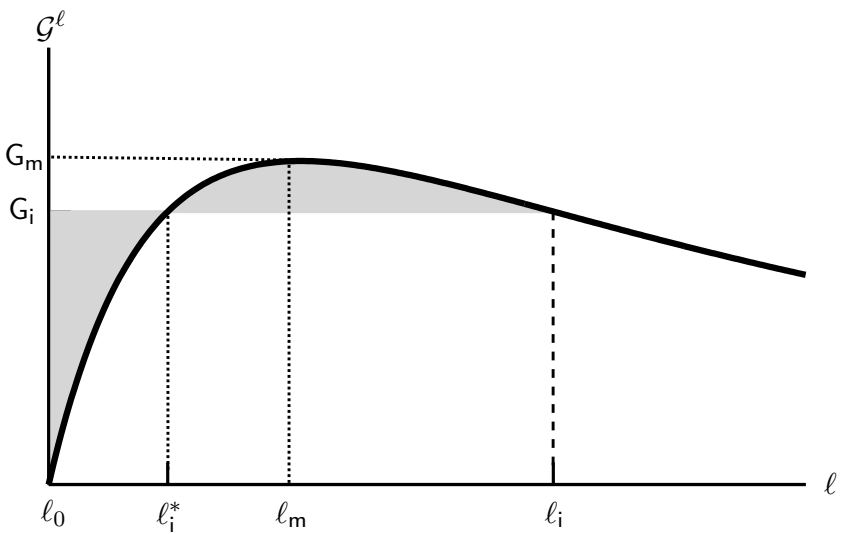

Figure 8. Graphical interpretation of the criterion of crack nucleation given by the FM-law and which obeys the Maxwell rule of equal areas.

- Let us first prove that $l_{\mathrm{i}}$ is well defined by (68). Let $l \mapsto g(l)$ be the function defined for $l \in\left(l_{\mathrm{m}}, L\right)$ by

$$
g(l)=\int_{l_{0}}^{l} \varphi_{l} d l-\left(l-l_{0}\right) \mathscr{G}_{l} .
$$

Its derivative is given by $g^{\prime}(l)=-\left(l-l_{0}\right) \mathscr{G}_{l}^{\prime}$ and hence is positive because $\mathscr{G}_{l}$ is decreasing in $\left(l_{\mathrm{m}}, L\right)$. Since $\varphi_{l}<\mathrm{G}_{\mathrm{m}}:=\mathscr{G}_{l_{\mathrm{m}}}, g\left(l_{\mathrm{m}}\right)<0$, whereas $g(L)>0$ because $\varphi_{L}=0$. Therefore, there exists a unique $l \in\left(l_{\mathrm{m}}, L\right)$ such that $g(l)=0$, what is precisely the definition of $l_{\mathrm{i}}$.

- Equation (68) giving $l_{\mathrm{i}}$ has a graphical interpretation. Indeed, the integral over $\left(l_{0}, l_{\mathrm{i}}\right)$ represents the area under the graph of $l \mapsto \mathscr{G}_{l}$ between the lengths $l_{0}$ and $l_{\mathrm{i}}$. On the other hand the product $\left(l_{\mathrm{i}}-l_{0}\right) \mathscr{G}_{l_{\mathrm{i}}}$ represents the area of the rectangle whose height is $G_{i}:=\mathscr{G}_{l_{\mathrm{i}}}$. Therefore, since these two areas are equal, the two gray areas of Figure 8 are also equal. This rule of equality of the areas determines $l_{\mathrm{i}}$ and, by essence, the line $G=\mathrm{G}_{\mathrm{i}}$ is the classical Maxwell line which appears in any problem of minimization of a nonconvex function.

- Note that $l_{\mathrm{i}}$ is independent of the toughness $\mathrm{G}_{\mathrm{c}}$ and of the shear modulus $\mu$ of the material. It is a characteristic of the structure and merely depends on the geometry and the type of loading. Here, it depends on $\epsilon, H$ and $L$. For a given $\epsilon$ and a given ratio $L / H, l_{\mathrm{i}}$ is proportional to $H, l_{\mathrm{i}}=\tilde{l}_{\mathrm{i}} H$. This property is a consequence of the Griffith assumption on the surface energy.

- The critical loading amplitude $\mathrm{t}_{\mathrm{i}}$ depends on the toughness and on the size of the body. Since $\mathscr{G}_{l_{\mathrm{i}}}=\tilde{\varphi}_{l_{\mathrm{i}}} \mu H, \mathrm{t}_{\mathrm{i}}$ varies like $1 / \sqrt{H}$. This size effect is also a consequence of the Griffith assumption on the surface energy. 
- By virtue of (68) and (69), the energy balance holds at time $t_{\mathrm{i}}$ even if the crack jumps at this time; i.e., the total energy of the body just before the jump is equal to the total energy just after. Indeed, those energies are respectively given by

$$
\mathscr{E}\left(\mathrm{t}_{\mathrm{i}}-, l_{0}\right)=\mathrm{t}_{\mathrm{i}}^{2} \mathscr{P}_{l_{0}}+\mathrm{G}_{\mathrm{c}} l_{0}, \quad \mathscr{E}\left(\mathrm{t}_{\mathrm{i}}+, l_{\mathrm{i}}\right)=\mathrm{t}_{\mathrm{i}}^{2} \mathscr{P}_{l_{\mathrm{i}}}+\mathrm{G}_{\mathrm{c}} l_{\mathrm{i}} .
$$

Using (68), (69) and the equality $\mathscr{P}_{l_{0}}-\mathscr{P}_{l_{\mathrm{i}}}=\int_{l_{0}}^{l_{\mathrm{i}}} \varphi_{l} d l$, then $\mathscr{E}\left(\mathrm{t}_{\mathrm{i}}-, l_{0}\right)=$ $\mathscr{E}\left(\mathrm{t}_{\mathrm{i}}+, l_{\mathrm{i}}\right)$.

Proof of Proposition 8. We just prove the first part of the proposition and the reader should refer to [Marigo 2010, Proposition 18] for the proof of the second part. Let $l_{0} \in\left[0, l_{\mathrm{m}}\right)$. By virtue of Lemma $7, l(t)$ is a minimizer of $l \mapsto \mathscr{E}(t, l)$ over $\left[l_{0}, L\right]$. (The minimum exists because the energy is continuous and the interval is compact.) Let $l_{\mathrm{i}}, \mathrm{t}_{\mathrm{i}}$ be given by (68)-(69), let $\mathrm{G}_{\mathrm{i}}=\mathscr{G}_{l_{\mathrm{i}}}$ and let $l_{\mathrm{i}}^{*}$ be the other length such that $\varphi_{l_{i}^{*}}=\mathrm{G}_{\mathrm{i}}$; see Figure 8. Let us first remark that the function $l \mapsto \bar{g}(l)$ defined on $\left[l_{0}, L\right]$ by

$$
\bar{g}(l):=\mathrm{G}_{\mathrm{i}}\left(l-l_{0}\right)-\left(\mathscr{P}_{l_{0}}-\mathscr{P}_{l}\right)
$$

is nonnegative and vanishes only at $l_{0}$ and $l_{\mathrm{i}}$. Indeed, its derivative is $\bar{g}^{\prime}(l)=\mathrm{G}_{\mathrm{i}}-\varphi_{l}$. Hence, $\bar{g}$ is first increasing from 0 when $l$ grows from $l_{0}$ to $l_{\mathrm{i}}^{*}$, then decreasing to 0 when $l$ grows from $l_{\mathrm{i}}^{*}$ to $l_{\mathrm{i}}$, and finally increasing again from 0 when $l$ grows from $l_{\mathrm{i}}$ to $L$.

Let us show that $l_{0}$ is the unique minimizer of the total energy when $t<\mathrm{t}_{\mathrm{i}}$. From (68) and (69), we get for all $l \in\left[l_{0}, L\right]$ and all $t \leq \mathrm{t}_{\mathrm{i}}$ :

$$
\mathscr{E}(t, l)-\mathscr{E}\left(t, l_{0}\right)=-t^{2}\left(\mathscr{P}_{l_{0}}-\mathscr{P}_{l}\right)+\mathrm{G}_{\mathrm{c}}\left(l-l_{0}\right) \geq t^{2} \bar{g}(l) \geq 0 .
$$

Moreover, the inequalities above are equalities if and only if $l=l_{0}$ when $t<\mathrm{t}_{\mathrm{i}}$ and the result follows. Using the same estimates, we can deduce that $l_{0}$ and $l_{\mathrm{i}}$ are the two minimizers of the total energy at $t=\mathrm{t}_{\mathrm{i}}$.

Let us show now that the minimizer is in the open interval $\left(l_{\mathrm{i}}, L\right)$ when $t>\mathrm{t}_{\mathrm{i}}$. From (68) and (69), we get for all $l \in\left[l_{0}, l_{\mathrm{i}}\right.$ ) and all $t>\mathrm{t}_{\mathrm{i}}$ :

$$
\begin{aligned}
\mathscr{E}(t, l)-\mathscr{E}\left(t, l_{\mathrm{i}}\right) & =t^{2}\left(\mathscr{P}_{l}-\mathscr{P}_{l_{\mathrm{i}}}\right)-\mathrm{G}_{\mathrm{c}}\left(l_{\mathrm{i}}-l\right)>\mathrm{t}_{\mathrm{i}}{ }^{2}\left(\mathscr{P}_{l}-\mathscr{P}_{l_{\mathrm{i}}}-\mathrm{G}_{\mathrm{i}}\left(l_{\mathrm{i}}-l\right)\right) \\
& =\mathrm{t}_{\mathrm{i}}{ }^{2}\left(\bar{g}(l)-\bar{g}\left(l_{\mathrm{i}}\right)\right)=\mathrm{t}_{\mathrm{i}}{ }^{2} \bar{g}(l) \geq 0 .
\end{aligned}
$$

Hence, the minimizer cannot be in $\left[l_{0}, l_{\mathrm{i}}\right)$. Since the derivative of the total energy at $l=l_{\mathrm{i}}$ is equal to $\mathrm{G}_{\mathrm{c}}-t^{2} \mathrm{G}_{\mathrm{i}}<0, l_{\mathrm{i}}$ is not the minimizer. In the same manner, since the derivative of the total energy at $l=L$ is equal to $\mathrm{G}_{\mathrm{c}}-t^{2} \varphi_{L}=\mathrm{G}_{\mathrm{c}}>0, L$ cannot be the minimizer. Therefore, the minimizer is in the interval $\left(l_{\mathrm{i}}, L\right)$ when $t>\mathrm{t}_{\mathrm{i}}$. Hence, it must be such that the derivative of the total energy vanishes, which yields $t^{2} \varphi_{l(t)}=\mathrm{G}_{\mathrm{c}}$. Since $l \mapsto \mathscr{G}_{l}$ is monotonically decreasing from $\mathrm{G}_{\mathrm{i}}$ to 0 when $l$ goes 
from $l_{\mathrm{i}}$ to $L$, there exists a unique $l(t) \in\left(l_{\mathrm{i}}, L\right)$ such that $\varphi_{l(t)}=\mathrm{G}_{\mathrm{c}} / t^{2}<\mathrm{G}_{\mathrm{i}}$. The proof of the first part is complete.

4.3. Computation of the crack nucleation by the MAM. Let us consider the cases where $\epsilon$ is sufficiently small in order that $l \mapsto \mathscr{G}_{l}$ be well approximated by the first two nontrivial terms of its matched asymptotic expansion for $l$ in the interval $\left[0,2 l_{\mathrm{m}}\right]$; see $(\mathbf{C 4})$. Accordingly, we have

$$
\frac{\mathscr{G}_{l}}{\mu H} \approx 2 \lambda\left|P_{2}\right|\left(\frac{l}{H}\right)^{2 \lambda-1}-4 \lambda\left|P_{4}\right|\left(\frac{l}{H}\right)^{4 \lambda-1},
$$

using the fact that $P_{2}<0$ and $P_{4}>0$. Therefore, the length $l_{\mathrm{m}}$ where $\varphi_{l}$ is maximal and the maximum $G_{m}$ are approximated by

$$
\frac{l_{\mathrm{m}}}{H} \approx\left(\frac{(2 \lambda-1)\left|P_{2}\right|}{2(4 \lambda-1)\left|P_{4}\right|}\right)^{\frac{1}{2 \lambda}}, \quad \frac{\mathrm{G}_{\mathrm{m}}}{\mu H} \approx \frac{4 \lambda^{2}\left|P_{2}\right|}{4 \lambda-1}\left(\frac{(2 \lambda-1)\left|P_{2}\right|}{2(4 \lambda-1)\left|P_{4}\right|}\right)^{\frac{2 \lambda-1}{2 \lambda}} .
$$

Comparing with the values obtained by the FEM (see (P3) and Table 5), it appears that the agreement is very good for the maximum $\mathrm{G}_{\mathrm{m}}$, and less good for $l_{\mathrm{m}}$. The reason is that the localization of $l_{\mathrm{m}}$ by the FEM is quite imprecise because the graph of $\varphi_{l}$ is very flat near $l_{\mathrm{m}}$ : for instance, for $\epsilon=0.3, \mathscr{G}_{l}$ computed at $\tilde{l}=0.092$ is equal to 0.27327 while it is equal to 0.27307 at $\tilde{l}=0.082$, with a relative difference less than $10^{-4}$.

One can see also in Table 5 that the contribution of the next term, i.e., MAM 6 , is weak when $\epsilon$ is less than 0.2. Its influence, in particular on $l_{\mathrm{i}}$, can no longer be neglected when $\epsilon \geq 0.3$. Note also that MAM 4 underestimates while MAM 6 overestimates the lengths $l_{\mathrm{m}}$ and $l_{\mathrm{i}}$. This bounding property is due to the alternating of the sign of the coefficients $P_{2 i}$ with $i$. However, it is checked numerically only; we are not able to prove it. Using MAM 4 to calculate the nucleation, we obtain the following result:

Proposition 9. In the case of a genuine notch $\epsilon>0$ :

(1) If the body does not contain a preexisting crack $\left(l_{0}=0\right)$, then the time $\mathrm{t}_{\mathrm{i}}$ at which the crack nucleates and the length $l_{\mathrm{i}}$ of the nucleated crack at this time are approximated with the MAM 4 by

$$
\begin{aligned}
& \frac{l_{\mathrm{i}}}{H} \approx 2^{\frac{1}{2 \lambda}} \frac{l_{\mathrm{m}}}{H} \approx\left(\frac{(2 \lambda-1)\left|P_{2}\right|}{(4 \lambda-1)\left|P_{4}\right|}\right)^{\frac{1}{2 \lambda}}, \\
& \mathrm{t}_{\mathrm{i}}{ }^{2} \approx \frac{1}{\lambda 2^{\frac{1}{2 \lambda}}} \frac{\mathrm{G}_{\mathrm{c}}}{\mathrm{G}_{\mathrm{m}}} \approx \frac{\mathrm{t}_{\mathrm{c}}{ }^{2}}{8 \lambda^{3}}\left(\frac{4 \lambda-1}{\left|P_{2}\right|}\right)^{2-\frac{1}{2 \lambda}}\left(\frac{4 P_{4}}{2 \lambda-1}\right)^{1-\frac{1}{2 \lambda}},
\end{aligned}
$$

where $\mathrm{t}_{\mathrm{c}}{ }^{2}=\mathrm{G}_{\mathrm{c}} / \mu H$. 


\begin{tabular}{|c|ccccc|}
\hline$\epsilon$ & 0 & 0.1 & 0.2 & 0.3 & 0.4 \\
$\lambda$ & 0.5 & 0.5164 & 0.5335 & 0.5511 & 0.5689 \\
\hline$l_{\mathrm{m}} / H$ by FEM & 0 & 0.024 & 0.058 & 0.092 & 0.130 \\
$l_{\mathrm{m}} / H$ by MAM 4 & 0 & 0.0255 & 0.0533 & 0.0823 & 0.1124 \\
$l_{\mathrm{m}} / H$ by MAM 6 & 0 & 0.0267 & 0.0584 & 0.0953 & 0.1387 \\
$\mathrm{G}_{\mathrm{m}} / \mu H$ by FEM & 0.4820 & 0.3900 & 0.3260 & 0.2733 & 0.2279 \\
$\mathrm{G}_{\mathrm{m}} / \mu H$ by MAM 4 & 0.4820 & 0.3917 & 0.3264 & 0.2724 & 0.2257 \\
$\mathrm{G}_{\mathrm{m}} / \mu H$ by MAM 6 & 0.4820 & 0.3917 & 0.3274 & 0.2743 & 0.2287 \\
$l_{\mathrm{i}} / H$ by FEM & 0 & 0.0517 & 0.1131 & 0.1814 & 0.2561 \\
$l_{\mathrm{i}} / H$ by MAM 4 & 0 & 0.0499 & 0.1020 & 0.1544 & 0.2067 \\
$l_{\mathrm{i}} / H$ by MAM 6 & 0 & 0.0530 & 0.1163 & 0.1923 & 0.2964 \\
$\mathrm{G}_{\mathrm{i}} / \mu H$ by FEM & 0.4820 & 0.3864 & 0.3195 & 0.2650 & 0.2188 \\
$\mathrm{G}_{\mathrm{i}} / \mu H$ by MAM 4 & 0.4820 & 0.3877 & 0.3195 & 0.2635 & 0.2157 \\
$\mathrm{G}_{\mathrm{i}} / \mu H$ by MAM 6 & 0.4820 & 0.3881 & 0.3208 & 0.2662 & 0.2201 \\
$\mathrm{t}_{\mathrm{i}} / \mathrm{t}_{\mathrm{c}}$ by FEM & 1.440 & 1.605 & 1.766 & 1.938 & 2.132 \\
$\mathrm{t}_{\mathrm{i}} / \mathrm{t}_{\mathrm{c}}$ by MAM 4 & 1.440 & 1.606 & 1.769 & 1.916 & 2.153 \\
$\mathrm{t}_{\mathrm{i}} / \mathrm{t}_{\mathrm{c}}$ by MAM 6 & 1.440 & 1.605 & 1.766 & 1.938 & 2.131 \\
\hline
\end{tabular}

Table 5. Comparisons of the values of $l_{\mathrm{m}}, \mathrm{G}_{\mathrm{m}}, l_{\mathrm{i}}, \mathrm{G}_{\mathrm{i}}$ and $\mathrm{t}_{\mathrm{i}}$ obtained by the FEM with those obtained by MAM 4 and MAM 6 .

(2) If the body contains a preexisting crack of length $l_{0}$ such that $0<l_{0}<l_{\mathrm{m}}$, then the length $l_{\mathrm{i}}$ at which the crack jumps at the onset of the propagation is the unique solution greater than $l_{\mathrm{m}}$ of

$$
\begin{aligned}
0=\left|P_{2}\right|\left((2 \lambda-1) l_{\mathrm{i}}^{2 \lambda}-2 \lambda l_{0} l_{\mathrm{i}}^{2 \lambda-1}\right. & \left.+l_{0}^{2 \lambda}\right) H^{2 \lambda} \\
& -P_{4}\left((4 \lambda-1) l_{\mathrm{i}}^{4 \lambda}+4 \lambda l_{0} l_{\mathrm{i}}^{4 \lambda-1}-l_{0}^{4 \lambda}\right),
\end{aligned}
$$

while the time $\mathrm{t}_{\mathrm{i}}$ at which the onset occurs is given by $\mathrm{t}_{\mathrm{i}}^{2}=\mathrm{G}_{\mathrm{c}} / \mathscr{G}_{l_{\mathrm{i}}}$. Therefore, $l_{\mathrm{i}}$ and $\mathrm{t}_{\mathrm{i}}$ decrease from the values given by (72) to $l_{\mathrm{m}}$ and $\sqrt{\mathrm{G}_{\mathrm{c}} / \mathrm{G}_{\mathrm{m}}}$ given by (71) when $l_{0}$ runs from 0 to $l_{\mathrm{m}}$.

Proof. When $l_{0}=0$, using MAM 4, then (68) becomes

$$
0=(2 \lambda-1)\left|P_{2}\right|\left(\frac{l_{\mathrm{i}}}{H}\right)^{2 \lambda}-(4 \lambda-1)\left|P_{4}\right|\left(\frac{l_{\mathrm{i}}}{H}\right)^{4 \lambda} .
$$

Using (71), (72) can be deduced after some calculations left to the reader. In the same manner, (73) is a direct consequence of (68) and (70). The monotonicity of $l_{\mathrm{i}}$ and $t_{i}$ with respect to $l_{0}$ is easily checked from the graphical interpretation of (73); see Figure 8. 


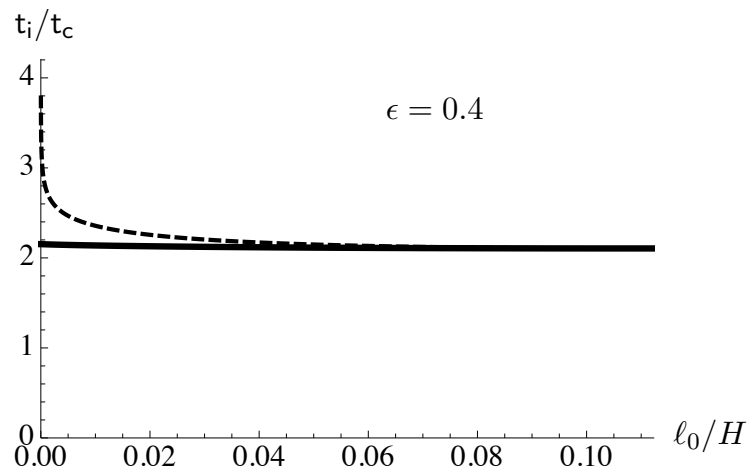

Figure 9. Time at which a preexisting crack starts as a function of its length in the case where the notch parameter $\epsilon$ equals 0.4 . Plain line: from the FM-law; dashed line: from the G-law.

Therefore, since $1 / 2<\lambda<1$ for a genuine notch, the length of the nucleated crack $l_{\mathrm{i}}$ is less than $2 l_{\mathrm{m}}$ while the critical time $\mathrm{t}_{\mathrm{i}}$ is not greater than $2^{1 / 4} \sqrt{\mathrm{G}_{\mathrm{c}} / \mathrm{G}_{\mathrm{m}}}$. For a very sharp notch, i.e., when $\epsilon$ is small, then $2 \lambda \approx 1+\epsilon / \pi$ and

$$
l_{\mathrm{i}} \approx \frac{\epsilon\left|P_{2}\right|}{\pi P_{4}} H, \quad \mathrm{t}_{\mathrm{i}}^{2} \approx \frac{\mathrm{G}_{\mathrm{c}}}{\left|P_{2}\right| \mu H},
$$

where $P_{2} \approx-0.4820$ and $P_{4} \approx 0.3282$. Therefore we recover the response associated with a crack when the notch angle tends to $2 \pi$. The FM-law delivers an evolution which depends continuously on the parameter $\epsilon$, in contrast with the G-law.

As long as the dependence of $t_{i}$ on $l_{0}$ is concerned, it turns out that the FM-law predicts that the variation of $\mathrm{t}_{\mathrm{i}}$ is small when $l_{0}$ goes from 0 to $l_{\mathrm{m}}$ as can be seen in Figure 9 for $\epsilon=0.4$. Indeed, $\mathrm{t}_{\mathrm{i}} / \mathrm{t}_{\mathrm{c}}$ decreases from 2.153 to 2.105 when $l_{0}$ varies from 0 to $l_{\mathrm{m}}=0.112 \mathrm{H}$. This constitutes also a strong difference with the prediction of the $G$-law for which $t_{i}$ goes to infinity when $l_{0}$ goes to 0 .

\section{Conclusion and perspectives}

We have presented here a general method based on matched asymptotic expansions which can be applied to determine the mechanical fields and all related mechanical quantities in the case of a defect located at the tip of a notch. Applying this method to the case of a noncohesive crack, it turns out that it is sufficient to solve a few inner and outer problems to compute with very good accuracy the dependency of the energy and the energy release rate on the length of the crack. Moreover, this approximation can be used for very small values of the length of the crack and hence to determine the onset of the cracking, whereas a classical finite element 
method gives rise to inaccurate results. In particular, the matched asymptotic method permits a comparison of the nucleation process of a crack at the tip of the notch which is predicted by the classical Griffith criterion with that predicted by the principle of energy minimization proposed in [Francfort and Marigo 1998]. It turns out that the latter principle gives rise to much more relevant results than the former, from a physical viewpoint.

A natural extension of this work is to consider situations where the geometry and the loading have no symmetry and hence the direction that the nucleated crack will choose must also be predicted. Let us note that the G-law alone is not able to give an answer, and another criterion must be supplemented. In an antiplane setting, the principle of local symmetry, which is by essence made for an isotropic plane setting, cannot be used. It turns out that the FM-law in its general statement can also predict the direction and more generally the path of the crack; see [Chambolle et al. 2009; 2010; Francfort and Marigo 1998]. So, an interesting challenge should be to use the MAM and the FM-law in a nonsymmetric case to predict also the direction of nucleation. Another natural and desirable extension of the present work is to develop the method in a plane elasticity setting. It seems that there is no conceptual difficulty in doing that. The last perspective concerns the choice of the surface energy. Indeed, the present study is based on the crucial Griffith assumption that the surface energy is proportional to the crack area. This assumption has very important consequences on the nucleation as we have seen in the paper. With this hypothesis, there is no cohesive force and hence the model does not contain the concept of critical stress. An important step will be to apply the MAM in the case of a cohesive crack [Barenblatt 1962; Dugdale 1960; Del Piero and Raous 2010], which automatically contains a critical stress and even a characteristic length. The goal will be to study the influence of the critical stress and characteristic length on the nucleation and the propagation of a crack in the spirit of the previous works based on the variational approach to fracture [Abdelmoula et al. 2010; Bourdin et al. 2008; Charlotte et al. 2006; Del Piero and Truskinovsky 2009; Ferdjani et al. 2007; Giacomini 2005; Jaubert and Marigo 2006; Marigo and Truskinovsky 2004].

\section{References}

[Abdelmoula and Marigo 2000] R. Abdelmoula and J.-J. Marigo, "The effective behavior of a fiber bridged crack", J. Mech. Phys. Solids 48:11 (2000), 2419-2444.

[Abdelmoula et al. 2010] R. Abdelmoula, J.-J. Marigo, and T. Weller, "Construction and justification of Paris-like fatigue laws from Dugdale-type cohesive models", Ann. Solid Struct. Mech. 1:3-4 (2010), 139-158.

[Barenblatt 1962] G. I. Barenblatt, "The mathematical theory of equilibrium cracks in brittle fracture", pp. 55-129 in Advances in Applied Mechanics, vol. 7, Academic Press, New York, 1962.

[Bilteryst and Marigo 2003] F. Bilteryst and J.-J. Marigo, "An energy based analysis of the pull-out problem", Eur. J. Mech. A Solids 22:1 (2003), 55-69. 
[Bonnaillie-Noël et al. 2010] V. Bonnaillie-Noël, M. Dambrine, F. Hérau, and G. Vial, "On generalized Ventcel's type boundary conditions for Laplace operator in a bounded domain", SIAM J. Math. Anal. 42:2 (2010), 931-945.

[Bonnaillie-Noel et al. 2011] V. Bonnaillie-Noel, M. Dambrine, and G. Vial, "Small defects in mechanics", AIP Conference Proceedings 1389:1 (2011), 1416-1419.

[Bourdin et al. 2008] B. Bourdin, G. A. Francfort, and J.-J. Marigo, "The variational approach to fracture", J. Elasticity 91:1-3 (2008), 5-148.

[Bui 1978] H. Bui, Mécanique de la rupture fragile, Masson, 1978.

[Chambolle et al. 2008] A. Chambolle, A. Giacomini, and M. Ponsiglione, "Crack initiation in brittle materials", Arch. Ration. Mech. Anal. 188:2 (2008), 309-349.

[Chambolle et al. 2009] A. Chambolle, G. A. Francfort, and J.-J. Marigo, "When and how do cracks propagate?", J. Mech. Phys. Solids 57:9 (2009), 1614-1622.

[Chambolle et al. 2010] A. Chambolle, G. A. Francfort, and J.-J. Marigo, "Revisiting energy release rates in brittle fracture", J. Nonlinear Sci. 20:4 (2010), 395-424.

[Charlotte et al. 2006] M. Charlotte, J. Laverne, and J.-J. Marigo, "Initiation of cracks with cohesive force models: a variational approach”, Eur. J. Mech. A Solids 25:4 (2006), 649-669.

[Cherepanov 1979] G. Cherepanov, Mechanics of brittle fracture, McGraw-Hill, New York, 1979.

[Dauge 1988] M. Dauge, Elliptic boundary value problems on corner domains: Smoothness and asymptotics of solutions, Lecture Notes in Mathematics 1341, Springer, Berlin, 1988.

[Dauge et al. 2010] M. Dauge, S. Tordeux, and G. Vial, "Selfsimilar perturbation near a corner: matching versus multiscale expansions for a model problem", pp. 95-134 in Around the research of Vladimir Maz'ya, II, edited by A. Laptev, Int. Math. Ser. (N. Y.) 12, Springer, New York, 2010.

[David et al. 2012] M. David, J.-J. Marigo, and C. Pideri, "Homogenized interface model describing inhomogeneities located on a surface”, J. Elasticity 109:2 (2012), 153-187.

[Del Piero and Raous 2010] G. Del Piero and M. Raous, "A unified model for adhesive interfaces with damage, viscosity, and friction”, Eur. J. Mech. A Solids 29:4 (2010), 496-507.

[Del Piero and Truskinovsky 2009] G. Del Piero and L. Truskinovsky, "Elastic bars with cohesive energy", Contin. Mech. Thermodyn. 21:2 (2009), 141-171.

[Destuynder and Djaoua 1981] P. Destuynder and M. Djaoua, "Sur une interprétation mathématique de l'intégrale de Rice en théorie de la rupture fragile", Math. Methods Appl. Sci. 3:1 (1981), 70-87.

[Dugdale 1960] D. Dugdale, "Yielding of steel sheets containing slits", J. Mech. Phys. Solids 8:2 (1960), 100-104.

[Ferdjani et al. 2007] H. Ferdjani, R. Abdelmoula, and J.-J. Marigo, "Insensitivity to small defects of the rupture of materials governed by the Dugdale model", Contin. Mech. Thermodyn. 19:3-4 (2007), 191-210.

[Francfort and Marigo 1998] G. A. Francfort and J.-J. Marigo, "Revisiting brittle fracture as an energy minimization problem”, J. Mech. Phys. Solids 46:8 (1998), 1319-1342.

[Geymonat et al. 2011] G. Geymonat, F. Krasucki, S. Hendili, and M. Vidrascu, "The matched asymptotic expansion for the computation of the effective behavior of an elastic structure with a thin layer of holes", Int. J. Multiscale Comput. Eng. 9:5 (2011), 529-542.

[Giacomini 2005] A. Giacomini, "Size effects on quasi-static growth of cracks", SIAM J. Math. Anal. 36:6 (2005), 1887-1928.

[Griffith 1921] A. A. Griffith, "The phenomena of rupture and flow in solids", Phil. Trans. R. Soc. Lond. A 221 (1921), 163-198. 
[Grisvard 1985] P. Grisvard, Elliptic problems in nonsmooth domains, Monographs and Studies in Mathematics 24, Pitman (Advanced Publishing Program), Boston, 1985.

[Grisvard 1986] P. Grisvard, "Problèmes aux limites dans les polygones: Mode d'emploi", EDF Bull. Direction Études Rech. Sér. C Math. Inform. 1 (1986), 21-59.

[Irwin 1958] G. R. Irwin, "Fracture", pp. 551-590 in Handbuch der Physik, Band 6: Elastizität und Plastizität, edited by S. Flügge, Springer, Berlin, 1958.

[Jaubert and Marigo 2006] A. Jaubert and J.-J. Marigo, "Justification of Paris-type fatigue laws from cohesive forces model via a variational approach", Contin. Mech. Thermodyn. 18:1-2 (2006), 23-45.

[Lawn 1993] B. Lawn, Fracture of brittle solids, 2nd ed., Cambridge University Press, 1993.

[Leblond 2003] J.-B. Leblond, Mécanique de la rupture fragile et ductile, Hermes Science Publications, Paris, 2003.

[Leguillon 1989] D. Leguillon, “Calcul du taux de restitution de l'énergie au voisinage d'une singularité”, C. R. Acad. Sci., Paris, Sér. II 309:10 (1989), 945-950.

[Marigo 2010] J.-J. Marigo, "Initiation of cracks in Griffith's theory: An argument of continuity in favor of global minimization”, J. Nonlinear Sci. 20:6 (2010), 831-868.

[Marigo and Pideri 2011] J.-J. Marigo and C. Pideri, "The effective behavior of elastic bodies containing microcracks or microholes localized on a surface", Int. J. Damage Mech. 20:8 (2011), 11511177.

[Marigo and Truskinovsky 2004] J.-J. Marigo and L. Truskinovsky, "Initiation and propagation of fracture in the models of Griffith and Barenblatt", Contin. Mech. Thermodyn. 16:4 (2004), 391-409.

[Negri 2010] M. Negri, "A comparative analysis on variational models for quasi-static brittle crack propagation”, Adv. Calc. Var. 3:2 (2010), 149-212.

[Negri and Ortner 2008] M. Negri and C. Ortner, "Quasi-static crack propagation by Griffith's criterion”, Math. Models Methods Appl. Sci. 18:11 (2008), 1895-1925.

[Nguyen 2000] Q. S. Nguyen, Stability and nonlinear solid mechanics, Wiley, Chichester, 2000.

[Rice 1968] J. R. Rice, "A path independent integral and the approximate analysis of strain concentration by notches and cracks", J. Appl. Mech. 35:2 (1968), 379-386.

[Vidrascu et al. 2012] M. Vidrascu, G. Geymonat, S. Hendili, and F. Krasucki, "Matched asymptotic expansion and domain decomposition for an elastic structure", pp. 281 in Twenty-first international conference on domain decomposition methods (Rennes, France, June 25-29, 2012), 2012.

Received 18 Dec 2012. Revised 30 Apr 2013. Accepted 5 Jun 2013.

THI BACH TUYET DANG: tuyet@lms.polytechnique.fr

Laboratoire de Mécanique des Solides, École Polytechnique, CNRS, UMR 7649,

91128 Palaiseau cedex, France

LAURENCE HALPERN: halpern@math .univ-paris13.fr

LAGA, Université Paris 13, Sorbonne Paris Cité, CNRS, UMR 7539, 93430 Villetaneuse, France

JEAN-JACQUES MARIGO: marigo@lms .polytechnique.fr

Laboratoire de Mécanique des Solides, École Polytechnique, CNRS, UMR 7649,

91128 Palaiseau cedex, France 



\title{
THE HOMOGENIZED BEHAVIOR OF UNIDIRECTIONAL FIBER-REINFORCED COMPOSITE MATERIALS IN THE CASE OF DEBONDED FIBERS
}

\author{
YAHYA BERREHILI AND JEAN-JACQUES MARIGO
}

\begin{abstract}
This paper is devoted to the analysis of the homogenized behavior of unidirectional composite materials once the fibers are debonded from (but still in contact with) the matrix. This homogenized behavior is built by an asymptotic method in the framework of the homogenization theory. The main result is that the homogenized behavior of the debonded composite is that of a generalized continuous medium with an enriched kinematics. Indeed, besides the usual macroscopic displacement field, the macroscopic kinematics contains two other scalar fields. The former one corresponds to the displacement of the matrix whereas the two latter ones correspond to the sliding and the rotation of the debonded fibers with respect to the matrix. Accordingly, new homogenized coefficients and new coupled equilibrium equations appear. This problem is addressed in a linear elastic three-dimensional setting.
\end{abstract}

\section{Introduction}

The use of unidirectional fiber-reinforced composite materials does not cease to grow in various domains and particularly in the domains of aerospace and aeronautics. This is due to their various properties and especially to their interesting mechanical behavior in terms of their specific effective stiffness in the direction of the fibers. (Throughout the paper, the word effective is a synonym of homogenized or macroscopic.) The effective elastic behavior of such composites is now well known and well modeled by the homogenization theory as long as the fibers are assumed to be perfectly bonded to the matrix [Léné 1984; Michel et al. 1999; Sánchez-Palencia 1980; Suquet 1982].

However, since their mechanical performance is considered optimal when the components remain bonded, it remains to evaluate the loss of performance when

\section{Communicated by Pierre Seppecher.}

This work was partially supported by the French Agence Nationale de la Recherche (ANR), under grant epsilon (BLAN08-2_312370) "Domain decomposition and multi-scale computations of singularities in mechanical structures".

MSC2000: 35C20, 35J20, 74B05, 74G10, 74Q15.

Keywords: homogenization, composite materials, debonding. 
the fibers are debonded. Of course, if one considers that the elastic behavior is due to the matrix alone, the specific stiffness drops drastically. But this type of estimate simply gives a lower bound to the stiffness and one must define more precisely the effective behavior of completely or partially debonded unidirectional composites.

Many works have been devoted to this task; see for instance [Bouchelaghem et al. 2007; Caporale et al. 2006; Gonzàlez and LLorca 2007; Greco 2009; Jendli et al. 2009; Kulkarni et al. 2009; Kushch et al. 2011; Léné and Leguillon 1982; Marigo et al. 1987; Matouš and Geubelle 2006; Moraleda et al. 2009; Teng 2010]. In general, these studies consist in replacing the perfect bond of the interface by some "cohesive law" or simply in removing the fibers when the debonding is complete. In any case, the calculation of the new homogenized mechanical coefficients is performed by considering the usual elementary problems set on the unit cell without reconsidering the general procedure of homogenization. However, when following the two-scale asymptotic approach, it appears that the argument used to obtain that the zero-order displacement field does not depend on the microscopic variable is no longer valid. Therefore, in the zone where the fibers are debonded, the macroscopic displacement field must be replaced by another "macroscopic" displacement field, corresponding to the independent displacement of the fibers [Berrehili and Marigo 2010]. Consequently, one must also construct the macroscopic problem which gives this additional field. That is the purpose of this paper.

Specifically, the paper is organized as follows. The next section is devoted to the setting of the problem: one considers a composite structure $\Omega$, constituted by a periodic distribution of elastic unidirectional fibers whose direction is $\boldsymbol{e}_{3}$ and embedded in an elastic matrix. In a part $\Omega_{c}$ of $\Omega$ the fibers are assumed to be bonded to the matrix whereas in the complementary part $\Omega_{d}$ they are assumed to be debonded but still in contact without friction with the matrix. We then formulate the elastostatic problem which contains the small parameter $\epsilon$ related to the size of the microstructure and which governs the displacement field $\boldsymbol{u}^{\epsilon}$ and the stress field $\boldsymbol{\sigma}^{\epsilon}$. The third section is devoted to the asymptotic analysis, i.e., the behavior of $\boldsymbol{u}^{\epsilon}$ and $\boldsymbol{\sigma}^{\epsilon}$ when $\epsilon$ goes to 0 . Following a two-scale approach, we first postulate that $\boldsymbol{u}^{\epsilon}$ and $\boldsymbol{\sigma}^{\epsilon}$ can be expanded in powers of $\epsilon$, the coefficients $\boldsymbol{u}^{i}(\boldsymbol{x}, \boldsymbol{y})$ and $\boldsymbol{\sigma}^{i}(\boldsymbol{x}, \boldsymbol{y})$ of the expansion being periodic functions of the microscopic coordinates $y$. We then obtain a sequence of variational equations in terms of the $\boldsymbol{u}^{i}$ and the $\boldsymbol{\sigma}^{i}$. These equations are sequentially solved to finally obtain the effective behavior of the composite in its bonded and debonded parts. In the fourth section, we study the properties of the effective model and, in particular, the properties of the effective coefficients provided by the solutions of linear elastic problems posed either on the bonded or on the debonded cell. Then, some examples are treated. We finally conclude giving some perspectives.

The summation convention on repeated indices is used throughout the paper. 
The set of real numbers, the set of $n$-dimensional vectors and the set of symmetric second-order $n$-dimensional tensors are, respectively, denoted by $\mathbb{R}, \mathbb{R}^{n}$ and $\mathbb{M}_{s}^{n}$. Vectors and second-order tensors are indicated by boldface letters, like $\boldsymbol{u}$ and $\boldsymbol{\sigma}$ for the displacement field and the stress field. Their components are denoted by italic letters, like $u_{i}$ and $\sigma_{i j}$. Fourth-order tensors as well as their components are indicated by sans-serif letters, like A or $\mathrm{A}_{i j k l}$ for the stiffness tensor. Such tensors are considered as linear maps acting on second-order tensors. The application of A to $\boldsymbol{\varepsilon}$ is denoted $A \boldsymbol{\varepsilon}$, with components $\mathrm{A}_{i j k l} \varepsilon_{k l}$. The inner product between two vectors or two tensors of the same order is indicated by a dot, like $\boldsymbol{a} \cdot \boldsymbol{b}$ which stands for $a_{i} b_{i}$ or $\boldsymbol{\sigma} \cdot \boldsymbol{\varepsilon}$ for $\sigma_{i j} \varepsilon_{i j}$. The symbol $\otimes$ denotes the tensor product and $\otimes_{s}$ denotes its symmetric part; i.e., $2 \boldsymbol{e}_{1} \otimes_{s} \boldsymbol{e}_{2}=\boldsymbol{e}_{1} \otimes \boldsymbol{e}_{2}+\boldsymbol{e}_{2} \otimes \boldsymbol{e}_{1}$.

In our frequent use of multiple scaling techniques, we adopt the related notation. For instance, $\boldsymbol{x}=\left(x_{1}, x_{2}, x_{3}\right)$ always denotes a macroscopic coordinate while $\boldsymbol{y}=\left(y_{1}, y_{2}\right)$ represents a microscopic one. Since the fibers are oriented along the direction $\boldsymbol{e}_{3}$, we distinguish the longitudinal coordinate $x_{3}$ from the transversal coordinates $\boldsymbol{x}^{\prime}=\left(x_{1}, x_{2}\right)$. Latin indices run from 1 to 3 , while Greek indices run from 1 to 2 . When a spatial (scalar, vectorial or tensorial) field depends both on $\boldsymbol{x}$ and $\boldsymbol{y}$, the partial derivative with respect to one of the coordinates appears explicitly as an index: for example, $\operatorname{div}_{x} \sigma$ and $\varepsilon_{x}(\boldsymbol{v})$ denote, respectively, the divergence of the stress tensor field $\sigma$ and the symmetric gradient of the vector field $v$ with respect to $\boldsymbol{x}$, while $\operatorname{div}_{\boldsymbol{y}} \sigma$ and $\varepsilon_{\boldsymbol{y}}(\boldsymbol{v})$ are the corresponding derivatives with respect to $\boldsymbol{y}$ :

$$
\begin{gathered}
\operatorname{div}_{\boldsymbol{x}} \boldsymbol{\sigma}(\boldsymbol{x}, \boldsymbol{y})=\frac{\partial \sigma_{i j}}{\partial x_{j}}(\boldsymbol{x}, \boldsymbol{y}) \boldsymbol{e}_{i}, \quad \operatorname{div}_{\boldsymbol{y}} \boldsymbol{\sigma}(\boldsymbol{x}, \boldsymbol{y})=\frac{\partial \sigma_{i \beta}}{\partial y_{\beta}}(\boldsymbol{x}, \boldsymbol{y}) \boldsymbol{e}_{i}, \\
\boldsymbol{\varepsilon}_{\boldsymbol{x}}(\boldsymbol{v})(\boldsymbol{x}, \boldsymbol{y})=\left(\frac{\partial v_{j}}{\partial x_{i}}(\boldsymbol{x}, \boldsymbol{y})+\frac{\partial v_{i}}{\partial x_{j}}(\boldsymbol{x}, \boldsymbol{y})\right) \boldsymbol{e}_{i} \otimes_{s} \boldsymbol{e}_{j}, \\
\boldsymbol{\varepsilon}_{\boldsymbol{y}}(\boldsymbol{v})(\boldsymbol{x}, \boldsymbol{y})=\left(\frac{\partial v_{\alpha}}{\partial y_{\beta}}(\boldsymbol{x}, \boldsymbol{y})+\frac{\partial v_{\beta}}{\partial y_{\alpha}}(\boldsymbol{x}, \boldsymbol{y})\right) \boldsymbol{e}_{\alpha} \otimes_{s} \boldsymbol{e}_{\beta}+\frac{\partial v_{3}}{\partial y_{\alpha}}(\boldsymbol{x}, \boldsymbol{y}) \boldsymbol{e}_{\alpha} \otimes_{s} \boldsymbol{e}_{3} .
\end{gathered}
$$

On a surface $\boldsymbol{I}$ across which a field $f$ is discontinuous, we denote by $\llbracket f \rrbracket$ its jump discontinuity.

\section{Statement of the problem}

We consider a heterogeneous elastic body whose natural reference configuration is a bounded open domain $\Omega$ of $\mathbb{R}^{3}$ with a smooth boundary $\partial \Omega$. We denote by $\left(\boldsymbol{e}_{1}, \boldsymbol{e}_{2}, \boldsymbol{e}_{3}\right)$ the canonical basis of $\mathbb{R}^{3}$ and by $\left(x_{1}, x_{2}, x_{3}\right)$ the coordinates of a point $\boldsymbol{x} \in \Omega$. The body is made of two isotropic linearly elastic materials, called the fibers and the matrix, whose Lamé coefficients and mass density are, respectively, $\left(\lambda_{f}, \mu_{f}, \rho_{f}\right)$ and $\left(\lambda_{m}, \mu_{m}, \rho_{m}\right)$. The fibers are aligned in the direction $\boldsymbol{e}_{3}$ and have a circular cross-section with radius $\epsilon R$. They are periodically distributed in the 


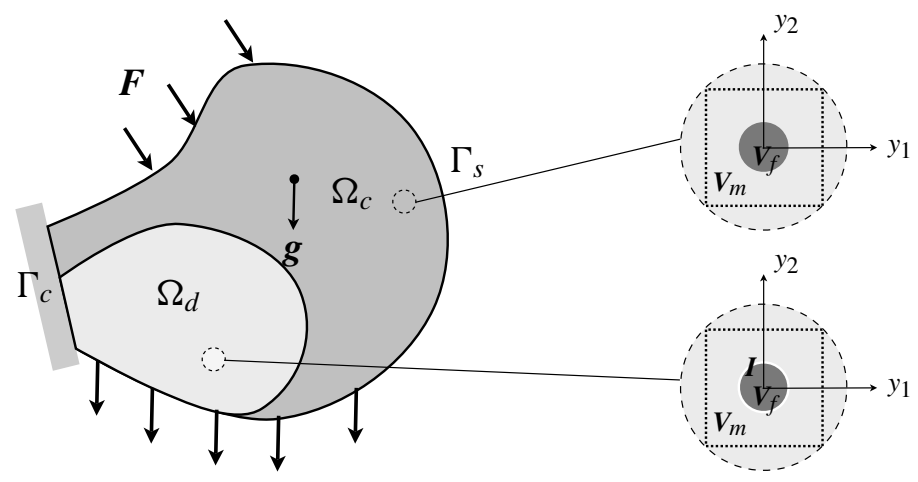

Figure 1. The composite structure and the two periodic cells.

matrix, $\epsilon \boldsymbol{a}$ and $\epsilon \boldsymbol{b}$ being the two vectors of the plane $\left(\boldsymbol{e}_{1}, \boldsymbol{e}_{2}\right)$ characterizing the periodicity. The number of fibers is large so that the dimensionless parameter $\epsilon$ characterizing the fineness of the microstructure (for instance, the ratio between the spatial period and the size of the structure) is small. The domain occupied by the fibers is $\Omega_{f}^{\epsilon}$, that occupied by the matrix is $\Omega_{m}^{\epsilon}$, while the set of all interfaces between fibers and matrix is $\boldsymbol{I}^{\epsilon}$. Accordingly, one has

$$
\Omega=\Omega_{f}^{\epsilon} \cup \boldsymbol{I}^{\epsilon} \cup \Omega_{m}^{\epsilon} .
$$

The fibers are perfectly bonded in a part $\Omega_{c}$ of $\Omega$ and debonded in the complementary part $\Omega_{d}$; see Figure 1 . Both parts contain a large number of fibers and will be considered as given and independent of $\epsilon$. Moreover we assume that in $\Omega_{d}$ the fibers remain in contact with the matrix but can slip without friction. Accordingly, denoting by

$$
\boldsymbol{I}_{c}^{\epsilon}=\Omega_{c} \cap \boldsymbol{I}^{\epsilon}, \quad \boldsymbol{I}_{d}^{\epsilon}=\Omega_{d} \cap \boldsymbol{I}^{\epsilon},
$$

respectively, the bonded and debonded interfaces, the interface conditions in terms of the displacement and the stress fields read as

$$
\left\{\begin{array}{cc}
\llbracket u \rrbracket=0, \quad \llbracket \sigma \rrbracket n=0 & \text { on } \boldsymbol{I}_{c}^{\epsilon}, \\
\llbracket u \rrbracket \cdot n=0, \quad \llbracket \sigma \rrbracket n \cdot n=0, \quad \boldsymbol{\sigma} n \wedge \boldsymbol{n}=0 & \text { on } \boldsymbol{I}_{d}^{\epsilon} .
\end{array}\right.
$$

In (6), $\boldsymbol{n}$ is the outer normal to the fiber at an interface and the brackets denote the jump of the involved field across the interface. The conditions on $\boldsymbol{I}_{c}^{\epsilon}$ mean that the displacement and the vector stress are continuous; the conditions on $\boldsymbol{I}_{d}^{\epsilon}$ mean that the normal displacement and the normal stress are continuous while the shear stress vanishes.

Remark 1. In the above conditions on the interface between the fibers and the matrix after debonding, we assume that contact always occurs without friction. This allows us to treat linear elastic problems and then the analysis is simplified. 
It would be easy to follow the same procedure by assuming that the fibers are no longer in contact with the matrix after debonding. It is more difficult to consider unilateral frictionless contact conditions where the contact conditions depend on the sign of the normal stress. That leads to nonlinear (but still elastic) problems where the superposition principle can no longer be used. Much more difficult is the case where the contact occurs with friction. Then the effective behavior is no longer elastic and one must introduce internal variables. All these more elaborated cases are outside the scope of this didactic paper and will be the subject of future works.

The body is submitted to a specific body force density $\boldsymbol{g}$ (independent of $\epsilon$ ). The part $\Gamma_{c}$ of the boundary $\partial \Omega$ is fixed while the complementary part $\Gamma_{s}=\partial \Omega \backslash \Gamma_{c}$ is submitted to a surface force density $\boldsymbol{F}$ (independent of $\epsilon$ ).

We are now in a position to set the problem which governs the response of the body at equilibrium under the given loading. For a fixed $\epsilon>0$, the problem consists in finding a displacement field $u^{\epsilon}$ and a stress field $\sigma^{\epsilon}$, such that:

Equilibrium: $\begin{cases}\operatorname{div} \sigma^{\epsilon}+\rho_{f} g=0 & \text { in } \Omega_{f}^{\epsilon}, \\ \operatorname{div} \sigma^{\epsilon}+\rho_{m} g=0 & \text { in } \Omega_{m}^{\epsilon},\end{cases}$

Constitutive relations: $\quad \begin{cases}\sigma^{\epsilon}=\lambda_{f} \operatorname{div} \boldsymbol{u}^{\epsilon} \delta+2 \mu_{f} \boldsymbol{\varepsilon}\left(\boldsymbol{u}^{\epsilon}\right) & \text { in } \Omega_{f}^{\epsilon}, \\ \sigma^{\epsilon}=\lambda_{m} \operatorname{div} \boldsymbol{u}^{\epsilon} \boldsymbol{\delta}+2 \mu_{m} \boldsymbol{\varepsilon}\left(\boldsymbol{u}^{\epsilon}\right) & \text { in } \Omega_{m}^{\epsilon},\end{cases}$

Compatibility: $\quad 2 \boldsymbol{\varepsilon}\left(\boldsymbol{u}^{\epsilon}\right)=\nabla \boldsymbol{u}^{\epsilon}+\nabla^{T} \boldsymbol{u}^{\epsilon} \quad$ in $\Omega_{f}^{\epsilon} \cup \Omega_{m}^{\epsilon}$,

Boundary conditions: $\left\{\begin{aligned} \boldsymbol{u}^{\epsilon} & =\mathbf{0} & & \text { on } \Gamma_{c}, \\ \boldsymbol{\sigma}^{\epsilon} \boldsymbol{n} & =\boldsymbol{F} & & \text { on } \Gamma_{s},\end{aligned}\right.$

Interface conditions: $\left\{\begin{array}{cc}\llbracket \boldsymbol{u}^{\epsilon} \rrbracket=\mathbf{0}, \quad \llbracket \boldsymbol{\sigma}^{\epsilon} \rrbracket \boldsymbol{n}=\mathbf{0} & \text { on } \boldsymbol{I}_{c}^{\epsilon}, \\ \llbracket u_{n}^{\epsilon} \rrbracket=0, \quad \boldsymbol{\sigma}^{\epsilon} \boldsymbol{n}=\sigma_{n n}^{\epsilon} \boldsymbol{n}, \quad \llbracket \sigma_{n n}^{\epsilon} \rrbracket=0 & \text { on } \boldsymbol{I}_{d}^{\epsilon} .\end{array}\right.$

In (8), $\delta$ is the identity tensor with $\delta_{i j}=1$ when $i=j$ and $\delta_{i j}=0$ when $i \neq j$. This set of equations constitutes a linear boundary value problem which can be written in a variational form as follows.

Let $\mathscr{C}^{\epsilon}$ be the linear space of kinematically admissible displacement fields; i.e.,

$$
\mathscr{C}^{\epsilon}=\left\{\boldsymbol{v} \in H^{1}\left(\Omega \backslash \boldsymbol{I}_{d}^{\epsilon} ; \mathbb{R}^{3}\right): \llbracket \boldsymbol{v} \rrbracket \cdot \boldsymbol{n}=\mathbf{0} \text { on } \boldsymbol{I}_{d}^{\epsilon}, \boldsymbol{v}=\mathbf{0} \text { on } \Gamma_{c}\right\},
$$

let $\mathrm{f}^{\epsilon}$ be the continuous linear form associated with the applied forces; i.e.,

$$
f^{\epsilon}(v)=\int_{\Omega_{f}^{\epsilon}} \rho_{f} \boldsymbol{g} \cdot \boldsymbol{v} d x+\int_{\Omega_{m}^{\epsilon}} \rho_{m} \boldsymbol{g} \cdot \boldsymbol{v} d x+\int_{\Gamma_{s}} \boldsymbol{F} \cdot \boldsymbol{v} d \Gamma \quad \text { for } \boldsymbol{v} \in \mathscr{C}^{\epsilon},
$$

and let $a^{\epsilon}$ be the bilinear continuous form associated with the elastic energy; i.e.,

$$
\mathrm{a}^{\epsilon}(\boldsymbol{u}, \boldsymbol{v})=\int_{\Omega_{f}^{\epsilon}} \mathrm{A}^{f} \boldsymbol{\varepsilon}(\boldsymbol{u}) \cdot \boldsymbol{\varepsilon}(\boldsymbol{v}) d x+\int_{\Omega_{m}^{\epsilon}} \mathrm{A}^{m} \boldsymbol{\varepsilon}(\boldsymbol{u}) \cdot \boldsymbol{\varepsilon}(\boldsymbol{v}) d x .
$$


In (14), $\mathrm{A}^{f}$ and $\mathrm{A}^{m}$ stand for the fourth-order elasticity tensors of the fibers and the matrix, respectively; i.e.,

$$
\mathrm{A}_{i j k l}^{f, m}=\lambda_{f, m} \delta_{i j} \delta_{k l}+\mu_{f, m}\left(\delta_{i k} \delta_{j l}+\delta_{i l} \delta_{j k}\right) .
$$

Then $\boldsymbol{u}^{\epsilon}$ must satisfy the variational problem

$$
\text { find } \boldsymbol{u}^{\epsilon} \in \mathscr{C}^{\epsilon} \quad \text { such that } a^{\epsilon}\left(\boldsymbol{u}^{\epsilon}, \boldsymbol{v}\right)=\mathrm{f}^{\epsilon}(\boldsymbol{v}) \text { for all } \boldsymbol{v} \in \mathscr{C}^{\epsilon},
$$

and $\sigma^{\epsilon}$ is the associated stress field given in terms of the strain field by (8). The existence and the uniqueness of the solution $\boldsymbol{u}^{\epsilon}$ of (16) is guaranteed provided that the boundary $\Gamma_{c}$ is such that there does not exist any (nonzero) rigid displacement which is kinematically admissible. Specifically, let us denote by $\mathscr{R}^{\epsilon}$ the set of displacement fields which are both kinematically admissible and corresponding to a null strain field; i.e.,

$$
\mathscr{R}^{\epsilon}=\left\{\boldsymbol{v} \in \mathscr{C}^{\epsilon}: \boldsymbol{\varepsilon}(\boldsymbol{v})=\mathbf{0} \text { in } \Omega \backslash \boldsymbol{I}_{d}^{\epsilon}\right\} .
$$

By standard arguments, we have:

Proposition 1. Under the condition that $\mathscr{R}^{\epsilon}=\{\mathbf{0}\}$ and that the density of forces $\boldsymbol{g}$ and $\boldsymbol{F}$ are smooth enough, the variational problem (16) admits a unique solution $\boldsymbol{u}^{\epsilon}$.

The necessary and sufficient condition above for the existence and the uniqueness of the solution depends in general both on $\Gamma_{c}$ and $\Omega_{d}$. However, the existence of a solution is guaranteed if $\mathscr{R}^{\epsilon}=\{\mathbf{0}\}$, that is, if no rigid displacements are allowed. We will assume henceforth that this condition is satisfied.

\section{Asymptotic analysis}

This section is devoted to the behavior of $\boldsymbol{u}^{\epsilon}$, the unique solution of (16), when $\epsilon$ goes to 0 . For that we use a formal double-scale asymptotic method like in [Abdelmoula and Marigo 2000; Allaire 1992; Bensoussan et al. 1978; David et al. 2012; Marigo and Pideri 2011]. The goal is not to obtain rigorous results of convergence, but simply to formally construct the "limit" problem.

3.1. The assumed asymptotic expansion of $\boldsymbol{u}^{\epsilon}$. By virtue of the unidirectional character of the fibers, one can choose a two-dimensional domain $\boldsymbol{V}$ as the rescaled periodic cell characterizing the spatial distribution of the fibers; see [Bouchelaghem et al. 2007; Léné 1984; Marigo and Pideri 2011]. The fiber part and the matrix part of this cell are, respectively, the open sets $\boldsymbol{V}_{f}$ and $\boldsymbol{V}_{m}$ of the $\left(y_{1}, y_{2}\right)$ plane, while the interface is $\boldsymbol{I}=\partial \boldsymbol{V}_{f} \cap \partial \boldsymbol{V}_{m}$. Accordingly, one has

$$
\boldsymbol{V}=\boldsymbol{V}_{f} \cup \boldsymbol{I} \cup \boldsymbol{V}_{m}
$$


Moreover, the rigidity tensor and the mass density fields can be read as

$$
\begin{aligned}
& \mathrm{A}^{\epsilon}(\boldsymbol{x})=\mathrm{A}\left(\frac{\boldsymbol{x}^{\prime}}{\epsilon}\right) \quad \text { with } \mathrm{A}(\boldsymbol{y})= \begin{cases}\mathrm{A}^{f} & \text { if } \boldsymbol{y} \in \boldsymbol{V}_{f}, \\
\mathrm{~A}^{m} & \text { if } \boldsymbol{y} \in \boldsymbol{V}_{m},\end{cases} \\
& \rho^{\epsilon}(\boldsymbol{x})=\rho\left(\frac{\boldsymbol{x}^{\prime}}{\epsilon}\right) \quad \text { with } \rho(\boldsymbol{y})= \begin{cases}\rho_{f} & \text { if } \boldsymbol{y} \in \boldsymbol{V}_{f}, \\
\rho_{m} & \text { if } \boldsymbol{y} \in \boldsymbol{V}_{m} .\end{cases}
\end{aligned}
$$

This allows us to write problem (16) in the equivalent form

find $\boldsymbol{u}^{\epsilon} \in \mathscr{C}^{\epsilon} \quad$ such that $\int_{\Omega \backslash \boldsymbol{I}_{d}^{\epsilon}} \mathrm{A}^{\epsilon} \boldsymbol{\varepsilon}\left(\boldsymbol{u}^{\epsilon}\right) \cdot \boldsymbol{\varepsilon}(\boldsymbol{v}) d x=\int_{\Omega} \rho^{\epsilon} \boldsymbol{g} \cdot \boldsymbol{v} d x+\int_{\Gamma_{s}} \boldsymbol{F} \cdot \boldsymbol{v} d \Gamma$

$$
\text { for all } v \in \mathscr{C}^{\epsilon} \text {. }
$$

Following the classical two-scale procedure in homogenization theory of periodic media [Allaire 1992; Bensoussan et al. 1978], we assume that $\boldsymbol{u}^{\epsilon}$ can be expanded as follows:

$$
\boldsymbol{u}^{\epsilon}(\boldsymbol{x})=\sum_{i=0}^{\infty} \epsilon^{i} \boldsymbol{u}^{i}\left(\boldsymbol{x}, \frac{\boldsymbol{x}^{\prime}}{\epsilon}\right),
$$

where the fields $\boldsymbol{u}^{i}$ are defined in $\Omega \times V$ and $\boldsymbol{V}$-periodic (with respect to the microscopic variable $\boldsymbol{y}$ ). As far as their regularity with respect to $\boldsymbol{y}$ is concerned, one can discriminate according to whether $\boldsymbol{x}$ belongs to $\Omega_{c}$ or $\Omega_{d}$. Specifically, if $\boldsymbol{x} \in \Omega_{c}$, then $\boldsymbol{u}^{i}(\boldsymbol{x}, \cdot)$ must be continuous across $\boldsymbol{I}$, while if $\boldsymbol{x} \in \Omega_{d}$, then $u_{n}^{i}(\boldsymbol{x}, \cdot)$ only must be continuous across $\boldsymbol{I}$.

Using the chain rule, the strain field admits the expansion

$$
\boldsymbol{\varepsilon}\left(\boldsymbol{u}^{\epsilon}\right)(\boldsymbol{x})=\sum_{i=-1}^{\infty} \epsilon^{i}\left(\boldsymbol{\varepsilon}_{\boldsymbol{y}}\left(\boldsymbol{u}^{i+1}\right)\left(\boldsymbol{x}, \frac{\boldsymbol{x}^{\prime}}{\epsilon}\right)+\boldsymbol{\varepsilon}_{\boldsymbol{x}}\left(\boldsymbol{u}^{i}\right)\left(\boldsymbol{x}, \frac{\boldsymbol{x}^{\prime}}{\epsilon}\right)\right),
$$

where $\boldsymbol{\varepsilon}_{\boldsymbol{x}}(\boldsymbol{v})$ and $\boldsymbol{\varepsilon}_{\boldsymbol{y}}(\boldsymbol{v})$ denote, respectively, the symmetrized gradient of the displacement field $\boldsymbol{v}$ with respect to the macroscopic and microscopic coordinates; see (2)-(3).

3.2. Equations at various orders. Let us choose a two-scale smooth displacement field $\boldsymbol{v}^{\epsilon}(\boldsymbol{x})=\boldsymbol{v}\left(\boldsymbol{x}, \boldsymbol{x}^{\prime} / \epsilon\right), \boldsymbol{V}$-periodic and such that $\boldsymbol{v}(\boldsymbol{x}, \boldsymbol{y})=\mathbf{0}$ when $\boldsymbol{x} \in \Gamma_{c}$, as an element of $\mathscr{C}^{\epsilon}$ and let us insert it into (21) as the test field. After inserting the asymptotic expansion of $\boldsymbol{u}^{\epsilon}$ into (21) and identifying the terms at the same power of $\epsilon$, one obtains a sequence of variational problems for the $\boldsymbol{u}^{i}$, the first three of which are given below. (One formally replaces simple integrals over $\Omega$ by multiple integrals over $\Omega \times \boldsymbol{V}$ in the spirit of the double-scale approach [Allaire 1992].)

(1) At order $\epsilon^{-2}$ :

$$
0=\int_{\Omega_{c}} \int_{V} \mathrm{~A} \boldsymbol{\varepsilon}_{\boldsymbol{y}}\left(\boldsymbol{u}^{0}\right) \cdot \boldsymbol{\varepsilon}_{\boldsymbol{y}}(\boldsymbol{v}) d y d x+\int_{\Omega_{d}} \int_{\boldsymbol{V} \backslash \boldsymbol{I}} \mathrm{A} \boldsymbol{\varepsilon}_{\boldsymbol{y}}\left(\boldsymbol{u}^{0}\right) \cdot \boldsymbol{\varepsilon}_{\boldsymbol{y}}(\boldsymbol{v}) d y d x .
$$


(2) At order $\epsilon^{-1}$ :

$$
\begin{array}{r}
0=\int_{\Omega_{c}} \int_{V} \mathrm{~A} \boldsymbol{\varepsilon}_{\boldsymbol{y}}\left(\boldsymbol{u}^{0}\right) \cdot \boldsymbol{\varepsilon}_{\boldsymbol{x}}(\boldsymbol{v}) d y d x+\int_{\Omega_{d}} \int_{\boldsymbol{V} \backslash \boldsymbol{I}} \mathrm{A} \boldsymbol{\varepsilon}_{\boldsymbol{y}}\left(\boldsymbol{u}^{0}\right) \cdot \boldsymbol{\varepsilon}_{\boldsymbol{x}}(\boldsymbol{v}) d y d x \\
\quad+\int_{\Omega_{c}} \int_{V} \mathrm{~A}\left(\boldsymbol{\varepsilon}_{\boldsymbol{y}}\left(\boldsymbol{u}^{1}\right)+\boldsymbol{\varepsilon}_{\boldsymbol{x}}\left(\boldsymbol{u}^{0}\right)\right) \cdot \boldsymbol{\varepsilon}_{\boldsymbol{y}}(\boldsymbol{v}) d y d x \\
\quad+\int_{\Omega_{d}} \int_{\boldsymbol{V} \backslash \boldsymbol{I}} \mathrm{A}\left(\boldsymbol{\varepsilon}_{\boldsymbol{y}}\left(\boldsymbol{u}^{1}\right)+\boldsymbol{\varepsilon}_{\boldsymbol{x}}\left(\boldsymbol{u}^{0}\right)\right) \cdot \boldsymbol{\varepsilon}_{\boldsymbol{y}}(\boldsymbol{v}) d y d x
\end{array}
$$

(3) At order $\epsilon^{0}$ :

$$
\begin{array}{r}
\int_{\Omega_{c}} \int_{V} \mathrm{~A}\left(\boldsymbol{\varepsilon}_{\boldsymbol{y}}\left(\boldsymbol{u}^{2}\right)+\boldsymbol{\varepsilon}_{\boldsymbol{x}}\left(\boldsymbol{u}^{1}\right)\right) \cdot \boldsymbol{\varepsilon}_{\boldsymbol{y}}(\boldsymbol{v}) d y d x+\int_{\Omega_{d}} \int_{\boldsymbol{V} \backslash \boldsymbol{I}} \mathrm{A}\left(\boldsymbol{\varepsilon}_{\boldsymbol{y}}\left(\boldsymbol{u}^{2}\right)+\boldsymbol{\varepsilon}_{\boldsymbol{x}}\left(\boldsymbol{u}^{1}\right)\right) \cdot \boldsymbol{\varepsilon}_{\boldsymbol{y}}(\boldsymbol{v}) d y d x \\
+\int_{\Omega_{c}} \int_{V} \mathrm{~A}\left(\boldsymbol{\varepsilon}_{\boldsymbol{y}}\left(\boldsymbol{u}^{1}\right)+\boldsymbol{\varepsilon}_{\boldsymbol{x}}\left(\boldsymbol{u}^{0}\right)\right) \cdot \boldsymbol{\varepsilon}_{\boldsymbol{x}}(\boldsymbol{v}) d y d x+\int_{\Omega_{d}} \int_{V \backslash \boldsymbol{I}} \mathrm{A}\left(\boldsymbol{\varepsilon}_{\boldsymbol{y}}\left(\boldsymbol{u}^{1}\right)+\boldsymbol{\varepsilon}_{\boldsymbol{x}}\left(\boldsymbol{u}^{0}\right)\right) \cdot \boldsymbol{\varepsilon}_{\boldsymbol{x}}(\boldsymbol{v}) d y d x \\
=\int_{\Omega} \int_{V} \rho \boldsymbol{g} \cdot \boldsymbol{v} d y d x+\int_{\Gamma_{s}} \int_{V} \boldsymbol{F} \cdot \boldsymbol{v} d y d \Gamma .
\end{array}
$$

In (24)-(26), A and $\rho$ stand for the $\boldsymbol{V}$-periodic functions of $\boldsymbol{y}$ introduced in (19) and (20). Moreover, these variational equalities must hold for any smooth $\boldsymbol{v}(\boldsymbol{x}, \boldsymbol{y})$ which vanishes when $\boldsymbol{x} \in \Gamma_{c}$ as a function of $\boldsymbol{x}$, which is $\boldsymbol{V}$-periodic in $\boldsymbol{y}$, continuous across $\boldsymbol{I}$ when $\boldsymbol{x} \in \Omega_{c}$ and whose normal component $v_{n}$ is continuous across $\boldsymbol{I}$ when $\boldsymbol{x} \in \Omega_{d}$.

3.3. The form of $\boldsymbol{u}^{\mathbf{0}}$. By choosing $\boldsymbol{v}=\boldsymbol{u}^{0}$ in (24) (which is licit) and owing to the positivity of the elasticity tensors $\mathrm{A}^{f}$ and $\mathrm{A}^{m}$, one deduces that

$$
\boldsymbol{\varepsilon}_{\boldsymbol{y}}\left(\boldsymbol{u}^{0}\right)=\mathbf{0} \quad \text { in } \Omega_{c} \times \boldsymbol{V} \text { and in } \Omega_{d} \times(\boldsymbol{V} \backslash \boldsymbol{I}) .
$$

Let us discriminate the case when $x \in \Omega_{c}$ and that when $x \in \Omega_{d}$.

(1) When $\boldsymbol{x} \in \Omega_{c}$, since $\boldsymbol{\varepsilon}\left(\boldsymbol{u}^{0}\right)(\boldsymbol{x}, \boldsymbol{y})=\mathbf{0}$ for all $\boldsymbol{y} \in V, \boldsymbol{u}^{0}$ must be a rigid displacement with respect to $\boldsymbol{y}$. Recalling that $\boldsymbol{u}^{0}(\boldsymbol{x}, \boldsymbol{y}) \in \mathbb{R}^{3}$ and that $\boldsymbol{y}=\left(y_{1}, y_{2}\right)$, using (3) leads to

$$
\boldsymbol{u}^{0}(\boldsymbol{x}, \boldsymbol{y})=\boldsymbol{u}(\boldsymbol{x})+\omega(\boldsymbol{x}) \boldsymbol{e}_{3} \wedge \boldsymbol{y} \text { for all } \boldsymbol{y} \in \boldsymbol{V},
$$

where $\boldsymbol{u}(\boldsymbol{x}) \in \mathbb{R}^{3}$ and $\omega(\boldsymbol{x}) \in \mathbb{R}$. (Note that the rotations of axes $\boldsymbol{e}_{1}$ and $\boldsymbol{e}_{2}$ are automatically eliminated because $\boldsymbol{u}^{0}$ is independent of $y_{3}$.) But since $\boldsymbol{u}^{0}$ must be $\boldsymbol{V}$-periodic, one gets also $\omega(\boldsymbol{x})=0$. Finally, we have obtained that

$$
\text { for } \boldsymbol{x} \in \Omega_{c}: \quad \boldsymbol{u}^{0}(\boldsymbol{x}, \boldsymbol{y})=\boldsymbol{u}(\boldsymbol{x}) \quad \text { for all } \boldsymbol{y} \in \boldsymbol{V} .
$$

This result is the classical property of the homogenization theory which states that the leading term of the asymptotic displacement field expansion does not depend on the microscopic coordinates. However, this property holds true only because the fiber is perfectly bonded to the matrix, as we will see hereafter. 
(2) When $\boldsymbol{x} \in \Omega_{d}$, one has separately $\boldsymbol{\varepsilon}_{\boldsymbol{y}}\left(\boldsymbol{u}^{0}\right)(\boldsymbol{x}, \cdot)=\mathbf{0}$ in $\boldsymbol{V}_{f}$ and in $\boldsymbol{V}_{m}$. Therefore, $\boldsymbol{u}^{0}(\boldsymbol{x}, \boldsymbol{y})$ must be a rigid displacement field with respect to $\boldsymbol{y}$ in the matrix part $\boldsymbol{V}_{m}$ and a priori another rigid displacement field in the fiber part $\boldsymbol{V}_{f}$ of the cell $\boldsymbol{V}$. Accordingly, $\boldsymbol{u}^{0}(\boldsymbol{x}, \boldsymbol{y})$ must read as

$$
\boldsymbol{u}^{0}(\boldsymbol{x}, \boldsymbol{y})= \begin{cases}\boldsymbol{u}_{m}(\boldsymbol{x})+\omega_{m}(\boldsymbol{x}) \boldsymbol{e}_{3} \wedge \boldsymbol{y} & \text { for all } \boldsymbol{y} \in \boldsymbol{V}_{m}, \\ \boldsymbol{u}_{f}(\boldsymbol{x})+\omega_{f}(\boldsymbol{x}) \boldsymbol{e}_{3} \wedge \boldsymbol{y} & \text { for all } \boldsymbol{y} \in \boldsymbol{V}_{f},\end{cases}
$$

where $\boldsymbol{u}_{m}(\boldsymbol{x})$ and $\boldsymbol{u}_{f}(\boldsymbol{x})$ are in $\mathbb{R}^{3}, \omega_{m}(\boldsymbol{x})$ and $\omega_{f}(\boldsymbol{x})$ are in $\mathbb{R}$. Since $\boldsymbol{u}^{0}$ must be $\boldsymbol{V}$-periodic, one still gets $\omega_{m}(\boldsymbol{x})=0$. Let us write now the continuity of $u_{n}^{0}$ across $\boldsymbol{I}$. We can take the center of the (circular) fiber cross-section as the origin of the $\left(y_{1}, y_{2}\right)$ plane without loss of generality. Accordingly, $\boldsymbol{n}=\boldsymbol{y} / R=\cos \theta \boldsymbol{e}_{1}+\sin \theta \boldsymbol{e}_{2}$ for $\boldsymbol{y} \in \boldsymbol{I}$. Therefore, $\llbracket \boldsymbol{u}^{0} \rrbracket \cdot \boldsymbol{n}=0$ on $\boldsymbol{I}$ reads as

$$
\cos \theta\left(\boldsymbol{u}_{m}(\boldsymbol{x})-\boldsymbol{u}_{f}(\boldsymbol{x})\right) \cdot \boldsymbol{e}_{1}+\sin \theta\left(\boldsymbol{u}_{m}(\boldsymbol{x})-\boldsymbol{u}_{f}(\boldsymbol{x})\right) \cdot \boldsymbol{e}_{2}=0 \quad \text { for all } \theta \in[0,2 \pi],
$$

from which one immediately deduces that $\boldsymbol{u}_{f}(\boldsymbol{x})=\boldsymbol{u}_{m}(\boldsymbol{x})+\delta(\boldsymbol{x}) \boldsymbol{e}_{3}$. Finally, we have obtained that

$$
\text { for } \boldsymbol{x} \in \Omega_{d}: \quad \boldsymbol{u}^{0}(\boldsymbol{x}, \boldsymbol{y})=\left\{\begin{array}{cl}
\boldsymbol{u}(\boldsymbol{x}) & \text { for all } \boldsymbol{y} \in \boldsymbol{V}_{m}, \\
\boldsymbol{u}(\boldsymbol{x})+\delta(\boldsymbol{x}) \boldsymbol{e}_{3}+\omega(\boldsymbol{x}) \boldsymbol{e}_{3} \wedge \boldsymbol{y} & \text { for all } \boldsymbol{y} \in \boldsymbol{V}_{f} .
\end{array}\right.
$$

For future reference, let us denote by $\mathscr{R}_{d}$ the set of the $V$-periodic displacement fields $\boldsymbol{w}$ such that $\boldsymbol{\varepsilon}_{\boldsymbol{y}}(\boldsymbol{w})=0$ in $\boldsymbol{V} \backslash \boldsymbol{I}$ and $\llbracket w_{n} \rrbracket=0$ on $\boldsymbol{I}$; i.e.,

$$
\mathscr{R}_{d}=\left\{\boldsymbol{w}: \boldsymbol{w}(\boldsymbol{y})=\left\{\begin{array}{cl}
\boldsymbol{a} & \text { for } \boldsymbol{y} \in \boldsymbol{V}_{m}, \\
\boldsymbol{a}+\delta \boldsymbol{e}_{3}+\omega \boldsymbol{e}_{3} \wedge \boldsymbol{y} & \text { for } \boldsymbol{y} \in \boldsymbol{V}_{f},
\end{array} \boldsymbol{a} \in \mathbb{R}^{3}, \delta \in \mathbb{R}, \omega \in \mathbb{R}\right\} .\right.
$$

Thus $\boldsymbol{u}^{0}(\boldsymbol{x}, \cdot) \in \mathscr{R}_{d}$ when $\boldsymbol{x} \in \Omega_{d}$. This result differs from the usual property of the homogenization theory. Indeed, because of the debonding of the fiber from the matrix, the leading term of the asymptotic displacement field expansion depends here on the microscopic coordinates. Moreover, two new macroscopic scalar fields appear in the effective kinematics of the composite. Specifically, the vector field $\boldsymbol{u}$ represents the macroscopic displacement of the matrix while the scalar fields $\delta$ and $\omega$ represent the longitudinal sliding and the relative rotation of the fibers with respect to the matrix. We have obtained a generalized continuous medium.

Let us summarize all results obtained in this subsection:

Proposition 2. The first-order displacement $\boldsymbol{u}^{0}(\boldsymbol{x}, \boldsymbol{y})$ takes two different forms according to whether $\boldsymbol{x}$ is in $\Omega_{c}$ or in $\Omega_{d}$. Specifically,

$$
\begin{aligned}
& \text { for } \boldsymbol{x} \in \Omega_{c}: \quad \boldsymbol{u}^{0}(\boldsymbol{x}, \boldsymbol{y})=\boldsymbol{u}(\boldsymbol{x}) \text { for all } \boldsymbol{y} \in \boldsymbol{V}, \\
& \text { for } \boldsymbol{x} \in \Omega_{d}: \quad \boldsymbol{u}^{0}(\boldsymbol{x}, \boldsymbol{y})=\left\{\begin{array}{cl}
\boldsymbol{u}(\boldsymbol{x}) & \text { for all } \boldsymbol{y} \in \boldsymbol{V}_{m}, \\
\boldsymbol{u}(\boldsymbol{x})+\delta(\boldsymbol{x}) \boldsymbol{e}_{3}+\omega(\boldsymbol{x}) \boldsymbol{e}_{3} \wedge \boldsymbol{y} & \text { for all } \boldsymbol{y} \in \boldsymbol{V}_{f} .
\end{array}\right.
\end{aligned}
$$

Therefore, the effective kinematic behavior in the debonded part of the composite 
body is that of a generalized continuous medium where appear the sliding and the rotation of the fibers with respect to the matrix.

Remark 2. The macroscopic displacement fields $\boldsymbol{u}, \delta$ and $\omega$ can be defined in the whole domain $\Omega$ but $\delta$ and $\omega$ must vanish in $\Omega_{c}$. Moreover, those fields have to be sufficiently smooth in order that the effective elastic energy be finite. Their smoothness will be specified once the effective behavior is obtained. In the same way, the boundary conditions that $\boldsymbol{u}, \delta$ and $\omega$ have to satisfy on $\Gamma_{c}$ will be specified later.

3.4. The elementary cell problems. Inserting (27) and (28) into (25) leads to

$$
\begin{aligned}
0=\int_{\Omega_{c}} & \int_{V} \mathrm{~A}\left(\boldsymbol{\varepsilon}_{\boldsymbol{y}}\left(\boldsymbol{u}^{1}\right)+\boldsymbol{\varepsilon}(\boldsymbol{u})\right) \cdot \boldsymbol{\varepsilon}_{\boldsymbol{y}}(\boldsymbol{v}) d y d x \\
& +\int_{\Omega_{d}} \int_{\boldsymbol{V} \backslash \boldsymbol{I}} \mathrm{A}\left(\boldsymbol{\varepsilon}_{\boldsymbol{y}}\left(\boldsymbol{u}^{1}\right)+\boldsymbol{\varepsilon}(\boldsymbol{u})+\boldsymbol{\varepsilon}\left(\delta \boldsymbol{e}_{3}\right)+\boldsymbol{\varepsilon}_{\boldsymbol{x}}\left(\omega \boldsymbol{e}_{3} \wedge \boldsymbol{y}\right)\right) \cdot \boldsymbol{\varepsilon}_{\boldsymbol{y}}(\boldsymbol{v}) d y d x .
\end{aligned}
$$

Assuming at this stage that the fields $\boldsymbol{u}, \delta$ and $\omega$ are known, (30) will allow us to determine $\boldsymbol{u}^{1}$ in terms of the gradient of $\boldsymbol{u}, \delta$ and $\omega$. For that, we have still to discriminate between the domains $\Omega_{c}$ and $\Omega_{d}$.

(1) Let us first choose $\boldsymbol{v}$ such that $\boldsymbol{v}(\boldsymbol{x}, \boldsymbol{y})=\varphi(\boldsymbol{x}) \boldsymbol{w}(\boldsymbol{y})$ with $\varphi \in \mathscr{D}\left(\Omega_{c}\right)$ (the set of indefinitely differentiable functions with compact support in $\Omega_{c}$ ) and $\boldsymbol{w} \in \mathscr{H}_{c}$, where $\mathscr{H}_{c}$ denotes the Hilbert space of vector fields which are $\boldsymbol{V}$-periodic and whose components are in $H^{1}(\boldsymbol{V})$; i.e.,

$$
\mathscr{H}_{c}=\left\{\boldsymbol{w} \in H^{1}\left(\boldsymbol{V} ; \mathbb{R}^{3}\right): \boldsymbol{w} \text { is } \boldsymbol{V} \text {-periodic }\right\} .
$$

Then (30) becomes: at almost all $\boldsymbol{x} \in \Omega_{c}$ and for all $\boldsymbol{w} \in \mathscr{H}_{c}$,

$$
\int_{V} \mathrm{~A}(\boldsymbol{y}) \boldsymbol{\varepsilon}_{\boldsymbol{y}}\left(\boldsymbol{u}^{1}\right)(\boldsymbol{x}, \boldsymbol{y}) \cdot \boldsymbol{\varepsilon}(\boldsymbol{w})(\boldsymbol{y}) d y+\boldsymbol{\varepsilon}(\boldsymbol{u})(\boldsymbol{x}) \cdot \int_{V} \mathrm{~A}(\boldsymbol{y}) \boldsymbol{\varepsilon}(\boldsymbol{w})(\boldsymbol{y}) d y=0 .
$$

Hence, by linearity, $\boldsymbol{u}^{1}$ can read as

$$
\text { for } \boldsymbol{x} \in \Omega_{c}: \quad u_{k}^{1}(\boldsymbol{x}, \boldsymbol{y})=\varepsilon(\boldsymbol{u})_{i j}(\boldsymbol{x}) \chi_{k}^{i j}(\boldsymbol{y})+\bar{u}_{k}(\boldsymbol{x}) \text { for all } \boldsymbol{y} \in \boldsymbol{V},
$$

where, for $i, j \in\{1,2,3\}$, the vector fields $\chi^{i j}$ are the elements of $\mathscr{H}_{c}$ solving the so-called cell problems

$$
\int_{V} \mathrm{~A}_{p q r s} \varepsilon\left(\chi^{i j}\right)_{p q} \varepsilon(\boldsymbol{w})_{r s} d y+\int_{V} \mathrm{~A}_{i j r s} \varepsilon(\boldsymbol{w})_{r s} d y=0 \quad \text { for all } \boldsymbol{w} \in \mathscr{H}_{c} .
$$

In (31), $\overline{\boldsymbol{u}}(\boldsymbol{x})$ remains undetermined at this stage.

(2) Let us now choose $\boldsymbol{v}$ such that $\boldsymbol{v}(\boldsymbol{x}, \boldsymbol{y})=\varphi(\boldsymbol{x}) \boldsymbol{w}(\boldsymbol{y})$ with $\varphi \in \mathscr{D}\left(\Omega_{d}\right)$ and $\boldsymbol{w} \in \mathscr{H}_{d}$, where

$$
\mathscr{H}_{d}=\left\{\boldsymbol{w} \in H^{1}\left(\boldsymbol{V} \backslash \boldsymbol{I} ; \mathbb{R}^{3}\right): \boldsymbol{w} \text { is } \boldsymbol{V} \text {-periodic, } \llbracket w_{n} \rrbracket=0 \text { on } \boldsymbol{I}\right\} .
$$


Then (30) becomes: at almost all $\boldsymbol{x} \in \Omega_{d}$ and for all $\boldsymbol{w} \in \mathscr{H}_{d}$,

$$
\begin{aligned}
0=\int_{\boldsymbol{V} \backslash \boldsymbol{I}} \mathrm{A}(\boldsymbol{y}) \boldsymbol{\varepsilon}_{\boldsymbol{y}}\left(\boldsymbol{u}^{1}\right)(\boldsymbol{x}, \boldsymbol{y}) \cdot \boldsymbol{\varepsilon}(\boldsymbol{w})(\boldsymbol{y}) d y \\
\quad+\boldsymbol{\varepsilon}(\boldsymbol{u})(\boldsymbol{x}) \cdot \int_{\boldsymbol{V} \backslash \boldsymbol{I}} \mathrm{A}(\boldsymbol{y}) \boldsymbol{\varepsilon}(\boldsymbol{w})(\boldsymbol{y}) d y+\boldsymbol{\varepsilon}\left(\delta \boldsymbol{e}_{3}\right)(\boldsymbol{x}) \cdot \int_{V_{f}} \mathrm{~A}^{f} \boldsymbol{\varepsilon}(\boldsymbol{w})(\boldsymbol{y}) d y \\
\quad+\boldsymbol{\varepsilon}\left(\omega \boldsymbol{e}_{2}\right)(\boldsymbol{x}) \cdot \int_{\boldsymbol{V}_{f}} y_{1} \mathrm{~A}^{f} \boldsymbol{\varepsilon}(\boldsymbol{w})(\boldsymbol{y}) d y-\boldsymbol{\varepsilon}\left(\omega \boldsymbol{e}_{1}\right)(\boldsymbol{x}) \cdot \int_{V_{f}} y_{2} \mathrm{~A}^{f} \boldsymbol{\varepsilon}(\boldsymbol{w})(\boldsymbol{y}) d y .
\end{aligned}
$$

Hence, by linearity, $\boldsymbol{u}^{1}$ can read as

for $\boldsymbol{x} \in \Omega_{d}: \quad \boldsymbol{u}^{1}(\boldsymbol{x}, \boldsymbol{y})=\varepsilon(\boldsymbol{u})_{i j}(\boldsymbol{x}) \xi^{i j}(\boldsymbol{y})+\frac{\partial \delta}{\partial x_{i}}(\boldsymbol{x}) \boldsymbol{D}^{i}(\boldsymbol{y})+\frac{\partial \omega}{\partial x_{i}}(\boldsymbol{x}) \boldsymbol{W}^{i}(\boldsymbol{y})+\overline{\boldsymbol{u}}(\boldsymbol{x}, \boldsymbol{y})$$$
\text { for all } \boldsymbol{y} \in \boldsymbol{V} \backslash \boldsymbol{I} \text {, }
$$

where $\overline{\boldsymbol{u}}(\boldsymbol{x}, \cdot)$ is an element of $\mathscr{R}_{d}$ that remains undetermined at this stage, and the vector fields $\boldsymbol{\xi}^{i j}, \boldsymbol{D}^{i}$ and $\boldsymbol{W}^{i}$, for $i, j \in\{1,2,3\}$, are the elements of $\mathscr{H}_{d}$ solving the following new cell problems:

$$
\begin{array}{r}
\int_{\boldsymbol{V} \backslash \boldsymbol{I}} \mathrm{A}_{p q r s} \varepsilon\left(\boldsymbol{\xi}^{i j}\right)_{p q} \varepsilon(\boldsymbol{w})_{r s} d y+\int_{\boldsymbol{V} \backslash \boldsymbol{I}} \mathrm{A}_{i j r s} \varepsilon(\boldsymbol{w})_{r s} d y=0, \\
\int_{\boldsymbol{V} \backslash \boldsymbol{I}} \mathrm{A}_{p q r s} \varepsilon\left(\boldsymbol{D}^{i}\right)_{p q} \varepsilon(\boldsymbol{w})_{r s} d y+\int_{\boldsymbol{V}_{f}} \mathrm{~A}_{3 i r s}^{f} \varepsilon(\boldsymbol{w})_{r s} d y=0, \\
\int_{\boldsymbol{V} \backslash \boldsymbol{I}} \mathrm{A}_{p q r s} \varepsilon\left(\boldsymbol{W}^{i}\right)_{p q} \varepsilon(\boldsymbol{w})_{r s} d y+\int_{\boldsymbol{V}_{f}}\left(\boldsymbol{e}_{3} \wedge \boldsymbol{y}\right) \cdot \boldsymbol{e}_{q} \mathrm{~A}_{i q r s}^{f} \varepsilon(\boldsymbol{w})_{r s} d y=0 .
\end{array}
$$

In (34)-(36) equality holds for all $\boldsymbol{w} \in \mathscr{H}_{d}$.

Let us study each of these cell problems.

- Each $\chi^{i j}$ is uniquely determined up to a translation which can be fixed by imposing that $\int_{V} \chi^{i j} d y=\mathbf{0}$. It corresponds to the microscopic response of the representative volume element submitted to the macroscopic strain tensor $\boldsymbol{e}_{i} \otimes_{s} \boldsymbol{e}_{j}$. In other words, the $\chi^{i j}$ are given by the classical microscopic problems appearing in the homogenization theory [Allaire 1992; Bensoussan et al. 1978]. By virtue of the symmetries of the rigidity tensors $\mathrm{A}^{f}$ and $\mathrm{A}^{m}$, one has $\chi^{i j}=\chi^{j i}$ and hence there exist exactly six independent cell problems. Since the periodicity is twodimensional and since the fibers and the matrix are isotropic, all the $\chi^{i j}$ enjoy some general properties. For instance,

$$
\chi_{3}^{\alpha \beta}=\chi_{3}^{33}=\chi_{\beta}^{\alpha 3}=0 \quad \text { for all } \alpha, \beta \in\{1,2\} .
$$

Additional symmetry properties appear when the cell itself enjoys additional symmetries [Léné 1984]. The practical determination of the $\chi^{i j}$ requires some numerical computation. 
- All preceding comments on the $\chi^{i j}$ remain true for the $\xi^{i j}$ (except that $\xi^{i j}$ is uniquely determined up to an element of $\mathscr{R}_{d}$ ). Note however that $\xi^{i j}$ differs (in general) from $\chi^{i j}$ because of the possibility of a tangential discontinuity of $\xi^{i j}$ on $\boldsymbol{I}$. A consequence of this additional degree of freedom is that the shear stress associated with $\xi^{i j}$ necessarily vanishes on $\boldsymbol{I}$ while this is not in general the case for $\chi^{i j}$.

- The fields $\boldsymbol{D}^{1}$ and $\boldsymbol{D}^{2}$ can be obtained in a closed form. Specifically, one gets

$$
\text { for } \alpha \in\{1,2\}: \quad \boldsymbol{D}^{\alpha}(y)=\left\{\begin{array}{cl}
\mathbf{0}, & \boldsymbol{y} \in \boldsymbol{V}_{m}, \\
-y_{\alpha} \boldsymbol{e}_{3}, & \boldsymbol{y} \in \boldsymbol{V}_{f},
\end{array} \quad+\text { an arbitrary element of } \mathscr{R}_{d} .\right.
$$

The verification is straightforward and left to the reader. On the other hand, $\boldsymbol{D}^{3}$ cannot be obtained in a closed form (except if $\lambda_{f}=0$ ) but can be simplified. Indeed, as for the $\xi^{i j}$, by virtue of the isotropy of the fibers and the matrix, one gets that $D_{3}^{3}=0$ and finally the problem for $\boldsymbol{D}^{3}$ can read as

$$
\begin{aligned}
& \int_{\boldsymbol{V} \backslash \boldsymbol{I}} \lambda \varepsilon\left(\boldsymbol{D}^{3}\right)_{\alpha \alpha} \varepsilon(\boldsymbol{w})_{\beta \beta}+2 \mu \varepsilon\left(\boldsymbol{D}^{3}\right)_{\alpha \beta} \varepsilon(\boldsymbol{w})_{\alpha \beta} d y+\int_{\boldsymbol{V}_{f}} \lambda_{f} \varepsilon(\boldsymbol{w})_{\beta \beta} d y=0 \\
& \text { for all } \boldsymbol{w} \in \mathscr{H}_{d} \text {. }
\end{aligned}
$$

It corresponds to the response of the cell when the fiber is submitted to a macroscopic longitudinal stretching $\boldsymbol{e}_{3} \otimes \boldsymbol{e}_{3}$ while the matrix is macroscopically unstrained. That response is not trivial because of the contact between the fiber and the matrix. This contact implies the existence of a normal stress $\sigma_{n n}$ at the interface $\boldsymbol{I}$ which induces a deformation of the matrix.

- All the fields $\boldsymbol{W}^{i}$ can be obtained in a closed form. Let us first show that

$$
\boldsymbol{W}^{3} \in \mathscr{R}_{d} .
$$

Indeed, the integral over $\boldsymbol{V}_{f}$ in (36) for $i=3$ vanishes as proved below:

$$
\begin{aligned}
\int_{V_{f}}\left(\boldsymbol{e}_{3} \wedge \boldsymbol{y}\right) \cdot \boldsymbol{e}_{\beta} \mathrm{A}_{3 \beta k l}^{f} \varepsilon(\boldsymbol{w})_{k l} d y & =\int_{V_{f}} \mu_{f}\left(\boldsymbol{e}_{3} \wedge \boldsymbol{y}\right) \cdot \boldsymbol{e}_{\beta} \frac{\partial w_{3}}{\partial y_{\beta}} d y \\
& =-\int_{V_{f}} \mu_{f}\left(\boldsymbol{e}_{3} \wedge \boldsymbol{e}_{\beta}\right) \cdot \boldsymbol{e}_{\beta} w_{3} d y+\int_{\boldsymbol{I}} \mu_{f}\left(\boldsymbol{e}_{3} \wedge \boldsymbol{y}\right) \cdot \boldsymbol{n} w_{3} d s \\
& =0 .
\end{aligned}
$$

The last equality above is due to the fact that $\boldsymbol{n}=\boldsymbol{y} / R$ on $\boldsymbol{I}$. Inserting this property and taking $\boldsymbol{w}=\boldsymbol{W}^{3}$ in (36) for $i=3$ leads to

$$
\int_{\boldsymbol{V} \backslash \boldsymbol{I}} \mathrm{A} \boldsymbol{\varepsilon}\left(\boldsymbol{W}^{3}\right) \cdot \boldsymbol{\varepsilon}\left(\boldsymbol{W}^{3}\right) d y=0 .
$$

Therefore $\boldsymbol{\varepsilon}\left(\boldsymbol{W}^{3}\right)=\mathbf{0}$ which is the desired result. Since the undetermined element of $\mathscr{R}_{d}$ does not play any role, one can consider that $\boldsymbol{W}^{3}=\mathbf{0}$. Note that this property 
holds true because the fiber has a circular section and is isotropic.

Let us now verify that $\boldsymbol{W}^{1}$ and $\boldsymbol{W}^{2}$ are given by for $\alpha \in\{1,2\}: \boldsymbol{W}^{\alpha}(y)=\left\{\begin{array}{cl}\mathbf{0}, & \boldsymbol{y} \in \boldsymbol{V}_{m}, \\ -y_{\alpha} \boldsymbol{e}_{3} \wedge \boldsymbol{y}, & \boldsymbol{y} \in \boldsymbol{V}_{f},\end{array} \quad+\right.$ an arbitrary element of $\mathscr{R}_{d}$. (40) Let us first remark that $\llbracket \boldsymbol{W}^{\alpha} \rrbracket \cdot \boldsymbol{n}=0$ on $\boldsymbol{I}$ because $\left(\boldsymbol{e}_{3} \wedge \boldsymbol{y}\right) \cdot \boldsymbol{n}=0$. Hence $\boldsymbol{W}^{\alpha} \in \mathscr{H}_{d}$. Let us now calculate the strain field $\boldsymbol{\varepsilon}\left(\boldsymbol{W}^{\alpha}\right)$ for $\alpha \in\{1,2\}$ :

$$
2 \varepsilon\left(\boldsymbol{W}^{\alpha}\right)_{p q}=-\left(\boldsymbol{e}_{3} \wedge \boldsymbol{y}\right) \cdot \boldsymbol{e}_{p} \delta_{\alpha q}-\left(\boldsymbol{e}_{3} \wedge \boldsymbol{y}\right) \cdot \boldsymbol{e}_{q} \delta_{\alpha p} \quad \text { for all } p, q \in\{1,2,3\} .
$$

Therefore, one gets $\mathrm{A}_{p q r s}^{f} \varepsilon\left(\boldsymbol{W}^{\alpha}\right)_{p q}=-\left(\boldsymbol{e}_{3} \wedge \boldsymbol{y}\right) \cdot \boldsymbol{e}_{q} \mathrm{~A}_{\alpha q r s}^{f}$, from which one easily deduces that (36) is satisfied for $i=\alpha$.

3.5. The form of $\sigma^{0}$. The form of the leading term $\sigma^{0}$ of the stress field is obtained via the constitutive relations (8) and the strain expansion (23). Specifically, one gets

$$
\sigma^{0}(x, y)=\mathrm{A}(y)\left(\varepsilon_{x}\left(u^{0}\right)(x, y)+\varepsilon_{y}\left(u^{1}\right)(x, y)\right) .
$$

Let us discriminate once more between the domains $\Omega_{c}$ and $\Omega_{d}$ to obtain the stress field $\sigma^{0}$ in terms of the generalized strain fields $\boldsymbol{\varepsilon}(\boldsymbol{u}), \nabla \delta, \nabla \omega$ and of the microscopic strain fields associated with the solutions of the cell problems.

(1) For $x \in \Omega_{c}$. By virtue of (27) and (31), one gets

$$
\boldsymbol{\sigma}^{0}(\boldsymbol{x}, \boldsymbol{y})=\mathrm{A}(\boldsymbol{y})\left(\boldsymbol{\varepsilon}(\boldsymbol{u})(\boldsymbol{x})+\varepsilon(\boldsymbol{u})_{i j}(\boldsymbol{x}) \boldsymbol{\varepsilon}\left(\chi^{i j}\right)(\boldsymbol{y})\right),
$$

which is the usual expression of the stress distribution given by the homogenization theory. Of course, all cell problems give a contribution to that stress distribution.

(2) For $\boldsymbol{x} \in \Omega_{d}$. By virtue of (28) and (33), one gets, for all $\boldsymbol{y} \in \boldsymbol{V} \backslash \boldsymbol{I}$,

$$
\boldsymbol{\sigma}^{0}(\boldsymbol{x}, \boldsymbol{y})=\mathrm{A}(\boldsymbol{y})\left(\boldsymbol{\varepsilon}(\boldsymbol{u})(\boldsymbol{x})+\varepsilon(\boldsymbol{u})_{i j}(\boldsymbol{x}) \boldsymbol{\varepsilon}\left(\boldsymbol{\xi}^{i j}\right)(\boldsymbol{y})\right)+\frac{\partial \delta}{\partial x_{i}}(\boldsymbol{x}) \boldsymbol{S}^{i}(\boldsymbol{y})+\frac{\partial \omega}{\partial x_{i}}(\boldsymbol{x}) \boldsymbol{T}^{i}(\boldsymbol{y}),
$$

with

$$
\begin{aligned}
& S_{r s}^{i}(\boldsymbol{y})=\left\{\begin{array}{cl}
\mathrm{A}_{p q r s}^{m} \varepsilon\left(\boldsymbol{D}^{i}\right)_{p q}(\boldsymbol{y}) & \text { if } \boldsymbol{y} \in \boldsymbol{V}_{m}, \\
\mathrm{~A}_{p q r s}^{f} \varepsilon\left(\boldsymbol{D}^{i}\right)_{p q}(\boldsymbol{y})+\mathrm{A}_{3 i r s}^{f} & \text { if } \boldsymbol{y} \in \boldsymbol{V}_{f}
\end{array}\right. \\
& T_{r s}^{i}(\boldsymbol{y})=\left\{\begin{array}{cc}
\mathrm{A}_{p q r s}^{m} \varepsilon\left(\boldsymbol{W}^{i}\right)_{p q}(\boldsymbol{y}) & \text { if } \boldsymbol{y} \in \boldsymbol{V}_{m}, \\
\mathrm{~A}_{p q r s}^{f} \varepsilon\left(\boldsymbol{W}^{i}\right)_{p q}(\boldsymbol{y})+\mathrm{A}_{i q r s}^{f}\left(\boldsymbol{e}_{3} \wedge \boldsymbol{y}\right) \cdot \boldsymbol{e}_{q} & \text { if } \boldsymbol{y} \in \boldsymbol{V}_{f} .
\end{array}\right.
\end{aligned}
$$

Moreover, (37) gives $\boldsymbol{S}^{\alpha}=\mathbf{0}$ and (40) gives $\boldsymbol{T}^{\alpha}=\mathbf{0}$ for $\alpha \in\{1,2\}$. In other words the cell problems associated with $\partial \delta / \partial x_{\alpha}$ or with $\partial \omega / \partial x_{\alpha}$ induce no stress. Since $\boldsymbol{W}^{3}$ vanishes, $\boldsymbol{T}^{3}$ reads as

$$
\boldsymbol{T}^{3}(\boldsymbol{y})=\left\{\begin{array}{cl}
\mathbf{0} & \text { if } \boldsymbol{y} \in \boldsymbol{V}_{m}, \\
2 \mu_{f}\left(-y_{2} \boldsymbol{e}_{3} \otimes_{s} \boldsymbol{e}_{1}+y_{1} \boldsymbol{e}_{3} \otimes_{s} \boldsymbol{e}_{2}\right) & \text { if } \boldsymbol{y} \in \boldsymbol{V}_{f} .
\end{array}\right.
$$


Note that this stress distribution corresponds to that given by a torsion of a cylinder with a circular cross-section. The only nonzero component is the orthoradial one $\sigma_{3 \theta}$ which is proportional to $r$, the distance to the axis. Moreover, there is no interaction with the matrix.

On the other hand, $S^{3}$ cannot be obtained in a closed form, but can be simplified by using (38):

$$
\begin{aligned}
& S_{\alpha \beta}^{3}(\boldsymbol{y})=\left\{\begin{array}{cc}
\lambda_{m} \varepsilon_{\gamma \gamma}\left(\boldsymbol{D}^{3}\right)(\boldsymbol{y}) \delta_{\alpha \beta}+2 \mu_{m} \varepsilon_{\alpha \beta}\left(\boldsymbol{D}^{3}\right)(\boldsymbol{y}) & \text { if } \boldsymbol{y} \in \boldsymbol{V}_{m}, \\
\lambda_{f}\left(1+\varepsilon_{\gamma \gamma}\left(\boldsymbol{D}^{3}\right)(\boldsymbol{y})\right) \delta_{\alpha \beta}+2 \mu_{f} \varepsilon_{\alpha \beta}\left(\boldsymbol{D}^{3}\right)(\boldsymbol{y}) & \text { if } \boldsymbol{y} \in \boldsymbol{V}_{f},
\end{array}\right. \\
& S_{33}^{3}(\boldsymbol{y})=\left\{\begin{array}{cc}
\lambda_{m} \varepsilon_{\gamma \gamma}\left(\boldsymbol{D}^{3}\right)(\boldsymbol{y}) & \text { if } \boldsymbol{y} \in \boldsymbol{V}_{m}, \\
\lambda_{f}\left(1+\varepsilon_{\gamma \gamma}\left(\boldsymbol{D}^{3}\right)(\boldsymbol{y})\right)+2 \mu_{f} & \text { if } \boldsymbol{y} \in \boldsymbol{V}_{f},
\end{array}\right.
\end{aligned}
$$

and $S_{\alpha 3}^{3}=0$ in $\boldsymbol{V}_{f} \cup \boldsymbol{V}_{m}$. As it was already noted, there is an interaction between the fiber and the matrix because of the contact assumption.

Finally, $\boldsymbol{\sigma}^{0}(\boldsymbol{x}, \cdot)$ can read in $\boldsymbol{V} \backslash \boldsymbol{I}$ as

$\boldsymbol{\sigma}^{0}(\boldsymbol{x}, \boldsymbol{y})=\mathrm{A}(\boldsymbol{y})\left(\boldsymbol{\varepsilon}(\boldsymbol{u})(\boldsymbol{x})+\varepsilon(\boldsymbol{u})_{i j}(\boldsymbol{x}) \boldsymbol{\varepsilon}\left(\boldsymbol{\xi}^{i j}\right)(\boldsymbol{y})\right)+\frac{\partial \delta}{\partial x_{3}}(\boldsymbol{x}) \boldsymbol{S}^{3}(\boldsymbol{y})+\frac{\partial \omega}{\partial x_{3}}(\boldsymbol{x}) \boldsymbol{T}^{3}(\boldsymbol{y})$,

which includes the contribution of the longitudinal stretching and the torsion of the fibers.

3.6. The macroscopic problem. To obtain the problem which gives the macroscopic fields $\boldsymbol{u}, \delta$ and $\omega$, we choose a displacement field $\boldsymbol{v}$ in (26) of the same type as $\boldsymbol{u}^{0}$, i.e., such that $\boldsymbol{\varepsilon}_{\boldsymbol{y}}(\boldsymbol{v})=\mathbf{0}$. Specifically, one sets

$$
\boldsymbol{v}^{*}(\boldsymbol{x}, \boldsymbol{y})=\left\{\begin{array}{cl}
\boldsymbol{u}^{*}(\boldsymbol{x}) & \text { in }\left(\Omega_{c} \times V\right) \cup\left(\Omega_{d} \times \boldsymbol{V}_{m}\right), \\
\boldsymbol{u}^{*}(\boldsymbol{x})+\delta^{*}(\boldsymbol{x}) \boldsymbol{e}_{3}+\omega^{*}(\boldsymbol{x}) \boldsymbol{e}_{3} \wedge \boldsymbol{y} & \text { in } \Omega_{d} \times \boldsymbol{V}_{f}
\end{array}\right.
$$

and inserts such a $\boldsymbol{v}^{*}$ into (26). Then the terms in $\boldsymbol{\varepsilon}_{\boldsymbol{y}}\left(\boldsymbol{u}^{2}\right)+\boldsymbol{\varepsilon}_{\boldsymbol{x}}\left(\boldsymbol{u}^{1}\right)$ disappear because $\boldsymbol{\varepsilon}_{\boldsymbol{y}}(\boldsymbol{v})=0$. By virtue of (41), (26) becomes

$$
\begin{aligned}
& \int_{\Omega} \int_{V} \boldsymbol{\sigma}^{0}(\boldsymbol{x}, \boldsymbol{y}) \cdot \boldsymbol{\varepsilon}\left(\boldsymbol{u}^{*}\right)(\boldsymbol{x}) d y d x \\
& \quad+\int_{\Omega_{d}} \int_{V_{f}} \boldsymbol{\sigma}^{0}(\boldsymbol{x}, \boldsymbol{y}) \cdot\left(\boldsymbol{\varepsilon}\left(\delta^{*} \boldsymbol{e}_{3}\right)(\boldsymbol{x})+\boldsymbol{\varepsilon}\left(\omega^{*} \boldsymbol{e}_{3} \wedge \boldsymbol{e}_{\alpha}\right)(\boldsymbol{x}) y_{\alpha}\right) d y d x \\
& =\int_{\Omega} \int_{V} \rho(\boldsymbol{y}) \boldsymbol{g}(\boldsymbol{x}) \cdot \boldsymbol{u}^{*}(\boldsymbol{x}) d y d x+\int_{\Omega_{d}} \int_{V_{f}} \rho_{f}\left(g_{3}(\boldsymbol{x}) \delta^{*}(\boldsymbol{x})+\left(\boldsymbol{e}_{3} \wedge \boldsymbol{y}\right) \cdot \boldsymbol{g}(\boldsymbol{x}) \omega^{*}(\boldsymbol{x})\right) d y d x \\
& +\int_{\Gamma_{s}} \int_{\boldsymbol{V}} \boldsymbol{F}(\boldsymbol{x}) \cdot \boldsymbol{u}^{*}(\boldsymbol{x}) d y d \Gamma+\int_{\Gamma_{s}} \int_{V_{f}}\left(F_{3}(\boldsymbol{x}) \delta^{*}(\boldsymbol{x})+\left(\boldsymbol{e}_{3} \wedge \boldsymbol{y}\right) \cdot \boldsymbol{F}(\boldsymbol{x}) \omega^{*}(\boldsymbol{x})\right) d y d \Gamma .
\end{aligned}
$$

Let us denote by $\langle\varphi\rangle$ the mean value of $\varphi$ over the cell $\boldsymbol{V}$ :

$$
\langle\varphi\rangle=\frac{1}{|\boldsymbol{V}|} \int_{\boldsymbol{V}} \varphi(\boldsymbol{y}) d y, \quad\langle\varphi\rangle(\boldsymbol{x})=\frac{1}{|\boldsymbol{V}|} \int_{\boldsymbol{V}} \varphi(\boldsymbol{x}, \boldsymbol{y}) d y,
$$


and by $\langle\varphi\rangle_{f}$ (respectively, $\langle\varphi\rangle_{m}$ ) the mean value over the whole cell $\boldsymbol{V}$ of the field $\varphi$ only defined in or restricted to $\boldsymbol{V}_{f}$ (respectively, $\boldsymbol{V}_{m}$ ); i.e.,

$$
\langle\varphi\rangle_{f, m}=\frac{1}{|\boldsymbol{V}|} \int_{\boldsymbol{V}_{f, m}} \varphi(\boldsymbol{y}) d y, \quad\langle\varphi\rangle_{f, m}(\boldsymbol{x})=\frac{1}{|\boldsymbol{V}|} \int_{\boldsymbol{V}_{f, m}} \varphi(\boldsymbol{x}, \boldsymbol{y}) d y .
$$

Recalling that the center of the fiber is taken as the origin of the $\boldsymbol{y}$-coordinates, one has $\int_{\boldsymbol{V}_{f}} \boldsymbol{y} d y=\mathbf{0}$. Accordingly, after easy calculations, (51) can read as

$$
\begin{aligned}
& \int_{\Omega_{c}}\left\langle\boldsymbol{\sigma}^{0}\right\rangle \cdot \boldsymbol{\varepsilon}\left(\boldsymbol{u}^{*}\right) d x+\int_{\Omega_{d}}\left(\left\langle\boldsymbol{\sigma}^{0}\right\rangle \cdot \boldsymbol{\varepsilon}\left(\boldsymbol{u}^{*}\right)+\left\langle\boldsymbol{\sigma}^{0}\right\rangle_{f} \boldsymbol{e}_{3} \cdot \nabla \delta^{*}+\left\langle y_{\alpha} \boldsymbol{\sigma}^{0}\right\rangle_{f} \cdot \boldsymbol{\varepsilon}\left(\omega^{*} \boldsymbol{e}_{3} \wedge \boldsymbol{e}_{\alpha}\right)\right) d x \\
& =\int_{\Omega_{c}}\langle\rho\rangle \boldsymbol{g} \cdot \boldsymbol{u}^{*} d x+\int_{\Omega_{d}}\left(\langle\rho\rangle \boldsymbol{g} \cdot \boldsymbol{u}^{*}+\rho_{f} V_{f} g_{3} \delta^{*}\right) d x+\int_{\Gamma_{s}}\left(\boldsymbol{F} \cdot \boldsymbol{u}^{*}+V_{f} F_{3} \delta^{*}\right) d \Gamma,
\end{aligned}
$$

where $V_{f}$ denotes the volume fraction of the fibers; i.e.,

$$
V_{f}=\frac{\left|\boldsymbol{V}_{f}\right|}{|\boldsymbol{V}|}, \quad V_{m}=1-V_{f} .
$$

Remark 3. Let us note that $\omega^{*}$ does not appear in the right-hand side of (54). This is due to the assumption made on the applied forces, specifically that both the specific bulk forces $\boldsymbol{g}$ and the surface forces $\boldsymbol{F}$ do not depend on $\boldsymbol{y}$, and on the choice of the center of the fiber as the origin of the $\boldsymbol{y}$ coordinates.

Let us examine each term of the left-hand side of (54).

- For $\boldsymbol{x} \in \Omega_{c}$, by virtue of $(42),\left\langle\boldsymbol{\sigma}^{0}\right\rangle(\boldsymbol{x})$ reads as

$$
\left\langle\boldsymbol{\sigma}^{0}\right\rangle(\boldsymbol{x})=\mathrm{A}^{c} \boldsymbol{\varepsilon}(\boldsymbol{u})(\boldsymbol{x}),
$$

where $A^{c}$ denotes the (classical) homogenized stiffness tensor of the (perfectly bonded) composite; i.e.,

$$
\mathrm{A}_{i j k l}^{c}=\left\langle\mathrm{A}_{i j k l}+\mathrm{A}_{i j p q} \varepsilon\left(\chi^{k l}\right)_{p q}\right\rangle=\left\langle\mathrm{A}_{i j k l}-\mathrm{A} \boldsymbol{\varepsilon}\left(\chi^{i j}\right) \cdot \boldsymbol{\varepsilon}\left(\chi^{k l}\right)\right\rangle .
$$

The last equality above is obtained by using (32) with $\boldsymbol{w}=\chi^{k l}$. It allows us to check that $\mathrm{A}^{c}$ has the major symmetry $\mathrm{A}_{i j k l}^{c}=\mathrm{A}_{k l i j}^{c}$.

- For $\boldsymbol{x} \in \Omega_{d}$, by virtue of (49), $\left\langle\boldsymbol{\sigma}^{0}\right\rangle(\boldsymbol{x})$ reads as

$$
\left\langle\boldsymbol{\sigma}^{0}\right\rangle(\boldsymbol{x})=\mathrm{A}^{d} \boldsymbol{\varepsilon}(\boldsymbol{u})(\boldsymbol{x})+\left\langle\boldsymbol{S}^{3}\right\rangle \frac{\partial \delta}{\partial x_{3}}(\boldsymbol{x})+\left\langle\boldsymbol{T}^{3}\right\rangle \frac{\partial \omega}{\partial x_{3}}(\boldsymbol{x}),
$$

where $\mathrm{A}^{d}$ denotes the homogenized stiffness tensor of the debonded composite; i.e.,

$$
\mathrm{A}_{i j k l}^{d}=\left\langle\mathrm{A}_{i j k l}+\mathrm{A}_{i j p q} \varepsilon\left(\xi^{k l}\right)_{p q}\right\rangle=\left\langle\mathrm{A}_{i j k l}-\mathrm{A} \boldsymbol{\varepsilon}\left(\xi^{i j}\right) \cdot \boldsymbol{\varepsilon}\left(\boldsymbol{\xi}^{k l}\right)\right\rangle .
$$

The last equality above is obtained by using (34) with $\boldsymbol{w}=\boldsymbol{\xi}^{k l}$ and implies that $\mathrm{A}_{i j k l}^{d}=\mathrm{A}_{k l i j}^{d}$ for all $i, j, k, l$. The tensor $\mathrm{A}^{d}$ will be compared to the tensor $\mathrm{A}^{c}$ in 
the next section. Then, using (46) and the fact that $\langle\boldsymbol{y}\rangle_{f}=\mathbf{0}$, one gets $\left\langle\boldsymbol{T}^{3}\right\rangle=\mathbf{0}$ and finally

$$
\left\langle\boldsymbol{\sigma}^{0}\right\rangle(\boldsymbol{x})=\mathrm{A}^{d} \boldsymbol{\varepsilon}(\boldsymbol{u})(\boldsymbol{x})+\left\langle\boldsymbol{S}^{3}\right\rangle \frac{\partial \delta}{\partial x_{3}}(\boldsymbol{x}) .
$$

- For $\boldsymbol{x} \in \Omega_{d}$, using (49), the component $i$ of $\left\langle\boldsymbol{\sigma}^{0}\right\rangle_{f} \boldsymbol{e}_{3}(\boldsymbol{x})$ reads as

$$
\left\langle\sigma_{3 i}^{0}\right\rangle_{f}(\boldsymbol{x})=\left\langle\mathrm{A}_{3 i k l}^{f}+\mathrm{A}_{3 i r s}^{f} \varepsilon\left(\boldsymbol{\xi}^{k l}\right)_{r s}\right\rangle_{f} \varepsilon(\boldsymbol{u})_{k l}(\boldsymbol{x})+\left\langle S_{3 i}^{3}\right\rangle_{f} \frac{\partial \delta}{\partial x_{3}}(\boldsymbol{x})+\left\langle T_{3 i}^{3}\right\rangle_{f} \frac{\partial \omega}{\partial x_{3}}(\boldsymbol{x}) .
$$

Let us first show that

$$
\left\langle\mathrm{A}_{3 i k l}^{f}+\mathrm{A}_{3 i r s}^{f} \varepsilon\left(\xi^{k l}\right)_{r s}\right\rangle_{f}=\left\langle S_{k l}^{3}\right\rangle \delta_{i 3} .
$$

Considering (35) with $\boldsymbol{w}=\boldsymbol{\xi}^{k l}$ gives

$$
\left\langle A \boldsymbol{\varepsilon}\left(\boldsymbol{D}^{i}\right) \cdot \boldsymbol{\varepsilon}\left(\boldsymbol{\xi}^{k l}\right)\right\rangle+\left\langle\mathrm{A}_{3 i r s}^{f} \varepsilon\left(\boldsymbol{\xi}^{k l}\right)_{r s}\right\rangle_{f}=0 .
$$

Considering (34) with $k l$ instead of $i j$ and setting $\boldsymbol{w}=\boldsymbol{D}^{i}$ give

$$
\left\langle A \boldsymbol{\varepsilon}\left(\boldsymbol{D}^{i}\right) \cdot \boldsymbol{\varepsilon}\left(\boldsymbol{\xi}^{k l}\right)\right\rangle+\left\langle\mathrm{A}_{k l r s} \varepsilon\left(\boldsymbol{D}^{i}\right)_{r s}\right\rangle=0 .
$$

Therefore $\left\langle\mathrm{A}_{3 i r s}^{f} \varepsilon\left(\boldsymbol{\xi}^{k l}\right)_{r s}\right\rangle_{f}=\left\langle\mathrm{A}_{k l r s} \varepsilon\left(\boldsymbol{D}^{i}\right)_{r s}\right\rangle$ and hence

$$
\left\langle\mathrm{A}_{3 i k l}^{f}+\mathrm{A}_{3 i r s}^{f} \varepsilon\left(\boldsymbol{\xi}^{k l}\right)_{r s}\right\rangle_{f}=\left\langle\mathrm{A}_{k l r s} \varepsilon\left(\boldsymbol{D}^{i}\right)_{r s}\right\rangle+\left\langle\mathrm{A}_{3 i k l}^{f}\right\rangle_{f}=\left\langle S_{k l}^{i}\right\rangle,
$$

where the last equality is a direct consequence of the definition (44) of $\boldsymbol{S}^{i}$. Since $\boldsymbol{S}^{\alpha}=\mathbf{0}$, one gets (60).

Recalling now that $S_{3 \alpha}^{3}=0$ and $\left\langle\boldsymbol{T}^{3}\right\rangle_{f}=\left\langle\boldsymbol{T}^{3}\right\rangle=\mathbf{0}$, one finally obtains

$$
\left\langle\boldsymbol{\sigma}^{0}\right\rangle_{f} \boldsymbol{e}_{3}(\boldsymbol{x})=\left\langle\boldsymbol{S}^{3}\right\rangle \cdot \boldsymbol{\varepsilon}(\boldsymbol{u})(\boldsymbol{x}) \boldsymbol{e}_{3}+\left\langle S_{33}^{3}\right\rangle_{f} \frac{\partial \delta}{\partial x_{3}}(\boldsymbol{x}) \boldsymbol{e}_{3} .
$$

- The last term in the left-hand side of (54) can also read as

$$
\left\langle y_{\alpha} \boldsymbol{\sigma}^{0}\right\rangle_{f}(\boldsymbol{x}) \cdot \boldsymbol{\varepsilon}\left(\omega^{*} \boldsymbol{e}_{3} \wedge \boldsymbol{e}_{\alpha}\right)(\boldsymbol{x})=\left\langle\left(\boldsymbol{e}_{3} \wedge \boldsymbol{y}\right) \cdot \boldsymbol{e}_{q} \sigma_{q i}^{0}\right\rangle_{f}(\boldsymbol{x}) \frac{\partial \omega^{*}}{\partial x_{i}}(\boldsymbol{x}) .
$$

Using (49), one gets

$$
\begin{aligned}
\left\langle\left(\boldsymbol{e}_{3} \wedge \boldsymbol{y}\right) \cdot \boldsymbol{e}_{q} \sigma_{q i}^{0}\right\rangle_{f}(\boldsymbol{x})=\left\langle\left(\boldsymbol{e}_{3} \wedge \boldsymbol{y}\right) \cdot \boldsymbol{e}_{q}\left(\mathrm{~A}_{q i k l}^{f}+\mathrm{A}_{q i r s}^{f} \varepsilon\left(\boldsymbol{\xi}^{k l}\right)_{r s}\right)\right\rangle_{f} \varepsilon(\boldsymbol{u})_{k l}(\boldsymbol{x}) & \\
& +\left\langle\left(\boldsymbol{e}_{3} \wedge \boldsymbol{y}\right) \cdot \boldsymbol{e}_{q} S_{q i}^{3}\right\rangle_{f} \frac{\partial \delta}{\partial x_{3}}(\boldsymbol{x})+\left\langle\left(\boldsymbol{e}_{3} \wedge \boldsymbol{y}\right) \cdot \boldsymbol{e}_{q} T_{q i}^{3}\right\rangle_{f} \frac{\partial \omega}{\partial x_{3}}(\boldsymbol{x}) .
\end{aligned}
$$

Let us calculate the three effective coefficients appearing in the right side.

We first show that $\left\langle\left(\boldsymbol{e}_{3} \wedge \boldsymbol{y}\right) \cdot \boldsymbol{e}_{q}\left(\mathrm{~A}_{q i k l}^{f}+\mathrm{A}_{\text {qirs }}^{f} \varepsilon\left(\boldsymbol{\xi}^{k l}\right)_{r s}\right)\right\rangle_{f}=0$. First,

$$
\left\langle\left(\boldsymbol{e}_{3} \wedge \boldsymbol{y}\right) \cdot \boldsymbol{e}_{q} \mathrm{~A}_{q i k l}^{f}\right\rangle_{f}=\left(\boldsymbol{e}_{3} \wedge\langle\boldsymbol{y}\rangle_{f}\right) \cdot \boldsymbol{e}_{q} \mathrm{~A}_{q i k l}^{f}=0 .
$$

Then, recalling that $\boldsymbol{W}^{3}=\mathbf{0}$ and using (36) with $i=3$ and $\boldsymbol{w}=\boldsymbol{\xi}^{k l}$ give

$$
\left\langle\left(\boldsymbol{e}_{3} \wedge \boldsymbol{y}\right) \cdot \boldsymbol{e}_{q} \mathrm{~A}_{\text {qirs }}^{f} \varepsilon\left(\boldsymbol{\xi}^{k l}\right)_{r s}\right\rangle_{f}=0
$$


and hence the desired result.

Next we show that $\left\langle\left(\boldsymbol{e}_{3} \wedge \boldsymbol{y}\right) \cdot \boldsymbol{e}_{q} S_{q i}^{3}\right\rangle_{f}=0$. By virtue of (44), one has

$$
\left\langle\left(\boldsymbol{e}_{3} \wedge \boldsymbol{y}\right) \cdot \boldsymbol{e}_{q} S_{q i}^{3}\right\rangle_{f}=\left\langle\left(\boldsymbol{e}_{3} \wedge \boldsymbol{y}\right) \cdot \boldsymbol{e}_{q}\left(\mathrm{~A}_{q i 33}^{f}+\mathrm{A}_{q i r s}^{f} \varepsilon\left(\boldsymbol{D}^{3}\right)_{r s}\right)\right\rangle_{f} .
$$

Therefore, one can follow the same procedure as for the first coefficient. First,

$$
\left\langle\left(\boldsymbol{e}_{3} \wedge \boldsymbol{y}\right) \cdot \boldsymbol{e}_{q} \mathrm{~A}_{q i 33}^{f}\right\rangle_{f}=0 .
$$

Then, using (36) with $i=3$ and $\boldsymbol{w}=\boldsymbol{D}^{3}$ give

$$
\left\langle\left(\boldsymbol{e}_{3} \wedge \boldsymbol{y}\right) \cdot \boldsymbol{e}_{q} \mathrm{~A}_{q i r s}^{f} \varepsilon\left(\boldsymbol{D}^{3}\right)_{r s}\right\rangle_{f}=0
$$

and hence the desired result.

For the third effective coefficient, a direct calculation using (46) gives $\left\langle\left(\boldsymbol{e}_{3} \wedge \boldsymbol{y}\right)\right.$. $\left.\boldsymbol{e}_{q} T_{q i}^{3}\right\rangle_{f}=(\pi / 2) \mu_{f} R^{4} \delta_{i 3}$.

Therefore, one finally obtains

$$
\left\langle\left(\boldsymbol{e}_{3} \wedge \boldsymbol{y}\right) \cdot \boldsymbol{e}_{q} \sigma_{q i}^{0}\right\rangle_{f}(\boldsymbol{x})=\frac{\pi R^{4} \mu_{f}}{2|\boldsymbol{V}|} \frac{\partial \omega}{\partial x_{3}}(\boldsymbol{x}) \delta_{i 3} .
$$

Inserting (55), (59), (61) and (62) into (54), the variational equation (54) finally reads as

$$
\begin{gathered}
\int_{\Omega_{c}} A^{c} \boldsymbol{\varepsilon}(\boldsymbol{u}) \cdot \boldsymbol{\varepsilon}\left(\boldsymbol{u}^{*}\right) d x+\int_{\Omega_{d}} \frac{\pi R^{4} \mu_{f}}{2|\boldsymbol{V}|} \frac{\partial \omega}{\partial x_{3}} \frac{\partial \omega^{*}}{\partial x_{3}} d x \\
+\int_{\Omega_{d}}\left(A^{d} \boldsymbol{\varepsilon}(\boldsymbol{u}) \cdot \boldsymbol{\varepsilon}\left(\boldsymbol{u}^{*}\right)+\left\langle\boldsymbol{S}^{3}\right\rangle \cdot\left(\boldsymbol{\varepsilon}(\boldsymbol{u}) \frac{\partial \delta^{*}}{\partial x_{3}}+\frac{\partial \delta}{\partial x_{3}} \boldsymbol{\varepsilon}\left(\boldsymbol{u}^{*}\right)\right)+\left\langle S_{33}^{3}\right\rangle_{f} \frac{\partial \delta}{\partial x_{3}} \frac{\partial \delta^{*}}{\partial x_{3}}\right) d x \\
=\int_{\Omega}\langle\rho\rangle \boldsymbol{g} \cdot \boldsymbol{u}^{*} d x+\int_{\Omega_{d}} \rho_{f} V_{f} g_{3} \delta^{*} d x+\int_{\Gamma_{s}}\left(\boldsymbol{F} \cdot \boldsymbol{u}^{*}+V_{f} F_{3} \delta^{*}\right) d \Gamma
\end{gathered}
$$

The equality (63) must hold for all $\left(\boldsymbol{u}^{*}, \delta^{*}, \omega^{*}\right)$ such that the associated displacement field $v^{*}$ given by (50) is admissible. These admissibility conditions will be specified in the next subsection.

Proposition 3. The macroscopic displacement fields $(\boldsymbol{u}, \delta, \omega)$ are a stationary point of the following potential energy $\mathscr{P}^{0}$ :

$$
\begin{aligned}
\mathscr{P}^{0}\left(\boldsymbol{u}^{*}, \delta^{*}, \omega^{*}\right) \\
=\int_{\Omega_{c}} \frac{1}{2} A^{c} \boldsymbol{\varepsilon}\left(\boldsymbol{u}^{*}\right) \cdot \boldsymbol{\varepsilon}\left(\boldsymbol{u}^{*}\right) d x+\int_{\Omega_{d}} \frac{T}{2} \frac{\partial \omega^{*}}{\partial x_{3}} \frac{\partial \omega^{*}}{\partial x_{3}} d x \\
\quad+\int_{\Omega_{d}}\left(\frac{1}{2} \mathrm{~A}^{d} \boldsymbol{\varepsilon}\left(\boldsymbol{u}^{*}\right) \cdot \boldsymbol{\varepsilon}\left(\boldsymbol{u}^{*}\right)+\boldsymbol{\Sigma} \cdot \boldsymbol{\varepsilon}\left(\boldsymbol{u}^{*}\right) \frac{\partial \delta^{*}}{\partial x_{3}}+\frac{K}{2} \frac{\partial \delta^{*}}{\partial x_{3}} \frac{\partial \delta^{*}}{\partial x_{3}}\right) d x \\
\quad-\int_{\Omega}\langle\rho\rangle \boldsymbol{g} \cdot \boldsymbol{u}^{*} d x-\int_{\Omega_{d}} \rho_{f} V_{f} g_{3} \delta^{*} d x-\int_{\Gamma_{s}}\left(\boldsymbol{F} \cdot \boldsymbol{u}^{*}+V_{f} F_{3} \delta^{*}\right) d \Gamma,
\end{aligned}
$$


where the effective stiffness tensors $\mathrm{A}^{c}$ and $\mathrm{A}^{d}$, the effective stress tensor $\boldsymbol{\Sigma}$ and the effective rigidity coefficients $K$ and $T$ are obtained by solving the different cell problems. Specifically, $\mathrm{A}^{c}$ is given by (56), $\mathrm{A}^{d}$ by (58), $\Sigma=\left\langle S^{3}\right\rangle$ and $K=\left\langle S_{33}^{3}\right\rangle_{f}$, where $S^{3}$ is given by (47)-(48) and $T=\pi R^{4} \mu_{f} /(2|\boldsymbol{V}|)$.

Proof. It suffices to remark that (63) is equivalent to

$$
\left.\frac{d}{d h} \mathscr{P}^{0}\left(\boldsymbol{u}+h \boldsymbol{u}^{*}, \delta+h \delta^{*}, \omega+h \omega^{*}\right)\right|_{h=0}=0 .
$$

Hence, $\mathscr{P}^{0}$ can be seen as the effective potential energy of the composite body.

\section{Discussion and examples}

\subsection{Properties of the effective coefficients.}

Proposition 4. The effective rigidity tensor $\mathrm{A}^{c}$ of the perfectly bonded composite satisfies the minimization problem

$$
\text { for } \boldsymbol{\varepsilon}^{*} \in \mathbb{M}_{s}^{3}, \quad \mathrm{~A}^{c} \boldsymbol{\varepsilon}^{*} \cdot \boldsymbol{\varepsilon}^{*}=\min _{\boldsymbol{w} \in \mathscr{H}_{c}} \mathscr{E}^{c}(\boldsymbol{w}),
$$

where

$$
\mathscr{E}^{c}(\boldsymbol{w})=\left\langle\mathrm{A}\left(\boldsymbol{\varepsilon}^{*}+\boldsymbol{\varepsilon}(\boldsymbol{w})\right) \cdot\left(\boldsymbol{\varepsilon}^{*}+\boldsymbol{\varepsilon}(\boldsymbol{w})\right)\right\rangle .
$$

The effective rigidity tensor $\mathrm{A}^{d}$, the effective tensor $\boldsymbol{\Sigma}$ and the effective rigidity coefficient $K$ of the debonded composite satisfy the minimization problem

$$
\text { for } \boldsymbol{\varepsilon}^{*} \in \mathbb{M}_{s}^{3} \text { and } d^{*} \in \mathbb{R}, \quad \mathrm{A}^{d} \boldsymbol{\varepsilon}^{*} \cdot \boldsymbol{\varepsilon}^{*}+2 d^{*} \boldsymbol{\Sigma} \cdot \boldsymbol{\varepsilon}^{*}+K d^{* 2}=\min _{\boldsymbol{w} \in \mathscr{H}_{d}} \mathscr{E}^{d}(\boldsymbol{w}),
$$

where

$$
\begin{aligned}
\mathscr{E}^{d}(\boldsymbol{w})=\left\langle\mathrm{A}^{m}\left(\boldsymbol{\varepsilon}^{*}+\boldsymbol{\varepsilon}(\boldsymbol{w})\right) \cdot\right. & \left.\left(\boldsymbol{\varepsilon}^{*}+\boldsymbol{\varepsilon}(\boldsymbol{w})\right)\right\rangle_{m} \\
& +\left\langle\mathrm{A}^{f}\left(\boldsymbol{\varepsilon}^{*}+d^{*} \boldsymbol{e}_{3} \otimes \boldsymbol{e}_{3}+\boldsymbol{\varepsilon}(\boldsymbol{w})\right) \cdot\left(\boldsymbol{\varepsilon}^{*}+d^{*} \boldsymbol{e}_{3} \otimes \boldsymbol{e}_{3}+\boldsymbol{\varepsilon}(\boldsymbol{w})\right)\right\rangle_{f} .
\end{aligned}
$$

Therefore, there exist two positive constants $\alpha_{c}>0$ and $\alpha_{d}>0$ such that, for all $\boldsymbol{\varepsilon}^{*} \in \mathbb{M}_{s}^{3}$ and all $d^{*} \in \mathbb{R}$,

$$
\mathrm{A}^{c} \boldsymbol{\varepsilon}^{*} \cdot \boldsymbol{\varepsilon}^{*} \geq \alpha_{c} \boldsymbol{\varepsilon}^{*} \cdot \boldsymbol{\varepsilon}^{*}, \quad \mathrm{~A}^{d} \boldsymbol{\varepsilon}^{*} \cdot \boldsymbol{\varepsilon}^{*}+2 d^{*} \boldsymbol{\Sigma} \cdot \boldsymbol{\varepsilon}^{*}+K d^{* 2} \geq \alpha_{d}\left(\boldsymbol{\varepsilon}^{*} \cdot \boldsymbol{\varepsilon}^{*}+d^{* 2}\right) .
$$

Moreover, $\mathrm{A}^{c}$ and $\mathrm{A}^{d}$ are well ordered in the sense that

$$
\mathrm{A}^{c} \boldsymbol{\varepsilon}^{*} \cdot \boldsymbol{\varepsilon}^{*} \geq \mathrm{A}^{d} \boldsymbol{\varepsilon}^{*} \cdot \boldsymbol{\varepsilon}^{*} \text { for all } \boldsymbol{\varepsilon}^{*} \in \mathbb{M}_{s}^{3} .
$$

Proof. Let us prove the property of minimization for the debonded composite, the proof being similar for the perfectly bonded composite. Let $w^{*}$ be a minimizer 
of $\mathscr{E}^{d}$ over $\mathscr{H}_{d} ; \boldsymbol{w}^{*}$ is unique up to an element of $\mathscr{R}_{d}$ and satisfies the variational equation

$$
\left\langle\mathrm{A}^{m}\left(\boldsymbol{\varepsilon}^{*}+\boldsymbol{\varepsilon}\left(\boldsymbol{w}^{*}\right)\right) \cdot \boldsymbol{\varepsilon}(\boldsymbol{w})\right\rangle_{m}+\left\langle\mathrm{A}^{f}\left(\boldsymbol{\varepsilon}^{*}+d^{*} \boldsymbol{e}_{3} \otimes \boldsymbol{e}_{3}+\boldsymbol{\varepsilon}\left(\boldsymbol{w}^{*}\right)\right) \cdot \boldsymbol{\varepsilon}(\boldsymbol{w})\right\rangle_{f}=0
$$

for all $w \in \mathscr{H}_{d}$.

By linearity and using (34)-(35), one deduces that $\boldsymbol{w}^{*}(\boldsymbol{y})=\varepsilon_{i j}^{*} \xi^{i j}(\boldsymbol{y})+d^{*} \boldsymbol{D}^{3}(\boldsymbol{y})$. Moreover, using (68) with $\boldsymbol{w}=\boldsymbol{w}^{*}$ yields

$$
\begin{aligned}
\mathscr{E}^{d}\left(\boldsymbol{w}^{*}\right)=\left\langle\mathrm{A}^{m} \boldsymbol{\varepsilon}^{*} \cdot \boldsymbol{\varepsilon}^{*}-\mathrm{A}^{m} \boldsymbol{\varepsilon}\left(\boldsymbol{w}^{*}\right) \cdot \boldsymbol{\varepsilon}\left(\boldsymbol{w}^{*}\right)\right\rangle_{m} \\
\quad+\left\langle\mathrm{A}^{f}\left(\boldsymbol{\varepsilon}^{*}+d^{*} \boldsymbol{e}_{3} \otimes \boldsymbol{e}_{3}\right) \cdot\left(\boldsymbol{\varepsilon}^{*}+d^{*} \boldsymbol{e}_{3} \otimes \boldsymbol{e}_{3}\right)-\mathrm{A}^{f} \boldsymbol{\varepsilon}\left(\boldsymbol{w}^{*}\right) \cdot \boldsymbol{\varepsilon}\left(\boldsymbol{w}^{*}\right)\right\rangle_{f} \\
=\left\langle\mathrm{A} \boldsymbol{\varepsilon}^{*} \cdot \boldsymbol{\varepsilon}^{*}-\mathrm{A} \boldsymbol{\varepsilon}\left(\boldsymbol{w}^{*}\right) \cdot \boldsymbol{\varepsilon}\left(\boldsymbol{w}^{*}\right)\right\rangle+2 V_{f} \mathrm{~A}_{33 i j}^{f} \varepsilon_{i j}^{*} d^{*}+V_{f} \mathrm{~A}_{3333}^{f} d^{* 2} \\
=\left\langle\mathrm{A}_{i j k l}-\mathrm{A} \boldsymbol{\varepsilon}\left(\boldsymbol{\xi}^{i j}\right) \cdot \boldsymbol{\varepsilon}\left(\boldsymbol{\xi}^{k l}\right)\right\rangle \varepsilon_{i j}^{*} \varepsilon_{k l}^{*}+2\left(V_{f} \mathrm{~A}_{33 i j}^{f}-\left\langle\mathrm{A} \boldsymbol{\varepsilon}\left(\boldsymbol{\xi}^{i j}\right) \cdot \boldsymbol{\varepsilon}\left(\boldsymbol{D}^{3}\right)\right\rangle\right) \varepsilon_{i j}^{*} d^{*} \\
\quad+\left(V_{f} \mathrm{~A}_{3333}^{f}-\left\langle\mathrm{A} \boldsymbol{\varepsilon}\left(\boldsymbol{D}^{3}\right) \cdot \boldsymbol{\varepsilon}\left(\boldsymbol{D}^{3}\right)\right\rangle\right) d^{* 2} .
\end{aligned}
$$

Using (34) with $\boldsymbol{w}=\boldsymbol{D}^{3}$, (35) with $\boldsymbol{D}^{i}=\boldsymbol{w}=\boldsymbol{D}^{3}$ and (58), one gets $\mathscr{E}^{d}\left(\boldsymbol{w}^{*}\right)=\mathrm{A}^{d} \boldsymbol{\varepsilon}^{*} \cdot \boldsymbol{\varepsilon}^{*}+2\left(V_{f} \mathrm{~A}_{33 i j}^{f}+\left\langle\mathrm{A}_{i j k l} \varepsilon\left(\boldsymbol{D}^{3}\right)_{k l}\right\rangle\right) \varepsilon_{i j}^{*} d^{*}+\left\langle\mathrm{A}_{3333}^{f}+\mathrm{A}_{33 k l}^{f} \varepsilon\left(\boldsymbol{D}^{3}\right)_{k l}\right\rangle_{f} d^{* 2}$.

Then it suffices to use (44) with $i=3$ to obtain that $V_{f} \mathrm{~A}_{33 i j}^{f}+\left\langle\mathrm{A}_{i j k l} \varepsilon\left(D^{3}\right)_{k l}\right\rangle=$ $\left\langle S_{i j}^{3}\right\rangle=\Sigma_{i j}$ and $\left\langle\mathrm{A}_{3333}^{f}+\mathrm{A}_{33 k l}^{f} \varepsilon\left(\boldsymbol{D}^{3}\right)_{k l}\right\rangle_{f}=\left\langle S_{33}^{3}\right\rangle_{f}=K$. This yields (66).

We now prove the positivity of $\mathscr{E} d\left(\boldsymbol{w}^{*}\right)$. First, $\mathscr{C}^{d}\left(\boldsymbol{w}^{*}\right) \geq 0$ by definition and by the positivity of $\mathrm{A}^{m}$ and $\mathrm{A}^{f}$. We show that equality holds if and only if $\boldsymbol{\varepsilon}^{*}=\mathbf{0}$ and $d^{*}=0$. By the expression of $\mathscr{E} d\left(\boldsymbol{w}^{*}\right)$, equality holds if and only if

$$
\boldsymbol{\varepsilon}\left(\boldsymbol{w}^{*}\right)(\boldsymbol{y})=\left\{\begin{array}{cl}
-\boldsymbol{\varepsilon}^{*} & \text { for all } \boldsymbol{y} \in \boldsymbol{V}_{m}, \\
-\boldsymbol{\varepsilon}^{*}-d^{*} \boldsymbol{e}_{3} \otimes \boldsymbol{e}_{3} & \text { for all } \boldsymbol{y} \in \boldsymbol{V}_{f} .
\end{array}\right.
$$

But since $\varepsilon\left(\boldsymbol{w}^{*}\right)_{33}=0$, one gets $\varepsilon_{33}^{*}=d^{*}=0$. Accordingly, $\boldsymbol{\varepsilon}\left(\boldsymbol{w}^{*}\right)(\boldsymbol{y})=-\boldsymbol{\varepsilon}^{*}$ for all $\boldsymbol{y} \in \boldsymbol{V} \backslash \boldsymbol{I}$. But, since $\boldsymbol{w}^{*}$ is $\boldsymbol{V}$-periodic, one finally gets $\boldsymbol{\varepsilon}^{*}=\mathbf{0}$. Therefore the quadratic form $\mathrm{A}^{d} \boldsymbol{\varepsilon}^{*} \cdot \boldsymbol{\varepsilon}^{*}+2 d^{*} \boldsymbol{\Sigma} \cdot \boldsymbol{\varepsilon}^{*}+K d^{* 2}$ is definite positive on $\mathbb{M}_{s}^{3} \times \mathbb{R}$.

To prove that $\mathrm{A}^{c}$ and $\mathrm{A}^{d}$ are well ordered, let us take $d^{*}=0$. Then, by virtue of the minimization properties, one gets

$$
\begin{aligned}
& \mathrm{A}^{c} \boldsymbol{\varepsilon}^{*} \cdot \boldsymbol{\varepsilon}^{*}=\min _{\boldsymbol{w} \in \mathscr{H}_{c}}\left\langle\mathrm{~A}\left(\boldsymbol{\varepsilon}^{*}+\boldsymbol{\varepsilon}(\boldsymbol{w})\right) \cdot\left(\boldsymbol{\varepsilon}^{*}+\boldsymbol{\varepsilon}(\boldsymbol{w})\right)\right\rangle, \\
& \mathrm{A}^{d} \boldsymbol{\varepsilon}^{*} \cdot \boldsymbol{\varepsilon}^{*}=\min _{\boldsymbol{w} \in \mathscr{H}_{d}}\left\langle\mathrm{~A}\left(\boldsymbol{\varepsilon}^{*}+\boldsymbol{\varepsilon}(\boldsymbol{w})\right) \cdot\left(\boldsymbol{\varepsilon}^{*}+\boldsymbol{\varepsilon}(\boldsymbol{w})\right)\right\rangle .
\end{aligned}
$$

Since $\mathscr{H}_{c} \subset \mathscr{H}_{d}$, one obtains the desired inequality $\mathrm{A}^{c} \boldsymbol{\varepsilon}^{*} \cdot \boldsymbol{\varepsilon}^{*} \geq \mathrm{A}^{d} \boldsymbol{\varepsilon}^{*} \cdot \boldsymbol{\varepsilon}^{*}$ for all $\boldsymbol{\varepsilon}^{*}$ in $\mathbb{M}_{s}^{3}$.

4.2. The relevant functional framework of the effective model. Let us discuss here what are the relevant functional spaces so that the effective problem coming from the asymptotic analysis is well posed. The natural framework is the set of 
all functions with finite energy $\mathscr{P}^{0}$. Specifically, $\boldsymbol{u}^{*}$ must belong to $H^{1}\left(\Omega, \mathbb{R}^{3}\right)$ while $\delta^{*}$ and $\omega^{*}$ must belong to $H_{L}^{1}\left(\Omega_{d}\right)$, where

$$
H_{L}^{1}\left(\Omega_{d}\right)=\left\{\varphi: \varphi=0 \text { in } \Omega_{c}, \varphi \in L^{2}\left(\Omega_{d}\right), \frac{\partial \varphi}{\partial x_{3}} \in L^{2}\left(\Omega_{d}\right)\right\} .
$$

Accordingly, one can define as usual the trace of $\boldsymbol{u}^{*}$ on the boundary of $\Omega$ (and more generally on any sufficiently smooth surface included in $\bar{\Omega}$ ). Therefore, the Dirichlet boundary condition $\boldsymbol{u}^{*}=\mathbf{0}$ on $\Gamma_{c}$ has a sense. But this is not the case for the elements of $H_{L}^{1}\left(\Omega_{d}\right)$. Indeed, since one only controls its first derivative with respect to $x_{3}$, one can define the trace of such an element $\varphi$ on surfaces of the type $x_{3}=$ constant but not necessarily on surfaces with arbitrary orientations. Accordingly, the definition of the boundary conditions on $\Gamma_{c}$ and the continuity conditions at the interface between $\Omega_{c}$ and $\Omega_{d}$ need more developed arguments which are outside the scope of the present paper. As far as the linear part of the potential energy is concerned, the work done by the external forces is finite provided that the density $\boldsymbol{g}$ and $\boldsymbol{F}$ are sufficiently smooth. For the work of the specific forces, it suffices that $\boldsymbol{g}$ be in $L^{2}\left(\Omega ; \mathbb{R}^{3}\right)$ in order that both integrals over $\Omega$ and $\Omega_{d}$ be finite. The question is more delicate for $\boldsymbol{F}$. It is sufficient that $\boldsymbol{F}$ be in $L^{2}\left(\Gamma_{s} ; \mathbb{R}^{3}\right)$ in order that $\int_{\Gamma_{s}} \boldsymbol{F} \cdot \boldsymbol{u}^{*} d \Gamma<+\infty$. But, the term $\int_{\Gamma_{s} \cap{ }_{2} \Omega_{d}} F_{3} \delta^{*} d \Gamma$ makes sense only on the part of the boundary where either $F_{3}=0$ or $\delta^{*}$ is defined. Accordingly, we will assume that the following hypothesis holds:

Hypothesis 1. The given density of forces is such that $g \in L^{2}\left(\Omega ; \mathbb{R}^{3}\right)$ and $\boldsymbol{F} \in$ $L^{2}\left(\Gamma_{s} ; \mathbb{R}^{3}\right)$. Moreover, on the part $\Gamma_{s} \cap \partial \Omega_{d}, F_{3}=0$.

Finally, introducing the set of all kinematically admissible displacement fields

$$
\mathscr{C}^{0}=\left\{\left(\boldsymbol{u}^{*}, \delta^{*}, \omega^{*}\right) \in H^{1}\left(\Omega ; \mathbb{R}^{3}\right) \times H_{L}^{1}\left(\Omega_{d}\right)^{2}: \boldsymbol{u}^{*}=0 \text { on } \Gamma_{c}\right\},
$$

the effective problem can be formulated as follows:

$$
\text { find }(\boldsymbol{u}, \delta, \omega) \in \mathscr{C}^{0} \text { which minimizes } \mathscr{P}^{0} \text { over } \mathscr{C}^{0} \text {. }
$$

We are now in the position to establish the final result.

Proposition 5. Let $\mathscr{R}^{0}$ be the subset of $\mathscr{C}^{0}$ made of all displacement fields with null elastic energy:

$$
\mathscr{R}^{0}=\left\{\left(\boldsymbol{u}^{*}, \delta^{*}, \omega^{*}\right) \in \mathscr{C}^{0}: \boldsymbol{\varepsilon}\left(\boldsymbol{u}^{*}\right)=\mathbf{0} \text { in } \Omega, \frac{\partial \delta^{*}}{\partial x_{3}}=\frac{\partial \omega^{*}}{\partial x_{3}}=0 \text { in } \Omega_{d}\right\} .
$$

Then, if $\mathscr{R}^{0}=\{(\mathbf{0}, 0,0)\}$ and if the given forces $\boldsymbol{g}$ and $\boldsymbol{F}$ satisfy Hypothesis 1 , problem (70) admits a unique solution. 
Proof. Uniqueness is guaranteed by virtue of the assumption on $\mathscr{R}^{0}$ and of the positivity of the elastic energy. The existence is due to the smoothness assumption on the loading and to the positivity property (67) which ensures the coercivity.

Remark 4. The relative rotation of the fiber $\omega^{*}$ is not coupled with the macroscopic displacement field $\boldsymbol{u}^{*}$ and the sliding of the fiber $\delta^{*}$ in the elastic energy. Since $\omega^{*}$ does not appear in the work of the given external forces, one immediately obtains that the solution is such that $\partial \omega / \partial x_{3}=0$ in $\Omega_{d}$ and hence there does not exist a fiber torsional energy. But this property will no longer hold true if one changes some assumptions on the composite behavior or on the loading.

The solution $(\boldsymbol{u}, \delta)$ of the effective problem satisfies the following set of local equilibrium equations in $\Omega_{d}$ :

$$
\left\{\begin{array}{r}
\operatorname{div}\left(\mathrm{A}^{d} \boldsymbol{\varepsilon}(\boldsymbol{u})+\frac{\partial \delta}{\partial x_{3}} \boldsymbol{\Sigma}\right)+\langle\rho\rangle \boldsymbol{g}=\mathbf{0}, \\
\frac{\partial}{\partial x_{3}}\left(K \frac{\partial \delta}{\partial x_{3}}+\boldsymbol{\Sigma} \cdot \boldsymbol{\varepsilon}(\boldsymbol{u})\right)+V_{f} \rho_{f} g_{3}=0 .
\end{array}\right.
$$

These equations must be understood in the sense of distributions when the loading is not sufficiently smooth. The first one is a vectorial equation while the second one is scalar. Both are second-order partial differential equations and they are coupled by the term which involves the effective internal stress tensor $\boldsymbol{\Sigma}$.

4.3. Case of a regular hexagonal cell. Let $L$ be a characteristic length of the body, $\ell=3^{-1 / 4} \sqrt{2} L, \boldsymbol{a}=\ell \boldsymbol{e}_{1}, \boldsymbol{b}=\ell\left(\boldsymbol{e}_{1}+\sqrt{3} \boldsymbol{e}_{2}\right) / 2$ and $\boldsymbol{V}_{f}$ be the disk of center 0 and radius $R<\ell / 2$. Thus $\boldsymbol{V}$ is a regular hexagon centered at $\mathbf{0}$ with area $L^{2}$; see Figure 2. Since the material is isotropic, we can use the results of [Léné 1984] to obtain that $\mathrm{A}^{c}$ and $\mathrm{A}^{d}$ are positive transversely isotropic fourth-order tensors with axis $\boldsymbol{e}_{3}$. Therefore, $\mathrm{A}^{c}$ and $\mathrm{A}^{d}$ are such that, for all $\boldsymbol{\varepsilon} \in \mathbb{M}_{s}^{3}$,

$$
\begin{aligned}
& \mathrm{A}^{c} \boldsymbol{\varepsilon} \cdot \boldsymbol{\varepsilon}=A_{L}^{c} \varepsilon_{33}^{2}+\lambda_{L}^{c} \varepsilon_{33} \varepsilon_{\alpha \alpha}+\lambda_{T}^{c} \varepsilon_{\alpha \alpha}^{2}+2 \mu_{T}^{c} \varepsilon_{\alpha \beta} \varepsilon_{\alpha \beta}+2 \mu_{L}^{c} \varepsilon_{3 \alpha} \varepsilon_{3 \alpha}, \\
& \mathrm{A}^{d} \boldsymbol{\varepsilon} \cdot \boldsymbol{\varepsilon}=A_{L}^{d} \varepsilon_{33}^{2}+\lambda_{L}^{d} \varepsilon_{33} \varepsilon_{\alpha \alpha}+\lambda_{T}^{d} \varepsilon_{\alpha \alpha}^{2}+2 \mu_{T}^{d} \varepsilon_{\alpha \beta} \varepsilon_{\alpha \beta}+2 \mu_{L}^{d} \varepsilon_{3 \alpha} \varepsilon_{3 \alpha},
\end{aligned}
$$
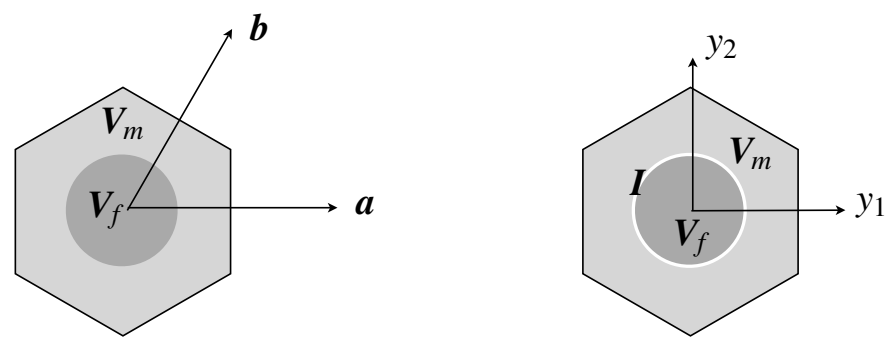

Figure 2. The case when the cell is a regular hexagon (left: bonded; right: debonded). 
where the ten moduli satisfy the following inequalities:

$$
\begin{gathered}
A_{L}^{c} \geq A_{L}^{d}>0, \quad \mu_{T}^{c} \geq \mu_{T}^{d}>0, \quad \mu_{L}^{c} \geq \mu_{L}^{d}>0, \\
A_{L}^{c}\left(\lambda_{T}^{c}+\mu_{T}^{c}\right)>\lambda_{L}^{c 2}, \quad A_{L}^{d}\left(\lambda_{T}^{d}+\mu_{T}^{d}\right)>\lambda_{L}^{d^{2}} .
\end{gathered}
$$

In the same manner, $\Sigma$ is transversely isotropic and hence can read

$$
\boldsymbol{\Sigma}=\sigma_{T}\left(\boldsymbol{e}_{1} \otimes \boldsymbol{e}_{1}+\boldsymbol{e}_{2} \otimes \boldsymbol{e}_{2}\right)+\sigma_{L} \boldsymbol{e}_{3} \otimes \boldsymbol{e}_{3}
$$

Let us compare the longitudinal shear moduli $\mu_{L}^{c}$ and $\mu_{L}^{d}$. They are given, respectively, by the two antiplane minimization cell problems

$$
\begin{aligned}
& \mu_{L}^{c}=\min _{\varphi \in H_{\#}^{1}(\boldsymbol{V})}\left\langle\mu\left(\nabla \varphi+\boldsymbol{e}_{1}\right) \cdot\left(\nabla \varphi+\boldsymbol{e}_{1}\right)\right\rangle, \\
& \mu_{L}^{d}=\min _{\varphi \in H_{\#}^{1}(\boldsymbol{V} \backslash \boldsymbol{I})}\left\langle\mu\left(\nabla \varphi+\boldsymbol{e}_{1}\right) \cdot\left(\nabla \varphi+\boldsymbol{e}_{1}\right)\right\rangle .
\end{aligned}
$$

The minimizers are the nonzero components $\chi_{3}^{13}$ and $\xi_{3}^{13}$ of $\chi^{13}$ and $\xi^{13}$. They satisfy

$$
\begin{array}{ll}
0=\left\langle\mu\left(\nabla \chi_{3}^{13}+\boldsymbol{e}_{1}\right) \cdot \nabla \varphi\right\rangle & \text { for all } \varphi \in H_{\#}^{1}(\boldsymbol{V}), \\
0=\left\langle\mu\left(\nabla \xi_{3}^{13}+\boldsymbol{e}_{1}\right) \cdot \nabla \varphi\right\rangle & \text { for all } \varphi \in H_{\#}^{1}(\boldsymbol{V} \backslash \boldsymbol{I}),
\end{array}
$$

where \# stands for periodic. It is easy to check that $\xi_{3}^{13}(\boldsymbol{y})=-y_{1}$ (plus an arbitrary constant) in $\boldsymbol{V}_{f}$. Therefore

$$
\mu_{L}^{d}=\left\langle\mu_{m}\left(\nabla \xi_{3}^{13}+\boldsymbol{e}_{1}\right) \cdot\left(\nabla \xi_{3}^{13}+\boldsymbol{e}_{1}\right)\right\rangle_{m}=\min _{\varphi \in H_{\#}^{1}\left(\boldsymbol{V}_{m}\right)}\left\langle\mu_{m}\left(\nabla \varphi+\boldsymbol{e}_{1}\right) \cdot\left(\nabla \varphi+\boldsymbol{e}_{1}\right)\right\rangle_{m} .
$$

In other words, the longitudinal shear modulus of the debonded composite is as if there were a hole instead of a fiber. Accordingly, $\mu_{L}^{c}$ and $\mu_{L}^{d}$ satisfy the following bounds:

$$
0<\mu_{L}^{d}<V_{m} \mu_{m}<\frac{1}{\frac{V_{m}}{\mu_{m}}+\frac{V_{f}}{\mu_{f}}}<\mu_{L}^{c}<V_{m} \mu_{m}+V_{f} \mu_{f},
$$

the last two inequalities corresponding to the classical Voigt and Reuss bounds.

In the particular case where the Poisson ratios of the fibers and the matrix equal 0 , then $\lambda_{f}=\lambda_{m}=0$. Moreover $\mu_{f}=E_{f}$ and $\mu_{m}=E_{m}, E_{f}$ and $E_{m}$ denoting the Young moduli of the fibers and the matrix. In this case, one easily deduces from (32), (34) and (35) that

$$
\chi^{33}=\xi^{33}=D^{3}=\mathbf{0}
$$

Therefore, one gets

$$
A_{L}^{c}=A_{L}^{d}=V_{m} E_{m}+V_{f} E_{f}, \quad \lambda_{L}^{c}=\lambda_{L}^{d}=0, \quad \sigma_{T}=0, \quad \sigma_{L}=K=V_{f} E_{f} .
$$

Let us remark that $\mathrm{A}^{c}$ and $\mathrm{A}^{d}$ are not strictly well ordered because $A_{L}^{c}=A_{L}^{d}$. 
4.4. Example. Let us finish this section by an example of application. We consider a cylinder $\Omega=S \times(0, L)$ whose cross-section $S$ is an open connected bounded subset of $\mathbb{R}^{2}$ and whose axis $\boldsymbol{e}_{3}$ corresponds to the vertical. This cylinder, submitted to the uniform gravity $\boldsymbol{g}=-g \boldsymbol{e}_{3}$, is fixed on its section $S \times\{L\}$ and free on all other boundaries $S \times\{0\}$ and $\partial S \times(0, L)$. It is made of a unidirectional composite, the fibers of which are periodically distributed according to a regular hexagonal lattice with axis $\boldsymbol{e}_{3}$. The Poisson ratios of the fibers and the matrix are equal to 0 . Accordingly, we are in the situation described at the end of the previous subsection; i.e.,

$$
\begin{aligned}
& \mathrm{A}^{c} \boldsymbol{\varepsilon} \cdot \boldsymbol{\varepsilon}=\langle E\rangle \varepsilon_{33}^{2}+\lambda_{T}^{c} \varepsilon_{\alpha \alpha}^{2}+2 \mu_{T}^{c} \varepsilon_{\alpha \beta} \varepsilon_{\alpha \beta}+2 \mu_{L}^{c} \varepsilon_{3 \alpha} \varepsilon_{3 \alpha}, \\
& \mathrm{A}^{d} \boldsymbol{\varepsilon} \cdot \boldsymbol{\varepsilon}=\langle E\rangle \varepsilon_{33}^{2}+\lambda_{T}^{d} \varepsilon_{\alpha \alpha}^{2}+2 \mu_{T}^{d} \varepsilon_{\alpha \beta} \varepsilon_{\alpha \beta}+2 \mu_{L}^{d} \varepsilon_{3 \alpha} \varepsilon_{3 \alpha}, \\
& \\
& \boldsymbol{\Sigma}=E_{f} V_{f} \boldsymbol{e}_{3} \otimes \boldsymbol{e}_{3}, \quad K=E_{f} V_{f} .
\end{aligned}
$$

Moreover, we assume that the fibers are debonded in the part $\Omega_{d}=S \times(0, \ell)$ and still bonded in the complementary part $\Omega_{c}=S \times(\ell, L)$ where $0<\ell<L$. Accordingly, the work of the gravity reads as

$$
f^{0}\left(\boldsymbol{u}^{*}, \delta^{*}\right)=-\int_{S \times(0, L)}\langle\rho\rangle g u_{3}^{*} d x-\int_{S \times(0, \ell)} \rho_{f} V_{f} g \delta^{*} d x,
$$

and the conditions of admissibility for the displacement fields are

$$
\begin{gathered}
\boldsymbol{u}^{*} \in H^{1}\left(S \times(0, L) ; \mathbb{R}^{3}\right), \quad\left(\delta^{*}, \omega^{*}\right) \in H_{L}^{1}(S \times(0, \ell))^{2}, \\
\boldsymbol{u}^{*}=\mathbf{0} \text { on } S \times\{L\}, \quad \delta^{*}=\omega^{*}=0 \text { on } S \times\{\ell\} .
\end{gathered}
$$

Therefore $\mathscr{R}^{0}=(\mathbf{0}, 0,0)$, we are in the situation of Proposition 5 and the effective problem admits a unique solution. Let us search for the solution under the form

$$
\boldsymbol{u}(\boldsymbol{x})=u\left(x_{3}\right) \boldsymbol{e}_{3}, \quad \delta(\boldsymbol{x})=\delta\left(\boldsymbol{x}_{3}\right), \quad \omega(\boldsymbol{x})=0 \quad \text { with } u(L)=0, \quad \delta(\ell)=0 .
$$

Then, the effective stress reads as

$$
\mathrm{A}^{c} \boldsymbol{\varepsilon}(\boldsymbol{u})(\boldsymbol{x})=\mathrm{A}^{d} \boldsymbol{\varepsilon}(\boldsymbol{u})(\boldsymbol{x})=\langle E\rangle u^{\prime}\left(x_{3}\right) \boldsymbol{e}_{3} \otimes \boldsymbol{e}_{3},
$$

where the prime denotes the derivative with respect to $x_{3}$. Inserting this form into (63), the variational effective problem becomes

$$
\begin{gathered}
0=\int_{S \times(0, \ell)}\left(\left(\langle E\rangle u^{\prime}+E_{f} V_{f} \delta^{\prime}\right) \frac{\partial u_{3}^{*}}{\partial x_{3}}+\langle\rho\rangle g u_{3}^{*}+E_{f} V_{f}\left(\delta^{\prime}+u^{\prime}\right) \frac{\partial \delta^{*}}{\partial x_{3}}+\rho_{f} V_{f} g \delta^{*}\right) d x \\
\quad+\int_{S \times(\ell, L)}\left(\langle E\rangle u^{\prime} \frac{\partial u_{3}^{*}}{\partial x_{3}}+\langle\rho\rangle g u_{3}^{*}\right) d x,
\end{gathered}
$$

and the equality must hold for all admissible $\left(\boldsymbol{u}^{*}, \delta^{*}\right)$. Taking first $\left(\boldsymbol{u}^{*}, \delta^{*}\right)$ of the same form as the expected solution, i.e., $\boldsymbol{u}^{*}(\boldsymbol{x})=v\left(x_{3}\right) \boldsymbol{e}_{3}$ and $\delta^{*}(\boldsymbol{x})=\varphi\left(x_{3}\right)$, we 
obtain the following one-dimensional variational problem for $(u, \delta)$ :

$$
\begin{aligned}
0=\int_{0}^{\ell}\left(\left(\langle E\rangle u^{\prime}+E_{f} V_{f} \delta^{\prime}\right) v^{\prime}+\langle\rho\rangle g v+E_{f} V_{f}\left(\delta^{\prime}+u^{\prime}\right) \varphi^{\prime}\right. & \left.+\rho_{f} V_{f} g \varphi\right) d x_{3} \\
& +\int_{\ell}^{L}\left(\langle E\rangle u^{\prime} v^{\prime}+\langle\rho\rangle g v\right) d x_{3},
\end{aligned}
$$

where the equality must hold for all $v \in H^{1}(0, L)$ such that $v(L)=0$ and all $\varphi \in$ $H^{1}(0, \ell)$ such that $\varphi(\ell)=0$. By standard arguments of calculus of variations, we find that $u$ and $\delta$ are the unique solution of the following boundary value problem:

$$
\begin{aligned}
& \text { in }(0, \ell): \quad\left\{\begin{array}{l}
\langle E\rangle u^{\prime \prime}+E_{f} V_{f} \delta^{\prime \prime}=\langle\rho\rangle g, \\
E_{f}\left(\delta^{\prime \prime}+u^{\prime \prime}\right)=\rho_{f} g ;
\end{array} \quad \text { in }(\ell, L): \quad\langle E\rangle u^{\prime \prime}=\langle\rho\rangle g ;\right. \\
& u^{\prime}(0)=\delta^{\prime}(0)=0 ; \quad \delta(\ell)=0, \quad \llbracket u \rrbracket(\ell)=0, \\
& \langle E\rangle \llbracket u^{\prime} \rrbracket(\ell)=E_{f} V_{f} \delta^{\prime}(\ell-) ; \quad u(L)=0 .
\end{aligned}
$$

After some calculations, we eventually find

$$
u^{\prime}\left(\boldsymbol{x}_{3}\right)=\left\{\begin{array}{ll}
\frac{\rho_{m}}{E_{m}} g x_{3}, & 0<x_{3}<\ell, \\
\frac{\langle\rho\rangle}{\langle E\rangle} g x_{3}, & \ell<x_{3}<L,
\end{array} \quad u(L)=0, \quad \delta\left(x_{3}\right)=\left(\frac{\rho_{f}}{E_{f}}-\frac{\rho_{m}}{E_{m}}\right) \frac{g}{2}\left(x_{3}^{2}-\ell^{2}\right) .\right.
$$

Conversely, the reader could verify that (77) is satisfied for any admissible $\left(\boldsymbol{u}^{*}, \delta^{*}\right)$ with $(u, \delta)$ given by $(80)$. Therefore, we have found the unique solution of the effective problem. Using (42) and (49), we can see the influence of the debonding on the repartition of the stresses inside the composite:

$$
\begin{aligned}
& \text { in } S \times(0, \ell): \quad \boldsymbol{\sigma}^{0}(\boldsymbol{x}, \boldsymbol{y})= \begin{cases}\frac{E_{f}}{\langle E\rangle}\langle\rho\rangle g x_{3} \boldsymbol{e}_{3} \otimes \boldsymbol{e}_{3} & \text { in } \boldsymbol{V}_{f}, \\
\frac{E_{m}}{\langle E\rangle}\langle\rho\rangle g x_{3} \boldsymbol{e}_{3} \otimes \boldsymbol{e}_{3} & \text { in } \boldsymbol{V}_{m},\end{cases} \\
& \text { in } S \times(\ell, L): \quad \boldsymbol{\sigma}^{0}(\boldsymbol{x}, \boldsymbol{y})= \begin{cases}\rho_{f} g x_{3} \boldsymbol{e}_{3} \otimes \boldsymbol{e}_{3} & \text { in } \boldsymbol{V}_{f}, \\
\rho_{m} g x_{3} \boldsymbol{e}_{3} \otimes \boldsymbol{e}_{3} & \text { in } \boldsymbol{V}_{m} .\end{cases}
\end{aligned}
$$

\section{Conclusion and perspectives}

We have shown that the effective behavior of a unidirectional composite material in the case where the fibers are debonded but still in contact with the matrix is formally similar to a generalized continuous medium whose kinematics contain not only the usual macroscopic displacement fields but also two scalar fields of internal variables describing the sliding and the rotation of the fibers. The two-scale procedure based on asymptotic expansions allowed us to formulate the effective problem giving the response of a composite body submitted to a mechanical loading. This problem can be formulated as the minimization of the effective potential energy of 
the composite body. This effective potential energy, difference of the effective elastic energy and the effective work of the applied forces, contains effective stiffness coefficients which are obtained by solving 12 elementary cell problems. Five of them can be solved in a closed form, the remaining seven requiring in general numerical computations. None of the problems are standard problems of the homogenization theory. Finally, the effective global problem leads to a system of coupled partial differential equations of second order which involve the kinematical fields.

The procedure was developed here in the particular case where the fibers and the matrix are linearly elastic isotropic materials with the assumption that the fibers remain in contact without friction with the matrix. We claim that it is possible to extend this work by removing some assumptions and enlarging the setting. For example, a first extension should be to consider prestresses in the composite and hence to develop the procedure in the case of an affine stress-strain relation. Another natural extension could be to consider more general and more realistic contact conditions between matrix and fibers: unilateral contact without friction or cohesive forces [Charlotte et al. 2006], for instance. The difficulty would be to solve nonlinear cell problems, and in such cases the effective behavior would no longer be described by a finite number of coefficients. An interesting mathematical challenge is to give a rigorous proof, by $\Gamma$-convergence for instance, that the effective behavior is really the one proposed here. It is a real issue because, as we have shown, the additional kinematical fields are less regular than the classical one. The consequences are that convergence could probably be proved only if the external forces satisfy certain smoothness conditions, and that the additional field should not satisfy arbitrary boundary conditions.

But the most interesting challenge is to introduce a law for the debonding evolution. Indeed, we have considered here that the domain where the fibers are debonded is given. But of course the real question is to find how this domain evolves with the loading. If we consider a Griffith-like assumption and suppose that debonding corresponds to an increase of the surface energy proportional to the new surface created [Bourdin et al. 2008], then the problem of debonding evolution will consist in finding when and how the potential energy is transformed into surface energy [Bilteryst and Marigo 2003]. If one adopts the global minimization principle proposed in [Francfort and Marigo 1993], then major mathematical difficulties will occur. Indeed, in the simplest case where the behavior of the material is described by two stiffness tensors, the damaged and the undamaged ones, it was shown in [Francfort and Marigo 1993] that the minimization energy problem does not admit classical solutions but must be relaxed to consider fine mixtures of damaged and undamaged material. In the present case the same phenomenon should probably also occur, but, because of the additional kinematical fields, its mathematical treatment should be much more difficult. 


\section{References}

[Abdelmoula and Marigo 2000] R. Abdelmoula and J.-J. Marigo, "The effective behavior of a fiber bridged crack", J. Mech. Phys. Solids 48:11 (2000), 2419-2444.

[Allaire 1992] G. Allaire, "Homogenization and two-scale convergence", SIAM J. Math. Anal. 23:6 (1992), 1482-1518.

[Bensoussan et al. 1978] A. Bensoussan, J.-L. Lions, and G. Papanicolaou, Asymptotic analysis for periodic structures, Studies in Mathematics and its Applications 5, North-Holland, Amsterdam, 1978.

[Berrehili and Marigo 2010] Y. Berrehili and J.-J. Marigo, "Modélisation en 2D du comportement d'un composite fibré à constituants décollés", Physical and Chemical News 53 (2010), 10-14.

[Bilteryst and Marigo 2003] F. Bilteryst and J.-J. Marigo, "An energy based analysis of the pull-out problem", Eur. J. Mech. A Solids 22:1 (2003), 55-69.

[Bouchelaghem et al. 2007] F. Bouchelaghem, A. Benhamida, and H. Dumontet, "Mechanical damage behaviour of an injected sand by periodic homogenization method", Computational Materials Science 38:3 (2007), 473-481.

[Bourdin et al. 2008] B. Bourdin, G. A. Francfort, and J.-J. Marigo, "The variational approach to fracture", J. Elasticity 91:1-3 (2008), 5-148.

[Caporale et al. 2006] A. Caporale, R. Luciano, and E. Sacco, "Micromechanical analysis of interfacial debonding in unidirectional fiber-reinforced composites", Computers \& Structures 84:31-32 (2006), 2200-2211.

[Charlotte et al. 2006] M. Charlotte, J. Laverne, and J.-J. Marigo, "Initiation of cracks with cohesive force models: A variational approach”, Eur. J. Mech. A Solids 25:4 (2006), 649-669.

[David et al. 2012] M. David, J.-J. Marigo, and C. Pideri, "Homogenized interface model describing inhomogeneities located on a surface", J. Elasticity 109:2 (2012), 153-187.

[Francfort and Marigo 1993] G. A. Francfort and J.-J. Marigo, "Stable damage evolution in a brittle continuous medium”, European J. Mech. A Solids 12:2 (1993), 149-189.

[Gonzàlez and LLorca 2007] C. Gonzàlez and J. LLorca, "Mechanical behavior of unidirectional fiber-reinforced polymers under transverse compression: Microscopic mechanisms and modeling", Composites Science and Technology 67:13 (2007), 2795-2806.

[Greco 2009] F. Greco, "Homogenized mechanical behavior of composite micro-structures including micro-cracking and contact evolution”, Engineering Fracture Mechanics 76:2 (2009), 182-208.

[Jendli et al. 2009] Z. Jendli, F. Meraghni, J. Fitoussi, and D. Baptiste, "Multi-scales modelling of dynamic behaviour for discontinuous fibre SMC composites", Composites Science and Technology 69:1 (2009), 97-103.

[Kulkarni et al. 2009] M. G. Kulkarni, P. H. Geubelle, and K. Matouš, "Multi-scale modeling of heterogeneous adhesives: Effect of particle decohesion”, Mechanics of Materials 41:5 (2009), 573583.

[Kushch et al. 2011] V. Kushch, S. Shmegera, and L. M. Jr., "Elastic interaction of partially debonded circular inclusions, II: Application to fibrous composite", Internat. J. Solids and Structures 48:1617 (2011), 2413-2421.

[Léné 1984] F. Léné, Contribution à l'étude des matériaux composites et de leur endommagement, thèse de doctorat d'état, Université Pierre et Marie Curie, Paris, 1984.

[Léné and Leguillon 1982] F. Léné and D. Leguillon, "Homogenized constitutive law for a partially cohesive composite material”, Internat. J. Solids and Structures 18:5 (1982), 443-458. 
[Marigo and Pideri 2011] J.-J. Marigo and C. Pideri, "The effective behavior of elastic bodies containing microcracks or microholes localized on a surface”, Int. J. Damage Mech. 20:8 (2011), 11511171.

[Marigo et al. 1987] J.-J. Marigo, P. Mialon, J.-C. Michel, and P. Suquet, "Plasticité et homogénéisation: Un exemple de prévision des charges limites d'une structure hétérogène périodique", J. Méc. Théor. Appl. 6 (1987), 47-75.

[Matouš and Geubelle 2006] K. Matouš and P. H. Geubelle, "Multiscale modelling of particle debonding in reinforced elastomers subjected to finite deformations", Internat. J. Numer. Methods Engrg. 65:2 (2006), 190-223.

[Michel et al. 1999] J. C. Michel, H. Moulinec, and P. Suquet, "Effective properties of composite materials with periodic microstructure: A computational approach", Comput. Methods Appl. Mech. Engrg. 172:1-4 (1999), 109-143.

[Moraleda et al. 2009] J. Moraleda, J. Segurado, and J. Llorca, "Effect of interface fracture on the tensile deformation of fiber-reinforced elastomers", Internat. J. Solids and Structures 46:25-26 (2009), 4287-4297.

[Sánchez-Palencia 1980] E. Sánchez-Palencia, Nonhomogeneous media and vibration theory, Lecture Notes in Physics 127, Springer, Berlin, 1980.

[Suquet 1982] P. Suquet, Plasticité et homogénéisation, thèse de doctorat d'état, Université Pierre et Marie Curie, Paris, 1982.

[Teng 2010] H. Teng, "Stiffness properties of particulate composites containing debonded particles", Internat. J. Solids and Structures 47:17 (2010), 2191-2200.

Received 31 Dec 2012. Revised 7 Apr 2013. Accepted 19 Jun 2013.

YAHYA BERREHILI: yberrehili@ensa.ump.ma

Equipe de Modélisation et Simulation Numérique, Université Mohamed 1er, Ecole Nationale des Sciences Appliquées, 60000 Oujda, Morocco

JEAN-JACQUES MARIGO: marigo@lms .polytechnique.fr

Laboratoire de Mécanique des Solides, École Polytechnique, CNRS, UMR 7649,

91128 Palaiseau cedex, France 



\title{
STATISTICALLY ISOTROPIC TENSOR RANDOM FIELDS: CORRELATION STRUCTURES
}

\author{
Anatoliy Malyarenko AND Martin OStojA-STARZEWSKi
}

Let $V$ be a real finite-dimensional vector space. We introduce some physical problems that may be described by $V$-valued homogeneous and isotropic random fields on $\mathbb{R}^{3}$. We propose a general method for calculation of expectations and two-point correlation functions of such fields. Our results are equivalent to classical results by Robertson, when $V=\mathbb{R}^{3}$, and those by Lomakin, when $V$ is the space of symmetric second-rank tensors over $\mathbb{R}^{3}$. Our solution involves an analogue of the classical Clebsch-Gordan coefficients.

\section{Introduction}

The entire field of continuum physics involves tensor fields. Overwhelmingly, most of the existing models and theories are deterministic and their stochastic generalizations necessitate construction of tensor-valued random fields (RF). While the literature on scalar RFs is vast (for example, [Cressie 1993; Christakos 2005; Marinucci and Peccati 2011; Leonenko and Sakhno 2012; Porcu et al. 2012]), that on vector RFs is largely limited to statistical turbulence [Monin and Yaglom 1965], and the case of higher tensor rank (second, fourth) RFs poses challenges. In this paper we focus on wide-sense stationary and statistically isotropic RFs of tensors of the first and second ranks. We present a new method of derivation of representations of their correlation functions, which in the case of first-rank tensors gives the same result as in [Robertson 1940], while in the case of second-rank tensors is equivalent to the result of [Lomakin 1964].

These representations have applications to tensor random fields (TRFs) governed by the field equations of continuum physics as well as those representing some spatially inhomogeneous constitutive properties of random media. The former type of TRFs is used in [Ostoja-Starzewski et al. 2013], where correlation functions are subject to constraints such as the equilibrium equation or straindisplacement relation. The basic properties of TRFs of a wide-sense homogeneous and isotropic kind with generally anisotropic realizations have been determined

\section{Communicated by Eric A. Carlen.}

MSC2010: primary 60G60; secondary 74A40.

Keywords: isotropic tensor random field, group representation, Godunov-Gordienko coefficients. 
in three continuum physics theories: thermal conduction, classical elasticity, and micropolar elasticity. The field equations (such as the linear and angular momentum balances and strain-displacement relations), all in a quasistatic setting, lead to consequences for the respective dependent fields involved. In effect, these consequences are restrictions on the admissible forms of the correlation functions describing the TRFs.

The latter type of TRFs provides models of random media described by the second-rank TRF. The typical example here is the thermal conductivity tensor and its mathematical analogies such as the antiplane stiffness tensor. Once the general representation of this TRF is established and the conditions of positive definiteness are imposed, one can turn to modeling and simulation of the entire range of statistical constitutive behaviors of all heat-conducting media or, say, elastic materials subjected to antiplane loading, for example, [Sena et al. 2013].

In particular, let $V$ be a finite-dimensional real Hilbert space with norm $\|\cdot\|$. Let $\boldsymbol{T}(\boldsymbol{x}), \boldsymbol{x} \in \mathbb{R}^{3}$, be a random field taking values in (a subset of) $V$. Suppose that $\mathrm{E}\left[\|\boldsymbol{T}(\boldsymbol{x})\|^{2}\right]<\infty$ and that $\boldsymbol{T}(\boldsymbol{x})$ is mean-square continuous, that is, for any $\boldsymbol{x}_{0} \in \mathbb{R}^{3}$ we have

$$
\lim _{\left\|\boldsymbol{x}-\boldsymbol{x}_{0}\right\| \rightarrow 0} \mathrm{E}\left[\left\|\boldsymbol{T}(\boldsymbol{x})-\boldsymbol{T}\left(\boldsymbol{x}_{0}\right)\right\|^{2}\right]=0 .
$$

Let $E(\boldsymbol{x})=\mathrm{E}[\boldsymbol{T}(\boldsymbol{x})]$ be the expectation of the field, and let $B(\boldsymbol{x}, \boldsymbol{y})=\mathrm{E}[\boldsymbol{T}(\mathbf{x}) \otimes$ $\boldsymbol{T}(\boldsymbol{y})]$ be the two-point correlation function of the random field $\boldsymbol{T}(\boldsymbol{x})$. The group $\mathbb{R}^{3}$ acts on itself by translations. Assume that the above functions are invariant with respect to this action, that is, for all $\boldsymbol{x}, \boldsymbol{y}, \boldsymbol{z} \in \mathbb{R}^{3}$,

$$
\begin{aligned}
E(x+z) & =E(x), \\
B(x+z, y+z) & =B(x, y) .
\end{aligned}
$$

It follows that $E(\boldsymbol{x})=\boldsymbol{E} \in V$ is constant, while $B(\boldsymbol{x}, \boldsymbol{y}) \in V \otimes V$ depends only on the difference $\boldsymbol{x}-\boldsymbol{y}$.

Let $K=\mathrm{SO}(3)$ be the group of rotations in $\mathbb{R}^{3}$, and let $(V, \gamma)$ be an orthogonal representation of $K$. Suppose that for all $k \in K$ and all $x \in \mathbb{R}^{3}$ we have

$$
\begin{aligned}
& E(k \boldsymbol{x})=\gamma(k) E(\boldsymbol{x}), \\
& B(k \boldsymbol{x})=\gamma(k) B(\boldsymbol{x}) \gamma^{-1}(k) .
\end{aligned}
$$

We would like to find a general form for the expectation and two-point correlation function of such a field.

In Section 2, we consider mathematical preliminaries. We use the book [Adams 1969], in which Adams considers both real and complex representations at the same time.

In Section 3 we consider two particular cases of the above problem: 
(1) $V$ has dimension 3 and $\gamma(k)=k$.

(2) $V$ is the space of all second-rank tensors over $\mathbb{R}^{3}, \boldsymbol{T}(\boldsymbol{x})$ takes values in the set of all symmetric tensors, and the representation is $\gamma(k) \boldsymbol{T}=k \boldsymbol{T} k^{-1}$.

In the first case, the answer has been known since the classic paper [Robertson 1940]. In Theorem 3.1, we prove that our method of solution gives the same answer. Our new result is Theorem 3.2. Section 4 concludes. Proofs of our results are collected in the Appendix.

\section{Mathematical preliminaries}

Let $\mathbb{K}$ be either the field $\mathbb{R}$ of real numbers or the field $\mathbb{C}$ of complex numbers, and let $K$ be a topological group with the identity element $e$. A representation of the group $K$ over $\mathbb{K}$ is a pair $(V, \gamma)$, where $V$ is a finite-dimensional vector space over $\mathbb{K}$, and $\gamma$ is a continuous homomorphism from $K$ to the group Aut $V$ of the invertible linear operators in $V$. In other words, for each $k \in K$ and for each $v \in V$ there is a vector $\gamma(k) v \in V$, and the following conditions hold true:

(1) $\gamma(e) \boldsymbol{v}=\boldsymbol{v}$ and $\gamma(k)\left(\gamma\left(k^{\prime}\right) \boldsymbol{v}\right)=\gamma\left(k k^{\prime}\right) \boldsymbol{v}$.

(2) $\gamma(k) \boldsymbol{v}$ is a $\mathbb{K}$-linear function of $\boldsymbol{v}$.

(3) $\gamma(k) v$ is a continuous function of $k$ and $\boldsymbol{v}$.

Let $(V, \gamma)$ and $(W, \delta)$ be two representations. A map $G: V \rightarrow W$ is called a $K$-map if

$$
G(\gamma(k) v)=\delta(k)(G \boldsymbol{v}) .
$$

A $\mathbb{K}$-linear $K$-map is called an intertwining operator. The set of all intertwining operators is a vector space over $\mathbb{K}$. The representations $(V, \gamma)$ and $(W, \delta)$ are called equivalent if the above space contains an invertible operator.

Let $(V, \gamma)$ be a real representation of the group $K$. Build a complex representation $\left(V^{\prime}, \gamma^{\prime}\right)$ as follows. Consider $\mathbb{C}$ as a vector space over $\mathbb{R}$. Put $V^{\prime}=\mathbb{C} \otimes_{\mathbb{R}} V$. The space $V^{\prime}$ is a complex vector space, where multiplication by a complex number $z$ is defined as $z\left(z^{\prime} \otimes \boldsymbol{v}\right)=z z^{\prime} \otimes \boldsymbol{v}$. The representation $\gamma^{\prime}$ is

$$
\gamma^{\prime}(k)(z \otimes \boldsymbol{v})=z \otimes \gamma(k) \boldsymbol{v} .
$$

Define a map $j: V^{\prime} \rightarrow V^{\prime}$ by $j(z \otimes v)=\bar{z} \otimes \boldsymbol{v}$. Then $j$ is a structural map, that is, a $K$-map with

$$
j(z \boldsymbol{v})=\bar{z} j(\boldsymbol{v}), \quad j^{2}=1 .
$$

Conversely, let $\left(V^{\prime}, \gamma^{\prime}\right)$ be a complex representation of $K$ that admits a structural map $j$. Then $V^{\prime}$ is a direct sum of two eigenspaces $V_{+}$and $V_{-}$of the map $j$ that correspond to the eigenvalues +1 and -1 . These spaces carry two equivalent real 
representations. Multiplication by $\mathrm{i}$ is an invertible intertwining operator between the above representations.

The direct sum of two representations $(V, \gamma)$ and $(W, \delta)$ is the representation $(V \oplus W, \gamma \oplus \delta)$, where

$$
\gamma \oplus \delta(k)(\boldsymbol{v} \oplus \boldsymbol{w})=(\gamma(k) \boldsymbol{v}) \oplus(\delta(k) \boldsymbol{w}) .
$$

The tensor product of two representations $(V, \gamma)$ and $(W, \delta)$ is the representation $(V \otimes W, \gamma \otimes \delta)$, where

$$
\gamma \otimes \delta(k)(\boldsymbol{v} \otimes \boldsymbol{w})=(\gamma(k) \boldsymbol{v}) \otimes(\delta(k) \boldsymbol{w}) .
$$

A representation $(V, \gamma)$ with $V \neq\{\mathbf{0}\}$ is called reducible if there exists a proper subspace $W$ of $V$ with $\gamma(k) \boldsymbol{w} \in W$ for all $\boldsymbol{w} \in W$ and $k \in K$ and irreducible otherwise. If $K$ is a compact group, then any representation $(V, \gamma)$ of $K$ is a direct sum of irreducible representations. Moreover, the decomposition onto irreducible representations is unique in the following sense. If $m_{i} V_{i}$ denotes the direct sum of $m_{i}$ copies of the representation $V_{i}$, and the representations $\bigoplus m_{i} V_{i}$ and $\bigoplus n_{i} V_{i}$ are equivalent, then $m_{i}=n_{i}$ for all $i$.

Let $(V, \gamma)$ be a complex representation of a compact topological group $K$. By [Adams 1969, Proposition 3.16], there exists a $K$-invariant inner product $(\cdot, \cdot)$ on $V$. Moreover, if $(V, \gamma)$ admits a structural map $j$, one can choose the above inner product in such a way that $(j \boldsymbol{v}, j \boldsymbol{w})=\overline{(\boldsymbol{v}, \boldsymbol{w})}$, and the restriction of the inner product to either space $V_{+}$or $V_{-}$is again an inner product.

Choose an orthonormal basis $\boldsymbol{e}_{1}, \ldots, \boldsymbol{e}_{n}$ in $V$. Then, the complex representation $\gamma$ takes values in the unitary group $U(n)$ and is called a unitary representation. A real representation takes values in the orthogonal group $O(n)$ and is called an orthogonal representation.

Realize $\mathbb{R}^{3}$ as the space of traceless Hermitian matrices in $\mathbb{C}^{2}$. Such a matrix has the form

$$
A=\left(\begin{array}{cc}
x_{0} & x_{1}+x_{-1} \mathrm{i} \\
x_{1}-x_{-1} \mathrm{i} & -x_{0}
\end{array}\right), \quad x_{-1}, x_{0}, x_{1} \in \mathbb{R} .
$$

The map $A \mapsto k^{-1} A k$, where $k$ is an element of the group SU(2) of unitary $2 \times 2$ matrices with unit determinant, is a rotation, that is, an element of the group $\mathrm{SO}(3)$. The matrices $k$ and $-k$ determine the same rotation. Conversely, each rotation in $\mathrm{SO}(3)$ corresponds to a pair of matrices $k$ and $-k$.

Let $(V, \gamma)$ be an irreducible unitary representation of the group $\mathrm{SU}(2)$. If $\gamma(k)=$ $\gamma(-k)$, then $(V, \gamma)$ is an irreducible unitary representation of the group $\operatorname{SO}(3)$, and all irreducible unitary representations of $\mathrm{SO}(3)$ may be obtained in this way. 
Let

$$
k=\left(\begin{array}{rr}
\alpha & \beta \\
-\bar{\beta} & \bar{\alpha}
\end{array}\right), \quad \alpha, \beta \in \mathbb{C}, \quad|\alpha|^{2}+|\beta|^{2}=1,
$$

be an element of the group SU(2). Let $V^{\ell}$ be the space of homogeneous polynomials of degree $2 \ell$ in two complex variables $\xi$ and $\eta$. The representation

$$
\gamma_{\ell}(k) f(\xi, \eta)=f(\bar{\alpha} \xi-\beta \eta, \bar{\beta} \xi+\alpha \eta)
$$

is irreducible. Conversely, any irreducible representation of the group $\mathrm{SU}(2)$ is equivalent to the representation (2-1).

If $\ell$ is an integer, then $\gamma_{\ell}(k)=\gamma_{\ell}(-k)$, and the representation (2-1) is an irreducible representation of the group $\mathrm{SO}(3)$. Moreover, put

$$
j f(\xi, \eta)=\bar{f}(-\eta, \xi)
$$

where $\bar{f}$ is the polynomial with coefficients which are complex conjugate to those of $f$. Then $j$ is a structural map. If we choose an orthonormal basis $\boldsymbol{e}_{m}^{\ell},-\ell \leq m \leq \ell$, satisfying the condition

$$
j \boldsymbol{e}_{m}^{\ell}=\boldsymbol{e}_{m}^{\ell},
$$

then the restriction $\left(V_{+}^{\ell}, \gamma_{\ell,+}\right)$ of the representation $\left(V^{\ell}, \gamma_{\ell}\right)$ to the real linear span $V_{+}^{\ell}$ of the above basis is an irreducible real representation, and the matrix entries of the operators $\gamma_{\ell}(k)$ are real-valued functions on the group $\mathrm{SO}(3)$. If the basis $\boldsymbol{e}_{m}^{\ell}$ satisfies the condition

$$
j \boldsymbol{e}_{m}^{\ell}=-\boldsymbol{e}_{m}^{\ell}
$$

then the restriction $\left(V_{-}^{\ell}, \gamma_{\ell,-}\right)$ of the representation $\left(V^{\ell}, \gamma_{\ell}\right)$ to the real linear span $V_{-}^{\ell}$ of the above basis is an irreducible real representation, equivalent to $\left(V_{+}^{\ell}, \gamma_{\ell,+}\right)$, and multiplication by $\mathrm{i}$ is an orthogonal intertwining operator between two equivalent representations.

The usual orthonormal basis in the space $V^{\ell}$ is as follows:

$$
\boldsymbol{f}_{m}^{\ell}(\xi, \eta)=(-1)^{\ell+m} \sqrt{\frac{(2 \ell+1) !}{(\ell+m) !(\ell-m) !}} \xi^{\ell+m} \eta^{\ell-m}
$$

The matrix entries of operators $\gamma_{\ell}(k)$ in this basis are called the Wigner D-functions and are denoted by $D_{m n}^{\ell}(k)$. The basis (2-4) does not satisfy (2-2). Gordienko 
[2002] proposed the basis satisfying (2-2) as follows:

$$
\begin{aligned}
\boldsymbol{h}_{-m}^{\ell}(\xi, \eta) & =\frac{(-\mathrm{i})^{\ell-1}}{\sqrt{2}}\left[(-1)^{m} \boldsymbol{f}_{m}^{\ell}(\xi, \eta)-\boldsymbol{f}_{-m}^{\ell}(\xi, \eta)\right], \\
\boldsymbol{h}_{0}^{\ell}(\xi, \eta) & =(-\mathrm{i})^{\ell} \boldsymbol{e}_{0}^{\ell}(\xi, \eta), \\
\boldsymbol{h}_{m}^{\ell}(\xi, \eta) & =-\frac{(-\mathrm{i})^{\ell}}{\sqrt{2}}\left[(-1)^{m} \boldsymbol{f}_{m}^{\ell}(\xi, \eta)+\boldsymbol{f}_{-m}^{\ell}(\xi, \eta)\right],
\end{aligned}
$$

where $m \geq 1$. From now on, we define by $U^{\ell}(k)$ the matrices of the representation $\left(V_{+}^{\ell}, \gamma_{\ell,+}\right)$ in the Gordienko basis and omit + and - for simplicity of notation. Note that $\mathrm{i} \boldsymbol{h}_{m}^{\ell}(\xi, \eta)$ is the Gordienko basis of the space $V_{-}$. Its vectors satisfy (2-3).

Any rotation $k$ may be performed by three successive rotations:

- rotation $k_{0}(\psi)$ about the $x_{0}$-axis through an angle $\psi, 0 \leq \psi<2 \pi$,

- rotation $k_{-1}(\theta)$ about the $x_{-1}$-axis through an angle $\theta, 0 \leq \theta \leq \pi$, and

- rotation $k_{0}(\varphi)$ about the $x_{0}$-axis through an angle $\varphi, 0 \leq \varphi<2 \pi$.

The angles $\psi, \theta$, and $\varphi$ are the Euler angles. The map which maps the product of the above rotations $k(\psi, \theta, \varphi)$ to the point $(\psi, \theta, \varphi) \in(0,2 \pi) \times(0, \pi) \times(0,2 \pi)$ is a chart of the group manifold $\mathrm{SO}(3)$, and the domain of this chart is an open dense subset of $\mathrm{SO}(3)$. Moreover, the map which maps the rotation $k(0, \theta, \varphi)(0,1,0)^{\top}$ to the point $(\theta, \varphi) \in(0, \pi) \times(0,2 \pi)$ is a chart of the unit sphere $S^{2}$ centered at the origin of the space $\mathbb{R}^{3}$. The coordinates of the point $k(0, \theta, \varphi)(0, r, 0)^{\top}, r>0$, are the spherical coordinates,

$$
\begin{aligned}
x_{-1} & =r \sin \varphi \sin \theta, \\
x_{0} & =r \cos \theta, \\
x_{1} & =r \cos \varphi \sin \theta,
\end{aligned}
$$

Gordienko [2002] calculated the matrix entries of the matrices $U^{\ell}(k)$. His result is as follows. If $k=k(\psi, \theta, \varphi)$, then

$$
U^{\ell}(k)=U^{\ell}\left(k_{0}(\varphi)\right) U^{\ell}\left(k_{-1}(\theta)\right) U^{\ell}\left(k_{0}(\psi)\right),
$$

by the definition of a representation. Denote the matrix entries of the matrix $U^{\ell}\left(k_{0}(\varphi)\right)$ by $\Omega_{0, m, n}^{\ell}(\varphi)$, where $-\ell \leq m, n \leq \ell$. The nonzero entries are

$$
\Omega_{0,0,0}^{\ell}(\varphi)=1, \quad \Omega_{0, m, m}^{\ell}(\varphi)=\cos (m \varphi), \quad \Omega_{0,-m, m}^{\ell}(\varphi)=\sin (m \varphi),
$$


where $m= \pm 1, \pm 2, \ldots, \pm \ell$. Denote the matrix entries of the matrix $U^{\ell}\left(k_{-1}(\theta)\right)$ by $\Omega_{-1, m, n}^{\ell}(\theta)$. The nonzero entries are

$$
\begin{aligned}
\Omega_{-1, \pm m, \pm n}^{\ell}(\theta)= & \frac{(-1)^{\ell-n}}{2^{\ell}\left(1-\mu^{2}\right)^{n / 2}} \sqrt{\frac{(\ell+n) !}{(\ell-n) !(\ell-m) !(\ell+m) !}} \\
& \times\left\{\left(\frac{1-\mu}{1+\mu}\right)^{n / 2} \frac{\mathrm{d}^{\ell-n}}{\mathrm{~d} \mu^{\ell-n}}\left[(1+\mu)^{\ell+m}(1-\mu)^{\ell-m}\right]\right. \\
& \left. \pm(-1)^{m}\left(\frac{1+\mu}{1-\mu}\right)^{n / 2} \frac{\mathrm{d}^{\ell-n}}{\mathrm{~d} \mu^{\ell-n}}\left[(1+\mu)^{\ell-m}(1-\mu)^{\ell+m}\right]\right\} \\
\Omega_{-1,0,0}^{\ell}(\theta)= & \frac{(-1)^{\ell}}{2^{\ell} \ell !} \frac{\mathrm{d}^{\ell}}{\mathrm{d} \mu^{\ell}}\left(1-\mu^{2}\right)^{\ell} \\
\Omega_{-1,0, n}^{\ell}(\theta)= & \frac{(-1)^{\ell-n}}{2^{\ell} \ell !} \sqrt{\frac{2(\ell+n) !}{(\ell-n) !}} \frac{1}{\left(1-\mu^{2}\right)^{n / 2}} \frac{\mathrm{d}^{\ell-n}}{\mathrm{~d} \mu^{\ell-n}}\left(1-\mu^{2}\right)^{\ell} \\
\Omega_{-1, m, 0}^{\ell}(\theta)=- & \frac{(-1)^{\ell}}{2^{\ell} \ell !} \sqrt{\frac{2(\ell+m) !}{(\ell-m) !}} \frac{1}{\left(1-\mu^{2}\right)^{m / 2}} \frac{\mathrm{d}^{\ell-m}}{\mathrm{~d} \mu^{\ell-m}}\left(1-\mu^{2}\right)^{\ell}
\end{aligned}
$$

where $m \geq 1, n \geq 1$, and where $\mu=\cos \theta$.

Let $\left(V^{m}, \gamma_{m}\right)$ and $\left(V^{p}, \gamma_{p}\right)$ be two irreducible orthogonal representations of the group $\mathrm{SO}(3)$. Their tensor product $\left(V^{m} \otimes V^{p}, \gamma_{m} \otimes \gamma_{p}\right)$ is equivalent to the direct $\operatorname{sum}\left(V^{|m-p|} \oplus V^{|m-p|+1} \oplus \cdots \oplus V^{m+p}, \gamma_{|m-p|} \oplus \gamma_{|m-p|+1} \oplus \cdots \oplus \gamma_{m+p}\right)$. Let $G$ be the orthogonal intertwining operator between the above equivalent representations. Then we have

$$
G\left(\gamma_{m}(k) \otimes \gamma_{p}(k)\right)=\left(\gamma_{|m-p|(k)} \oplus \gamma_{|m-p|+1}(k) \oplus \cdots \oplus \gamma_{m+p}(k)\right) G .
$$

In the usual basis (2-4), this equality takes the form

$$
\sum_{i=-m}^{m} \sum_{j=-p}^{p} C_{m i p j}^{s \ell} D_{n i}^{m}(k) D_{q j}^{p}(k)=\sum_{t=-s}^{s} U_{t \ell}^{s}(k) C_{m n p q}^{s t},
$$

which is [Varshalovich et al. 1975, Equation (5), §4.6]. The matrix entries of the operator $G$ in the basis (2-4), $C_{m i p j}^{s \ell}$, are called the Clebsch-Gordan coefficients.

In the Gordienko basis, the same equality takes the form

$$
\sum_{i=-m}^{m} \sum_{j=-p}^{p} g_{s[m, p]}^{\ell[i, j]} U_{n i}^{m}(k) U_{q j}^{p}(k)=\sum_{t=-s}^{s} U_{t \ell}^{s}(k) g_{s[m, p]}^{t[n, q]} .
$$

We call the matrix entries of the operator $G$ in the Gordienko basis, $g_{s[m, p]}^{\ell[i, j]}$, the 
Godunov-Gordienko coefficients. They were calculated in [Godunov and Gordienko 2004].

It follows in particular that the matrix $G$ transforms the uncoupled basis $\boldsymbol{h}_{t}^{s}$, $|m-p| \leq s \leq m+p,-s \leq t \leq s$, to the coupled basis $\boldsymbol{h}_{n}^{m} \otimes \boldsymbol{h}_{q}^{p}$ :

$$
\boldsymbol{h}_{n}^{m} \otimes \boldsymbol{h}_{q}^{p}=\sum_{r=|m-p|}^{m+p} \sum_{s=-r}^{r} g_{r[m, p]}^{s[n, q]} \boldsymbol{h}_{s}^{r}
$$

We multiply both sides of (2-8) by $G^{-1}$ from the left and write the result in the Gordienko basis. We obtain

$$
U_{n i}^{m}(k) U_{q j}^{p}(k)=\sum_{s=|m-p|}^{m+p} \sum_{t=-s}^{s} \sum_{\ell=-s}^{s} g_{s[m, p]}^{t[n, q]} U_{t \ell}^{s}(k) g_{s[m, p]}^{\ell[i, j]} .
$$

The same equality in the usual basis is [Varshalovich et al. 1975, Equation (1), $\S 4.6]$. It is called the Clebsch-Gordan expansion.

Lemma 2.1 [Malyarenko 2013]. Let $U$ be an irreducible representation of a topological group $K$ in a Hilbert space $H$. Let $\boldsymbol{x} \in H$ be a common eigenvector of all operators $U(k), g \in K$. If $U$ is not trivial, then $\boldsymbol{x}=\mathbf{0}$.

Lemma 2.2. The second equation in (1-1) may be written in the Gordienko basis as follows:

$$
B(k \boldsymbol{x})=(U \otimes U)(k) B(\boldsymbol{x})
$$

\section{The results}

Theorem 3.1 [Robertson 1940]. Let $\boldsymbol{T}(\boldsymbol{x})$ be a $V$-valued random field on $\mathbb{R}^{3}$ satisfying (1-1) with $U(k)=k$. Then

$$
\mathrm{E}[\boldsymbol{T}(\boldsymbol{x})]=\mathbf{0}
$$

and there exist two continuous functions $K_{0}, K_{2}:[0, \infty) \rightarrow \mathbb{R}$ with $K_{2}(0)=0$ such that

$$
B_{i j}(\boldsymbol{x})=\delta_{i j} K_{0}(\|\boldsymbol{x}\|)+x_{i} x_{j} K_{2}(\|\boldsymbol{x}\|) .
$$

Let $k_{\boldsymbol{x}}, \boldsymbol{x} \neq \mathbf{0}$, be the rotation with Euler angles $(0, \theta, \varphi)$, where $\theta$ and $\varphi$ are angular spherical coordinates of the point $\boldsymbol{x}$. We introduce the following notation: 


$$
\begin{aligned}
& M_{i j \ell m}^{1}(\boldsymbol{x})=\frac{1}{3} \delta_{i j} \delta_{\ell m}, \\
& M_{i j \ell m}^{2}(\boldsymbol{x})=\frac{1}{\sqrt{5}} \sum_{n=-2}^{2} g_{2[1,1]}^{n[i, j]} g_{2[1,1]}^{n[\ell, m]}, \\
& M_{i j \ell m}^{3}(\boldsymbol{x})=\frac{1}{\sqrt{6}}\left[\delta_{i j} \sum_{n=-2}^{2} g_{2[1,1]}^{n[\ell, m]} U_{n 0}^{2}\left(k_{\boldsymbol{x}}\right)+\delta_{\ell m} \sum_{n=-2}^{2} g_{2[1,1]}^{n[i, j]} U_{n 0}^{2}\left(k_{\boldsymbol{x}}\right)\right], \\
& M_{i j \ell m}^{4}(\boldsymbol{x})=\sum_{n=-2}^{2} \sum_{p=-2}^{2} g_{2[1,1]}^{n[i, j]} g_{2[1,1]}^{p[\ell, m]} \sum_{q=-2}^{2} g_{2[2,2]}^{q[n, p]} U_{q 0}^{2}\left(k_{\boldsymbol{x}}\right), \\
& M_{i j \ell m}^{5}(\boldsymbol{x})=\sum_{n=-2}^{2} \sum_{p=-2}^{2} g_{2[1,1]}^{n[i, j]} g_{2[1,1]}^{p[\ell, m]} \sum_{q=-4}^{4} g_{4[2,2]}^{q[n, p]} U_{q 0}^{4}\left(k_{\boldsymbol{x}}\right) .
\end{aligned}
$$

Theorem 3.2. Let $V$ be the space of all symmetric second-rank tensors over $\mathbb{R}^{3}$, let $\boldsymbol{T}(\boldsymbol{x})$ be a $V$-valued random field on $\mathbb{R}^{3}$ satisfying (1-1) with $U(k) \boldsymbol{T}=k \boldsymbol{T} k^{-1}$. Then $E_{i j}(\boldsymbol{x})=C \delta_{i j}, C \in \mathbb{R}$, and there exist five continuous functions $K_{1}, \ldots, K_{5}$ : $[0, \infty) \rightarrow \mathbb{R}$ with $K_{3}(0)=K_{4}(0)=K_{5}(0)=0$ such that

$$
B_{i j \ell m}(\boldsymbol{x})=\sum_{n=1}^{5} M_{i j \ell m}^{n}(\boldsymbol{x}) K_{n}(\|\boldsymbol{x}\|)
$$

A formula similar to (3-2) has been obtained by Lomakin [1964]. For any fixed $\boldsymbol{x} \in \mathbb{R}^{3}$, the tensor in the left-hand side of (3-2) is a symmetric linear operator acting in the space of symmetric tensors of the second rank. Following Boehler et al. [1994], denote the space of all such tensors by $T_{4}^{e}$. Under the action of $\mathrm{SO}(3)$, the space $T_{4}^{e}$ decomposes into the following direct sum:

$$
T_{4}^{e}=V^{0} \oplus V^{0} \oplus V^{2} \oplus V^{2} \oplus V^{4},
$$

where $V^{i}$ may be considered as the space of completely symmetric traceless tensors of the $i$-th rank linearly dependent on $x_{i}$. Using the general form of such a tensor given by invariant theory (see, for example, [Spencer 1971]), we obtain the result of [Lomakin 1964]:

$$
B_{i j \ell m}(\boldsymbol{x})=\sum_{n=1}^{5} L_{i j \ell m}^{n}(\boldsymbol{x}) K_{n}(\|\boldsymbol{x}\|),
$$

where (compare with [Boehler et al. 1994, Lemma, pp. 98-99]) 


$$
\begin{aligned}
L_{i j \ell m}^{1}(\boldsymbol{x}) & =\delta_{i j} \delta_{\ell m}, \\
L_{i j \ell m}^{2}(\boldsymbol{x}) & =\delta_{i \ell} \delta_{j m}+\delta_{i m} \delta_{j l}, \\
L_{i j \ell m}^{3}(\boldsymbol{x}) & =\frac{x_{j} x_{\ell}}{\|\boldsymbol{x}\|^{2}} \delta_{i m}+\frac{x_{i} x_{m}}{\|\boldsymbol{x}\|^{2}} \delta_{j \ell}+\frac{x_{i} x_{\ell}}{\|\boldsymbol{x}\|^{2}} \delta_{j m}+\frac{x_{j} x_{m}}{\|\boldsymbol{x}\|^{2}} \delta_{i \ell}, \\
L_{i j \ell m}^{4}(\boldsymbol{x}) & =\frac{x_{i} x_{j}}{\|\boldsymbol{x}\|^{2}} \delta_{\ell m}+\frac{x_{\ell} x_{m}}{\|\boldsymbol{x}\|^{2}} \delta_{i j}, \\
L_{i j \ell m}^{5}(\boldsymbol{x}) & =\frac{x_{i} x_{j} x_{\ell} x_{m}}{\|\boldsymbol{x}\|^{4}} .
\end{aligned}
$$

We prove that Theorem 3.2 is equivalent to the result of [Lomakin 1964]. Indeed, we have

$$
\begin{aligned}
M_{i j \ell m}^{1}(\boldsymbol{x}) & =\frac{1}{3} L_{i j \ell m}^{1}(\boldsymbol{x}), \\
M_{i j \ell m}^{2}(\boldsymbol{x}) & =-\frac{1}{3 \sqrt{5}} L_{i j \ell m}^{1}(\boldsymbol{x})+\frac{1}{2 \sqrt{5}} L_{i j \ell m}^{2}(\boldsymbol{x}), \\
M_{i j \ell m}^{3}(\boldsymbol{x}) & =-\frac{1}{3} L_{i j \ell m}^{1}(\boldsymbol{x})+\frac{1}{2} L_{i j \ell m}^{4}(\boldsymbol{x}), \\
M_{i j \ell m}^{4}(\boldsymbol{x}) & =\frac{2 \sqrt{2}}{3 \sqrt{7}} L_{i j \ell m}^{1}(\boldsymbol{x})-\frac{1}{\sqrt{14}} L_{i j \ell m}^{2}(\boldsymbol{x})+\frac{3}{2 \sqrt{14}} L_{i j \ell m}^{3}(\boldsymbol{x})-\frac{\sqrt{2}}{\sqrt{7}} L_{i j \ell m}^{4}(\boldsymbol{x}), \\
M_{i j \ell m}^{5}(\boldsymbol{x}) & =\frac{1}{2 \sqrt{70}} L_{i j \ell m}^{1}(\boldsymbol{x})+\frac{1}{2 \sqrt{70}} L_{i j \ell m}^{2}(\boldsymbol{x})-\frac{\sqrt{5}}{2 \sqrt{14}} L_{i j \ell m}^{3}(\boldsymbol{x}) \\
& -\frac{\sqrt{5}}{2 \sqrt{14}} L_{i j \ell m}^{4}(\boldsymbol{x})+\frac{\sqrt{35}}{2 \sqrt{2}} L_{i j \ell m}^{5}(\boldsymbol{x}) .
\end{aligned}
$$

It is easy to check that the transition matrix between Lomakin's functions (3-3) and the functions (3-1) is invertible. A proof of (3-4) may be found in the Appendix.

Given that $\boldsymbol{T}$ has diagonal and off-diagonal components, there are five special cases of $B_{i j \ell m}$ that shed light on the physical meaning of the $K_{n}$ :

(1) $\left.\mathrm{E}\left[T_{i j}(\mathbf{0}) T_{k l}(\boldsymbol{x})\right]\right|_{i=j=k=l}$; that is, auto-correlations of diagonal terms

$$
\mathrm{E}\left[T_{11}(\mathbf{0}) T_{11}(\boldsymbol{x})\right]=K_{1}+2 K_{2}+2 x_{1}^{2} K_{3}+4 x_{1}^{2} K_{4}+x_{1}^{4} K_{5}
$$

and then $\mathrm{E}\left[T_{22}(\mathbf{0}) T_{22}(\boldsymbol{x})\right]$ and $\mathrm{E}\left[T_{33}(\mathbf{0}) T_{33}(\boldsymbol{x})\right]$ by cyclic permutations $1 \rightarrow$ $2 \rightarrow 3$.

(2) $\left.\mathrm{E}\left[T_{i j}(\mathbf{0}) T_{k l}(\boldsymbol{x})\right]\right|_{i=j \neq k=l}$; that is, cross-correlations of diagonal terms

$$
\mathrm{E}\left[T_{11}(\mathbf{0}) T_{22}(\boldsymbol{x})\right]=K_{1}+\left(x_{2}^{2}+x_{1}^{2}\right) K_{3}+x_{2}^{2} x_{1}^{2} K_{5}
$$

and then $\mathrm{E}\left[T_{22}(\mathbf{0}) T_{33}(\boldsymbol{x})\right]$ and $\mathrm{E}\left[T_{33}(\mathbf{0}) T_{11}(\boldsymbol{x})\right]$ by cyclic permutations $1 \rightarrow$ $2 \rightarrow 3$. 
(3) $\left.\mathrm{E}\left[T_{i j}(\mathbf{0}) T_{k l}(\boldsymbol{x})\right]\right|_{i=k \neq j=l}$; that is, auto-correlations of off-diagonal terms

$$
\mathrm{E}\left[T_{12}(\mathbf{0}) T_{12}(\boldsymbol{x})\right]=K_{2}+\left(x_{1}^{2}+x_{2}^{2}\right) K_{4}+x_{1}^{2} x_{2}^{2} K_{5}
$$

and then $\mathrm{E}\left[T_{23}(\mathbf{0}) T_{23}(\boldsymbol{x})\right]$ and $\mathrm{E}\left[T_{31}(\mathbf{0}) T_{31}(\boldsymbol{x})\right]$ by cyclic permutations $1 \rightarrow$ $2 \rightarrow 3$.

(4) $\left.\mathrm{E}\left[T_{i j}(\mathbf{0}) T_{k l}(\boldsymbol{x})\right]\right|_{j \neq i=k \neq l \neq j}$; that is, cross-correlations of off-diagonal terms

$$
\mathrm{E}\left[T_{12}(\mathbf{0}) T_{13}(\boldsymbol{x})\right]=x_{2} x_{3} K_{4}+x_{1}^{2} x_{2} x_{3} K_{5}
$$

and then $\mathrm{E}\left[T_{13}(\mathbf{0}) T_{32}(\boldsymbol{x})\right]$ and $\mathrm{E}\left[T_{32}(\mathbf{0}) T_{12}(\boldsymbol{x})\right]$ by cyclic permutations $1 \rightarrow$ $2 \rightarrow 3$.

(5) $\left.\mathrm{E}\left[T_{i j}(\mathbf{0}) T_{k l}(\boldsymbol{x})\right]\right|_{i=j=k \neq l \neq j}$; that is, cross-correlations of diagonal terms with off-diagonal terms such as

$$
\mathrm{E}\left[T_{11}(\mathbf{0}) T_{12}(\boldsymbol{x})\right]=x_{1} x_{2}\left(K_{3}+2 K_{4}\right)+x_{1} x_{2}^{3} K_{5}
$$

and

$$
\mathrm{E}\left[T_{12}(\mathbf{0}) T_{13}(\boldsymbol{x})\right]=x_{2} x_{3} K_{3}+x_{1}^{2} x_{2} x_{3} K_{5}
$$

and the others by cyclic permutations $1 \rightarrow 2 \rightarrow 3$.

In principle, we can determine these five correlations for a specific physical situation. For example, when $\boldsymbol{T}$ is the antiplane elasticity tensor for a given resolution (or mesoscale) [Ostoja-Starzewski 2008], we can use micromechanics or experiments and then determine the best fits of the $K_{n}(n=1, \ldots, 5)$ coefficients.

\section{Concluding remarks}

Remark 4.1. On the one hand, Lomakin's functions (3-3) are simpler than functions (3-1). On the other hand, the restrictions of the functions (3-1) to the unit sphere $S^{2} \subset \mathbb{R}^{3}$ are orthogonal in the space of the square-integrable functions on $S^{2}$. Using this property, in a forthcoming paper we will obtain spectral expansions of tensor-valued homogeneous and isotropic random fields similar to those of Yaglom [1957].

Remark 4.2. The spherical harmonics $Y_{\ell m}(\theta, \varphi)$ are proportional to the Wigner $D$-functions by [Varshalovich et al. 1975, Equation (37), §5.2]:

$$
Y_{\ell m}(\theta, \varphi)=(-1)^{m} \sqrt{\frac{2 \ell+1}{4 \pi}} D_{-m 0}^{\ell}(0, \theta, \varphi) .
$$

The matrix entries $U_{\ell 0}^{i}\left(k_{x}\right)$ are proportional to the zonal, sectorial, and tesseral harmonics defined by [Varshalovich et al. 1975, Equation (14), §5.1]. 
Remark 4.3. The matrix entries $U_{j 0}^{i}\left(k_{x}\right)$, the Godunov-Gordienko coefficients, and (3-4) were calculated and proved by hand. Afterwards, they were checked using MATLAB and Symbolic Math Toolbox ${ }^{\mathrm{TM}}$ [Mathworks 2013]. The problem of an algebraic proof of the second and fourth equations in (3-4) remains open.

\section{Appendix: Proofs}

Proof of Lemma 2.2. The two-point correlation function $B(\boldsymbol{x})$ is a linear operator in $V$. It is known that the space of linear operators in $V$ is isomorphic to the tensor product $V^{*} \otimes V$, where $V^{*}$ is the set of all $\mathbb{R}$-linear maps $\boldsymbol{v}^{*}: V \rightarrow \mathbb{R}$. We need to prove that for any $S \in V^{*} \otimes V$ the following equality holds true:

$$
U(k) \mathrm{SU}^{-1}(k)=(U \otimes U)(k) S .
$$

Note that the set of tensors satisfying the above equality form a linear space. Therefore, it is enough to prove this equality for tensors of the form $\boldsymbol{v}^{*} \otimes \boldsymbol{v}$, where $\boldsymbol{v} \in V$ and $\boldsymbol{v}^{*} \in V^{*}$. The linear operator $\boldsymbol{v}^{*} \otimes \boldsymbol{v}$ acts on $V$ by

$$
\left(\boldsymbol{v}^{*} \otimes \boldsymbol{v}\right) \boldsymbol{w}=\boldsymbol{v}^{*}(\boldsymbol{w}) \boldsymbol{v}, \quad \boldsymbol{w} \in V .
$$

For this operator,

$$
\begin{aligned}
\left(U(k) \boldsymbol{v}^{*} \otimes \boldsymbol{v} U^{-1}(k)\right) \boldsymbol{w} & =U(k) \boldsymbol{v}^{*}\left(U^{-1}(k) \boldsymbol{w}\right) \boldsymbol{v}=\left(U(k) \boldsymbol{v}^{*}\right)(\boldsymbol{w}) U(k) \boldsymbol{v} \\
& =\left(U(k) \boldsymbol{v}^{*}\right) \otimes(U(k) \boldsymbol{v}) \boldsymbol{w}=(U \otimes U)(k)\left(\boldsymbol{v}^{*} \otimes \boldsymbol{v}\right) \boldsymbol{w} .
\end{aligned}
$$

By linearity, this equality follows for any $S \in V^{*} \otimes V$.

Proof of Theorem 3.1. The first equation in (1-1) may be written in the Gordienko basis as

$$
U^{1}(k) \boldsymbol{E}=\boldsymbol{E} .
$$

The representation $U^{1}$ is irreducible. By Lemma 2.1, $\boldsymbol{E}=\mathbf{0}$.

We cannot apply Lemma 2.1 to (2-11), because the representation $U^{1} \otimes U^{1}$ acting in $V^{1} \otimes V^{1}$ is reducible.

By definition, a tensor field $B(x)$ is a function on $\mathbb{R}^{3}$ with values in the linear space of real-valued bilinear forms on $V^{1} \times V^{1}$. The component $B_{i j}(x)$ is the value of the bilinear form $B(\boldsymbol{x})$ at the point $\left(\boldsymbol{h}_{i}^{1}, \boldsymbol{h}_{j}^{1}\right) \in V^{1} \times V^{1}$ :

$$
B_{i j}(\boldsymbol{x})=B\left(\boldsymbol{x} ; \boldsymbol{h}_{i}^{1}, \boldsymbol{h}_{j}^{1}\right) .
$$

The last equality may be rewritten as

$$
B_{i j}(\boldsymbol{x})=B\left(\boldsymbol{x} ; \boldsymbol{h}_{i}^{1} \otimes \boldsymbol{h}_{j}^{1}\right),
$$

where the right-hand side is the value of the linear form $B(\boldsymbol{x})$ at the point $\boldsymbol{h}_{i}^{1} \otimes \boldsymbol{h}_{j}^{1} \in$ $V^{1} \otimes V^{1}$. In other words, tensor products simplify multilinear forms to linear ones. 
Using (2-9) with $m=p=1, n=1$, and $q=j$, we obtain

$$
\begin{aligned}
B_{i j}(\boldsymbol{x}) & =B\left(\boldsymbol{x} ; \boldsymbol{h}_{i}^{1} \otimes \boldsymbol{h}_{j}^{1}\right)=B\left(\boldsymbol{x} ; \sum_{m=0}^{2} \sum_{\ell=-m}^{m} g_{m[1,1]}^{\ell[i, j]} \boldsymbol{h}_{\ell}^{m}\right) \\
& =\sum_{m=0}^{2} \sum_{\ell=-m}^{m} g_{m[1,1]}^{\ell[i, j]} B\left(\boldsymbol{x} ; \boldsymbol{h}_{\ell}^{m}\right) .
\end{aligned}
$$

Let $\boldsymbol{B}_{m}(\boldsymbol{x}) \in V^{m}$ be the vector field with components $B\left(\boldsymbol{x} ; \boldsymbol{h}_{\ell}^{m}\right),-m \leq \ell \leq m$. For this field, we have

$$
\boldsymbol{B}_{m}(k \boldsymbol{x})=U^{m}(k) \boldsymbol{B}_{m}(\boldsymbol{x}), \quad k \in \mathrm{SO}(3) .
$$

By Lemma 2.1, $\boldsymbol{B}_{m}(\mathbf{0})=\mathbf{0}$ for $m \geq 1$, while $\boldsymbol{B}_{0}(\mathbf{0})$ may take any real value.

Let $\mathrm{SO}(2)$ be the subgroup of rotations around $x_{0}$ axis. The restriction of the representation $U^{m}$ to $\mathrm{SO}(2)$ is the direct sum of the trivial representation $W^{0}$ of $\mathrm{SO}(2)$ acting in the one-dimensional space spanned by the vector $\boldsymbol{h}_{0}^{m}$ and of the irreducible representations

$$
W^{\ell}(\varphi)=\left(\begin{array}{cc}
\cos (\ell \varphi) & \sin (\ell \varphi) \\
-\sin (\ell \varphi) & \cos (\ell \varphi)
\end{array}\right), \quad \varphi \in \operatorname{SO}(2),
$$

acting in the two-dimensional spaces spanned by the vectors $\boldsymbol{h}_{\ell}^{m}$ and $\boldsymbol{h}_{-\ell}^{m}, 1 \leq \ell \leq m$. If $\boldsymbol{x}=(0,\|\boldsymbol{x}\|, 0)^{\top} \neq \mathbf{0}$, then $k \boldsymbol{x}=\boldsymbol{x}, k \in \mathrm{SO}(2)$, and

$$
\left(B\left(\boldsymbol{x} ; \boldsymbol{h}_{-\ell}^{m}\right), B\left(\boldsymbol{x} ; \boldsymbol{e}_{\ell}^{m}\right)\right)^{\top}=W^{\ell}(\varphi)\left(B\left(\boldsymbol{x} ; \boldsymbol{h}_{-\ell}^{m}\right), B\left(\boldsymbol{x} ; \boldsymbol{h}_{\ell}^{m}\right)\right)^{\top}, \quad 1 \leq \ell \leq m .
$$

By Lemma 2.1, $B\left(\boldsymbol{x} ; \boldsymbol{h}_{-\ell}^{m}\right)=B\left(\boldsymbol{x} ; \boldsymbol{h}_{\ell}^{m}\right)=0$, if $1 \leq \ell \leq m$, while $B\left(\boldsymbol{x} ; \boldsymbol{h}_{0}^{m}\right)$ is an arbitrary continuous real-valued function with $B\left(\mathbf{0} ; \boldsymbol{h}_{0}^{m}\right)=0$ for $m \geq 1$. In what follows, we denote this function by $B_{m}(\|x\|)$.

By (A.2),

$$
B\left(\boldsymbol{x} ; \boldsymbol{h}_{\ell}^{m}\right)=U_{\ell 0}^{m}\left(k_{\boldsymbol{x}}\right) B_{m}(\|\mathbf{x}\|) .
$$

Substitute (A.3) into (A.1). We obtain

$$
B_{i j}(\boldsymbol{x})=\sum_{m=0}^{2} \sum_{\ell=-m}^{m} g_{m[1,1]}^{\ell[i, j]} U_{\ell 0}^{m}\left(k_{\boldsymbol{x}}\right) B_{m}(\|\boldsymbol{x}\|) .
$$

In other words, $B_{i j}(\boldsymbol{x})$ is the sum of three matrix-valued functions

$$
B_{i j}(\boldsymbol{x})=\sum_{m=0}^{2} B_{i j}^{m}(\boldsymbol{x}),
$$

with

$$
B_{i j}^{m}(\boldsymbol{x})=\sum_{\ell=-m}^{m} g_{m[1,1]}^{\ell[i, j]} U_{\ell 0}^{m}\left(k_{\boldsymbol{x}}\right) B_{m}(\|\boldsymbol{x}\|) .
$$


Let $m=0$. Then

$$
U_{00}^{0}\left(k_{x}\right)=1,
$$

the matrix entry of the $1 \times 1$ identity matrix. To calculate the Godunov-Gordienko coefficients $g_{0[1,1]}^{0[i, j]}$, use the following property [Godunov and Gordienko 2004]:

$$
g_{N\left[N_{1}, N_{2}\right]}^{n\left[n_{1}, n_{2}\right]}=(-1)^{N+N_{1}+N_{2}} \sqrt{\frac{2 N+1}{2 N_{1}+1}} g_{N_{1}\left[N, N_{2}\right]}^{n_{1}\left[n, n_{2}\right]},
$$

with $N=n=0, N_{1}=N_{2}=1, n_{1}=i$, and $n_{2}=j$. We have

$$
g_{0[1,1]}^{0[i, j]}=\sqrt{1 / 3} g_{1[0,1]}^{i[0, j]} \text {. }
$$

The coefficients in the right-hand side can be calculated using (2-9):

$$
\boldsymbol{h}_{0}^{0} \otimes \boldsymbol{h}_{j}^{1}=\sum_{i=-1}^{1} g_{1[0,1]}^{i[0, j]} \boldsymbol{h}_{i}^{1}
$$

The left-hand side is clearly equal to $\boldsymbol{h}_{j}^{1}$. Therefore, $g_{1[0,1]}^{i[0, j]}=\delta_{i j}$,

$$
g_{0[1,1]}^{0[i, j]}=\sqrt{1 / 3} \delta_{i j}
$$

and the first matrix is

$$
B_{i j}^{0}(\boldsymbol{x})=\sqrt{1 / 3} \delta_{i j} B_{0}(\|\boldsymbol{x}\|) .
$$

Let $m=1$. Using (2-5)-(2-7), we obtain

$$
\left(\begin{array}{c}
U_{-10}^{1}(\varphi, \theta) \\
U_{00}^{1}(\varphi, \theta) \\
U_{10}^{1}(\varphi, \theta)
\end{array}\right)=\left(\begin{array}{ccc}
\cos \varphi & 0 & \sin \varphi \\
0 & 1 & 0 \\
-\sin \varphi & 0 & \cos \varphi
\end{array}\right)\left(\begin{array}{c}
0 \\
\cos \theta \\
\sin \theta
\end{array}\right)=\left(\begin{array}{c}
\sin \varphi \sin \theta \\
\cos \theta \\
\cos \varphi \sin \theta
\end{array}\right),
$$

or

$$
U_{\ell 0}^{1}\left(k_{x}\right)=\frac{x_{\ell}}{r} .
$$

The Godunov-Gordienko coefficients $g_{1[1,1]}^{\ell[i, j]}$ are calculated by [Godunov and Gordienko 2004, Formulae (1.26)-(1.29)]. The nonzero coefficients are

$$
\begin{aligned}
& g_{1[1,1]}^{-1[1,0]}=g_{1[1,1]}^{0[-1,1]}=g_{1[1,1]}^{1[0,-1]}=\sqrt{1 / 2}, \\
& g_{1[1,1]}^{-1[0,1]}=g_{1[1,1]}^{0[1,-1]}=g_{1[1,1]}^{1[-1,0]}=-\sqrt{1 / 2} .
\end{aligned}
$$

The second matrix becomes

$$
\frac{1}{r \sqrt{2}}\left(\begin{array}{ccc}
0 & -x_{1} & x_{0} \\
x_{1} & 0 & -x_{-1} \\
-x_{0} & x_{-1} & 0
\end{array}\right) B_{1}(\|\boldsymbol{x}\|) .
$$


This matrix is not symmetric but skew-symmetric. The matrix $B_{i j}(\boldsymbol{x})$ is symmetric if and only if $B_{1}(\|x\|)=0$.

The matrix entries $U_{\ell 0}^{2}\left(k_{x}\right)$ can be calculated using (2-5)-(2-7) as

$$
\begin{aligned}
U_{-20}^{2}\left(k_{x}\right) & =-\frac{\sqrt{3} x_{-1} x_{1}}{r^{2}}, & U_{-10}^{2}\left(k_{x}\right) & =\frac{\sqrt{3} x_{-1} x_{0}}{r^{2}}, \quad U_{00}^{2}\left(k_{x}\right)=\frac{3 x_{0}^{2}}{2 r^{2}}-\frac{1}{2}, \\
U_{10}^{2}\left(k_{x}\right) & =\frac{\sqrt{3} x_{0} x_{1}}{r^{2}}, & U_{20}^{2}\left(k_{x}\right) & =\frac{\sqrt{3}\left(x_{-1}^{2}-x_{1}^{2}\right)}{2 r^{2}} .
\end{aligned}
$$

To calculate the Godunov-Gordienko coefficients $g_{2[1,1]}^{\ell[i, j]}$, use (A.5) with $N=2$, $N_{1}=N_{2}=1, n=\ell, n_{1}=i$, and $n_{2}=j$. We have

$$
g_{2[1,1]}^{\ell[i, j]}=\sqrt{5 / 3} g_{1[2,1]}^{i[\ell, j]} .
$$

The coefficients $g_{1[2,1]}^{i[\ell, j]}$ are calculated by [Godunov and Gordienko 2004, Formulae (1.30)-(1.34)]. The nonzero Godunov-Gordienko coefficients are

$$
\begin{aligned}
g_{2[1,1]}^{-2[-1,1]} & =g_{2[1,1]}^{-2[1,-1]}=g_{2[1,1]}^{2[1,1]}=-\sqrt{1 / 2}, \\
g_{2[1,1]}^{0[-1,-1]} & =g_{2[1,1]}^{0[1,1]}=-\sqrt{1 / 6}, \\
g_{2[1,1]}^{-1[-1,0]} & =g_{2[1,1]}^{-1[0,-1]}=g_{2[1,1]}^{1[0,1]}=g_{2[1,1]}^{1[1,0]}=g_{2[1,1]}^{2[-1,-1]}=\sqrt{1 / 2}, \\
g_{2[1,1]}^{0[0,0]} & =\sqrt{2 / 3} .
\end{aligned}
$$

In (2-10) put $k=j=0, m=p=1, n=i$, and $q=j$. We have

$$
U_{i 0}^{1}(k) U_{j 0}^{1}(k)=\sum_{s=0}^{2} \sum_{t=-s}^{s} \sum_{\ell=-s}^{s} g_{s[1,1]}^{t[i, j]} U_{t \ell}^{s}(k) g_{s[1,1]}^{\ell[0,0]} .
$$

Using the above-calculated values of the matrix entries and Godunov-Gordienko coefficients, we may rewrite this formula as

$$
\frac{x_{i} x_{j}}{r^{2}}=\frac{1}{3} \delta_{i j}+\sqrt{\frac{2}{3}} \sum_{t=-2}^{2} g_{2[1,1]}^{t[i, j]} U_{t 0}^{2}\left(k_{x}\right)
$$

or

$$
\sum_{t=-2}^{2} g_{2[1,1]}^{t[i, j]} U_{t 0}^{2}\left(k_{x}\right)=\frac{\sqrt{3}}{\sqrt{2} r^{2}}\left(x_{j} x_{j}-\frac{r^{2}}{3} \delta_{i j}\right) .
$$

We introduce the following notation:

$$
K_{0}(\|x\|)=\frac{1}{\sqrt{3}} B_{0}(\|x\|)-\frac{1}{\sqrt{6}} B_{2}(\|x\|), \quad K_{2}(\|x\|)=\frac{\sqrt{3}}{\sqrt{2} r^{2}} B_{2}(\|x\|) .
$$

The theorem is proved. Note that the space $V^{0}$ consists of tensors proportional to the Kronecker delta, $V^{1}$ consists of skew-symmetric tensors, and $V^{2}$ consists of symmetric traceless tensors. 
Proof of Theorem 3.2. Use Lemma 2.2 to write the representation $U$ as $U=$ $U^{1} \otimes U^{1}$. The first equation in (1-1) takes the form

$$
E(k \boldsymbol{x})=\left(U^{1} \otimes U^{1}\right)(k) E(\boldsymbol{x}) .
$$

or

$$
E=\left(U^{1} \otimes U^{1}\right)(k) E, \quad k \in \mathrm{SO}(3) .
$$

In other words, $E$ lies in the subspace of $V$ where the trivial representation is realized, or

$$
\mathrm{E}\left[T_{i j}(\boldsymbol{x})\right]=C \delta_{i j}, \quad C \in \mathbb{R} .
$$

The second equation in (1-1) takes the form

$$
B(k \boldsymbol{x})=\left(U^{1} \otimes U^{1} \otimes U^{1} \otimes U^{1}\right)(k) B(\boldsymbol{x}) .
$$

We write $B(\boldsymbol{x})$ as a bilinear form on pairs of tensors, $\left(V^{1} \otimes V^{1}\right) \times\left(V^{1} \otimes V^{1}\right)$ :

$$
B^{i j k \ell}(\boldsymbol{x})=B\left(\boldsymbol{x} ; \boldsymbol{h}_{i}^{1} \otimes \boldsymbol{h}_{j}^{1}, \boldsymbol{h}_{\ell}^{1} \otimes \boldsymbol{h}_{m}^{1}\right) .
$$

Note that the tensor field $B^{i j \ell m}(\boldsymbol{x})$ is symmetric in the following sense:

$$
B^{\ell m i j}(\boldsymbol{x})=B^{i j \ell m}(\boldsymbol{x}) .
$$

Recall that $V^{1} \otimes V^{1}=V^{0} \oplus V^{1} \oplus V^{2}$. Rewrite $B(x)$ as a bilinear form on $\left(V^{0} \oplus V^{1} \oplus V^{2}\right) \times\left(V^{0} \oplus V^{1} \oplus V^{2}\right)$ :

$$
B^{i j \ell m}(\boldsymbol{x})=B\left(\boldsymbol{x} ; \sum_{n=0}^{2} \sum_{p=-n}^{n} g_{n[1,1]}^{p[i, j]} \boldsymbol{h}_{p}^{n}, \sum_{q=0}^{2} \sum_{r=-q}^{q} g_{q[1,1]}^{r[\ell, m]} \boldsymbol{h}_{r}^{q}\right) .
$$

In fact, $B(\boldsymbol{x})$ is a bilinear form on pairs of symmetric tensors, which is to say, on $\left(V^{0} \oplus V^{2}\right) \times\left(V^{0} \oplus V^{2}\right)$ :

$$
\begin{aligned}
B^{i j \ell m}(\boldsymbol{x}) & =B\left(\boldsymbol{x} ; \sum_{n \in\{0,2\}} \sum_{p=-n}^{n} g_{n[1,1]}^{p[i, j]} \boldsymbol{h}_{p}^{n}, \sum_{q \in\{0,2\}} \sum_{r=-q}^{q} g_{q[1,1]}^{r[\ell, m]} \boldsymbol{h}_{r}^{q}\right) \\
& =\sum_{(n, q) \in\{0,2\} \times\{0,2\}} \sum_{p=-n}^{n} \sum_{r=-q}^{q} g_{n[1,1]}^{p[i, j]} g_{q[1,1]}^{r[\ell, m]} B\left(\boldsymbol{x} ; \boldsymbol{h}_{p}^{n}, \boldsymbol{h}_{r}^{q}\right) \\
& =\sum_{(n, q) \in\{0,2\} \times\{0,2\}} \sum_{p=-n}^{n} \sum_{r=-q}^{q} g_{n[1,1]}^{p[i, j]} g_{q[1,1]}^{r[\ell, m]} B\left(\boldsymbol{x} ; \boldsymbol{h}_{p}^{n} \otimes \boldsymbol{h}_{r}^{q}\right) \\
& =\sum_{(n, q) \in\{0,2\} \times\{0,2\}} \sum_{p=-n}^{n} \sum_{r=-q}^{q} g_{n[1,1]}^{p[i, j]} g_{q[1,1]}^{r[\ell, m]} B\left(\boldsymbol{x} ; \sum_{s=|n-q|}^{n+q} \sum_{t=-s}^{s} g_{s[m, p]}^{t[n, q]} \boldsymbol{h}_{t}^{s}\right) .
\end{aligned}
$$

The possible values for $s$ are $0 \leq s \leq 4$. Let $A_{s}$ be the set of all pairs $(n, q) \in$ $\{0,2\} \times\{0,2\}$ such that $V^{s} \subseteq V^{n} \otimes V^{q}$. We have 
$A_{0}=\{(0,0),(2,2)\}, \quad A_{1}=A_{3}=A_{4}=\{(2,2)\}, \quad A_{2}=\{(0,2),(2,0),(2,2)\}$,

and

$$
B^{i j \ell m}(\boldsymbol{x})=\sum_{s=0}^{4} B_{s}^{i j \ell m}(\boldsymbol{x})
$$

where

$$
B_{s}^{i j \ell m}(\boldsymbol{x})=\sum_{(n, q) \in A_{s}} \sum_{p=-n}^{n} \sum_{r=-q}^{q} g_{n[1,1]}^{p[i, j]} g_{q[1,1]}^{r[\ell, m]} \sum_{t=-s}^{s} g_{s[m, p]}^{t[n, q]} B\left(\boldsymbol{x} ; \boldsymbol{h}_{t}^{s}(n, q)\right),
$$

and where the pairs $(n, q) \in A_{s}$ enumerate copies of the vector $\boldsymbol{h}_{t}^{s}$. By (A.3),

$$
B\left(\boldsymbol{x} ; \boldsymbol{h}_{t}^{s}(n, q)\right)=U_{t 0}^{s}\left(k_{x}\right) B_{s(n, q)}(\|\boldsymbol{x}\|),
$$

where $B_{s(n, q)}(\|\boldsymbol{x}\|)$ are continuous real-valued functions with $B_{s(n, q)}(0)=0$ for $s \geq 1$. We obtain

$$
B_{s}^{i j \ell m}(\boldsymbol{x})=\sum_{(n, q) \in A_{s}} C_{n q s}^{i j \ell m}(\boldsymbol{x})
$$

where

$$
C_{n q s}^{i j \ell m}(\boldsymbol{x})=\sum_{p=-n}^{n} \sum_{r=-q}^{q} g_{n[1,1]}^{p[i, j]} g_{q[1,1]}^{r[\ell, m]} \sum_{t=-s}^{s} g_{s[n, q]}^{t[p, r]} U_{t 0}^{s}\left(k_{\boldsymbol{x}}\right) B_{s(n, q)}(\|\boldsymbol{x}\|) .
$$

We calculate the functions $C_{n q s}^{i j \ell m}(\boldsymbol{x})$.

Let $(n, q)=(0,0) \in A_{0}$. The corresponding term is

$$
C_{000}^{i j \ell m}(\boldsymbol{x})=\frac{1}{3} \delta_{i j} \delta_{\ell m} B_{0(0,0)}(\|\boldsymbol{x}\|)=M_{i j \ell m}^{1}(\boldsymbol{x}) K_{1}(\|\boldsymbol{x}\|),
$$

by (A.4) and (A.6) with $K_{1}(\|\boldsymbol{x}\|)=B_{0(0,0)}(\|\boldsymbol{x}\|)$.

Let $(n, q)=(2,2) \in A_{0}$. The corresponding term is

$$
C_{220}^{i j \ell m}(\boldsymbol{x})=\sum_{r=-2}^{2} \sum_{s=-2}^{2} g_{2[1,1]}^{r[i, j]} g_{2[1,1]}^{s[\ell, m]} g_{0[2,2]}^{0[r, s]} U_{00}^{0}\left(k_{\boldsymbol{x}}\right) B_{0(2,2)}(\|\boldsymbol{x}\|) .
$$

The Godunov-Gordienko coefficients $g_{2[1,1]}^{r[i, j]}$ and $g_{2[1,1]}^{s[\ell, m]}$ are calculated by (A.6), while the coefficients $g_{0[2,2]}^{0[n, q]}$ are calculated using (A.5) with $n=N=0, n_{1}=r$, $n_{2}=s, N_{1}=N_{2}=2$ and (2-9):

$$
g_{0[2,2]}^{0[r, s]}=\sqrt{1 / 5} g_{2[0,2]}^{r[0, s]}=\sqrt{1 / 5} \delta_{r s} .
$$

Therefore, we obtain

$$
C_{220}^{i j \ell m}(\boldsymbol{x})=\sqrt{1 / 5} \sum_{r=-2}^{2} g_{2[1,1]}^{r[i, j]} g_{2[1,1]}^{r[\ell, m]} B_{0(2,2)}(\|x\|)=M_{i j \ell m}^{2}(\boldsymbol{x}) K_{2}(\|\boldsymbol{x}\|)
$$

with $K_{2}(\|\boldsymbol{x}\|)=B_{0(2,2)}(\|\boldsymbol{x}\|)$. 
Let $(n, q)=(2,2) \in A_{1}$. The corresponding term is

$$
C_{221}^{i j \ell m}(\boldsymbol{x})=\sum_{p=-2}^{2} \sum_{s=-2}^{2} g_{2[1,1]}^{p[i, j]} g_{2[1,1]}^{r[\ell, m]} \sum_{s=-1}^{1} g_{1[2,2]}^{s[p, r]} U_{s 0}^{1}\left(k_{\boldsymbol{x}}\right) B_{1(2,2)}(\|\boldsymbol{x}\|) .
$$

The Godunov-Gordienko coefficients $g_{1[2,2]}^{s[p, r]}$ are calculated by [Godunov and Gordienko 2004, Formulae (1.26)-(1.29)]. The nonzero elements of the GodunovGordienko matrix $G_{1[2,2]}^{-1}$ are

$$
\begin{array}{ll}
g_{1[2,2]}^{-1[0,1]}=-\sqrt{3 / 10}, & g_{1[2,2]}^{-1[-2,-1]}=g_{1[2,2]}^{-1[2,1]}=-\sqrt{1 / 10}, \\
g_{1[2,2]}^{-1[1,0]}=\sqrt{3 / 10}, & g_{1[2,2]}^{-1[-1,-2]}=g_{1[2,2]}^{-1[1,2]}=\sqrt{1 / 10},
\end{array}
$$

and those of the matrix $G_{1[2,2]}^{0}$ are

$$
\begin{array}{ll}
g_{1[2,2]}^{0[2,-2]}=-\sqrt{2 / 5}, & g_{1[2,2]}^{0[1,-1]}=-\sqrt{1 / 10}, \\
g_{1[2,2]}^{0[-2,2]}=\sqrt{2 / 5}, & g_{1[2,2]}^{0[-1,1]}=\sqrt{1 / 10} .
\end{array}
$$

Finally, the nonzero elements of the Godunov-Gordienko matrix $G_{1[2,2]}^{-1}$ are

$$
\begin{array}{ll}
g_{1[2,2]}^{1[0,-1]}=\sqrt{3 / 10}, & g_{1[2,2]}^{1[1,-2]}=g_{1[2,2]}^{1[2,-1]}=-\sqrt{1 / 10}, \\
g_{1[2,2]}^{1[-1,0]}=-\sqrt{3 / 10}, & g_{1[2,2]}^{1[-2,1]}=g_{1[2,2]}^{1[-1,2]}=\sqrt{1 / 10} .
\end{array}
$$

We see that the Godunov-Gordienko matrices $g_{1[2,2]}^{s},-1 \leq s \leq 1$, are skewsymmetric. We have $B_{1(2,2)}(\|\boldsymbol{x}\|)=0$ by the same reasons as $B_{1}(\|\boldsymbol{x}\|)=0$ in the proof of Theorem 3.1.

Let $(n, q)=(0,2) \in A_{2}$. The corresponding term is

$$
C_{022}^{i j \ell m}(\boldsymbol{x})=g_{0[1,1]}^{0[i, j]} \sum_{p=-2}^{2} g_{2[1,1]}^{p[\ell, m]} \sum_{s=-2}^{2} g_{2[0,2]}^{s[0, p]} U_{s 0}^{2}\left(k_{\boldsymbol{x}}\right) B_{2(0,2)}(\|\boldsymbol{x}\|) .
$$

Using (A.6), we obtain

$$
C_{022}^{i j \ell m}(\boldsymbol{x})=\sqrt{1 / 3} \delta_{i j} \sum_{p=-2}^{2} g_{2[1,1]}^{p[\ell, m]} \sum_{s=-2}^{2} g_{2[0,2]}^{s[0, r]} U_{s 0}^{2}\left(k_{\boldsymbol{x}}\right) B_{2(0,2)}(\|\boldsymbol{x}\|) .
$$

Clearly $g_{2[0,2]}^{s[0, p]}=\delta_{p s}$. Therefore,

$$
C_{022}^{i j \ell m}(\boldsymbol{x})=\sqrt{1 / 3} \delta_{i j} \sum_{p=-2}^{2} g_{2[1,1]}^{p[\ell, m]} U_{p 0}^{2}\left(k_{x}\right) B_{2(0,2)}(\|\boldsymbol{x}\|) .
$$

Let $(n, q)=(2,0) \in A_{2}$. The corresponding term is

$$
C_{202}^{i j \ell m}(\boldsymbol{x})=g_{0[1,1]}^{0[\ell, m]} \sum_{p=-2}^{2} g_{2[1,1]}^{p[i, j]} \sum_{s=-2}^{2} g_{2[2,0]}^{t[p, 0]} U_{s 0}^{2}\left(k_{\boldsymbol{x}}\right) B_{2(2,0)}(\|\boldsymbol{x}\|) .
$$


Using (A.6), we obtain

$$
C_{202}^{i j \ell m}(\boldsymbol{x})=\sqrt{1 / 3} \delta_{\ell m} \sum_{p=-2}^{2} g_{2[1,1]}^{p[i, j]} \sum_{s=-2}^{2} g_{2[2,0]}^{t[p, 0]} U_{s 0}^{2}\left(k_{x}\right) B_{2(2,0)}(\|\boldsymbol{x}\|) .
$$

Clearly $g_{2[2,0]}^{s[p, 0]}=\delta_{p s}$. Therefore,

$$
C_{202}^{i j \ell m}(\boldsymbol{x})=\sqrt{1 / 3} \delta_{\ell m} \sum_{p=-2}^{2} g_{2[1,1]}^{p[i, j]} U_{p 0}^{2}\left(k_{\boldsymbol{x}}\right) B_{2(2,0)}(\|\boldsymbol{x}\|) .
$$

We see that the tensor field $B^{i j \ell m}(x)$ is symmetric if and only if $B_{2(0,2)}(\|x\|)=$ $B_{2(2,0)}(\|\boldsymbol{x}\|)$. Denote their common value by $K_{3}(\|\boldsymbol{x}\|)$ and put

$$
C_{2}^{i j \ell m}(\boldsymbol{x})=\frac{1}{\sqrt{2}}\left(C_{022}^{i j \ell m}(\boldsymbol{x})+C_{202}^{i j \ell m}(\boldsymbol{x})\right) .
$$

We obtain

$$
C_{2}^{i j \ell m}(\boldsymbol{x})=M_{i j \ell m}^{3}(\boldsymbol{x}) K_{3}(\|\boldsymbol{x}\|) .
$$

Let $(n, q)=(2,2) \in A_{2}$. The corresponding term is

$$
\begin{aligned}
C_{222}^{i j \ell m}(\boldsymbol{x}) & =\sum_{p=-2}^{2} \sum_{r=-2}^{2} g_{2[1,1]}^{p[i, j]} g_{2[1,1]}^{r[\ell, m]} \sum_{s=-2}^{2} g_{2[2,2]}^{s[p, r]} U_{s 0}^{2}\left(k_{x}\right) B_{2(2,2)}(\|\boldsymbol{x}\|) \\
& =M_{i j \ell m}^{4}(\boldsymbol{x}) K_{4}(\|\boldsymbol{x}\|),
\end{aligned}
$$

with $K_{4}(\|\boldsymbol{x}\|)=B_{2(2,2)}(\|\boldsymbol{x}\|)$.

To calculate Godunov-Gordienko coefficients $g_{2[2,2]}^{s[p, r]}$, we use generating function from [Godunov and Gordienko 2004, Formula (2.6)]. The nonzero coefficients are:

$$
\begin{aligned}
g_{2[2,2]}^{-2[0,-2]} & =g_{2[2,2]}^{-2[-2,0]}=g_{2[2,2]}^{0[-2,-2]}=g_{2[2,2]}^{0[2,2]}=g_{2[2,2]}^{2[2,0]}=g_{2[2,2]}^{2[0,2]}=-\sqrt{2 / 7}, \\
g_{2[2,2]}^{-2[1,-1]} & =g_{2[2,2]}^{-2[-1,1]}=g_{2[2,2]}^{-1[1,-2]}=g_{2[2,2]}^{-1[-2,1]}=g_{2[2,2]}^{1[-1,-2]}=g_{2[2,2]}^{1[-2,-1]}=g_{2[2,2]}^{1[2,1]} \\
& =g_{2[2,2]}^{1[1,2]}=g_{2[2,2]}^{2[1,1]}=-\sqrt{3 / 14}, \\
g_{2[2,2]}^{-1[0,-1]} & =g_{2[2,2]}^{-1[-1,0]}=g_{2[2,2]}^{0[-1,-1]}=g_{2[2,2]}^{0[1,1]}=g_{2[2,2]}^{1[0,1]}=g_{2[2,2]}^{1[1,0]}=\sqrt{1 / 14}, \\
g_{2[2,2]}^{-1[2,-1]} & =g_{2[2,2]}^{-1[-1,2]}=g_{2[2,2]}^{2[-1,-1]}=\sqrt{3 / 14}, \\
g_{2[2,2]}^{0[0,0]} & =\sqrt{2 / 7} .
\end{aligned}
$$

Let $(n, q)=(2,2) \in A_{3}$. The corresponding term is

$$
C_{223}^{i j \ell m}(\boldsymbol{x})=\sum_{r=-2}^{2} \sum_{s=-2}^{2} g_{2[1,1]}^{r[i, j]} g_{2[1,1]}^{s[\ell, m]} \sum_{t=-3}^{3} g_{3[2,2]}^{t[r, s]} U_{t 0}^{3}\left(k_{x}\right) B_{3(2,2)}(\|\boldsymbol{x}\|) .
$$


The nonzero elements of the Godunov-Gordienko matrix $g_{3[2,2]}^{-3}$ are

$$
g_{3[2,2]}^{-3[-1,-2]}=g_{3[2,2]}^{-3[2,1]}=-1 / 2, \quad g_{3[2,2]}^{-3[-2,-1]}=g_{3[2,2]}^{-3[1,2]}=1 / 2,
$$

those of the matrix $g_{3[2,2]}^{-2}$ are

$$
g_{3[2,2]}^{-2[2,0]}=-\sqrt{1 / 2}, \quad g_{3[2,2]}^{-2[0,2]}=\sqrt{1 / 2},
$$

those of the matrix $g_{3[2,2]}^{-1}$ are

$$
\begin{aligned}
g_{3[2,2]}^{-1[0,1]} & =-\sqrt{1 / 5}, & g_{3[2,2]}^{-1[1,0]} & =\sqrt{1 / 5}, \\
g_{3[2,2]}^{-1[-1,-2]} & =g_{3[2,2]}^{-1[1,2]}=-\sqrt{3 / 20}, & g_{3[2,2]}^{-1[-2,-1]} & =g_{3[2,2]}^{-1[2,1]}=\sqrt{3 / 20},
\end{aligned}
$$

those of the matrix $g_{3[2,2]}^{0}$ are

$$
\begin{array}{ll}
g_{3[2,2]}^{0[1,-1]}=-\sqrt{2 / 5}, & g_{3[2,2]}^{0[-1,1]}=\sqrt{2 / 5}, \\
g_{3[2,2]}^{0[-2,2]}=-\sqrt{1 / 10}, & g_{3[2,2]}^{0[2,-2]}=\sqrt{1 / 10}
\end{array}
$$

those of the matrix $g_{3[2,2]}^{1}$ are

$$
\begin{array}{ll}
g_{3[2,2]}^{1[-1,0]}=-\sqrt{1 / 5}, & g_{3[2,2]}^{1[0,1]}=\sqrt{1 / 5}, \\
g_{3[2,2]}^{1[-2,1]}=g_{3[2,2]}^{1[-1,2]}=-\sqrt{3 / 20}, & g_{3[2,2]}^{1[1,-2]}=g_{3[2,2]}^{1[2,-11]}=\sqrt{3 / 20},
\end{array}
$$

and those of the matrix $g_{3[2,2]}^{2}$ are

$$
g_{3[2,2]}^{2[-2,0]}=-\sqrt{1 / 2}, \quad g_{3[2,2]}^{2[0,-22]}=\sqrt{1 / 2} .
$$

Finally, the nonzero elements of the Godunov-Gordienko matrix $g_{3[2,2]}^{3}$ are

$$
g_{3[2,2]}^{3[-1,2]}=g_{3[2,2]}^{3[1,-2]}=-1 / 2, \quad g_{3[2,2]}^{3[-2,1]}=g_{3[2,2]}^{3[2,-1]}=1 / 2 .
$$

We see that the Godunov-Gordienko matrices $g_{3[2,2]}^{t},-3 \leq t \leq 3$, are skewsymmetric. We have $B_{2(2,2)}(\|\boldsymbol{x}\|)=0$ by the same reasons as before.

Let $(n, q)=(2,2) \in A_{4}$. The corresponding term is

$$
\begin{aligned}
C_{224}^{i j \ell m}(\boldsymbol{x}) & =\sum_{r=-2}^{2} \sum_{s=-2}^{2} g_{2[1,1]}^{r[i, j]} g_{2[1,1]}^{s[\ell, m]} \sum_{t=-4}^{4} g_{4[2,2]}^{t[r, s]} U_{t 0}^{4}\left(k_{x}\right) B_{4(2,2)}(\|\boldsymbol{x}\|) \\
& =M_{i j \ell m}^{5}(\boldsymbol{x}) K_{5}(\|\boldsymbol{x}\|)
\end{aligned}
$$

with $K_{5}(\|\boldsymbol{x}\|)=B_{4(2,2)}(\|\boldsymbol{x}\|)$.

The nonzero elements of the Godunov-Gordienko matrices $g_{4[2,2]}^{ \pm 4}$ are

$$
g_{4[2,2]}^{-4[-2,2]}=g_{4[2,2]}^{-4[2,-2]}=g_{4[2,2]}^{4[2,2]}=-\sqrt{1 / 2}, \quad g_{4[2,2]}^{4[-2,-2]}=\sqrt{1 / 2},
$$


those of the matrices $g_{4[2,2]}^{ \pm 3}$ are

$$
\begin{aligned}
& g_{4[2,2]}^{-3[-2,1]}=g_{4[2,2]}^{-3[1,-2]}=g_{4[2,2]}^{-3[-1,2]}=g_{4[2,2]}^{-3[2,-1]}=g_{4[2,2]}^{3[1,2]}=g_{4[2,2]}^{3[2,1]}=-1 / 2, \\
& g_{4[2,2]}^{3[-2,-1]}=g_{4[2,2]}^{3[-1,-2]}=1 / 2,
\end{aligned}
$$

those of the matrices $g_{4[2,2]}^{ \pm 2}$ are

$$
\begin{aligned}
& g_{4[2,2]}^{-2[-1,1]}=g_{4[2,2]}^{-2[1,-1]}=g_{4[2,2]}^{2[1,1]}=-\sqrt{2 / 7}, \\
& g_{4[2,2]}^{-2[-2,0]}=g_{4[2,2]}^{-2[0,-2]}=g_{4[2,2]}^{2[0,2]}=g_{4[2,2]}^{2[2,0]}=\sqrt{3 / 14}, \\
& g_{4[2,2]}^{2[-1,-1]}=\sqrt{2 / 7},
\end{aligned}
$$

and those of the matrices $g_{4[2,2]}^{ \pm 1}$ are

$$
\begin{aligned}
& g_{4[2,2]}^{-1[-1,2]}=g_{4[2,2]}^{-1[2,-1]}=-1 /(2 \sqrt{7}), \\
& g_{4[2,2]}^{-1[-2,1]}=g_{4[2,2]}^{-1[1,-2]}=g_{4[2,2]}^{1[-2,-1]}=g_{4[2,2]}^{1[-1,-2]}=g_{4[2,2]}^{1[1,2]}=g_{4[2,2]}^{1[2,1]}=1 /(2 \sqrt{7}), \\
& g_{4[2,2]}^{-1[-1,0]}=g_{4[2,2]}^{-1[0,-1]}=g_{4[2,2]}^{1[0,1]}=g_{4[2,2]}^{1[1,0]}=\sqrt{3 / 7} .
\end{aligned}
$$

Finally, the nonzero elements of the Godunov-Gordienko matrix $g_{4[2,2]}^{0}$ are

$$
\begin{aligned}
g_{4[2,2]}^{0[-1,-1]} & =g_{4[2,2]}^{0[1,1]}=-\sqrt{8 / 35}, \\
g_{4[2,2]}^{0[-2,-2]} & =g_{4[2,2]}^{0[2,2]}=\sqrt{1 / 70}, \\
g_{4[2,2]}^{0[0,0]} & =6 / \sqrt{70} .
\end{aligned}
$$

Proof of (3-4). The first equation is obvious. The second and fourth equations may be checked by brute force, using the values of the matrix entries and GodunovGordienko coefficients calculated above. To prove the third equation, substitute (A.7) into (3-1). For the fifth equation, put $i=j=0$ and $m=p=2$ in (2-10). We have

$$
U_{r 0}^{2}\left(k_{\boldsymbol{x}}\right) U_{s 0}^{2}\left(k_{\boldsymbol{x}}\right)=\sum_{t=0}^{4} \sum_{v=-t}^{t} \sum_{w=-t}^{t} g_{t[2,2]}^{v[r, s]} U_{v w}^{t}\left(k_{\boldsymbol{x}}\right) g_{t[2,2]}^{w[0,0]}
$$

Using the matrix entries calculated above and the Godunov-Gordienko coefficients, we may rewrite this formula as

$$
U_{r 0}^{2}\left(k_{x}\right) U_{s 0}^{2}\left(k_{x}\right)=\frac{1}{5} \delta_{r s}+\sqrt{2 / 7} \sum_{t=-2}^{2} g_{2[2,2]}^{t[r, s]} U_{t 0}^{2}\left(k_{x}\right)+\frac{3 \sqrt{2}}{\sqrt{35}} \sum_{t=-4}^{4} g_{4[2,2]}^{t[r, s]} U_{t 0}^{4}\left(k_{x}\right) .
$$


Move all terms but the last from the right-hand side to the left-hand side, and substitute the result into the fifth line in (3-1). We obtain

$$
\begin{aligned}
M_{i j \ell m}^{5}(\boldsymbol{x})=\frac{\sqrt{35}}{3 \sqrt{2}} \sum_{n=-2}^{2} \sum_{p=-2}^{2} g_{2[1,1]}^{n[i, j]} g_{2[1,1]}^{p[\ell, m]}\left(U_{r 0}^{2}\left(k_{\boldsymbol{x}}\right) U_{s 0}^{2}\left(k_{\boldsymbol{x}}\right)-\frac{1}{5} \delta_{n p}\right. \\
\left.-\sqrt{2 / 7} \sum_{t=-2}^{2} g_{2[2,2]}^{t[r, s]} U_{t 0}^{2}\left(k_{\boldsymbol{x}}\right)\right) .
\end{aligned}
$$

Using (A.7) and (3-1), we get

$$
\begin{aligned}
M_{i j \ell m}^{5}(\boldsymbol{x})=\frac{\sqrt{35}}{3 \sqrt{2}}\left(\frac{3}{2} L_{i j \ell m}^{5}(\boldsymbol{x})-\frac{1}{2} L_{i j \ell m}^{4}(\boldsymbol{x})+\frac{1}{6} L_{i j \ell m}^{1}(\boldsymbol{x})\right) & \\
& -\frac{\sqrt{7}}{3 \sqrt{2}} M_{i j \ell m}^{2}(\boldsymbol{x})-\frac{\sqrt{5}}{3} M_{i j \ell m}^{4}(\boldsymbol{x}) .
\end{aligned}
$$

We finish the proof by using the second and fourth equations.

\section{References}

[Adams 1969] J. F. Adams, Lectures on Lie groups, W. A. Benjamin, New York, 1969.

[Boehler et al. 1994] J.-P. Boehler, A. A. Kirillov, Jr., and E. T. Onat, "On the polynomial invariants of the elasticity tensor", J. Elasticity 34:2 (1994), 97-110.

[Christakos 2005] G. Christakos, Random field models in earth sciences, Dover, Mineola, NY, 2005.

[Cressie 1993] N. A. C. Cressie, Statistics for spatial data, Wiley, New York, 1993.

[Godunov and Gordienko 2004] S. K. Godunov and V. M. Gordienko, “Коэффициенты КлебшаГордана при различных выборах базисов унитарных и ортогональных представлений групп SU(2), SO(3)", Sibirsk. Mat. Zh. 45:3 (2004), 540-557. Translated as "The ClebschGordan coefficients with respect to various bases for unitary and orthogonal representations of the groups SU(2) and SO(3)" in Sib. Math. J. 45:3 (2004), 443-458.

[Gordienko 2002] V. M. Gordienko, “Матричные элементы вещественных представлений групп $O(3)$ и SO(3)", Sibirsk. Mat. Zh. 43:1 (2002), 51-63. Translated as "Matrix entries of real representations of the groups $O(3)$ and $\mathrm{SO}(3)$ " in Sib. Math. J. 43:1 (2002), 36-46.

[Leonenko and Sakhno 2012] N. Leonenko and L. Sakhno, "On spectral representations of tensor random fields on the sphere”, Stoch. Anal. Appl. 30:1 (2012), 44-66.

[Lomakin 1964] V. A. Lomakin, "Statistical description of the stressed state of a body under deformation”, Dokl. Akad. Nauk SSSR 155:6 (1964), 1274-1277. In Russian.

[Malyarenko 2013] A. Malyarenko, Invariant random fields on spaces with a group action, Springer, Heidelberg, 2013.

[Marinucci and Peccati 2011] D. Marinucci and G. Peccati, Random fields on the sphere: representation, limit theorems and cosmological applications, London Mathematical Society Lecture Note Series 389, Cambridge University Press, 2011.

[Mathworks 2013] Symbolic math toolbox: user's guide, The Mathworks, Natick, MA, 2013, Available at http://www.mathworks.com/help/pdf_doc/symbolic/symbolic_tb.pdf. 
[Monin and Yaglom 1965] A. S. Monin and A. M. Yaglom, Статистическая гидромеханика: механика турбулентности, vol. 2, Nauka, Moscow, 1965. Translated as Statistical fluid mechanics, Volume 2, Dover, Mineola, NY, 2007.

[Ostoja-Starzewski 2008] M. Ostoja-Starzewski, Microstructural randomness and scaling in mechanics of materials, Chapman \& Hall/CRC, Boca Raton, FL, 2008.

[Ostoja-Starzewski et al. 2013] M. Ostoja-Starzewski, L. Shen, and A. Malyarenko, "Tensor random fields in conductivity and classical or microcontinuum theories", Math. Mech. Solids (2013).

[Porcu et al. 2012] E. Porcu, J.-M. Montero, and M. Schlather, Challenges in space-time modelling of natural events, Springer, Berlin, 2012.

[Robertson 1940] H. P. Robertson, "The invariant theory of isotropic turbulence", Proc. Cambridge Philos. Soc. 36 (1940), 209-223.

[Sena et al. 2013] M. P. Sena, M. Ostoja-Starzewski, and L. Costa, "Stiffness tensor random fields through upscaling of planar random materials", Probab. Eng. Mech. (2013).

[Spencer 1971] A. J. M. Spencer, "Theory of invariants”, pp. 239-353 in Continuum physics, vol. 1, edited by A. C. Eringen, Academic Press, New York, 1971.

[Varshalovich et al. 1975] D. A. Varshalovich, A. N. Moskalev, and V. K. Khersonskiй, Квантовая теория углового момента: аппарат неприводимых тензоров, сферические функции, 3nj-симболы, Nauka, Leningrad, 1975. Translated as Quantum theory of angular momentum: irreducible tensors, spherical harmonics, vector coupling coefficients, 3 nj symbols, World Scientific, Teaneck, NJ, 1988.

[Yaglom 1957] A. M. Yaglom, "Some classes of random fields in $n$-dimensional space, related to stationary stochastic processes", Theor. Probab. Appl. 2:3 (1957), 273-320.

Received 12 Feb 2013. Revised 15 Jul 2013. Accepted 11 Sep 2013.

ANATOLIY MALYARENKO: anatoliy.malyarenko@mdh.se

Division of Applied Mathematics, Mälardalen University, Box 883, Högskoleplan 1,

SE-721 23 Västerås, Sweden

MARTIN OSTOJA-STARZEWSKI: martinos@illinois.edu

Department of Mechanical Science and Engineering, University of Illinois at Urbana-Champaign, 1206 W. Green Street, Urbana, IL 61801-2906, United States 



\section{Guidelines for Authors}

Authors may submit manuscripts in PDF format on-line at the submission page.

Originality. Submission of a manuscript acknowledges that the manuscript is original and and is not, in whole or in part, published or under consideration for publication elsewhere. It is understood also that the manuscript will not be submitted elsewhere while under consideration for publication in this journal.

Language. Articles in MEMOCS are usually in English, but articles written in other languages are welcome.

Required items. A brief abstract of about 150 words or less must be included. It should be selfcontained and not make any reference to the bibliography. If the article is not in English, two versions of the abstract must be included, one in the language of the article and one in English. Also required are keywords and a Mathematics Subject Classification or a Physics and Astronomy Classification Scheme code for the article, and, for each author, postal address, affiliation (if appropriate), and email address if available. A home-page URL is optional.

Format. Authors are encouraged to use $\mathrm{IAT}_{\mathrm{E} X}$ and the standard amsart class, but submissions in other varieties of $\mathrm{T}_{\mathrm{E}} \mathrm{X}$, and exceptionally in other formats, are acceptable. Initial uploads should normally be in PDF format; after the refereeing process we will ask you to submit all source material.

References. Bibliographical references should be complete, including article titles and page ranges. All references in the bibliography should be cited in the text. The use of $\mathrm{BIBT}_{\mathrm{E}} \mathrm{X}$ is preferred but not required. Tags will be converted to the house format, however, for submission you may use the format of your choice. Links will be provided to all literature with known web locations and authors are encouraged to provide their own links in addition to those supplied in the editorial process.

Figures. Figures must be of publication quality. After acceptance, you will need to submit the original source files in vector graphics format for all diagrams in your manuscript: vector EPS or vector PDF files are the most useful.

Most drawing and graphing packages - Mathematica, Adobe Illustrator, Corel Draw, MATLAB, etc. - allow the user to save files in one of these formats. Make sure that what you are saving is vector graphics and not a bitmap. If you need help, please write to graphics@msp.org with as many details as you can about how your graphics were generated.

Bundle your figure files into a single archive (using zip, tar, rar or other format of your choice) and upload on the link you been provided at acceptance time. Each figure should be captioned and numbered so that it can float. Small figures occupying no more than three lines of vertical space can be kept in the text ("the curve looks like this:"). It is acceptable to submit a manuscript with all figures at the end, if their placement is specified in the text by means of comments such as "Place Figure 1 here". The same considerations apply to tables.

White Space. Forced line breaks or page breaks should not be inserted in the document. There is no point in your trying to optimize line and page breaks in the original manuscript. The manuscript will be reformatted to use the journal's preferred fonts and layout.

Proofs. Page proofs will be made available to authors (or to the designated corresponding author) at a Web site in PDF format. Failure to acknowledge the receipt of proofs or to return corrections within the requested deadline may cause publication to be postponed. 
Mathematics and Mechanics of Complex Systems vol. 2 no. 2

A mixed boundary value problem in potential theory for a bimaterial porous region: An application in the environmental geosciences

A. P. S. Selvadurai

Geometric degree of nonconservativity

Jean Lerbet, Marwa Aldowaji, Noël Challamel, Oleg N.

Kirillov, François Nicot and Félix Darve

Asymptotic analysis of small defects near a singular point in antiplane elasticity, with an application to the nucleation of a crack at a notch

Thi Bach Tuyet Dang, Laurence Halpern and Jean-Jacques Marigo

The homogenized behavior of unidirectional fiber-reinforced composite materials in the case of debonded fibers

Yahya Berrehili and Jean-Jacques Marigo

Statistically isotropic tensor random fields: Correlation structures

Anatoliy Malyarenko and Martin Ostoja-Starzewski

MEMOCS is a journal of the International Research Center for the Mathematics and Mechanics of Complex Systems at the Università dell' Aquila, Italy.

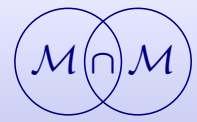

